\title{
S3-Leitlinie Kolorektales Karzinom Version 1.0 - Juni 2013 AWMF-Registernummer: 021/0070L
}

\section{S3-Guideline Colorectal Cancer Version 1.0}

Autoren

Institute
C. Pox ${ }^{1}$, S. Aretz ${ }^{2}$, S. C. Bischoff ${ }^{3}$, U. Graeven ${ }^{4}$, M. Hass ${ }^{5}$, P. Heußner ${ }^{6}$, W. Hohenberger ${ }^{7}$, A. Holstege ${ }^{8}$, J. Hübner ${ }^{9}$

F. Kolligs ${ }^{10}$, M. Kreis ${ }^{11}$, P. Lux ${ }^{7}$, J. Ockenga ${ }^{12}$, R. Porschen ${ }^{13}$, S. Post ${ }^{14}$, N. Rahner ${ }^{15}$, A. Reinacher-Schick ${ }^{16}$, J. F. Riemann ${ }^{17}$, R. Sauer ${ }^{18}$, A. Sieg ${ }^{19}$, W. Scheppach ${ }^{20}$, W. Schmitt ${ }^{21}$, H. J. Schmoll ${ }^{22}$, K. Schulmann ${ }^{23}$, A. Tannapfel ${ }^{24}$, W. Schmiegel ${ }^{1}$

Die Institutsangaben sind am Ende des Beitrags gelistet.
Bibliografie

DOI http://dx.doi.org/ 10.1055/s-0033-1350264

Z Gastroenterol 2013; 51: 753-854 @ Georg Thieme Verlag KG Stuttgart - New York . ISSN 0044-2771

Korrespondenzadresse Prof. Dr. Wolff Schmiegel Medizinische Klinik, RuhrUniversität Bochum, Knappschaftskrankenhaus In der Schornau 23 - 25 44892 Bochum Tel. :+ 49/2 34/2993401 Fax: ++ 49/2 34/2993409 meduni-kkh@rub.de www.medunikkh.de

\begin{tabular}{|c|c|c|}
\hline \multicolumn{2}{|c|}{ Inhaltsverzeichnis } & \multirow{2}{*}{$\begin{array}{l}\text { Seite } \\
755\end{array}$} \\
\hline 1. & Informationen zu dieser Leitlinie & \\
\hline 1.1. & Herausgeber & 755 \\
\hline 1.2. & Federführende Fachgesellschaft & 755 \\
\hline 1.3. & Finanzierung der Leitlinie & 755 \\
\hline 1.4. & Kontakt & 755 \\
\hline 1.5. & Zitierweise & 755 \\
\hline 1.6. & Besonderer Hinweis & 755 \\
\hline 1.7. & $\begin{array}{l}\text { Ziele des Leitlinienprogramms } \\
\text { Onkologie }\end{array}$ & 756 \\
\hline 1.8. & Autoren dieser Leitlinie & 756 \\
\hline 1.8 .1 & $\begin{array}{l}\text { Zusammensetzung der Leitlinien- } \\
\text { gruppe } 2011 / 2012\end{array}$ & 756 \\
\hline 1.8 .2 & $\begin{array}{l}\text { Koordinatoren der Leitliniengruppe } \\
2007 / 2008\end{array}$ & 756 \\
\hline 1.8.3. & $\begin{array}{l}\text { Zusammensetzung der AG- } \\
\text { Qualitätsindikatoren }\end{array}$ & 757 \\
\hline 1.8 .4 & Methodische Begleitung & 757 \\
\hline 1.9. & $\begin{array}{l}\text { Verfügbare Dokumente zur Leitlinie } \\
\text { und Implementierung }\end{array}$ & 757 \\
\hline 2. & Einführung & 758 \\
\hline 2.1. & Geltungsbereich und Zweck & 758 \\
\hline 2.1.1. & Zielsetzung und Fragestellung & 758 \\
\hline 2.1 .2 & Adressaten & 758 \\
\hline 2.1.3. & $\begin{array}{l}\text { Gültigkeitsdauer und Aktualisie- } \\
\text { rungsverfahren }\end{array}$ & 758 \\
\hline 2.2. & Grundlagen der Methodik & 758 \\
\hline 2.2.1. & $\begin{array}{l}\text { Schema der Evidenzgraduierung nach } \\
\text { Oxford }\end{array}$ & 758 \\
\hline 2.2 .2 & Schema der Empfehlungsgraduierung & 758 \\
\hline 2.2.3. & $\begin{array}{l}\text { Klinische Beurteilung - Klassifizierung } \\
\text { der Empfehlungsgrade }\end{array}$ & 759 \\
\hline 2.2.4. & Klassifikation der Konsensusstärke & 759 \\
\hline 2.2.5. & Statements & 759 \\
\hline 2.2 .6 & Good Clinical Practice (GCP) & 760 \\
\hline 2.2.7. & $\begin{array}{l}\text { Unabhängigkeit und Darlegung } \\
\text { möglicher Interessenkonflikte }\end{array}$ & 760 \\
\hline 2.3. & Verwendete Abkürzungen & 760 \\
\hline 2.4. & Redaktioneller Hinweis: & 761 \\
\hline 3. & $\begin{array}{l}\text { Prävention asymptomatische } \\
\text { Bevölkerung }\end{array}$ & 761 \\
\hline 3.1. & Lebensgewohnheiten & 761 \\
\hline 3.2. & Ernährungsempfehlungen & 761 \\
\hline 3.3. & Mikronährstoffe & 763 \\
\hline 3.4. & Medikamente & 763 \\
\hline
\end{tabular}

\begin{tabular}{|c|c|c|}
\hline \multicolumn{2}{|c|}{ Inhaltsverzeichnis } & \multirow{2}{*}{$\begin{array}{l}\text { Seite } \\
764\end{array}$} \\
\hline 4. & $\begin{array}{l}\text { Vorsorge/Früherkennung asympto- } \\
\text { matische Bevölkerung }\end{array}$ & \\
\hline 4.1. & Vorsorge-/Früherkennungs-Alter & 764 \\
\hline 4.2. & $\begin{array}{l}\text { Untersuchungsverfahren für die } \\
\text { Darmkrebsfrüherkennung/-vorsorge }\end{array}$ & 764 \\
\hline 4.2.1. & Endoskopische Verfahren & 764 \\
\hline 4.2.1.1. & Koloskopie & 764 \\
\hline 4.2.1.2. & Sigmoidoskopie & 765 \\
\hline 4.2.1.3. & Kapsel-Koloskopie & 766 \\
\hline 4.2.2. & Stuhltests & 766 \\
\hline 4.2.2.1. & Fäkaler okkulter Bluttest (FOBT) & 766 \\
\hline 4.2.2.2. & Genetische Stuhltests & 767 \\
\hline 4.2.2.3. & M2-PK & 767 \\
\hline 4.2 .3 & Radiologische Verfahren & 767 \\
\hline 4.3. & Kosteneffektivität & 768 \\
\hline 4.4 & $\begin{array}{l}\text { Empfehlungen anderer eingeschlos- } \\
\text { sener Leitlinien }\end{array}$ & 768 \\
\hline 5. & Risikogruppen & 768 \\
\hline 5.1 & Sporadisches kolorektales Karzinom & 768 \\
\hline 5.1.1. & Risikogruppen & 768 \\
\hline 5.1 .1 .1 & $\begin{array}{l}\text { Verwandte von Patienten mit kolo- } \\
\text { rektalem Karzinom }\end{array}$ & 768 \\
\hline 5.1 .1 .2 & $\begin{array}{l}\text { Verwandte von Patienten mit kolo- } \\
\text { rektalem Adenom }\end{array}$ & 769 \\
\hline 5.1.1.3. & $\begin{array}{l}\text { Patienten mit kolorektalen } \\
\text { Adenomen }\end{array}$ & 769 \\
\hline 5.1 .2 & Primärprävention & 769 \\
\hline 5.1.3. & Vorsorgeuntersuchungen & 769 \\
\hline 5.1.3.1. & $\begin{array}{l}\text { Verwandte ersten Grades von Patien- } \\
\text { ten mit kolorektalem Karzinom }\end{array}$ & 769 \\
\hline 5.1.3.2. & $\begin{array}{l}\text { Verwandte von Patienten mit kolo- } \\
\text { rektalen Adenomen }\end{array}$ & 770 \\
\hline 5.2 & Hereditäre kolorektale Karzinome & 770 \\
\hline 5.2.1. & Risikogruppen & 771 \\
\hline 5.2.1.1. & $\begin{array}{l}\text { HNPCC (hereditäres kolorektales } \\
\text { Karzinom ohne Polyposis)/Lynch- } \\
\text { Syndrom }\end{array}$ & 771 \\
\hline 5.2.1.2. & Adenomatöse Polyposis-Syndrome & 772 \\
\hline 5.2.1.2.1. & $\begin{array}{l}\text { Patienten mit klassischer familiärer } \\
\text { adenomatöser Polyposis (FAP) }\end{array}$ & 772 \\
\hline 5.2.1.2.2. & $\begin{array}{l}\text { Patienten mit attenuierter familiärer } \\
\text { adenomatöser Polyposis (AFAP) }\end{array}$ & 772 \\
\hline 5.2.1.2.3. & $\begin{array}{l}\text { Patienten mit MUTYH-assoziierter } \\
\text { Polyposis (MAP) }\end{array}$ & 773 \\
\hline
\end{tabular}


5.2.2. Vorsorgeuntersuchungen 773

5.2.2.1. HNPCC/Lynch-Syndrom 773

5.2.2.2. Adenomatöse Polyposis-Syndrome 775

5.2.2.2.1. Patienten mit klassischer familiärer adenomatöser 775 Polyposis (FAP)

5.2.2.2.2. Patienten mit attenuierter familiärer adenomatöser 777 Polyposis

5.2.2.2.3. Patienten mit MUTYH-assoziierter Polyposis (MAP) 778

5.2.2.3. Nicht adenomatöse Polyposis-Syndrome 779

5.3. Chronisch entzündliche Darmerkrankungen 780

5.3.1. Colitis ulcerosa $\quad 780$

5.3.2. Morbus Crohn 781

5.4. Anhang: Abbildungen und Tabellen des TK III 781

5.4.1. Algorithmus: Genetische Diagnostik und Vorsorge 781

5.4.2. Testalgorithmus Immunhistochemie/MSI zur 782 Abklärung Mismatch-Reparatur-Defekt

5.4.3. Empfohlenes Krebsfrüherkennungsprogramm 782 bei HNPCC

5.4.4. Spigelman-Klassifikation 782

6. Endoskopie: Durchführung und Polypenmanagement 782

6.1. Stellenwert der Endoskopie in der Diagnostik 782

$\begin{array}{lll} & \text { von Polypen und kolorektalen Karzinomen } \\ \text { 6.1.1. Sigmoidoskopie versus Koloskopie } & 783\end{array}$

6.1.2. Chromoendoskopie 783

6.1.3. Zoomendoskopie 783

6.2. Polypektomiedurchführung 784

6.2.1. Schlingenektomie versus Zangenektomie 784

6.3. Histologische Untersuchung 785

6.4. Vorgehen bei pT1-Karzinomen 785

6.5. Polypenmanagement (Nachsorge) 786

6.6. Medikamentöse Sekundärprävention bei Adenomen 787

7. Präoperative Diagnostik und Chirurgie 787

7.1. Einleitung $\quad 787$

7.2. Definition von Kolon- und Rektumkarzinomen 787

7.3. Definition Interdisziplinäre Tumorkonferenz $\quad 787$

$\begin{array}{lll}\text { 7.4. Präoperative Ausbreitungsdiagnostik } & 788\end{array}$

7.4.1. Endoskopische Diagnostik 788

7.4.2. Bildgebende Verfahren 788

7.4.2.1. Bedeutung einzelner bildgebender Verfahren (außer 788 PET) zur Abklärung von Fernmetastasen bei der Primärbehandlung des kolorektalen Karzinoms

7.4.2.2. Stellenwert des präoperativen lokalen Stagings durch 789 CT (MRT) beim Kolonkarzinom bez. lokaler Ausbreitung

7.4.2.3. Stellenwert der PET-CT

7.4.2.3.1. bei der Primärdiagnostik des kolorektalen Karzinoms 789

7.4.2.3.2. vor Resektion kolorektaler Lebermetastasen 789

7.4.3. Tumormarker $\quad 790$

7.4.4. Spezielle Diagnostik beim Rektumkarzinom 790

7.5. Chirurgische Therapie mit kurativem Ziel 791

7.5.1. Intraoperatives Staging 791

7.5.1.1. Intraoperative pathologische Diagnostik 792

7.5.2. Radikalchirurgische Therapie des Kolonkarzinoms 792

7.5.2.1. Ausmaß der Lymphknotendissektion: 792

7.5.2.2. Komplette Mesokolische Exzision (CME) 793

7.5.2.3. Lokalablative Verfahren bei Lebermetastasen 793

7.5.2.3.1. Radiofrequenzablation (RFA) 793

7.5.2.3.2. Selective Internal Radiation Therapy (SIRT) 793

7.5.2.3.3. Laserinduzierte interstitielle Thermotherapie (LITT) 794

7.5.3. Radikalchirurgische Therapie des Rektumkarzinoms 794

7.5.3.1. Allgemeine onkologische Grundsätze 794

7.5.3.2. Vorgehen bei Tumoren des oberen Rektumdrittels 794

7.5.3.3. Vorgehen bei Tumoren des mittleren und unteren 794 Rektumdrittels

7.5.3.4. Rekonstruktion nach total mesorektaler Exzision 795
Inhaltsverzeichnis

Seite

7.5.3.5. Entscheidung zum Sphinkter-Erhalt 795

7.5.3.6. Vorgehen bei komplettem Response nach 795

7.5.3.7. Rektum-Exstirpation 796

7.5.4. Stoma-Anlage 796

7.5.5. Lokale Operationsverfahren des Rektumkarzinoms 796

7.6. Laparoskopische Chirurgie 797

7.7. Sondersituationen 797

7.7.1. Chirurgische Therapie der Peritonealkarzinose 797

7.7.2. Multiviszerale Resektion 797

7.7.3. Mehrfachkarzinome des Kolorektums 798

7.7.4. Notfalloperationen 798

7.7.5. Resektion von Lebermetastasen 798

7.7.6. Ausgedehnte Fernmetastasierung und asympto- $\quad 798$

7.7.7. Karzinome auf dem Boden eines hereditären kolo- 798 rektalen Karzinoms ohne Polyposis (HNPCC)

7.7.8. Karzinome auf dem Boden einer familiären adenoma- 799 tösen Polyposis (FAP)

7.7.9. Karzinome auf dem Boden einer Colitis ulcerosa 799

7.8. Postoperative pathohistologische Diagnostik 799

7.8.1. Karzinom-Graduierung in Abhängigkeit von MSI-H 799

7.8.2. Anzahl zu entfernender LK 800

7.8.3. Bedeutung des Tumorabstands zur Resektionsfläche 800 des Mesokolons beim Kolonkarzinom

7.8.4. Bedeutung des Abstands vom zirkumferenziellen 800 Resektionsrand (CRM-Klassifikation) beim Kolon- und Rektumkarzinom

7.8.5. Bedeutung der Angabe der Qualität des TME-Präparats 801

7.9. Psychoonkologische Aspekte 801

8. Adjuvante und neoadjuvante Therapie 802

8.1. Adjuvante Therapie des Kolonkarzinoms 802

8.1.1. Indikation zur adjuvanten Behandlung bei 802 Kolonkarzinomen

8.1.2. Altersbeschränkung für die Durchführung einer 802

8.1.3. UICC-Stadium III 802

8.1.4. UICC-Stadium II 803

8.1.5. UICC-Stadium II mit Risikofaktoren 803

8.1.6. Chemotherapieprotokolle 804

8.1.6.1. Stadium III $\quad 804$

8.1.6.2. Stadium II 805

8.2. Perioperative Therapie beim Rektumkarzinom 805

8.2.1. Indikationen zur perioperativen Radio- oder 805

$\begin{array}{lll} & \text { Radiochemotherapie } & \\ \text { 8.2.1.1. Stadium I } & 805\end{array}$

8.2.1.2. Stadium II/III 806

8.2.2. Adjuvante Therapie 807

8.2.2.1. Adjuvante Therapie bei primärer Operation (ohne 807 neoadjuvante Therapie)

8.2.2.2. Adjuvante Therapie nach neoadjuvanter Radiotherapie 808 oder Radiochemotherapie

9. Therapeutisches Vorgehen bei Metastasierung und in 809 der palliativen Situation

9.1. Primär resektable Leber und/oder Lungenmetastasen 809

9.1.1. Primär resektable Lungenmetastasen 809

9.1.2. Primär resektable Lebermetastasen 809

9.1.2.1. Präoperative Bildgebung 810

9.1.2.2. Perioperative Therapie primär resektabler Leber- 811 metastasen

9.1.2.2.1. Neoadjuvante Therapie resektabler Lebermetastasen 811

9.1.2.2.2. Adjuvante Therapie resektabler Lebermetastasen 812

9.2. Patienten mit einer Indikation für eine intensivierte 813 systemische Therapie

9.2.1. Patienten mit potenziell resektablen Metastasen 813 


\begin{tabular}{|c|c|c|}
\hline \multicolumn{2}{|c|}{ Inhaltsverzeichnis } & \multirow{2}{*}{$\begin{array}{l}\text { Seite } \\
814\end{array}$} \\
\hline 9.2.1.1. & $\begin{array}{l}\text { Vorgehen bei isolierten primär irresektablen } \\
\text { Lungenmetastasen }\end{array}$ & \\
\hline 9.2.1.2. & $\begin{array}{l}\text { Vorgehen bei isolierten primär irresektablen } \\
\text { Lebermetastasen }\end{array}$ & 814 \\
\hline 9.2.1.2.1. & Systemische neoadjuvante Therapie & 814 \\
\hline 9.2.1.2.2. & $\begin{array}{l}\text { Chemotherapiefolgen auf das gesunde Lebergewebe } \\
\text { und Metastasenlokalisation }\end{array}$ & 814 \\
\hline 9.2.1.2.3. & Lokoregionäre Therapieverfahren & 815 \\
\hline 9.2.2. & $\begin{array}{l}\text { Patienten mit einer Indikation für eine intensivierte } \\
\text { palliative Therapie }\end{array}$ & 815 \\
\hline 9.3 . & $\begin{array}{l}\text { Patienten mit der Möglichkeit für eine weniger } \\
\text { intensive Therapie }\end{array}$ & 815 \\
\hline 9.4 . & Chemotherapieprotokolle & 816 \\
\hline 9.4.1. & Erstlinientherapie & 816 \\
\hline 9.4.1.1. & Monotherapie (5-FU) & 816 \\
\hline 9.4.1.2. & Kombinationstherapie & 817 \\
\hline 9.4 .2 & $\begin{array}{l}\text { Therapiedauer/Therapiepause in der Erstlinien- } \\
\text { therapie-Reinduktion }\end{array}$ & 821 \\
\hline 9.4.3. & $\begin{array}{l}\text { Chemotherapieprotokolle in der Zweit- und } \\
\text { Drittlinientherapie }\end{array}$ & 821 \\
\hline 9.4.3.1. & $\begin{array}{l}\text { Kombinationstherapie in der Zweit- und Dritt- } \\
\text { linientherapie }\end{array}$ & 822 \\
\hline 9.4.3.2. & $\begin{array}{l}\text { Monotherapie mit biologischen Substanzen in der } \\
\text { Drittlinienlinientherapie }\end{array}$ & 823 \\
\hline 9.5 & $\begin{array}{l}\text { Vorgehen beim Lokalrezidiv oder bei nicht hepatischer } \\
\text { und nicht pulmonaler Fernmetastasierung }\end{array}$ & 823 \\
\hline 9.5.1. & Lokalrezidiv & 823 \\
\hline 9.5.2. & $\begin{array}{l}\text { Nicht hepatische oder nicht pulmonale Fern- } \\
\text { metastasen }\end{array}$ & 824 \\
\hline 10. & Nachsorge & 824 \\
\hline 10.1. & Nachsorge bei Patienten im UICC-Stadium I & 824 \\
\hline 10.2 & Nachsorge bei Patienten im UICC-Stadium II und III & 825 \\
\hline 10.3. & Wertigkeit diagnostischer Methoden in der Nachsorge & 825 \\
\hline 10.3.1. & Anamneseerhebung & 825 \\
\hline 10.3.2. & CEA Bestimmung & 825 \\
\hline 10.3.3. & Weitere Laborwerte & 825 \\
\hline 10.3.4. & FOBT & 826 \\
\hline 10.3.5. & Sonografie & 826 \\
\hline 10.3.6. & Endosonografie & 826 \\
\hline 10.3.7. & Röntgen-Thorax-Untersuchung & 826 \\
\hline 10.3.8. & Computertomografie & 826 \\
\hline 10.3.9. & Endoskopische Verfahren & 827 \\
\hline 10.3.9.1. & Koloskopie & 827 \\
\hline 10.3.9.2. & Sigmoidoskopie & 827 \\
\hline 10.3.9.3. & Rektoskopie & 827 \\
\hline 10.3.10. & Kolonkontrasteinlauf, virtuelle Kolonografie und PET & 828 \\
\hline 10.4 & Zeitlicher Ablauf der Nachsorge & 828 \\
\hline 10.5 & Altersbegrenzung der Nachsorge & 828 \\
\hline 10.6. & Sonderfälle & 828 \\
\hline 10.7 & $\begin{array}{l}\text { Rehabilitation nach Resektion eines kolorektalen } \\
\text { Karzinoms }\end{array}$ & 829 \\
\hline 10.8. & Tertiärprävention & 829 \\
\hline 10.8.1. & $\begin{array}{l}\text { Versorgungskontinuität und Fortführung der gesund- } \\
\text { heitsförderlichen Aktivitäten nach Akuttherapie und } \\
\text { Rehabilitation }\end{array}$ & 829 \\
\hline 10.8.1.1. & Verbesserung der Versorgungskontinuität & 829 \\
\hline 10.8.1.2. & Fortführung der gesundheitsförderlichen Aktivitäten & 829 \\
\hline 10.8 .2 & $\begin{array}{l}\text { Tertiärprävention: Physikalische Aktivität, Ernährung } \\
\text { sowie komplementäre und alternative Therapie }\end{array}$ & 830 \\
\hline 10.8.2.1. & Physische Aktivität & 830 \\
\hline 10.8.2.2. & Ernährung & 830 \\
\hline 10.8.2.3. & Komplementäre und alternative Medizin & 830 \\
\hline 10.8.2.4. & Alternative Heilmethoden & 830 \\
\hline 11. & Qualitätsindikatoren & 830 \\
\hline 12. & Anhang & 833 \\
\hline
\end{tabular}

\begin{tabular}{|lll|}
\hline \multicolumn{2}{l}{ Inhaltsverzeichnis } & Seite \\
\hline 12.1. & UICC-Klassifikation des kolorektalen Karzinoms & 833 \\
\hline 12.2. & Amsterdam-Kriterien & 833 \\
\hline 12.3. & Revidierte Bethesda-Kriterien & 833 \\
\hline 13. & Abbildungsverzeichnis & 833 \\
\hline 14. & Tabellenverzeichnis & 833 \\
\hline 15. & Literatur & 834 \\
\hline
\end{tabular}

\section{Informationen zu dieser Leitlinie}

$\nabla$

\subsection{Herausgeber}

Leitlinienprogramm Onkologie der AWMF, Deutschen Krebsgesellschaft e.V. und Deutschen Krebshilfe e.V.

\subsection{Federführende Fachgesellschaft}

Deutsche Gesellschaft für Verdauungs- und Stoffwechselkrankheiten (DGVS)

Steuerungsgruppe für Planung und Durchführung über DGVS: Univ.-Prof. Dr. Wolff Schmiegel

Dr. Christian P. Pox

\subsection{Finanzierung der Leitlinie}

Diese Leitlinie wurde von der Deutschen Krebshilfe im Rahmen des Onkologischen Leitlinienprogramms gefördert.

\subsection{Kontakt}

Leitlinienprogramm Onkologie Office

c/o Deutsche Krebsgesellschaft e.V.

Kuno-Fischer-Str. 8

14057 Berlin

leitlinienprogramm@krebsgesellschaft.de

www.leitlinienprogramm-onkologie.de

\subsection{Zitierweise}

Leitlinienprogramm Onkologie (Deutsche Krebsgesellschaft, Deutsche Krebshilfe, AWMF): S3-Leitlinie Kolorektales Karzinom, Langversion 1.0, AWMF Registernummer: 021 -007OL, http:// leitlinienprogramm-onkologie.de/Leitlinien.7.0.html

\subsection{Besonderer Hinweis}

Die Medizin unterliegt einem fortwährenden Entwicklungsprozess, sodass alle Angaben, insbesondere zu diagnostischen und therapeutischen Verfahren, immer nur dem Wissensstand zurzeit der Drucklegung der Leitlinie entsprechen können. Hinsichtlich der angegebenen Empfehlungen zur Therapie und der Auswahl sowie Dosierung von Medikamenten wurde die größtmögliche Sorgfalt beachtet. Gleichwohl werden die Benutzer aufgefordert, die Beipackzettel und Fachinformationen der Hersteller zur Kontrolle heranzuziehen und im Zweifelsfall einen Spezialisten zu konsultieren. Fragliche Unstimmigkeiten sollen bitte im allgemeinen Interesse der OL-Redaktion mitgeteilt werden.

Der Benutzer selbst bleibt verantwortlich für jede diagnostische und therapeutische Applikation, Medikation und Dosierung.

In dieser Leitlinie sind eingetragene Warenzeichen (geschützte Warennamen) nicht besonders kenntlich gemacht. Es kann also aus dem Fehlen eines entsprechenden Hinweises nicht geschlossen werden, dass es sich um einen freien Warennamen handelt.

Das Werk ist in allen seinen Teilen urheberrechtlich geschützt. Jede Verwertung außerhalb der Bestimmung des Urhebergeset- 
zes ist ohne schriftliche Zustimmung des Leitlinienprogramms Onkologie (OL) unzulässig und strafbar. Kein Teil des Werkes darf in irgendeiner Form ohne schriftliche Genehmigung des OL reproduziert werden. Dies gilt insbesondere für Vervielfältigungen, Übersetzungen, Mikroverfilmungen und die Einspeicherung, Nutzung und Verwertung in elektronischen Systemen, Intranets und dem Internet.

\subsection{Ziele des Leitlinienprogramms Onkologie}

Die Arbeitsgemeinschaft der Wissenschaftlichen Medizinischen Fachgesellschaften e.V., die Deutsche Krebsgesellschaft e.V. und die Deutsche Krebshilfe e.V. haben sich mit dem Leitlinienprogramm Onkologie (OL) das Ziel gesetzt, gemeinsam die Entwicklung und Fortschreibung und den Einsatz wissenschaftlich begründeter und praktikabler Leitlinien in der Onkologie zu fördern und zu unterstützen. Die Basis dieses Programms beruht auf den medizinisch-wissenschaftlichen Erkenntnissen der Fachgesellschaften und der DKG, dem Konsens der medizinischen Fachexperten, Anwender und Patienten sowie auf dem Regelwerk für die Leitlinienerstellung der AWMF und der fachlichen Unterstützung und Finanzierung durch die Deutsche Krebshilfe. Um den aktuellen Stand des medizinischen Wissens abzubilden und den medizinischen Fortschritt zu berücksichtigen, müssen Leitlinien regelmäßig überprüft und fortgeschrieben werden. Die Anwendung des AWMF-Regelwerks soll hierbei Grundlage zur Entwicklung qualitativ hochwertiger onkologischer Leitlinien sein. Da Leitlinien ein wichtiges Instrument der Qualitätssicherung und des Qualitätsmanagements in der Onkologie darstellen, sollten sie gezielt und nachhaltig in den Versorgungsalltag eingebracht werden. So sind aktive Implementierungsmaßnahmen und auch Evaluationsprogramme ein wichtiger Bestandteil der Förderung des Leitlinienprogramms Onkologie. Ziel des Programms ist es, in Deutschland professionelle und mittelfristig finanziell gesicherte Voraussetzungen für die Entwicklung und Bereitstellung hochwertiger Leitlinien zu schaffen. Denn diese hochwertigen Leitlinien dienen nicht nur dem strukturierten Wissenstransfer, sondern können auch in der Gestaltung der Strukturen des Gesundheitssystems ihren Platz finden. Zu erwähnen sind hier evidenzbasierte Leitlinien als Grundlage zum Erstellen und Aktualisieren von Disease Management Programmen oder die Verwendung von aus Leitlinien extrahierten Qualitätsindikatoren im Rahmen der Zertifizierung von Organtumorzentren.

\subsection{Autoren dieser Leitlinie}

C. Pox, S. Aretz, S.C. Bischoff, U. Graeven, M. Hass, P. Heußner, W. Hohenberger, A. Holstege, J. Hübner, F. Kolligs, M. Kreis, P. Lux, J. Ockenga, R. Porschen, S. Post, N. Rahner, A. ReinacherSchick, J. F. Riemann, R. Sauer, A. Sieg, W. Scheppach, W. Schmitt, H.-J. Schmoll, K. Schulmann, A. Tannapfel, W. Schmiegel

1.8.1. Zusammensetzung der Leitliniengruppe $2011 / 2012$ Die Fachexpertengruppe für die Aktualisierung der Kapitel 3, 4, 5 , 7, 10 (s. Abschnitt 2.2.1) setzte sich aus insgesamt 53 Mandatsträgern und Plenumsmitgliedern folgender Fachgesellschaften, Berufsverbände und Patientenvereinigungen zusammen:
- Fachgesellschaften

DKG (Deutsche Krebsgesellschaft)

- ASORS (AG der DKG „Supportive Maßnahmen in der Onkologie, Rehabilitation und Sozialmedizin“)

- PRIO (AG der DKG Prävention und integrative Medizin in der Onkologie)

- PSO (AG der DKG: Dt. Arbeitsgemeinschaft für Psychoonkologie)

- кOK (AG der DKG: Konferenz Onkologische Kranken- und Kinderkrankenpflege)

- DGVS (Deutsche Gesellschaft für Verdauungs- und Stoffwechselkrankheiten)

DEGAM (Deutsche Gesellschaft für Allgemeinmedizin und Familienmedizin)

- DEGRO (Deutsche Gesellschaft für Radioonkologie)

- DGAV (Deutsche Gesellschaft für Allgemein- und Viszeralchirurgie)

- CACP (Chirurgische Arbeitsgemeinschaft für Colo-Proktologie)

- CAMIC (Chirurgische Arbeitsgemeinschaft für Minimalinvasive Chirurgie)

- CAO-V (Chirurgische Arbeitsgemeinschaft für Onkologie)

DGCH (Deutsche Gesellschaft für Chirurgie)

- DGEM (Deutsche Gesellschaft für Ernährungsmedizin)

- DGHO (Deutsche Gesellschaft für Hämatologie und Onkologie)

- DGIM (Deutsche Gesellschaft für Innere Medizin)

> DGKL (Deutsche Gesellschaft für Klinische Chemie und Laboratoriumsmedizin)

- DGN (Deutsche Gesellschaft für Nuklearmedizin)

D DGP (Deutsche Gesellschaft für Pathologie)

- DRG (Deutsche Röntgengesellschaft)

- GfH (Deutsche Gesellschaft für Humangenetik)

$\checkmark$ andere Institutionen

- bng (Berufsverband Niedergelassener Gastroenterologen Deutschlands e.V.)

- HÄV (Deutscher Hausärzteverband)

- AQUA (Institut für angewandte Qualitätsförderung und Forschung im Gesundheitswesen)

- ZI (Zentralinstitut der Kassenärztlichen Versorgung in der BRD)

Felix-Burda-Stiftung

- Stiftung Lebensblicke

- Patientenvereinigungen

- DCCV (Deutsche Morbus Crohn/Colitis Ulcerosa Vereinigung)

D Deutsche ILCO (Vereinigung für Stomaträger und für Menschen mit Darmkrebs)

- Tab. 1 zeigt die Zusammensetzung der Leitliniengruppe 2011/ 2012 unter der Leitung von Prof. Dr. W. Schmiegel (DKG, DGVS)

1.8.2. Koordinatoren der Leitliniengruppe $2007 / 2008$

Die Koordinatoren bei der Überarbeitung der Kapitel 6, 8 und 9 in 2007/2008 waren:

Kapitel 6 - Endoskopie: Durchführung und Polypenmanagement:

J.F. Riemann, W. Schmitt

Kapitel 8 - Adjuvante und neoadjuvante Therapie:

R. Porschen, R. Sauer

Kapitel 9 - Therapeutisches Vorgehen bei Metastasierung und in der palliativen Situation: U. Graeven, H-J. Schmoll 
Tab. 1 Zusammensetzung der Arbeitsgruppen für die Aktualisierung der Kapitel 3, 4, 5, 7, 10.

\begin{tabular}{|c|c|}
\hline \multicolumn{2}{|c|}{ Kapitel 3: Prävention asymptomatische Bevölkerung } \\
\hline Koordinator: J. F. Riemann & DGIM, DGVS, Stiftung Lebensblicke \\
\hline \multicolumn{2}{|l|}{ Mitglieder: } \\
\hline S. C. Bischoff & DGEM, DGVS \\
\hline F. Kolligs & DGVS \\
\hline J. Ockenga & DGEM, DGVS \\
\hline W. Scheppach & DGEM, DGVS \\
\hline \multicolumn{2}{|c|}{ Kapitel 4: Früherkennung/Vorsorge asymptomatische Bevölkerung } \\
\hline Koordinatoren: C. Pox, A. Sieg & beide DGVS \\
\hline \multicolumn{2}{|l|}{ Mitglieder: } \\
\hline L. Altenhofen & ZI \\
\hline H-J. Brambs & DRG, DGAM \\
\hline H. Brenner & Experte (nicht stimmberechtigt) \\
\hline P. Engeser & HÄV \\
\hline A. Theilmeier & bng, DGVS \\
\hline \multicolumn{2}{|l|}{ Kapitel 5: Risikogruppen } \\
\hline \multicolumn{2}{|l|}{ Koordinatoren: } \\
\hline N. Rahner & $\mathrm{GfH}$ \\
\hline K. Schulmann & DGVS \\
\hline G. Baretton & DGP \\
\hline B. Bokemeyer & bng, DGVS \\
\hline J. Epplen & DGHG \\
\hline U. Melle & DGVS \\
\hline R. Porschen & DGVS \\
\hline J. Weitz & DGAV \\
\hline C. Witte & DCCV \\
\hline \multicolumn{2}{|c|}{ Kapitel 7: Präoperative Diagnostik und Chirurgie } \\
\hline \multicolumn{2}{|l|}{ Koordinatoren: } \\
\hline W. Hohenberger & DKG, DGAV \\
\hline S. Post & DKG, DGAV, CACP \\
\hline M. Anthuber & DGAV \\
\hline W. Bechstein & DGAV \\
\hline U. Graeven & DGVS \\
\hline M. $\mathrm{Haß}$ & Dt. ILCO \\
\hline M. Heike & DGHO \\
\hline K.-W. Jauch & DGAV \\
\hline T. Kirchner & DGP \\
\hline H. Lang & DKG, DGAV, CAO-V \\
\hline K-H. Link & DKG, DGAV \\
\hline P. Pereira & DRG \\
\hline H-R. Raab & DGAV \\
\hline A. Reinacher-Schick & DGVS \\
\hline C. Rödel & DEGRO \\
\hline M. Sailer & DGAV \\
\hline R. Sauer & DEGRO \\
\hline K. Scheidhauer & DGN \\
\hline A. Tannapfel & DGP \\
\hline T. Vogl & DRG \\
\hline C. Wagener & DGKL \\
\hline M. Walz & DGAV, CAMIC \\
\hline C. Wittekind & DGP \\
\hline \multicolumn{2}{|l|}{ Kapitel 10: Nachsorge } \\
\hline Koordinator: A. Holstege & DGVS \\
\hline \multicolumn{2}{|l|}{ Mitglieder: } \\
\hline P. Heußner & PSO \\
\hline T. Höhler & DGHO \\
\hline J. Hübner & DKG, PRIO \\
\hline J. Körber & DKG, ASORS \\
\hline M. Landenberger & DKG, KOK \\
\hline H. Link & DKG, ASORS \\
\hline \multicolumn{2}{|c|}{ Zusätzlich beim Plenum beteiligt } \\
\hline S. Ludt & AQUA \\
\hline P. Lux & Mit-Autor (nicht stimmberechtigt) \\
\hline C. Maar & Felix-Burda-Stiftung \\
\hline
\end{tabular}

1.8.3. Zusammensetzung der AG Qualitätsindikatoren Die Entwicklung der Vorschläge zu Qualitätsindikatoren erfolgte durch die in $\odot$ Tab. 2 aufgeführte Arbeitsgruppe.

\subsubsection{Methodische Begleitung}

Durch das Leitlinienprogramm Onkologie:

- Prof. Dr. Ina Kopp (AWMF), Marburg

- Dr. Markus Follmann, MPH MSc (DKG), Berlin

\subsection{Verfügbare Dokumente zur Leitlinie und Implementierung}

Bei diesem Dokument handelt es sich um die Langversion der S3-Leitlinie Kolorektales Karzinom, welche über die folgenden Seiten zugänglich ist:

- AWMF (www.leitlinien.net)

- Leitlinienprogramm Onkologie (http://www.leitlinienpro gramm-onkologie.de/OL/leitlinien.html)

- Deutsche Krebsgesellschaft (http://www.krebsgesellschaft.de/ wub_llevidenzbasiert,120 884.html)

- Deutsche Krebshilfe (http://www.krebshilfe.de/)

- Guidelines International Network (www.g-i-n.net)

- Beteiligte Fachgesellschaften (z.B. http://www.dgvs.de/508. php)

Darüber hinaus wird die Langversion dieser Leitlinie wird in der Zeitschrift für Gastroenterologie veröffentlicht werden.

Neben der Langfassung wird es die folgenden ergänzenden Dokumente zu dieser Leitlinie geben:

- Kurzversion

- Leitlinienreport

- Übersetzung (englisch)

- Patientenleitlinie

- Externer Evidenzbericht

Alle diese Dokumente werden ebenfalls auf den oben genannten Homepages abrufbar sein.

Der externe Evidenzbericht wurde zum Kapitel 4 (Vorsorge/Früherkennung) und Teilen des Kapitels 7 (präoperative Diagnostik und Chirurgie) von einer Kooperation der Universität Witten/Herdecke (Institut für Forschung in der Operativen Medizin) mit der Universität Duisburg/Essen (Alfried Krupp von Bohlen und Halbach Stiftungslehrstuhl für Medizinmanagement) erstellt.

Tab. 2 Zusammensetzung der AG Qualitätsindikatoren.

\begin{tabular}{|c|c|}
\hline Name & Institution \\
\hline \multicolumn{2}{|l|}{ Koordinatoren: } \\
\hline W. Schmiegel & DKG, DGVS \\
\hline C. Pox & DGVS \\
\hline \multicolumn{2}{|l|}{ Mitglieder: } \\
\hline M. Haß & Dt. ILCO \\
\hline W. Hohenberger & DKG, DGAV \\
\hline A. Holstege & DGVS \\
\hline F. Kolligs & DGVS \\
\hline M. Klinkhammer-Schalke & $\begin{array}{l}\text { Arbeitsgemeinschaft Deutscher Tumorzentren } \\
\text { (ADT) }\end{array}$ \\
\hline I. Kopp & AWMF, Leitlinienprogramm Onkologie \\
\hline S. Ludt & AQUA \\
\hline J. Ockenga & DGVS \\
\hline S. Post & DKG, DGAV, CACP \\
\hline A. Tannapfel & DGP \\
\hline S. Wesselmann & $\begin{array}{l}\text { Deutsche Krebsgesellschaft (DKG), Bereich } \\
\text { Zertifizierung }\end{array}$ \\
\hline
\end{tabular}


Die Erstellung der Patientenleitlinie und die Übersetzung der Langversion ins Englische werden mit Fertigstellung der Leitlinie in Auftrag gegeben.

Eine flächendeckende Verbreitung dieser evidenzbasierten, aktuellen Verhaltensempfehlungen ist insbesondere für die Steigerung der Qualität bei Patienteninformation und -versorgung notwendig. Mithilfe standardisierter Methoden wurden von den Empfehlungen Qualitätsindikatoren abgeleitet. Diese dienen neben der Implementierung in erster Linie der Qualitätssicherung und -steigerung der patientenrelevanten Prozesse.

\section{Einführung}

$\nabla$

\subsection{Geltungsbereich und Zweck}

\subsubsection{Zielsetzung und Fragestellung}

Das kolorektale Karzinom (KRK) ist mit ca. 63000 Neuerkrankungen jedes Jahr (62 956 in 2010) und ca. 26000 Todesfällen im Jahr (25999 in 2010) in Deutschland einer der häufigsten malignen Tumoren (Quelle: Gesellschaft der epidemiologischen Krebsregister in Deutschland e.V. (GEKID)). 1999 wurde erstmalig von der DGVS in Zusammenarbeit mit der Deutschen Krebsgesellschaft eine S3-Leitlinie für das KRK veröffentlicht, die flächendeckend eine standardisiert hochwertige Patientenversorgung auf dem Boden evidenzbasierter Medizin erreichen sollte. Inzwischen ist auch eine europäische Leitlinie zum kolorektalen Karzinom veröffentlicht worden. Die Leitlinienkommission sah die Notwendigkeit einer Spezifizierung aufgrund der ungleichen Gesundheitssysteme und Versorgungsstandards in den europäischen Ländern. Diese hier vorliegende deutsche Leitlinie deckt gegenüber der europäischen Leitlinie weitere Teilbereiche ab und ist bestrebt, alle auf Deutschland bezogenen Fragestellungen zu berücksichtigen.

Die Leitlinie ist in acht Themenkomplexe (TK) gegliedert:

- TK I: Prävention asymptomatische Bevölkerung (s. Kapitel 3)

- TK II: Früherkennung/Vorsorge asymptomatische Bevölkerung (s. Kapitel 4)

- TK III: Risikogruppen (s. Kapitel 5)

- TKIV: Endoskopie: Durchführung und Polypenmanagement (s. Kapitel 6)

- TK V: Präoperative Diagnostik und Chirurgie (s. Kapitel 7)

- TK VI: Adjuvante und neoadjuvante Therapie (s. Kapitel 8)

- TK VII: Therapeutisches Vorgehen bei Metastasierung und in der palliativen Situation (s. Kapitel 9)

- TK VIII: Nachsorge (s. Kapitel 10)

Um die Empfehlungen auf dem neuesten Stand wissenschaftlicher Erkenntnisse zu halten, wird die Leitlinie seitdem in enger Zusammenarbeit mit der AWMF regelmäßig aktualisiert (2004 komplett und 2008 die Themenkomplexe IV, VI und VII). Die aktuelle Überarbeitung betrifft die Themenkomplexe I, II, III, V und VIII, sowie einzelne Abschnitte aus IV, VI und VII.

Im Aktualisierungsprozess 2012/2013 legte die Leitliniengruppe fest, dass u.a. zu folgenden Fragen Stellung genommen werden sollte:

- Welche Maßnahmen der Prävention können das KRK-Risiko reduzieren?

- Welche Methoden sollten zur Vorsorge/Früherkennung einsetzt werden?

- Welche Verfahren sollten in der präoperativen Diagnostik beim KRK eingesetzt werden?

- Was ist bei der Stomaanlage zu beachten?

- Wie ist der Stellenwert laparoskopischer Resektionsverfahren?
- Zu welchem Zeitpunkt beginnt die Tumornachsorge?

- Welchen Stellenwert haben Rehabilitation und Tertiärprävention nach kurativer Tumoroperation?

Entsprechend dieser Fragestellungen wurden alle Empfehlungen auf Aktualität überprüft und gegebenenfalls nach Literaturrecherchen überarbeitet.

In den Empfehlungskästen ist jeweils das Datum der letzten Überarbeitung (2008 oder 2013) aufgeführt.

\subsubsection{Adressaten}

Diese Leitlinie richtet sich vorrangig an:

- Ärztinnen und Ärzte, die in der Prävention und Behandlung des KRK im ambulanten und stationären Sektor tätig sind,

- Kooperationspartner der Ärzteschaft (Fachbereiche im Gesundheitswesen),

- Kostenträger.

\subsubsection{Gültigkeitsdauer und Aktualisierungsverfahrene}

Die Gültigkeitsdauer der 2011/2012 überarbeiteten Themenkomplexe I, II, III, V, und VIII bzw. Kapitel 3, 4, 5, 7, 10 wird auf 5 Jahre geschätzt, sie werden spätestens 2017 einer erneuten Revision unterzogen. Die Aktualisierung der verbleibenden Themenkomplexe IV, VI und VII bzw. Kapitel 6, 8, 9 ist für 2014 vorgesehen.

Das Aktualisierungsverfahren wird von der verantwortlichen Institution (Leitliniensekretariat) koordiniert. Neu erscheinende wissenschaftliche Erkenntnisse werden von den Mitgliedern der Leitliniengruppe beobachtet. Daraufhin werden einzelne Themenkomplexe gegebenenfalls vorzeitig überarbeitet. Alle Aktualisierungen werden gesondert publiziert (Addendum zur Internetversion, Fachzeitschriften) und anschließend in die Volltextversion der Leitlinie eingearbeitet.

Kommentare und Hinweise für den Aktualisierungsprozess aus der Praxis sind ausdrücklich erwünscht und können an das Leitliniensekretariat adressiert werden.

Leitlinienkoordination: Dr. Christian P. Pox

Leitliniensekretariat: Anjes Giuliani

Medizinische Klinik der Ruhr-Universität Bochum

Knappschaftskrankenhaus

In der Schornau $23-25$

44892 Bochum

Meduni-kkh@rub.de

\subsection{Grundlagen der Methodik}

Das methodische Vorgehen richtete sich nach dem AWMF Regelwerk (http://www.awmf-leitlinien.de) und ist im Leitlinienreport zu dieser Leitlinie (s. z. B. http://leitlinienprogramm-onkologie.de/Leitlinien.7.0.html) dargelegt.

\subsubsection{Schema der Evidenzgraduierung nach Oxford}

Zur Klassifikation des Verzerrungsrisikos der identifizierten Studien wurde in dieser Leitlinie das in $\bullet$ Tab. 3 aufgeführte System des Oxford Centre for Evidence-based Medicine in der Version von 2009 (verfügbar unter www.cebm.net) verwendet. Dieses System sieht die Klassifikation der Studien für verschiedene klinische Fragestellungen (Nutzen von Therapie, prognostische Aussagekraft, diagnostische Wertigkeit) vor

\subsubsection{Schema der Empfehlungsgraduierung}

Die OL-Methodik sieht eine Vergabe von Empfehlungsgraden durch die Leitlinien-Autoren im Rahmen eines formalen Konsensusverfahrens vor. Dementsprechend wurde ein durch die AWMF moderierter, mehrteiliger nominaler Gruppenprozess durchgeführt. Am 


\begin{tabular}{|c|c|c|c|c|c|}
\hline Level & $\begin{array}{l}\text { Therapy/Prevention, } \\
\text { Aetiology/Harm }\end{array}$ & Prognosis & Diagnosis & $\begin{array}{l}\text { Differential diagnosis| } \\
\text { symptom prevalence } \\
\text { study }\end{array}$ & Economic and decision analyses \\
\hline $1 \mathrm{a}$ & $\begin{array}{l}\text { SR (with homogeneity) } \\
\text { of RCTs }\end{array}$ & $\begin{array}{l}\text { SR (with homogeneity) } \\
\text { inception cohort studies; } \\
\text { CDR validated in diffe- } \\
\text { rent populations }\end{array}$ & $\begin{array}{l}\text { SR (with homogeneity) of } \\
\text { Level } 1 \text { diagnostic studies; } \\
\text { CDR with } 1 \text { b studies from } \\
\text { different clinical centers }\end{array}$ & $\begin{array}{l}\text { SR (with homogeneity) } \\
\text { of prospective cohort } \\
\text { studies }\end{array}$ & $\begin{array}{l}\text { SR (with homogeneity) of Level 1econo- } \\
\text { mic studies }\end{array}$ \\
\hline $1 b$ & $\begin{array}{l}\text { Individual RCT (with } \\
\text { narrow Confidence } \\
\text { Interval) }\end{array}$ & $\begin{array}{l}\text { Individual inception } \\
\text { cohort study with }>80 \% \\
\text { follow-up; CDR validated } \\
\text { in a single population }\end{array}$ & $\begin{array}{l}\text { Validating cohort study } \\
\text { with good reference stan- } \\
\text { dards; or CDR tested } \\
\text { within one clinical centre }\end{array}$ & $\begin{array}{l}\text { Prospective cohort } \\
\text { study with good } \\
\text { follow-up }\end{array}$ & $\begin{array}{l}\text { Analysis based on clinically sensible costs } \\
\text { or alternatives; systematic review (s) of } \\
\text { the evidence; and including multi-way } \\
\text { sensitivity analyses }\end{array}$ \\
\hline $2 a$ & $\begin{array}{l}\text { SR (with homogeneity) } \\
\text { of cohort studies }\end{array}$ & $\begin{array}{l}\text { SR (with homogeneity) of } \\
\text { either retrospective co- } \\
\text { hort studies or untreated } \\
\text { control groups in RCTs }\end{array}$ & $\begin{array}{l}\text { SR (with homogeneity) of } \\
\text { Level > } 2 \text { diagnostic studies }\end{array}$ & $\begin{array}{l}\text { SR (with homogeneity) } \\
\text { of Level } 2 \mathrm{~b} \text { and better } \\
\text { studies }\end{array}$ & $\begin{array}{l}\text { SR (with homogeneity) of Level > } 2 \text { eco- } \\
\text { nomic studies }\end{array}$ \\
\hline $2 b$ & $\begin{array}{l}\text { Individual cohort study } \\
\text { (including low quality } \\
\text { RCT; e. g., }<80 \% \text { follow- } \\
\text { up) }\end{array}$ & $\begin{array}{l}\text { Retrospective cohort } \\
\text { study or follow-up of un- } \\
\text { treated control patients } \\
\text { in an RCT; Derivation of } \\
\text { CDR or validated on split- } \\
\text { sample only }\end{array}$ & $\begin{array}{l}\text { Exploratory cohort study } \\
\text { with good reference stan- } \\
\text { dards; CDR after derivati- } \\
\text { on, or validated only on } \\
\text { split-sample or databases }\end{array}$ & $\begin{array}{l}\text { Retrospective cohort } \\
\text { study, or poor follow-up }\end{array}$ & $\begin{array}{l}\text { Analysis based on clinically sensible costs } \\
\text { or alternatives; limited review (s) of the } \\
\text { evidence, or single studies; and including } \\
\text { multi-way sensitivity analyses }\end{array}$ \\
\hline $2 c$ & $\begin{array}{l}\text { "Outcomes" Research; } \\
\text { Ecological studies }\end{array}$ & "Outcomes" Research & & Ecological studies & Audit or outcomes research \\
\hline $3 a$ & $\begin{array}{l}\text { SR (with homogeneity) } \\
\text { of case-control studies }\end{array}$ & & $\begin{array}{l}\text { SR (with homogeneity) of } \\
3 \mathrm{~b} \text { and better studies }\end{array}$ & $\begin{array}{l}\text { SR (with homogeneity) } \\
\text { of } 3 \mathrm{~b} \text { and better studies }\end{array}$ & $\begin{array}{l}\text { SR (with homogeneity) of 3b and better } \\
\text { studies }\end{array}$ \\
\hline $3 b$ & $\begin{array}{l}\text { Individual Case-Control } \\
\text { Study }\end{array}$ & & $\begin{array}{l}\text { Non-consecutive study; or } \\
\text { without consistently ap- } \\
\text { plied reference standards }\end{array}$ & $\begin{array}{l}\text { Non-consecutive co- } \\
\text { hort study; or very limi- } \\
\text { ted population }\end{array}$ & $\begin{array}{l}\text { Analysis based on limited alternatives or } \\
\text { costs, poor quality estimates of data, but } \\
\text { including sensitivity analyses incorpora- } \\
\text { ting clinically sensible variations }\end{array}$ \\
\hline 4 & $\begin{array}{l}\text { Case-series (and poor } \\
\text { quality cohort and ca- } \\
\text { se-control studies) }\end{array}$ & $\begin{array}{l}\text { Case-series (and poor } \\
\text { quality prognostic } \\
\text { cohort studies) }\end{array}$ & $\begin{array}{l}\text { Case-control study, poor } \\
\text { or non-independent refe- } \\
\text { rence standard }\end{array}$ & $\begin{array}{l}\text { Case-series or superse- } \\
\text { ded reference standards }\end{array}$ & Analysis with no sensitivity analysis \\
\hline 5 & $\begin{array}{l}\text { Expert opinion without } \\
\text { explicit critical apprai- } \\
\text { sal, or based on physio- } \\
\text { logy, bench research or } \\
\text { "first principles" }\end{array}$ & $\begin{array}{l}\text { Expert opinion without } \\
\text { explicit critical appraisal, } \\
\text { or based on physiology, } \\
\text { bench research or "first } \\
\text { principles" }\end{array}$ & $\begin{array}{l}\text { Expert opinion without ex- } \\
\text { plicit critical appraisal, or } \\
\text { based on physiology, } \\
\text { bench research or "first } \\
\text { principles" }\end{array}$ & $\begin{array}{l}\text { Expert opinion without } \\
\text { explicit critical apprai- } \\
\text { sal, or based on physio- } \\
\text { logy, bench research or } \\
\text { "first principles" }\end{array}$ & $\begin{array}{l}\text { Expert opinion without explicit critical } \\
\text { appraisal, or based on physiology, bench } \\
\text { research or "first principles" }\end{array}$ \\
\hline
\end{tabular}

Ende dieses Gruppenprozesses wurden die Empfehlungen von den stimmberechtigten Mandatsträgern (s. Kapitel 1.8) formal abgestimmt. Die Ergebnisse der jeweiligen Abstimmungen sind entsprechend den Kategorien in $\bullet$ Tab. 4 den Empfehlungen zugeordnet. In der Leitlinie werden zu allen evidenzbasierten Statements (s. Kapitel 2.2.5) und Empfehlungen das Evidenzlevel (s. Kapitel 2.2.1) der zugrunde liegenden Studien sowie bei Empfehlungen zusätzlich die Stärke der Empfehlung (Empfehlungsgrad) ausgewiesen. Hinsichtlich der Stärke der Empfehlung werden in dieser Leitlinie drei Empfehlungsgrade unterschieden ( $\bullet$ Tab.5), die sich auch in der Formulierung der Empfehlungen jeweils widerspiegeln.

\subsubsection{Klinische Beurteilung - Klassifizierung der Empfehlungsgrade}

(nach Empfehlungen des Europarats 2001)

In der Regel bestimmt der Evidenzklassifikation den Empfehlungsgrad. Abweichungen sind in begründeten Fällen möglich (s. weitere Kriterien in $\bullet$ Abb. 1).

\subsubsection{Klassifikation der Konsensusstärke ( $\bullet$ Tab.4)}

\subsubsection{Statements}

Als Statements werden Darlegungen oder Erläuterungen von spezifischen Sachverhalten oder Fragestellungen ohne unmittel-
Tab. 4 Klassifikation der Konsensusstärke.

\begin{tabular}{|l|l|}
\hline Konsensusstärke & Prozentuale Übereinstimmung \\
\hline starker Konsens & Zustimmung von $>95 \%$ der Teilnehmer \\
\hline Konsens & Zustimmung von $>75-95 \%$ der Teilnehmer \\
\hline Mehrheitliche Zustimmung & Zustimmung von $>50-75 \%$ der Teilnehmer \\
\hline Kein Konsens & Zustimmung von $<50 \%$ der Teilnehmer \\
\hline
\end{tabular}

Tab. 5 Schema der Empfehlungsgraduierung.

\begin{tabular}{|lll|}
\hline Empfehlungsgrad & Beschreibung & Syntax \\
\hline A & Starke Empfehlung & soll \\
\hline B & Empfehlung & sollte \\
\hline 0 & Empfehlung offen & kann \\
\hline
\end{tabular}

bare Handlungsaufforderung bezeichnet. Sie werden entsprechend der Vorgehensweise bei den Empfehlungen im Rahmen eines formalen Konsensusverfahrens verabschiedet und können entweder auf Studienergebnissen oder auf Expertenmeinungen beruhen. 


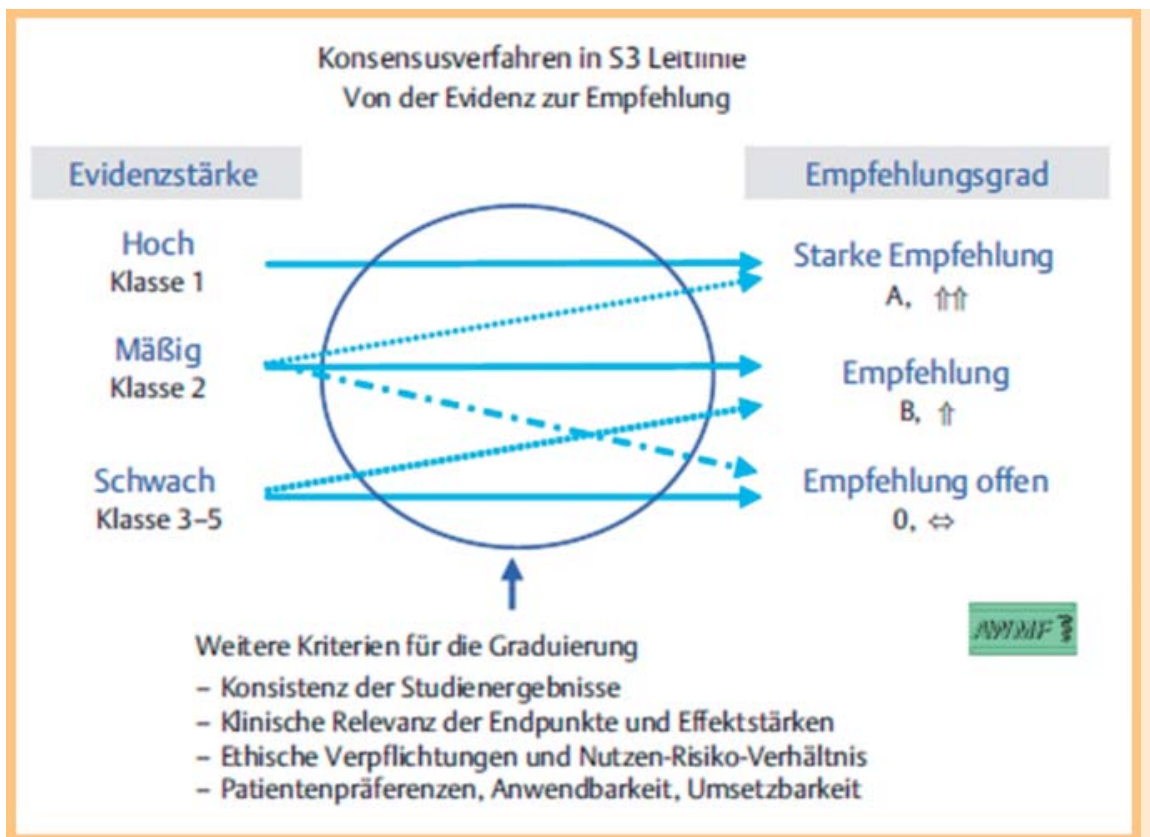

Abb. 1 Übersicht zur Graduierung von Empfehlungen.

\subsubsection{Good Clinical Practice (GCP)}

Als „Good Clinical Practice (GCP)“ werden Empfehlungen bezeichnet, zu denen keine Recherche nach Literatur durchgeführt wurde. In der Regel adressieren diese Empfehlungen Vorgehensweisen der guten klinischen Praxis, zu denen keine wissenschaftlichen Studien notwendig sind bzw. erwartet werden können. Für die Graduierung der GCPs wurden keine Symbole verwendet, die Stärke der Empfehlung ergibt sich aus der verwendeten Formulierung (soll/sollte/kann) entsprechend der Abstufung in - Tab. 5.

\subsubsection{Unabhängigkeit und Darlegung möglicher Interessenkonflikte}

Diese Leitlinie wurde durch die Deutsche Krebshilfe e.V. im Rahmen des Leitlinienprogramms Onkologie finanziert. Die Mittel wurden eingesetzt für Personalkosten (Medizinische Dokumentarin), Büromaterial, Literaturbeschaffung, Telefon- und Konsensuskonferenzen (Raummieten, Technik, Verpflegung, Moderatorenhonorar, Reisekosten der Teilnehmer). Die Reisekosten wurden entsprechend des Bundes-Dienstreisegesetzes bzw. nach den im Hochschulbereich üblichen Richtlinien erstattet. Die Aktualisierung der Leitlinie erfolgte in redaktioneller Unabhängigkeit von der finanzierenden Organisation. Alle Mitglieder der Leitliniengruppe legten eine schriftliche Erklärung zu evtl. bestehenden Interessenkonflikten vor (s. Leitlinienreport unter (http://leitlinienprogramm-onkologie.de/Leitlinien.7.0.html). Für ihre ausschließlich ehrenamtliche Arbeit, ohne die die S3Leitlinie nicht zu realisieren gewesen wäre, ist ihnen zu danken.

\subsection{Verwendete Abkürzungen}

$\begin{array}{ll}\text { AFAP } & \text { Attenuierte FAP } \\ \text { AHB } & \text { Anschlussheilbehandlung } \\ \text { ASS } & \text { Acetylsalicylsäure } \\ \text { AWMF } & \begin{array}{l}\text { Arbeitsgemeinschaft der Wissenschaftlichen Medi- } \\ \text { zinischen Fachgesellschaften }\end{array} \\ \text { BMI } & \text { Body-Mass-Index } \\ \text { CEA } & \text { Karzinoembryonales Antigen }\end{array}$

CT Computertomografie

CTC CT-Kolonografie

CU Colitis ulcerosa

DGE Deutsche Gesellschaft für Ernährung

FAP Familiäre adenomatöse Polyposis

FOBT Fäkaler okkulter Bluttest

HNPCC Hereditäres kolorektales Karzinom ohne Polyposis

IEN Intraepitheliale Neoplasie

iFOBT/FIT Immunologischer FOBT

IHC Immunhistochemische Untersuchung

KRK Kolorektales Karzinom

LITT Laserinduzierte interstitielle Thermotherapie

LL Leitlinie

MAP MUTYH-assoziierte Polyposis

MMR Mismatch-repair-Gen

MSA Mikrosatellitenanalyse

MSCT Mehrzeilen-CT

MSI Mikrosatelliteninstabilität

MSI-H Mikrosatelliteninstabilität hoch (high)

MSI-L Mikrosatelliteninstabilität gering (low)

MSS Mikrosatellitenstabilität

ÖGD Ösophagogastroduodenoskopie

OL Leitstelle Onkologie der DKG

OR Odds Ratio

PCI Peritoneal cancer index

PET Positronen-Emissionstomographie

PJS Peutz-Jeghers-Syndrom

PSC Primär sklerosierende Cholangitis

RCT Randomisierte kontrollierte Studie

RFA Radiofrequenzablation

RR Relatives Risiko

SIRT Selektive interne Radiotherapie

SR Systematische Übersichtsarbeit

WHO Weltgesundheitsorganisation 


\subsection{Redaktioneller Hinweis}

Die aktuelle Überarbeitung betrifft die Themenkomplexe I, II, III, V und VIII bzw. Kapitel 3, 4, 5, 7 und 10 sowie einzelne Abschnitte aus 6, 8 und 9. Zu den Empfehlungen ist jeweils das Datum der letzten Überarbeitung in einer Klammer aufgeführt. Bei den überarbeiteten Kapiteln stehen die Literaturhinweise sowohl im Hintergrundtext, als auch in den Empfehlungskästen. Bei den 2007/2008 aktualisierten Kapiteln sind die Referenzen lediglich im Fließtext vermerkt.

In einem Pilotprojekt wurde eine externe Expertin (PD Dr. A. Riphaus) beauftragt, exemplarisch für die Themen Prävention und Nachsorge eine Literatursuche zu geschlechtsspezifischen Unterschieden durchzuführen. Die systematische Recherche ergab, dass keine Empfehlung zu genderspezifisch unterschiedlicher Behandlung abgegeben werden kann.

Aus diesem Grund wird in dieser Leitlinie der Begriff „Patienten“ für Frauen und Männer verwendet. Dies dient lediglich der leichteren Lesbarkeit des Textes.

\section{Prävention asymptomatische Bevölkerung}

\subsection{Lebensgewohnheiten}

\subsection{Empfehlung (2013)}

Zur Risikoreduktion eines kolorektalen Karzinoms sollten regelmä-

ßig körperliche Aktivitäten durchgeführt werden.

Empfehlungsgrad B, Level of Evidence 2a, Evidenz aus Aktualisierungsrecherche $^{1}$ : [1 -12], starker Konsens

\subsection{Empfehlung (2013)}

Zur Risikoreduktion eines kolorektalen Karzinoms sollte eine Gewichtsreduktion bei übergewichtigen Personen angestrebt werden. Empfehlungsgrad B, Level of Evidence 2a, Evidenz aus Aktualisierungsrecherche: [1, 8, 13-18], starker Konsens

\subsection{Empfehlung (2013)}

Die Bevölkerung soll zum Verzicht auf Tabakrauchen angehalten werden.

Empfehlungsgrad A, Level of Evidence 2a, Evidenz aus Aktualisierungsrecherche: [1, 10,19-25], starker Konsens

\section{Hintergrund}

Personen mit höherem körperlichen Aktivitätsgrad haben in Querschnittsuntersuchungen und prospektiven Kohortenstudien weniger Kolonpolypen (Adenome) und ein um bis zu $30 \%$ geringeres Karzinomrisiko. Bereits 30 - 60 min tägliche moderate körperliche Aktivität gehen mit einem verringerten Karzinomrisiko einher [1 - 12].

Es findet sich eine positive Assoziation zwischen dem Auftreten von Kolonpolypen (Adenomen) und kolorektalen Karzinomen und einem höheren BMI, als auch einer Zunahme des Bauchumfangs. Dieser Effekt ist ab einem BMI von $>25 \mathrm{~kg} / \mathrm{m}^{2}$ nachweisbar, nimmt linear mit dem BMI zu und ist stärker bei Männern als bei Frauen ausgeprägt. Bei übergewichtigen Personen insbesondere bei stammbetonter Adipositas war das Risiko für ein Kolonkarzinom bis zu zweifach erhöht [18]. Es ist unklar, ob die Risikoerhöhung durch das Übergewicht, veränderte Hormonspiegel, die er-

\footnotetext{
${ }^{1}$ Systematische Recherche mit eingeschränktem Zeitintervall (ab 2003, entsprechend dem Endpunkt der De-novo-Suche für die letzte Aktualisierung).
}

höhte Kalorienaufnahme oder durch die fehlende körperliche Aktivität bedingt ist $[1,8,13-18]$.

Rauchen ist mit einem zweifach erhöhten Risiko für Kolonadenome und erhöhtem Risiko für kolorektale Karzinome assoziiert $[1,10,19-25]$.

\subsection{Ernährungsempfehlungen}

\subsection{Statement (2013)}

Eine spezifische Diätempfehlung zur Reduktion des KRK-Risikos kann derzeit nicht gegeben werden.

Level of Evidence 2b, Evidenz aus Aktualisierungsrecherche: [26 - 32], Konsens

\subsection{Konsensbasierte Empfehlung (2013)}

Es sollten die allgemeinen Ernährungsempfehlungen der DGE befolgt werden.

GCP, Konsens

\section{Hintergrund}

In einer ausführlichen Literaturübersicht aus dem Jahre 2010 wurde ein Zusammenhang zwischen einer „gesunden“ Ernährungsweise bzw. einer „ungesünderen“ Ernährungsweise und dem kolorektalen Karzinom als wahrscheinlich angesehen. Eine „gesunde“ Ernährungsweise wurde von den Autoren gekennzeichnet durch einen hohen Konsum von Obst und Gemüse sowie einer verminderten Aufnahme von rotem und verarbeitetem Fleisch, wohingegen eine „ungesündere“ Ernährungsweise durch eine hohe Aufnahme von rotem und verarbeitetem Fleisch, Kartoffeln und raffinierter Stärke charakterisiert wurde [26]. In Originalpublikationen der letzten Jahre wurde wiederholt eine Assoziation zwischen Ernährungsfaktoren und der Ausbildung eines KRK beobachtet, die mit einer Evidenzstärke zwischen 2b und $4 \mathrm{zu}$ bewerten sind [27 - 30]. Allerdings gibt es auch Studien, in denen keine Korrelation zwischen Ernährungsfaktoren und KRK beobachtet wurde [31, 32]. Dabei handelt es sich um Assoziationen, nicht um Interventionsstudien. Ob solche Zusammenhänge eine spezifische Diätempfehlung zur KRK Prävention rechtfertigen, wurde bislang nicht untersucht. Deshalb kann derzeit trotz der skizzierten Zusammenhänge keine spezifische Diätempfehlung abgegeben werden. Es wird stattdessen empfohlen, dass zur Risikoreduktion eines Karzinoms die aktuellen Ernährungsempfehlungen der DGE berücksichtigt werden. Die Assoziationen zwischen der Aufnahme bestimmter Nahrungsstoffe und dem KRKRisiko wird im Folgenden genauer dargestellt. In diesem Zusammenhang ist auch hervorzuheben, dass eine Ernährungsweise, die keine Gewichtszunahme bewirkt, anzuraten ist (s. Kapitel 3.1).

\subsection{Empfehlung (2013)}

Zur Risikosenkung eines KRK sollte die Ballaststoffaufnahme möglichst $30 \mathrm{~g}$ pro Tag betragen.

Empfehlungsgrad B, Level of Evidence 2a, Evidenz aus Aktualisierungsrecherche: [33 - 37], Konsens

\section{Hintergrund}

Trotz kontroverser Datenlage ist die Evidenz ausreichend, um eine ballaststoffreiche Ernährung von $30 \mathrm{~g} / \mathrm{Tag}$ zu empfehlen [33 - 37]. Eine aktuelle britische Studie, in der Daten von sieben Kohortenstudien zusammengefasst wurden, wies nach, dass eine inverse Korrelation zwischen Ballaststoffaufnahme und Karzinomrisiko besteht. Der Vergleich des täglichen Ballaststoffkonsums von 10 und $24 \mathrm{~g}$ zeigte in dieser Arbeit, dass ein höherer Konsum mit einem um $30 \%$ reduzierten Kolonkarzinomrisiko assoziiert ist [33]. 
In einer anderen Studie, in der 13 prospektive Kohortenstudien zusammengefasst wurden, zeigten sich ähnliche Ergebnisse. Obwohl das pooling project of prospective studies of diet and cancer noch größere Spannbreiten zwischen der niedrigsten und höchsten Quintile der Ballaststoffaufnahme aufwies, wurde eine signifikante inverse Korrelation zwischen dem Ballaststoffkonsum und dem Krebsrisiko nach alterskorrigierter Analyse, jedoch nicht nach Adjustierung für weiterer ernährungsbedingte Risikofaktoren, beobachtet [36]. Die nur eingeschränkt positiven Daten könnten darauf zurückzuführen sein, dass lediglich zu Studienbeginn eine Erfassung der Ballaststoffaufnahme erfolgte, die möglicherweise inkorrekt den langfristigen Konsum widerspiegelt. Trotz der eingeschränkten Ergebnisse sind die verbleibenden Aussagen sehr robust, weil sie auf großen Personenkollektiven basieren. Deshalb wurde der Empfehlungsgrad B festgelegt.

\subsection{Empfehlung (2013)}

Zur Reduktion des Risikos eines KRK sollte der Alkoholkonsum limitiert werden.

Empfehlungsgrad B, Level of Evidence 2a, Evidenz aus Aktualisierungsrecherche: [38-41], Konsens

\section{Hintergrund}

Es besteht eine positive Korrelation zwischen einem hohen Alkoholkonsum und der Entstehung eines KRK [38 - 41] insbesondere bei Menschen mit geringer Folsäure- und/oder Methioninaufnahme [39]. Abstinenzler und Personen mit einem geringen Alkoholkonsum weisen ein signifikant geringeres Karzinomrisiko auf [38-41]. Eine Metaanalyse von 14 prospektiven Kohortenstudien zeigte, dass bereits eine Alkoholaufnahme von $100 \mathrm{~g}$ wöchentlich mit einem 15\%igen Anstieg sowohl des Kolon- als auch des Rektumkarzinom-Risikos assoziiert ist [41]. Das Risiko korreliert mit der Menge des aufgenommenen Alkohols und nicht mit der Art des alkoholischen Getränks [39].

\subsection{Empfehlung (2013)}

Rotes bzw. verarbeitetes Fleisch sollte nur in geringen Mengen (nicht täglich) konsumiert werden.

Empfehlungsgrad B, Level of Evidence 2a, Evidenz aus Aktualisierungsrecherche: [37, 42 - 46], Konsens

\section{Hintergrund}

Ein hoher Konsum von rotem Fleisch (Rind, Kalb, Schwein und Lamm) und verarbeitetem Fleisch ist mit einem erhöhten Risiko für ein KRK assoziiert [37, 42-46]. Zwischen dem Verzehr von Geflügel und/oder -produkten besteht keine positive Korrelation [45]. Die positive Assoziation wird am ehesten durch die Verarbeitung und die Zubereitungsart verursacht, wie Daten des Prostate, Lung, Colorectal, and Ovarian cancer trials zeigen. Die regelmäßige Aufnahme von insbesondere durchgegartem rotem Fleisch, Speck (Bacon) und Würstchen korrelierte mit einer signifikanten Erhöhung des Risikos zur Ausbildung eines KRK [46].

\subsection{Statement (2013)}

$\mathrm{Zu}$ erhöhtem Fischkonsum kann keine Empfehlung abgegeben werden.

Level of Evidence 2a, Evidenz aus Aktualisierungsrecherche: [42, 44, 45, 47 - 49], Konsens

\section{Hintergrund}

In einer Metaanalyse von Geelen und Kollegen, die 19 Kohortenstudien zusammenfasst, wurde der Einfluss des Fischverzehrs auf das KRK-Risiko untersucht. Der Vergleich des höchsten wöchentlichen Fischkonsums mit dem geringsten zeigte, dass ein höherer Konsum mit einem um 12 \% reduzierten Krebsrisiko assoziiert ist. Die Korrelation war umso ausgeprägter je höher die Differenz zwischen der größten und geringsten Fischaufnahme war [47]. Die Datenlage insgesamt ist allerdings widersprüchlich, was wahrscheinlich darauf zurückzuführen ist, dass in den verschiedenen Studien unterschiedlich hohe Fischverzehrmengen miteinander verglichen wurden [42, 44, 45, 47 - 49] Trotz der Vermutung, dass vermehrter Fischkonsum das KRK-Risiko leicht senken kann, wird aufgrund der nicht eindeutigen Datenlage derzeit keine Empfehlung zum erhöhten Fischkonsum abgegeben.

\subsection{Konsensbasierte Empfehlung (2013)}

Ein Zusammenhang zwischen der Aufnahme von Kaffee/Tee und der Reduktion des Risikos für ein KRK ist nicht belegt, deshalb gibt es keine Empfehlung zu Kaffee- oder Teekonsum.

GCP, starker Konsens

\section{Hintergrund}

Zu diesem Thema liegen drei Metaanalysen vor, die keine Korrelation zwischen dem Konsum von Kaffee und/oder Tee und dem KRK-Risiko beobachtet haben [ 50 - 52].

\subsection{Konsensbasierte Empfehlung (2013)}

Trotz der nicht eindeutigen Datenlage zur Prävention des KRK sollten Obst und Gemüse vermehrt konsumiert werden (5 Portionen am Tag).

GCP, Konsens

\section{Hintergrund}

In einer Fallkontrollstudie und einer Kohortenstudie wurde eine inverse Korrelation zwischen einem vermehrten Obst- und Gemüsekonsum und einer Reduzierung des KRK-Risikos beobachtet [53, 54]. Allerdings wurde in einer Metaanalyse gezeigt, dass ein erhöhter Obst- und Gemüseverzehr lediglich mit einem um 6-9\% reduzierten Kolonkarzinomrisiko assoziiert ist. Eine stärkere inverse Korrelation wurde beim distalen Kolonkarzinom beobachtet [55]. Unklar ist jedoch, welche Bestandteile (Ballaststoffe, sekundäre Pflanzenstoffe) diesen protektiven Effekt haben. Trotz nicht eindeutiger Datenlage hinsichtlich Reduktion des KRK Risikos wird eine Förderung des Obst- und Gemüsekonsums als wünschenswert angesehen, da ein regelmäßiger Obst- und Gemüseverzehr wahrscheinlich das allgemeine Krankheitsrisiko senkt.

\subsection{Konsensbasiertes Statement (2013)}

Es gibt keinen Zusammenhang zwischen Nahrungszubereitung oder Nahrungsfettkomponenten und KRK-Risiko.

GCP, Konsens

\section{Hintergrund}

Es wurde wiederholt diskutiert, ob die Nahrungszubereitung bzw. der durch Nahrungszubereitungsmethoden möglicherweise erhöhte Anteil an potenziell schädlichen Fettsäuren, wie z.B. Transfettsäuren, das KRK-Risiko erhöhen kann. Die Literaturdaten dazu sind spärlich und uneinheitlich, sodass angenommen werden muss, dass es keinen klaren Zusammenhang gibt. Dies wurde in einer neueren prospektiven, populationsbasierten Kohortenstudie in den USA untersucht. In dieser Studie an über 35000 Frauen konnte bestätigt werden, dass Transfettsäuren das KRK-Risiko nicht erhöhen [56]. 
Darüber hinaus gibt es keine spezifischen Empfehlungen zum Fettkonsum hinsichtlich Reduktion des KRK Risikos. Zu dieser Thematik liegen mehrere Studien vor, die keinen Zusammenhang zwischen dem Fettkonsum und der Ausbildung eines KRK gefunden haben. Ein Effekt von Kofaktoren, wie z. B. Zufuhr von rotem Fleisch oder Zubereitungsart, kann nicht hinreichend abgetrennt werden [30, 31, 37, 47, 57 - 59].

\subsection{Statement (2013)}

Es gibt keinen Zusammenhang zwischen der Aufnahme von Acrylamid und KRK-Risiko.

Level of Evidence 2b, de novo: [60 -63], starker Konsens

\section{Hintergrund}

In einer in Schweden durchgeführten prospektiven, populationsbasierten Kohortenstudie an mehr als 45000 Männern konnte mittels Food Frequency Questionnaire (FFQ) gezeigt werden, das es keinen Zusammenhang zwischen Acrylamid in der Nahrung und KRK-Risiko gibt [60]. Diese Studie bestätigt frühere Untersuchungen, nach denen bei Männern wie Frauen kein Zusammenhang zwischen Acrylamid und KRK-Entstehung besteht [61 - 63].

\subsection{Mikronährstoffe}

\subsection{Empfehlung (2013)}

Es gibt derzeit keine gesicherten Daten zur wirksamen Prävention des kolorektalen Karzinoms durch Mikronährstoffe. Die Einnahme von Supplementen dieser Substanzen im Rahmen der Primärprävention des kolorektalen Karzinoms sollte daher nicht erfolgen.

Diese Empfehlung gilt für...

...Vitamine...

Empfehlungsgrad B, Level of Evidence 2b, Evidenzgrundlage de novo: [64]

... inklusive $\beta$-Carotin

Empfehlungsgrad B, Level of Evidence 3b, Evidenzgrundlage de novo: [64]

... Vitamin C, Vitamin D, Vitamin E

Empfehlungsgrad B, Level of Evidence 4, Evidenzgrundlage de novo: [64-66]

... und Folsäure

Empfehlungsgrad B, Level of Evidence 1a, Evidenzgrundlage de novo: [67-71]

Weiterhin gelten diese Empfehlungen für Kalzium

Empfehlungsgrad B, Level of Evidence 1b, Aktualisierungsrecherche: $[65,66,72-74]$

\section{... Magnesium}

Empfehlungsgrad B, Level of Evidence 2b, Evidenzgrundlage de novo: [75]

... und Selen.

Empfehlungsgrad B, Level of Evidence 2b, Evidenzgrundlage de novo: [76, 77]

Jeweils starker Konsens

\section{Hintergrund}

Die folgenden Ausführungen beziehen sich auf Supplemente von Mikronährstoffen, teilweise in pharmakologischer, d.h. durch Aufnahme entsprechender Lebensmittel (Obst, Gemüse, Milchprodukte), oftmals nicht erreichbarer Dosierung.

Für Kalzium konnte ein moderater, klinisch nicht relevanter Hemmeffekt auf die Rekurrenz von Kolonadenomen nachgewiesen werden [72 - 74]. Hingegen ist die Datenlage für eine risikosenkende Wirkung von Kalzium oder Vitamin D, allein oder in Kombination, hinsichtlich des KRK selbst nicht überzeugend [65, 66].
Es gibt keine Evidenz für die Annahme, dass Beta-Carotin, Vitamin A oder Vitamin E das Risiko des kolorektalen Karzinoms vermindern könnten. Im Gegenteil konnte in einer Metaanalyse [64] gezeigt werden, dass die Supplementierung der vorgenannten Vitamine, allein oder in Kombination gegeben, mit einer erhöhten generellen Mortalität assoziiert ist.

Es ist nicht eindeutig belegt, dass die Einnahme von hohen Dosen an Vitamin C das Risiko für das KRK vermindert.

Ein das KRK-Risiko senkender Effekt von Folsäure konnte bislang nicht zweifelsfrei nachgewiesen werden [67]. Studien zur Rekurrenz von Kolonadenomen führten zu divergenten Ergebnissen [68-71]

Eine Interventionsstudie mit einem Selen-Supplement und dem Hauptzielkriterium „KRK-Inzidenz“ wurde bislang nicht durchgeführt. Die Korrelation niedriger Selen-Konzentrationen im Serum mit einem erhöhten Adenomrisiko ist nicht ausreichend, um eine Empfehlung zur Selensupplementierung auszusprechen [76, 77].

\subsection{Medikamente}

\subsection{Empfehlung (2013)}

COX-2-Hemmer sollen in der asymptomatischen Bevölkerung nicht zur Prophylaxe des kolorektalen Karzinoms eingenommen werden. Empfehlungsgrad A, Level of Evidence 3b, de novo: [78-81], Konsens

\subsection{Empfehlung (2013)}

Statine sollten nicht zur Primärprophylaxe des KRK eingesetzt werden.

Empfehlungsgrad B, Level of Evidence 2b, de novo: [82], starker Konsens

\subsection{Empfehlung (2013)}

Acetylsalicylsäure soll nicht zur Primärprävention des kolorektalen Karzinoms in der asymptomatischen Bevölkerung eingenommen werden.

Empfehlungsgrad A, Level of Evidence 2a, Leitlinienadaptation: [83 - 86], Konsens

\subsection{Empfehlung (2013)}

Eine Hormontherapie ${ }^{2} z u r$ Risikoreduktion eines kolorektalen Karzinoms bei Frauen soll nicht gegeben werden.

Empfehlungsgrad A, Level of Evidence 1a, Leitlinienadaptation: $[87,88]$, starker Konsens

\section{Hintergrund}

Für die Einnahme von Cyclooxygenase-2(COX-2)-Inhibitoren zur Primärprävention des kolorektalen Karzinoms liegt eine positive Fall-Kontroll-Studie, aber keine randomisierte Studie vor [78]. Drei randomisierte Studien zur Sekundärprävention mit Celecoxib bzw. Rofecoxib nach Polypektomie zeigen übereinstimmend, dass COX-2-Inhibitoren das Rezidivrisiko eines kolorektalen Adenoms signifikant senken [79-81]. In allen drei Studien zeigte sich jedoch eine deutlich erhöhte kardiovaskuläre Morbidität.

Eine aktuelle Metaanalyse von Fallkontroll-, Kohorten- und randomisierten Studien belegt einen statistisch signifikanten, aber nur geringen Effekt von Statinen in der Primärprävention des kolorektalen Karzinoms [82]. In einer Phase-III-Studie zur Sekundärprävention von Kolonadenomen senkte Ursodesoxycholsäure

\footnotetext{
${ }^{2}$ Da der Begriff „Hormonersatztherapie“ irreführend ist, wird in der Leitlinie nur der Begriff „Hormontherapie“ verwendet.
} 
signifikant nur das Risiko für Adenome mit hochgradigen Dysplasien, aber nicht für Adenome insgesamt [89]. Prospektive Studien zur Primärprävention von Adenomen mittels Ursodesoxycholsäure liegen nicht vor.

Eine Metaanalyse von 2 großen randomisierten Studien mit zusammen über 7500 Teilnehmern zeigt, dass die Einnahme von 300 mg oder mehr Acetylsalicylsäure pro Tag für 5 Jahre mit einer Latenz von 10 und mehr Jahren das Risiko, an einem kolorektalen Karzinom zu erkranken, senkt [84]. Eine andere Metaanalyse, die insgesamt 8 randomisierte Studien mit zusammen 25570 Teilnehmern analysiert hat, weist nach, dass die tägliche Einnahme von mindestens $75 \mathrm{mg}$ ASS mit einer Latenz von 10 Jahren die Mortalität am kolorektalen Karzinom senkt [85]. Für die Einnahme nicht steroidaler Antirheumatika (NSAR) wird in Kohortenund Fallkontrollstudien eine Erniedrigung der Inzidenz des kolorektalen Karzinoms beschrieben. Diese Befunde wurden allerdings noch nicht durch randomisierte Studien bestätigt [84]. Aufgrund der gehäuften Inzidenz von gastrointestinalen Blutungen unter ASS [86] und der fehlenden Bewertung der Nutzen-/Risikorelation folgt die Leitliniengruppe der Leitlinie der U.S. Preventive Services Task Force zum Einsatz von ASS oder NSAR zur Primärprävention des kolorektalen Karzinoms aus dem Jahr 2007, die empfiehlt, ASS und NSAR nicht zur Primärprävention des kolorektalen Karzinoms einzusetzen [83].

Eine Hormontherapie kann das kolorektale Karzinomrisiko reduzieren [88]. Aufgrund der erhöhten Inzidenz von unerwünschten Wirkungen, insbesondere venöser Thromboembolien, kann die Hormontherapie bei postmenopausalen Frauen aber nicht zur Primärprävention des kolorektalen Karzinoms empfohlen werden. Hier folgt die Leitliniengruppe der Leitlinie der U.S. Preventive Services Task Force zum Einsatz von Hormontherapie bei postmenopausalen Frauen [87] und der Leitlinie Hormontherapie in der Peri- und Postmenopause der Deutschen Gesellschaft für Gynäkologie und Geburtshilfe [90].

\section{Vorsorge/Früherkennung asymptomatische Bevölkerung \\ $\nabla$}

Asymptomatische Bevölkerung - Definition

Personen, die keiner Risikogruppe für das Auftreten eines kolorektalen Karzinoms angehören.

\subsection{Vorsorge-/Früherkennungs-Alter}

\subsection{Konsensbasierte Empfehlung (2013)}

Mit der Darmkrebs-Vorsorge/-Früherkennung für die asymptomatische Bevölkerung sollte ab dem Alter von 50 Jahren begonnen werden. Eine obere Altersbegrenzung für die Darmkrebs-Vorsorge/-Früherkennung kann bei steigender Lebenserwartung nicht gegeben werden. Hier ist eine individuelle Entscheidung unter Berücksichtigung der Begleiterkrankungen angezeigt.

GCP, starker Konsens

\section{Hintergrund}

Die KRK-Inzidenz steigt ab einem Alter von 50 Jahren deutlich an $[91,92]$. In einer prospektiven Koloskopiestudie zeigte sich eine deutlich niedrigere Nachweisrate fortgeschrittener Adenome bei 40- bis 49-jährigen (3,5\%) [93]. Von großer Bedeutung ist die Identifikation von Personen mit erhöhtem KRK-Risiko, für die gesonderte Empfehlungen gelten (s. Kapitel 5)

Zur Altersbegrenzung der Darmkrebsvorsorge existieren keine prospektiven Studien. In den FOBT-Studien wurden nur Personen bis 75 Jahren eingeschlossen. Die US Preventive Task Force rät von einer Vorsorge/Früherkennung von Personen über 85 Jahren $\mathrm{ab}$ und hält fest, dass eine Vorsorge/Früherkennung bei Personen zwischen 76 und 85 Jahren generell nicht durchgeführt werden sollte, in individuellen Fällen jedoch überlegt werden könnte [94]. Die Inzidenz fortgeschrittener Neoplasien nimmt mit dem Alter zu [95]. Endoskopische Untersuchungen scheinen auch bei älteren Patienten sicher durchführbar zu sein [96], jedoch nahm in einer Kohortenstudie die Komplikationsrate mit dem Alter zu [97]. In einer Studie war die relative 5-Jahres-Überlebensrate nach kurativer Operation eines kolorektalen Karzinoms für Patienten über 74 Jahre vergleichbar mit der von Patienten zwischen 50 und 74 Jahren [98]. Die Sinnhaftigkeit der Durchführung einer KRK-Früherkennung/Vorsorge sollte daher individuell in Abhängigkeit des „biologischen Alters“ und unter Berücksichtigung vorhandener Begleiterkrankungen überprüft werden. Zum Nutzen/Risikoverhältnis der Darmkrebs- Vorsorge/-Früherkennung in verschiedenen Altersgruppen existiert keine ausreichende Datenlage.

\subsection{Untersuchungsverfahren für die}

Darmkrebsfrüherkennung/-vorsorge

Bei den zur Verfügung stehenden Verfahren muss zwischen solchen, die vorwiegend Karzinome nachweisen (FOBT, genetische Stuhltests, M2-PK) und solchen, die zusätzlich in der Lage sind Adenome nachzuweisen (Koloskopie, Sigmoidoskopie, CT-Kolonografie, Kapselendoskopie) unterschieden werden. Folgende Verfahren werden besprochen:

- Koloskopie

- Sigmoidoskopie

- FOBT

- Genetische und andere Stuhltests

- CT-Kolonografie

- Kapselendoskopie

\subsubsection{Endoskopische Verfahren}

Von allen Maßnahmen zur Früherkennung kolorektaler Neoplasien besitzt die Koloskopie die höchste Sensitivität und Spezifität (Goldstandard). Endoskopische Maßnahmen sind als einzige diagnostisch und therapeutisch und haben den Vorteil, dass durch sie auch nichtblutende Karzinome und Adenome mit hoher Sensitivität nachgewiesen werden können. Durch die Abtragung von Adenomen kann zudem die Entstehung von Karzinomen effektiv verhindert (Unterbrechung der Adenom-Karzinomsequenz) [99, 100] und wie kürzlich gezeigt wurde, auch die KRK-bedingte Mortalität gesenkt werden [101].

In einzelnen randomisierten Studien konnte zudem gezeigt werden, dass trotz geringerer Teilnahmerate im Vergleich zum FOBT durch sowohl Sigmoidoskopie [102] als auch Koloskopie [103] in einer Intention-to-screen-Analyse mehr fortgeschrittene Neoplasien detektiert werden, was v. a. durch die deutlich höhere Sensitivität für fortgeschrittene Adenome bedingt war.

\subsubsection{Koloskopie}

\subsection{Empfehlung (2013)}

Die komplette qualitätsgesicherte Koloskopie besitzt die höchste Sensitivität und Spezifität für das Auffinden von Karzinomen und Adenomen und sollte daher als Standardverfahren für die KRK-Vorsorge/-Früherkennung eingesetzt werden. Bei unauffälligem Befund sollte die Koloskopie nach 10 Jahren wiederholt werden. Zur Durchführung wird auf die Krebsfrüherkennungsrichtlinie [104] verwiesen, die digitale rektale Untersuchung ist hierbei obligat. Bei 
Personen, die an der Koloskopie- Vorsorge/-Früherkennung entsprechend dieser Richtlinie teilnehmen, erübrigt sich das FOBT-Vorsorge/Früherkennungsverfahren.

Empfehlungsgrad B, Level of Evidence, 3b, de novo: [97, 105 120], starker Konsens

\section{Hintergrund}

Für den Einsatz der Koloskopie in der Früherkennung bzw. Vorsorge kolorektaler Karzinome existieren anders als für den FOBT und die Sigmoidoskopie keine Ergebnisse aus randomisierten Studien. Derartige Studien sind in Europa und den USA begonnen worden, Ergebnisse werden jedoch erst in frühestens 15 Jahren vorliegen. Dennoch wird der Einsatz der Koloskopie durch indirekte Evidenz unterstützt. In der externen Literatursuche wurde eine Arbeit aus Deutschland identifiziert, in der Patienten mit kolorektalen Karzinomen, die im Rahmen einer Vorsorgekoloskopie entdeckt worden waren, eine bessere Prognose aufwiesen als Karzinompatienten, die aufgrund von Beschwerden koloskopiert worden waren [105]. In einer weiteren Arbeit aus den USA wies eine Kohorte von 715 Personen nach Vorsorgekoloskopie eine signifikante Senkung von KRK-bedingter Mortalität und Inzidenz im Vergleich zu einem Vergleichskollektiv auf [106].

In großen Kohorten u. a. aus Deutschland konnte gezeigt werden, dass durch die Koloskopie eine hohe Anzahl an Karzinomen im frühen Stadium sowie Adenomen im gesamten Dickdarm entdeckt werden kann [97]. In Deutschland befinden sich etwa 1/3 der im Rahmen der Vorsorgekoloskopie detektierten Karzinome proximal des Colon descendens [97]. In anderen Studien wiesen 46 bis 52 \% der Patienten mit proximalen Neoplasien keine zusätzlichen distalen Adenome auf [107, 108]. Bei diesen Patienten wäre eine Diagnose der Neoplasien mittels Sigmoidoskopie unmöglich.

Die Ergebnisse der Fallkontrollstudien sowie der randomisierten UK-Studie zur Sigmoidoskopie, die jeweils eine Senkung der Karzinominzidenz und -mortalität zeigen konnten, sollten auf die Koloskopie übertragbar sein [109 - 112], wobei der Effekt im proximalen Kolon geringer zu sein scheint als im distalen Kolon [113 - 115]. Auch der protektive Effekt der FOBT-Studien beruht letztendlich auf der Abklärung positiver Tests mittels Koloskopie. Die Komplikationsrate der Untersuchung in Deutschland war in einer Studie auf freiwilliger Basis sehr gering [116]. Diese Ergebnisse konnten kürzlich bestätigt werden [97]. Es ist jedoch von einer Untererfassung der Komplikationen auszugehen, da Spätkomplikationen nur inkomplett erfasst werden. Tandemuntersuchungen haben gezeigt, dass größere Adenome nur selten (0 $6 \%$ übersehen werden [117].

Es wird davon ausgegangen, dass eine unauffällige Koloskopie nach 10 Jahren wiederholt werden sollte. So fanden sich 5,5 Jahre nach einer unauffälligen Koloskopie keine Karzinome und weniger als $1 \%$ fortgeschrittene Neoplasien [118]. Fallkontrollstudien legen nahe, dass das Risiko auch noch mehr als 10 Jahre nach einer unauffälligen Koloskopie sehr niedrig ist [113, 119].

Von entscheidender Bedeutung ist, dass die Koloskopie mit höchstmöglicher Qualität durchgeführt wird. Für die Durchführung der Koloskopie existieren in Deutschland klare Richtlinien [104].

\subsubsection{Sigmoidoskopie}

\subsection{Empfehlung (2013)}

Eine qualitätsgesicherte Sigmoidoskopie sollte Personen, die die Koloskopie als Vorsorge-/Früherkennungsmaßnahme ablehnen, angeboten werden.
Empfehlungsgrad B, Level of Evidence 2b, de novo: [121], starker Konsens

\subsection{Empfehlung (2013)}

Zur möglichen Detektion proximaler Karzinome sollte zusätzlich zur Sigmoidoskopie eine jährliche FOBT-Durchführung erfolgen.

Empfehlungsgrad B, Level of Evidence 3b, de novo: [109, 112, 122 - 129], starker Konsens

\section{Hintergrund}

Die Effektivität der Sigmoidoskopie als Früherkennungs-/Vorsorge-Methode für das KRK ist gesichert. In einer randomisierten Studie aus England wurde im Vergleich zu keinem Screening durch eine einmalige Sigmoidoskopie nach einer Nachbeobachtungszeit von 11,2 Jahren eine Senkung der KRK-bedingten Mortalität um $43 \%$ und eine Senkung der Inzidenz kolorektaler Karzinome um $33 \%$, die distaler Karzinome um 50\% beobachtet [121].

Es ist jedoch zu bedenken, dass im Rahmen einer Sigmoidoskopie nicht alle Darmabschnitte eingesehen werden können. Entsprechend wurde in der Sigmoidoskopiestudie die Inzidenz proximaler Karzinome nicht beeinflusst. Hier ist die Koloskopie der Sigmoidoskopie überlegen.

Der protektive Effekt einer Sigmoidoskopie für distale Neoplasien scheint 6 bis 10 Jahre [112, 122], in einer Studie sogar 16 Jahre anzuhalten [123]. In einer Untersuchung an 9417 Personen, die 3 Jahre nach unauffälliger Sigmoidoskopie einer erneuten Sigmoidoskopie unterzogen wurden, fanden sich jedoch bei $0,8 \%$ der Patienten im distalen Kolon ein fortgeschrittenes Adenom oder Karzinom [124]. In einer weiteren Studie mit 2146 Teilnehmern mit unauffälliger Sigmoidoskopie wurde ein Kontrollintervall von 3 und 5 Jahren verglichen [125]. Die Rate an fortgeschrittenen Neoplasien unterschied sich nicht signifikant $(0,9 \%$ vs. $1,1 \%)$. Entsprechend wird derzeit eine Wiederholung der Untersuchung bei unauffälligem Befund nach 5 Jahren empfohlen.

Da durch die Sigmoidoskopie isoliert proximal gelegene Tumoren nicht entdeckt werden können, ist weiterhin ein jährlicher FOBT sinnvoll. Dieser sollte vor einer Sigmoidoskopie durchgeführt werden, da bei positivem Test eine Koloskopie erforderlich ist und die Sigmoidoskopie entfallen kann. Der zusätzliche Nutzen der Kombination im Sinne einer Mortalitätssenkung ist jedoch nicht gesichert. Eine prospektive nicht randomisierte Studie fand zwar eine niedrigere KRK-bedingte Mortalität für die Kombination, das Ergebnis verfehlte jedoch grenzwertig die Signifikanz und die Compliance war ausgesprochen niedrig [126]. In mehreren Studien war jedoch eine Kombination aus Sigmoidoskopie und einmaligem FOBT der alleinigen Sigmoidoskopie nicht signifikant überlegen [127, 128]. In der aktuellsten Studie aus Japan wurden durch die Kombination aus Sigmoidoskopie und FIT absolut $10 \%$ mehr fortgeschrittene Neoplasien entdeckt [129].

$\mathrm{Zu}$ bedenken ist, dass die Sigmoidoskopie derzeit in Deutschland nicht im Leistungskatalog der Krankenkassen enthalten ist und somit nicht abgerechnet werden kann. Ferner sind derzeit anders als für die Vorsorgekoloskopie keine qualitätssichernden Maßnahmen zur Durchführung der Sigmoidoskopie etabliert. In England war eine Voraussetzung bei den Untersuchern für die Teilnahme an der Sigmoidoskopiestudie eine Mindestzahl von 50 angeleiteten und 100 eigenständigen Sigmoidoskopien [109]. Jede Untersuchung wurde per Video dokumentiert und die erreichte Eindringtiefe, die Qualität der Darmvorbereitung und die Ergebnisse dokumentiert. 


\subsubsection{Kapsel-Koloskopie}

\subsection{Empfehlung (2013)}

Die Kapsel-Koloskopie sollte nicht für die Darmkrebs-Vorsorge/ -Früherkennung in der asymptomatischen Bevölkerung eingesetzt werden.

Empfehlungsgrad B, Level of Evidence 4, de novo: [130-136], starker Konsens

\section{Hintergrund}

Zur Kapsel-Koloskopie konnte in der Literatursuche keine Studie zum Einsatz in der Früherkennung kolorektaler Karzinome identifiziert werden. Es existiert eine Reihe von Fallserien zur Sensitivität und Spezifität kolorektaler Neoplasien mit der ersten Kapselgeneration [130 - 134]. Für die zweite Kapselgeneration (PCC2) mit verbesserten technischen Eigenschaften wurden Sensitivitäten für Polypen größer $6 \mathrm{~mm}$ von $84-89 \%$ beschrieben $[135,136]$. Es handelt sich jedoch um kleine Kohorten mit vorselektionierten Patienten, sodass derzeit der Einsatz in der Früherkennung kolorektaler Karzinome in der Allgemeinbevölkerung nicht empfohlen werden kann.

\subsubsection{Stuhltests ${ }^{3}$}

4.2.2.1. Fäkaler okkulter Bluttest (FOBT)

4.6. Empfehlung/Statement (2013)

Bei Personen mit durchschnittlichem Darmkrebsrisiko, die keine Koloskopie wünschen, sollte ein FOBT jährlich durchgeführt werden. GCP, starker Konsens

\subsection{Statement (2008)}

Ein positives Testergebnis macht die endoskopische Untersuchung des gesamten Dickdarms erforderlich.

Level of Evidence 1a, starker Konsens

\subsection{Statement (2013)}

Der jährliche FOBT ist bezüglich einer Senkung der KRK-bedingten Mortalität der zweijährlichen Untersuchung überlegen.

Level of Evidence 1b, de novo: [137], starker Konsens

\subsection{Statement (2008)}

Bei Personen, die an der Koloskopie-Vorsorge/-Früherkennung teilnehmen, erübrigt sich ein FOBT und auch andere Maßnahmen. Level of Evidence 1a, starker Konsens

\subsection{Empfehlung (2013)}

Immunologische FOBT (iFOBT) mit nachgewiesen hoher Spezifität $>90 \%$ und Sensitivität können alternativ zum Guaiak-Test eingesetzt werden.

Empfehlungsgrad 0, Level of Evidence 2a, de novo: [102, 138 146], starker Konsens

\section{Hintergrund}

Grundlage für die Stuhltestung auf okkultes Blut ist die Tatsache, dass kolorektale Karzinome häufiger bluten als die normale Darmmukosa. Herkömmliche FOBT verwenden mit Guaiakharz imprägniertes Filterpapier, das sich in Anwesenheit von im Stuhl enthaltenem Hämoglobin nach Zugabe von Wasserstoffperoxid blau färbt. In Deutschland stehen aktuell drei Guaiaktests zur

\footnotetext{
${ }^{3}$ Prof. Schmiegel hat sich aufgrund eines möglichen Interessenkonflikts bei den Abstimmungen zum FOBT/iFOBT, genetischer Stuhltests und M2-PK enthalten (s. Leitlinienreport).
}

Verfügung: Hämoccult ${ }^{\circledR}$, HemoCare ${ }^{\circledR}$ und HemoFEC ${ }^{\circledR}$. Der sensitivste gFOBT, der Hämoccult Sensa ${ }^{\circledR}$ ist in Deutschland nicht verfügbar. Da viele Karzinome intermittierend bluten [147], führt die wiederholte Testung zu einer zuverlässigeren Erkennung von KRK [148, 149]. Das in den Studien eingesetzte Verfahren beinhaltet, aus drei aufeinander folgenden Stuhlgängen je zwei Proben pro Stuhl auf zwei Testfelder aufzutragen und auf okkultes Blut zu testen [150].

Zur Effektivität des FOBT als Früherkennungsmethode für kolorektale Karzinome liegen die Ergebnisse von 4 großen randomisierten Studien vor $[137,151,152]$. In der aktuellsten Meta-Analyse dieser Studien konnte eine Senkung der KRK-bedingten Mortalität um 25\% für diejenigen gezeigt werden, die sich mindestens einmal einem FOBT unterzogen hatten (relatives Risiko 0,75, $95 \%$ CI 0,66 - 0,84) [153]. In drei der vier Studien wurde der gFOBT alle zwei Jahre durchgeführt. In einer Studie mit ein- und zweijähriger Testung war die jährliche der zweijährigen Testung in Bezug auf die Reduktion der Mortalität überlegen [137].

Sensitivität und Spezifität des Tests hängen entscheidend von der Art der Testdurchführung und der Patienteninstruktion ab. Eine Rehydrierung der Testbriefchen vor Entwicklung steigert die Sensitivität des Screenings, verringert jedoch die Spezifität deutlich (in einer Studie von 97,6 auf 90,2\%, in einer weiteren von 97 auf $85,4 \%[137,154])$ und wird daher nicht empfohlen. Es gibt Hinweise dafür, dass die Instruktion des Patienten vor der Testdurchführung in Bezug auf Ernährung und interferierende Medikamente die Zahl der falsch-positiven Testergebnisse und somit auch die Zahl der erforderlichen Koloskopien reduzieren kann [155 - 157]. Es erscheint daher sinnvoll, den Patienten über Faktoren, die das Testergebnis beeinflussen könnten, aufzuklären. Der Einfluss von Pflanzenperoxidasen kann alternativ durch eine Testentwicklung 3 Tage nach Durchführung vermieden werden [158]. Die Notwendigkeit einer Ernährungsempfehlung für den Hämoccult ${ }^{\circledR}$ wird allerdings durch eine Metaanalyse infrage gestellt [159].

Bereits bei positivem Testergebnis auf okkultes fäkales Blut von einem der Testfelder ist keine Kontrolle, sondern eine komplette endoskopische Darstellung des Dickdarms nach digitaler rektaler Untersuchung erforderlich. Leider wurden selbst unter Studienbedingungen teilweise unter $90 \%$ aller Personen mit einem positivem FOBT koloskopiert [160], in einer Studie sogar nur 64\% [161].

Der Effekt des FOBT beruht auf einer Diagnose kolorektaler Karzinome in einem früheren prognosegünstigeren Stadium. Vorteile des FOBT sind die leichte Durchführbarkeit sowie die geringen Kosten. Nachteilig ist eine mäßige Sensitivität für Karzinome und eine geringe Sensitivität für Adenome. In einer randomisierten Studie konnte zwar eine Senkung der Inzidenz kolorektaler Karzinome gezeigt werden, es muss jedoch bedacht werden, dass im Rahmen dieser Studie über 30\% der Teilnehmer koloskopiert wurden [162].

Immunologische Tests weisen spezifisch menschliches Hämoglobin nach. Insofern ist während der Testdurchführung keine Änderung der Ernährung erforderlich. Auch besteht anders als bei den gFOBT bei einigen der Tests die Möglichkeit einer automatisierten Auswertung und Änderung des Hämoglobingrenzwerts, bei dem ein Test als positiv bewertet wird. Die Tests werden entweder als immunologische FOBT (iFOBT) oder als fäkale immonochemische Tests (FIT) bezeichnet und stellen derzeit in Deutschland keine Kassenleistung dar. Es existieren keine Studien wie für den gFOBT, die eine Senkung der KRK-bedingten Mortalität zum Endpunkt hätten. Jedoch gibt es eine Reihe randomi- 
sierter Studien, in denen einzelne iFOBT direkt mit gewissen gFOBT verglichen werden. In der im Rahmen der Literatursuche identifizierten Metaanalyse dieser Studien waren einzelne iFOBT (OC-Hemodia ${ }^{\circledR}$ ) dem Hämoccult ${ }^{\circledR}$-Test in der Detektionsrate fortgeschrittener Neoplasien signifikant überlegen mit einer pooled odds ratio (OR) von 2,12 (95\% CI 1,66 - 2,71) [138]. In zwei Studien, in denen der gFOBT HemoFEC ${ }^{\circledR}$ bzw. Hämoccult Sensa ${ }^{\circledR}$ mit einem iFOBT (Inform ${ }^{\circledR}$ bzw. FlexSure ${ }^{\circledR}$ ) verglichen wurde, fand sich hingegen kein signifikanter Unterschied [138]. Insbesondere in den beiden größten randomisierten Studien aus den Niederlanden $[102,139]$ fand sich jedoch ein signifikanter Unterschied, die für eine Überlegenheit des eingesetzten iFOBT (OC Hemodia) gegenüber dem Hämoccult ${ }^{\circledR}$-Test sprechen.

Die Sensitivität und Spezifität von in Deutschland verfügbaren iFOBT variiert deutlich, sodass der generelle Einsatz aller iFOBT nicht empfohlen werden kann [140]. Es erscheint vielmehr erforderlich, dass für jeden iFOBT, der für die Krebsfrüherkennung eingesetzt werden soll, individuell eine ausreichend hohe Sensitivität und vor allem Spezifität nachgewiesen wird. Für die Spezifität wird allgemein die untere akzeptable Grenze bei $90 \%$ angesehen. Die Ergebnisse von Screening-Studien legen nahe, dass, bei entsprechender Einstellung des Grenzwerts, mit den iFOBTs eine dem gFOBT vergleichbar hohe Spezifität von $>90 \%$ bei zugleich sehr viel höherer Sensitivität erreicht werden kann [141, 142]. Diskutiert wird derzeit noch der optimale HämoglobinGehalt, bei dem ein iFOBT als positiv bewertet werden sollte. In den beiden niederländischen Arbeiten betrug der Grenzwert $100 \mathrm{ng} / \mathrm{ml}$. In den eingeschlossenen Studien wurde jeweils eine Stuhlprobe mittels des iFOBT untersucht. Es gibt Daten die zeigen, dass die Testung von mehreren Stuhlproben die Sensitivität erhöht [143-145]. Eine Untersuchung aus den Niederlanden konnte jedoch zeigen, dass durch eine Absenkung des Grenzwerts ein ähnlicher Effekt erzielt werden kann [146].

Insgesamt zeigt die Datenlage, dass die iFOBT, für die eine entsprechende Datengrundlage vorhanden ist, einen sinnvollen Ersatz für den gFOBT darstellen.

\subsubsection{Genetische Stuhltests}

\subsection{Empfehlung (2013)}

Stuhluntersuchungen auf DNA-Veränderungen sollten nicht für die Darmkrebs-Vorsorge/-Früherkennung in der asymptomatischen Bevölkerung eingesetzt werden.

Empfehlungsgrad B, Level of Evidence 2b, de novo: [163-168], starker Konsens

\section{Hintergrund}

Die Entstehung kolorektaler Karzinome über die Zwischenstufe der Adenome geht in vielen Fällen mit charakteristischen genetischen Veränderungen einher. Eine Isolierung und Untersuchung von DNA aus Kolonepithelzellen im Stuhl ist möglich [163 - 166]. Dieses Testverfahren hat den Vorteil, dass theoretisch auch nicht blutende Läsionen entdeckt werden könnten. In einer Studie von Imperiale mit nahezu 5500 asymptomatischen Personen wurde eine Stuhlprobe von 2500 Teilnehmern auf insgesamt 23 genetische Veränderungen untersucht und mit dem gFOBT verglichen [167]. Die Sensitivität des genetischen Tests war höher als der des gFOBT, betrug aber lediglich $50 \%$ für Karzinome und 15\% für fortgeschrittene Adenome bei Kosten von mehreren Hundert US Dollar pro Test und aufwändiger Testdurchführung. In der Literatursuche wurde lediglich eine weitere Studie identifiziert [168]. In dieser prospektiven Studie wurden bei 3764 asympto- matischen Personen zwischen 50 und 80 Jahren ein gFOBT sowie eine Koloskopie durchgeführt. Von 2497 dieser Teilnehmer wurde eine Stuhlprobe mit einem DNA-Panel I mit denselben Markern wie in der Studie von Imperiale und von 217 mit einem DNA-Panel II mit nur noch 3 Mutationen inklusive Methylierungsmarker Vimentin untersucht. Die Sensitivität für relevante Neoplasien betrug $20 \%$ für DNA-Panel I und $40 \%$ für DNA-Panel II. Die Sensitivität für den Hämoccult ${ }^{\circledR}$-Test betrug $11 \%$, für den Hämoccult-Sensa $^{\circledR} 21 \%$. Die Spezifität für das DNA-Panel II konnte nicht ermittelt werden. Insgesamt war Panel I dem einen gFOBT (Hämoccult ${ }^{\circledR}$ ) überlegen und dem anderen gFOBT (HämoccultSensa ${ }^{\circledR}$ ) gleichwertig. Panel II schien beiden gFOBT überlegen, wurde jedoch nur bei einem kleinen Teil der Teilnehmer untersucht.

Zusammenfassend sind die Daten bzw. Ergebnisse unter Berücksichtigung des Aufwands und der Kosten für einzelne Tests nicht ausreichend, sodass der Einsatz aktuell nicht erfolgen sollte.

\subsubsection{M2-PK}

\subsection{Empfehlung (2013)}

Der M2-PK-Stuhltest sollte nicht für die Darmkrebs-Vorsorge/Früherkennung in der asymptomatischen Bevölkerung eingesetzt werden.

Empfehlungsgrad B, Level of Evidence 3b, de novo: [169, 170], starker Konsens

\section{Hintergrund}

Die Mehrzahl der Studien erfolgte an vorselektierten Patientenkollektiven und erlaubt somit keine Aussage zur Wertigkeit des Tests für die Vorsorge/Früherkennung in der asymptomatischen Bevölkerung. In der extern durchgeführten Literatursuche wurden zwei Studien $[169,170]$ zur Untersuchung einer Vorsorge-/ Früherkennungspopulation identifiziert. In einer Studie wurde der M2-PK-Test mit der Koloskopie bei 1082 asymptomatischen Personen verglichen. Die Sensitivität für fortgeschrittene Adenome betrug $21,7 \%$ bei einer Spezifität von $82 \%$. In einer weiteren Studie mit 1079 Teilnehmern wurde der M2-PK-Test mit verschiedenen FOBT verglichen. Die Sensitivität für fortgeschrittene Neoplasien betrug 27,3\% (im Vergleich zu 7,3 - 20\% für die FOBT) bei einer Spezifität von 86,2\% (FOBT 92,9-94,0\%). Der positive prädiktive Wert für fortgeschrittene Neoplasien betrug 11,5\% und war niedriger als alle getesteten FOBT. Insgesamt ist die Datenlage nicht ausreichend, um den Test für die Vorsorge/Früherkennung in der asymptomatischen Bevölkerung empfehlen zu können.

\subsubsection{Radiologische Verfahren}

\subsection{Empfehlung (2013)}

Die CT-Kolonografie und die MR-Kolonografie sollten nicht für die Darmkrebs-Vorsorge/-Früherkennung in der asymptomatischen Bevölkerung eingesetzt werden.

Bei inkompletter Koloskopie (z. B. Adhäsionen) und fortbestehendem Wunsch des Patienten auf komplette Kolonbeurteilung sollte eine CT- oder MR-Kolonografie erfolgen.

Empfehlungsgrad B, Level of Evidence 3b, de novo: [171-173], starker Konsens

\section{Hintergrund}

Für den Einsatz der MR-Kolonografie existieren nur einzelne kleine Studien, sodass die Datenlage nicht ausreicht, um einen Einsatz für die Vorsorge/Früherkennung zu befürworten. Für die 
CT-Kolonografie (CTC) ist die Datenlage umfangreicher. In den beiden in der Literatursuche identifizierten aktuellsten Metaanalysen für den Vergleich der CTC und der Koloskopie als VorsorgeFrüherkennungsmaßnahme in der asymptomatischen Bevölkerung zeigte sich eine hohe Sensitivität von $100 \%$ für die Detektion von Karzinomen und von 87,9\% für die Detektion von Adenomen $\geq 10 \mathrm{~mm}$. Die Sensitivität für kleinere Adenome war geringer [171, 172]. Weiterhin fällt zwischen den verschiedenen Studien eine nicht unerhebliche Heterogenität auf. Es bleibt unklar, ob die Ergebnisse der Studien, die an erfahrenen Zentren durchgeführt wurden, auf die klinische Praxis übertragbar sind. Weiterhin ist die Bedeutung extrakolonischer Befunde unklar. Die Untersuchung geht mit einer Strahlenexposition einher, sodass sich der Einsatz bei vorhandenen Alternativmethoden in Deutschland als Früherkennungs-/Vorsorgemethode aufgrund der Strahlenschutzverordnung verbietet (StrlSchV §80). Das genaue Neoplasierisiko durch eine CTC unter Verwendung neuerer Geräte mit verringerter Strahlendosis ist unklar. Unklar ist weiterhin, ab welcher Polypengröße eine Koloskopie durchgeführt werden sollte und in welchem Intervall Patienten mit unauffälliger CTC oder kleineren Polypen kontrolliert werden sollten [173].

Patienten, bei denen eine Koloskopie aus technischen Gründen nur inkomplett durchgeführt werden konnte, sollte eine Wiederholung der Koloskopie z. B. unter stationären Bedingungen oder die CTC als Alternative zur Beurteilung des restlichen Kolons angeboten werden (s. Kapitel 6.1.)

\subsection{Kosteneffektivität}

4.14. Statement (2013)

Sowohl FOBT als auch Sigmoidoskopie, Koloskopie und die Kombination aus Sigmoidoskopie und FOBT gelten als kosteneffektiv (im Vergleich zu Vorsorge-/Früherkennungsverfahren anderer Zielkrankheiten).

GCP, Konsens

\section{Hintergrund}

Prospektive Studien zur Kosteneffektivität der verschiedenen KRK-Vorsorgeverfahren existieren nicht. Mathematische Modellrechnungen legen nahe, dass Koloskopie, Sigmoidoskopie und FOBT kosteneffektiv sind [139, $174-184]$.

\subsection{Empfehlungen anderer eingeschlossener Leitlinien}

Anhand der DELBI-Kriterien wurden 2 Leitlinien zur KRK-Vorsorge/-Früherkennung eingeschlossen. Zum einen die Empfehlungen der US Preventive Task Force aus dem Jahr 2008 [94] und zum anderen die Asian Pacific consensus recommendations aus dem Jahr 2007 [185]. Von der US Preventive Task Force werden die folgenden Methoden empfohlen: ein sensitiver FOBT (aufgeführt Hämoccult sensa ${ }^{\circledR}$ und IFOBT/FIT) jährlich, eine Sigmoidoskopie alle 5 Jahre zusammen mit einem FOBT alle 3 Jahre oder eine Koloskopie alle 10 Jahre. Der Einsatz der CT-Kolonografie und genetischer Stuhltests wird nicht empfohlen, die Kapselendoskopie und der M2-PK-Test werden nicht aufgeführt. Zu erwähnen ist, dass der Hämoccult sensa ${ }^{\circledR}$ in Deutschland nicht verfügbar ist.

In der Asian-Pacific-Leitlinie werden der FOBT (gFOBT und iFOBT) alle 1 - 2 Jahre, die Sigmoidoskopie alle 5 Jahre und die Koloskopie alle 10 Jahre empfohlen. Die CT-Kolonografie wird nicht empfohlen, genetische Stuhltests, Kapselendoskopie und M2-PK-Test sind nicht aufgeführt.

\section{Risikogruppen}

$\nabla$

Personen, die aufgrund einer besonderen Prädisposition ein erhöhtes Risiko für die Entwicklung eines kolorektalen Karzinoms im Vergleich mit der Normalbevölkerung aufweisen, gehören in der Regel zu einer von drei definierten Risikogruppen:

- Personen mit einem familiär gesteigerten Risiko für ein kolorektales Karzinom, dessen genetische Grundlagen zzt. noch nicht umfassend bekannt sind

- nachgewiesene oder mögliche Anlageträger für ein hereditäres kolorektales Karzinom

- Risikopersonen auf dem Boden einer chronisch entzündlichen Darmerkrankung

\subsection{Sporadisches kolorektales Karzinom}

5.1.1. Risikogruppen

5.1.1.1. Verwandte von Patienten mit kolorektalem Karzinom

5.1. Statement (2013)

Verwandte ersten Grades von Patienten mit einem kolorektalen Karzinom haben ein erhöhtes Risiko, ebenfalls an einem kolorektalen Karzinom zu erkranken.

Level of Evidence 2a, Evidenz aus Aktualisierungsrecherche: [186 - 200], Konsens

\subsection{Statement (2013)}

Verwandte zweiten Grades haben ein gering erhöhtes Risiko, an einem kolorektalen Karzinom zu erkranken.

Level of Evidence 2b, Evidenz aus Aktualisierungsrecherche: [186, 187, 190, 191, 201, 202], Konsens

\section{Hintergrund}

Etwa 20 - 30\% der KRK-Fälle treten „familiär gehäuft“ auf, d. h. es kann ein gehäuftes Vorkommen der Krebserkrankung innerhalb einer Familie beobachtet werden, ohne dass jedoch konkrete genetische Ursachen identifiziert werden können. In diesem Fall wird der Begriff „Familiäres kolorektales Karzinom“ verwendet [186]. Für Verwandte ersten Grades (Eltern, Geschwister, Kinder) eines Patienten mit einem KRK ist das mittlere Risiko 2- bis 3fach erhöht. Eine weitere, 3- bis 4-fache Risikosteigerung besteht, wenn bei dem Indexpatienten das KRK vor dem 60. Lebensjahr aufgetreten ist und/oder mehr als ein Verwandter ersten Grades von einem KRK betroffen ist [187 - 199]. In dieser Gruppe befinden sich allerdings auch bislang unentdeckte hereditäre KRK (z.B. HNPCC; s. u.). Das Risiko ist für das Kolon- im Vergleich zum Rektumkarzinom höher (relatives Risiko 2,4 vs. 1,9). Für erstgradig Verwandte von betroffenen Patienten kann das KRKRisiko weiter aufgeteilt werden. So ist das Risiko für die Geschwister etwa 2,5-fach höher als für die Kinder. Ist der Indexpatient nach dem 60. Lebensjahr erkrankt, ist das KRK-Risiko für die erstgradig Verwandten nur noch gering erhöht [188, 200]. Verwandte zweiten Grades (Großeltern, Geschwister der Eltern, Enkel) von Patienten mit kolorektalen Karzinomen haben ein leicht erhöhtes Karzinomrisiko (RR 1,5); dieses ist aber derzeit nur unzureichend untersucht und bisher nicht in der Praxis verifiziert [187, 190, 191, 201, 202]. Für Verwandte dritten Grades von Patienten mit KRK ist kein erhöhtes Karzinomrisiko anzunehmen. 


\subsubsection{Verwandte von Patienten mit kolorektalem Adenom}

\subsection{Statement (2013)}

Verwandte ersten Grades von Patienten, bei denen ein kolorektales Adenom vor dem 50. Lebensjahr nachgewiesen wurde, haben ein erhöhtes Risiko, an einem kolorektalen Karzinom zu erkranken. Level of Evidence 2b, Evidenz aus Aktualisierungsrecherche: [188, 191, 203 - 206], Konsens

\section{Hintergrund}

Das Risiko dieser Verwandten, ein KRK zu entwickeln, ist im Mittel etwa 2-fach gegenüber der Allgemeinbevölkerung gesteigert [188, 191, 203 -206]; es besteht ein 80\% höheres Risiko bei Eltern und Geschwistern von Adenom-Patienten im Vergleich mit deren Lebenspartnern [203]. Auch hier ist die Risikohöhe vom Alter des Indexpatienten abhängig: Ist dieser jünger als 60 Jahre, ist das mittlere Risiko nur leicht erhöht, ist er jünger als 50 Jahre, ist das Risiko ca. 4,4-fach erhöht [204]. Ist der Indexpatient älter als 60 Jahre, ist das kolorektale Karzinomrisiko nicht mehr statistisch signifikant erhöht.

Aufgrund der Datenlage gibt es keine Evidenz, dass Verwandte von Patienten, bei denen ein hyperplastischer Polyp nachgewiesen wurde, ein erhöhtes Risiko haben, an einem kolorektalen Karzinom zu erkranken. Eine Ausnahme ist das sehr seltene hyperplastische Polyposis-Syndrom.

\subsubsection{Patienten mit kolorektalen Adenomen}

\subsection{Statement (2008)}

Jedes histologisch nachgewiesene Adenom stellt ein erhöhtes Risiko für ein kolorektales Karzinom dar. Dies gilt insbesondere für:

- multiple $(\geq 3)$ Adenome

- $\operatorname{große}(>1 \mathrm{~cm})$ Adenome

Level of Evidence 2b, [99, 100, 207, 208], Konsens

\section{Hintergrund}

Generell führt die Abtragung kleiner, singulärer Adenome im Vergleich zur Normalbevölkerung zu einem um bis zu $90 \%$ verminderten Risiko, ein metachrones KRK zu entwickeln [99, 100, 207, 208]. Dieses reflektiert den Vorsorgewert der Koloskopie im Rahmen der Adenom-Karzinom-Sequenz. Kontrolluntersuchungen dienen insbesondere der Entdeckung übersehener oder metachron aufgetretener Adenome.

Adenome $>1 \mathrm{~cm}$ sind mit einem etwa 4-fach erhöhten Karzinomrisiko assoziiert [191, 208-215]. Auch bei multiplen Adenomen ist das Risiko, ein metachrones Karzinom zu entwickeln, deutlich (4bis 6-fach) gesteigert [191, 208, 210, 211, 213, 214]. Hierbei dürfte das erhöhte Risiko einerseits auf einer stärkeren individuellen Disposition, andererseits auf einer höheren Prävalenz übersehener Polypen bei der initialen Koloskopie beruhen: beim koloskopischen Nachweis von $\geq 3$ Polypen besteht eine signifikant größere Wahrscheinlichkeit, dass weitere Polypen übersehen wurden $[117,216]$.

\subsection{Konsensbasierte Empfehlung (2013)}

Ein erhöhtes familiäres Darmkrebsrisiko wird durch die ärztliche Anamnese erfasst. Jedoch besteht keine verpflichtende Erfassung der Familienanamnese und ggf. deren Wiederholung im Intervall. Die Verwendung von standardisierten Fragebogen kann geeignet sein, die Erfassung von Personen mit erhöhtem Risiko zu verbessern. GCP, starker Konsens

\section{Hintergrund}

Die Risikofeststellung erfolgt in der Regel über das ärztliche Anamnesegespräch. Eine Möglichkeit zur Integration der Risiko- feststellung in die medizinische Betreuung bestünde in der Verwendung eines standardisierten Fragebogens in der hausärztlichen Praxis im Alter von 35 Jahren im Rahmen der zu diesem Zeitpunkt angebotenen Check-up-Untersuchung.

Durch eine Integration in die Praxis-Software als Memo kann diese Befragung nachgeholt werden, sofern der Patient zu dem o.g. Zeitpunkt nicht in der Praxis vorstellig wird. Ein entsprechendes Konzept wird gegenwärtig im Gemeinsamen Bundesausschuss geprüft.

Weitere sinnvolle Stellen sind gynäkologische Praxen, da hier regelmäßige Vorstellungen im Rahmen der gynäkologischen Krebsfrüherkennung empfohlen werden, sowie Vorstellungen in gastroenterologischen Praxen.

Darüber hinaus steht eine Reihe von Fragebogen, die teilweise im Internet frei zugänglich sind, zur Verfügung:

- http://www.onkozert.de/hinweise_zertifizierung_genetische_beratung.htm

- http://www.netzwerk-gegen-darmkrebs.de/index.php? id $=103$

- http://www.felix-burda-stiftung.de/darmkrebs/frageboegen/ index.php? $\mathrm{f}=2$

- http://www.lebensblicke.de/darmkrebs/http://www.ilco.de/ darmkrebs/erblicher-darmkrebs.html

- http://www.krebsrisikotest.de/

Von den genannten Fragebogen ist der vom „Netzwerk gegen Darmkrebs“ verwendete Fragebogen evaluiert worden [217]. Zu den anderen verwendeten Fragebögen liegen bisher keine Daten vor.

\subsubsection{Primärprävention}

\subsection{Statement (2013)}

Eine gesonderte Empfehlung zur Primärprävention (diätetische Maßnahmen, Chemoprävention) im Vergleich zur Normalbevölkerung kann aufgrund der widersprüchlichen Daten für die genannten Risikogruppen nicht gegeben werden.

Level of Evidence 1b, Evidenz aus Aktualisierungsrecherche: [218 - 221], starker Konsens

\section{Hintergrund}

Generell können die für die Normalpopulation genannten Empfehlungen (s. Kapitel 3) auch für die Angehörigen der Risikogruppen übernommen werden; für spezielle Maßnahmen fehlen gesicherte Daten [218-221].

\subsubsection{Vorsorgeuntersuchungen}

5.1.3.1. Verwandte ersten Grades von Patienten mit kolorektalem Karzinom

\subsection{Konsensbasierte Empfehlung (2013)}

Verwandte ersten Grades von Patienten mit kolorektalem Karzinom sollten in einem Lebensalter, das 10 Jahre vor dem Alterszeitpunkt des Auftretens des Karzinoms beim Indexpatienten liegt, erstmals komplett koloskopiert werden, spätestens im Alter von 40-45 Jahren. Die Koloskopie sollte bei polypenfreiem Darm in der initialen Koloskopie mindestens alle 10 Jahre wiederholt werden.

GCP, starker Konsens

\section{Hintergrund}

Das Risiko eines Verwandten ersten Grades eines Patienten mit kolorektalem Karzinom, ebenfalls an einem kolorektalen Karzinom zu erkranken, ist, insbesondere bei einem Manifestationsalter unter 50 Jahren beim Indexpatienten, erhöht [191, 193, 200, 202, 222 - 227]. 
Die Empfehlung lehnt sich an die amerikanische Leitlinienempfehlung an [228]. Dort wird eine Empfehlung zur Koloskopie im Alter von 40 Jahren ausgesprochen, wenn ein erstgradig Verwandter vor dem 60. Lebensjahr an einem KRK erkrankt ist oder zwei oder mehr erstgradig Verwandte an einem KRK (unabhängig vom Erkrankungsalter) erkrankt sind.

Die Frage des maximalen Untersuchungsintervalls ist bisher nicht eindeutig geklärt; es gilt aber als wahrscheinlich, dass ein Intervall von 10 Jahren in der Regel ausreichen dürfte, dieses aber nicht überschritten werden sollte. In der amerikanischen Leitlinie wird ein 5-Jahres-Intervall empfohlen.

\subsection{Konsensbasierte Empfehlung (2013)}

Erstgradig Verwandte von Patienten aus Familien, die die Amsterdam-Kriterien erfüllen und gleichzeitig eine Mikrosatellitenstabilität (MSS) im Karzinom aufweisen, sollten engmaschiger überwacht werden:

Zeigen mindestens zwei unabhängige Karzinome aus der Familie eine MSS, sollte ab dem 25. Lebensjahr in Intervallen von 3-5 Jahren koloskopiert werden.

Ist nur ein Karzinom aus der Familie untersucht worden und weist eine MSS auf, sollten zusätzlich Früherkennungsuntersuchungen auf Endometriumkarzinom und Magenkarzinom ebenfalls in Intervallen von 3-5 Jahren durchgeführt werden.

GCP, starker Konsens

\subsection{Konsensbasierte Empfehlung (2013)}

Bei erstgradig Verwandten von Patienten mit kolorektalem Karzinom aus Familien, in denen die Bethesda-Kriterien, nicht aber die Amsterdam-Kriterien erfüllt sind, sollte in kürzeren Intervallen koloskopiert werden:

Wenn kein Tumorgewebe zur Untersuchung auf HNPCC-typische Auffälligkeiten zur Verfügung steht, sollte das Intervall 3 Jahre nicht überschreiten.

GCP, Konsens

\subsection{Empfehlung (2013)}

Wenn das Tumorgewebe eine Mikrosatellitenstabilität (MSS) oder eine niedriggradige Mikrosatelliteninstabilität (MSI-L) zeigt, sollte das Intervall 3 - 5 Jahre betragen.

GCP, Konsens

\section{Hintergrund}

Bei jungen Indexpatienten in der Verwandtschaft sollte die Diagnose eines HNPCC-Syndroms (s. Abschnitt 5.2.1) in Erwägung gezogen werden und eine Mikrosatellitenanalyse und/oder immunhistochemische Untersuchung der Mismatch-ReparaturProteine durchgeführt werden. In der klinischen Praxis finden sich immer wieder Familien, in denen eine Häufung kolorektaler Karzinome vorliegt, jedoch ohne, dass die klinischen Diagnosekriterien für HNPCC (Amsterdam-II-Kriterien) erfüllt sind. Sollte in diesen Familien kein Tumorgewebe mehr verfügbar sein oder dieses eine Mikrosatellitenstabilität (MSS) zeigen, kann das Vorliegen einer bislang unbekannten erblich bedingten gastrointestinalen Tumordisposition nicht sicher ausgeschlossen werden. Ein Untersuchungsintervall von höchstens 10 Jahren erscheint in dieser Konstellation nicht engmaschig genug, auch wenn diese Fragestellung bisher nicht eindeutig geklärt ist; ein Intervall von 3 bis 5 Jahren dürfte dem familiären Risiko angemessen sein. Bei Patienten aus Familien, welche die Amsterdam-Kriterien erfüllen, eine Mikrosatelliteninstabilität aber ausgeschlossen werden konnte, wurde bisher analog zu den Familien mit nachgewie- sener MSI eine jährliche Vorsorge empfohlen. Dies ist aufgrund der Datenlage nicht erforderlich. Intervalle von 3 bis 5 Jahren sind angemessen [229, 230]. Eine Überwachung hinsichtlich extrakolonischer Tumoren ist bei diesem Personenkreis außerhalb der allgemeinen Krebsfrüherkennungsmaßnahmen nicht erforderlich.

\subsubsection{Verwandte von Patienten mit kolorektalen Adenomen}

\subsection{Konsensbasierte Empfehlung (2013)}

Verwandte ersten Grades von Indexpatienten, bei denen Adenome vor dem 50. Lebensjahr nachgewiesen wurden, sollten 10 Jahre vor dem Lebensalter zum Zeitpunkt des Nachweises des Adenoms koloskopiert werden. Die Koloskopie sollte bei polypenfreiem Darm in der initialen Koloskopie mindestens alle 10 Jahre wiederholt werden. Bei Nachweis von Polypen gelten die Empfehlungen von Kapitel 6.5. GCP, starker Konsens

\section{Hintergrund}

Die Empfehlung basiert auf dem in Abschnitt 5.1.1 dargelegten erhöhten Risiko dieser Population [92, 193, 202, 204].

\subsection{Hereditäre kolorektale Karzinome}

\subsection{Konsensbasierte Empfehlung (2013)}

Wenn Grund zu der Annahme besteht, dass bei einem Patienten eine erbliche Form des Darmkrebses vorliegt oder, dass eine gesunde Person ein hohes Risiko für eine erbliche Form von Darmkrebs hat, sollte der Patient in einem interdisziplinären Zentrum mit ausgewiesener Expertise auf dem Gebiet des erblichen Darmkrebses vorgestellt werden.

GCP, Konsens

\section{Hintergrund}

Monogen erbliche kolorektale Karzinome sind selten (weniger als $5 \%$ aller kolorektalen Karzinome). Die Diagnose hat erhebliche Konsequenzen für die Patienten und ihre Verwandten. Im Verdachtsfall sollte ein erfahrenes Zentrum für familiären Darmkrebs oder ein äquivalentes Angebot mit entsprechender Expertise kontaktiert werden. Eine molekulargenetische Diagnostik bei erkrankten Patienten dient der Diagnosesicherung und ermöglicht die prädiktive Testung von Familienangehörigen. Eine entsprechende Keimbahnmutationssuche soll nach den Richtlinien zur Diagnostik der genetischen Disposition für Krebserkrankungen der Bundesärztekammer und dem Gendiagnostikgesetz (GenDG) erfolgen [120]. Beispielhaft ist der Algorithmus für das HNPCC/Lynch-Syndrom in $\bullet$ Abb. 2 dargestellt. Er gilt in analoger Weise für die anderen erblichen Syndrome mit erhöhtem Darmkrebsrisiko. Neben einem erhöhten Risiko für kolorektale Karzinome haben alle Patienten und Risikopersonen dieser Gruppe ein zusätzlich erhöhtes Risiko extrakolischer Neoplasien. Aufgrund des meist autosomal-dominanten Erbgangs haben erstgradig Verwandte von Betroffenen ein 50\%iges Risiko, diese genetische Disposition ebenfalls geerbt zu haben. Eine prädiktive genetische Testung bei diesen gesunden Risikopersonen muss dem GenDG entsprechend an eine genetische Beratung gebunden sein und kann in der Regel nur erfolgen, wenn eine zweifelsfrei pathogene Keimbahnmutation bei einem erkrankten Familienmitglied nachgewiesen wurde ( $\bullet$ Abb. 2) [120].

\subsection{Konsensbasierte Empfehlung (2013)}

Bereits erkrankte Personen, Anlageträger und Risikopersonen für monogen erbliche Erkrankungen mit erhöhtem Risiko für kolorek- 
tale Karzinome sollen auf Möglichkeit und Nutzen einer psychosozialen Beratung und Betreuung hingewiesen werden.

GCP, starker Konsens

\section{Hintergrund}

Die Diagnose eines manifesten erblichen Tumorsyndroms, das Wissen um ein deutlich erhöhtes Erkrankungsrisiko oder der definitive Nachweis der Anlageträgerschaft können bei den Betroffenen und ihren Angehörigen mit einer Vielzahl psychosozialer Stressfaktoren einhergehen. Entsprechende Untersuchungen wurden insbesondere bei der FAP durchgeführt und schlossen neben Erwachsenen und Jugendlichen auch Kinder und deren Eltern ein [231 - 233]. Zu den relevante Stressoren gehören eine veränderte Körperwahrnehmung, Ängste in Bezug auf chirurgische Eingriffe, Früherkennungsuntersuchungen und eine zukünftige Krebsentwicklung, befürchtete berufliche Einschränkungen, das Kommunizieren des Krankheitsbilds im sozialen Umfeld, Unsicherheit in Bezug auf reproduktive Entscheidungen sowie das Verarbeiten früh an Krebs verstorbener Familienangehöriger und damit zusammenhängender innerfamiliärer Konflikte.

Die prädiktive Testung Minderjähriger geht zusätzlich mit spezifischen Herausforderungen wie fehlender eigener Entscheidungsfähigkeit und eingeschränktem Verständnis von Sinn und Konsequenzen der Testung einher. Ergänzend zur klinischen und humangenetischen Beratung kann die psychosoziale Beratung die Patienten und Risikopersonen im Prozess der Entscheidungsfindung für bzw. gegen eine prädiktive genetische Diagnostik unterstützen und für die Verarbeitung von Testergebnissen hilfreich sein.

\subsubsection{Risikogruppen}

5.2.1.1. HNPCC(hereditäres kolorektales Karzinom ohne Polyposis)-/Lynch-Syndrom

\subsection{Statement (2013)}

Personen aus Familien, die die Amsterdam-Kriterien erfüllen oder eines der Bethesda-Kriterien mit Nachweis einer Mikrosatelliteninstabilität (MSI) und deren Verwandte, die aufgrund des Erbgangs als Mutationsträger in Betracht kommen, sind Risikopersonen für HNPCC.

GCP, starker Konsens

\section{Hintergrund}

Das HNPCC-Syndrom wird nach anamnestischen Kriterien definiert (Amsterdam-I- und -II-Kriterien, s. Kapitel 12.2). Um weitere Risikopersonen zu identifizieren, werden auch die revidierten Bethesda-Kriterien herangezogen (Bethesda-Kriterien, s. Kapitel 12.3). In der Literatur wird favorisiert, Anlageträger einer pathogenen Keimbahnmutation in einem der MMR-Gene als Personen mit Lynch-Syndrom zu bezeichnen. Demgegenüber konnte bei Patienten mit einem HNPCC keine pathogene Keimbahnmutation nachgewiesen werden. Zur Vereinfachung wird nachfolgend nur der Begriff HNPCC verwendet.

Mutationsträger haben ein sehr hohes Risiko, ein kolorektales Karzinom (50 - 70\%) oder ein Endometriumkarzinom (20 - 60\%) zu entwickeln. Dies gilt vermindert auch für weitere Neoplasien wie Ovarial-, Magen- und Dünndarmkarzinome sowie Urothelkarzinome des Nierenbeckens und Harnleiters.

\subsection{Konsensbasierte Empfehlung (2013)}

Die Feststellung, ob bei einem Patienten die Bethesda- oder Amsterdam-Kriterien für HNPCC erfüllt sind, soll anamnestisch durch die behandelnden Ärzte erfolgen.

GCP, starker Konsens

\section{Hintergrund}

Im Gegensatz zur FAP ist HNPCC aufgrund eines fehlenden auffälligen Phänotyps klinisch nicht einfach zu identifizieren. Aus diesem Grunde wurden Kriterien definiert, die es sinnvoll erscheinen lassen, zunächst eine Untersuchung des Tumorgewebes und anschließend ggf. eine Mutationssuche durchzuführen. Die Verdachtsdiagnose HNPCC kann klinisch gestellt werden, wenn in der Familie des Patienten die so genannten Amsterdam-I-Kriterien erfüllt sind [234]. Beim HNPCC treten neben KRK häufig auch Endometrium- und Urothelkarzinome aber auch Dünndarmkarzinome auf. Die Amsterdam-II-Kriterien beziehen diese extrakolischen Manifestationen in die Diagnosestellung ein [179]. Diese Kriterien stellen unter klinischen Gesichtspunkten eine Operationalisierung dar. Da viele Familien heute klein sind, fehlen allerdings oft die Voraussetzungen, um die Kriterien überhaupt zu erfüllen. Daher kann eine unauffällige Familienanamnese - insbesondere in kleinen Familien - kein Argument gegen HNPCC sein. Die weniger spezifischen Bethesda-Kriterien begründen den Verdacht HNPCC auch in kleineren Familien und in Einzelfällen (Anlage 5.3) [235].

Das Risiko von HNPCC-Anlageträgern, mindestens ein HNPCC-assoziiertes Karzinom zu entwickeln, wird mit 80 - 90\% angegeben, wobei das KRK die mit Abstand häufigste Tumorentität darstellt. KRK treten im Rahmen eines HNPCC im Mittel im 44. Lebensjahr auf, vor dem 25. Lebensjahr sind kolorektale Karzinome bei HNPCC sehr selten. Das kumulative Lebenszeitrisiko eines HNPCC-Anlageträgers für ein KRK beträgt ca. 60 - 70\%, wobei das Risiko für Männer ca. $10 \%$ höher ist als das für Frauen.

Das Endometriumkarzinom ist nach dem KRK der zweithäufigste Tumor bei HNPCC. Das Lebenszeitrisiko für weibliche Anlageträgerinnen, ein Endometriumkarzinom zu entwickeln, beträgt 40 $60 \%$ bei einem medianen Erkrankungsalter zwischen 46 und 48 Jahren. Karzinome des Ovars treten bei ungefähr 10-15\% aller Anlageträgerinnen auf. Magenkarzinome kommen bei 2 bis $13 \%$ der HNPCC-Patienten vor und treten durchschnittlich im Alter von 51 - 56 Jahren auf. Erkrankungen vor dem 40. Lebensjahr sind selten. Vorwiegend handelt es sich dabei um Karzinome vom intestinalen Typ. Für Deutschland wurde ein kumulatives Lebenszeitrisiko von 6,8\% bis zum 70. Lebensjahr ermittelt [236]. Das kumulative Lebenszeitrisiko für Dünndarmkarzinome im Rahmen eines HNPCC beträgt 4-8\% [236, 237]. Bei 35 - 50\% der Fälle ist das HNPCC-assoziierte Dünndarmkarzinom im Duodenum lokalisiert [238]. Erkrankungen vor dem 30. Lebensjahr sind selten. Karzinome der oberen ableitenden Harnwege (Ureter/Nierenbecken) treten häufig als Zweit- oder Drittkarzinome auf. Das mittlere Erkrankungsalter für diese Tumoren wird mit 50 - 63 Jahren angegeben. Das Lebenszeitrisiko wird mit $1-12 \%$ angegeben. In einigen Familien wurde eine Häufung von Urothelkarzinomen beobachtet. In einer aktuellen niederländischen Studie [239] wird ein relatives Risiko für Urothelkarzinome des gesamten Urogenitaltrakts (einschließlich der Blasenkarzinome) von 4,2 für männliche und von 2,2 für weibliche Träger (innen) einer pathogenen Keimbahnmutation in einem der MMR-Gene im Vergleich zur niederländischen Allgemeinbevölkerung angegeben. Bislang noch unveröffentlichte Ergebnisse des Deutschen HNPCC-Konsortiums bestätigen diese Angaben.

Das Lebenszeitrisiko für biliäre Tumoren ist bei HNPCC erhöht, aber doch insgesamt relativ niedrig. Pankreaskarzinome werden im Rahmen eines HNPCC selten aber signifikant häufiger als in der Allgemeinbevölkerung beobachtet (Relatives Risiko 8,6; Lebenszeitrisiko 3,7\%) [240, 241]. 
Für Hirntumoren besteht bei HNPCC ein leicht erhöhtes Risiko; histologisch handelt es sich dabei überwiegend um Astrozytome und Glioblastome. Das mediane Erkrankungsalter wird mit 40 54 Jahren angegeben [242-244]. Beim Muir-Torre-Syndrom handelt es sich um eine seltene phänotypische Variante des HNPCC, bei der neben den bereits genannten HNPCC-Tumoren vor allem Talgdrüsenadenome oder -karzinome auftreten [245].

\subsection{Konsensbasierte Empfehlung (2013)}

Eine weiterführende (molekular-)pathologische Untersuchung hinsichtlich HNPCC soll bei mindestens einem erfüllten revidierten Bethesda-Kriterium durchgeführt werden. Hierfür kann entweder an dem Tumorgewebe primär eine qualitätsgesicherte Untersuchung der immunhistochemischen Expression der DNA-Mismatch-Reparatur-Proteine MLH1, MSH2, MSH6 und PMS2 oder eine Untersuchung auf Mikrosatelliten-Instabilität erfolgen. Um ein HNPCC sicher auszuschließen, soll bei unauffälliger immunhistochemischer Expression der DNA-Mismatch-Reparatur-Proteine zusätzlich eine Untersuchung auf Mikrosatellitenstabilität angeschlossen werden. Auf diese kann nur verzichtet werden, wenn immunhistochemisch ein Verlust eindeutig nachgewiesen wurde.

GCP, starker Konsens

\section{Hintergrund}

Ein Algorithmus zur Testung auf einen Mismatch-Reparatur-Defekt im Tumorgewebe ist in $\mathbf{A b b} \mathbf{3}$ dargestellt. Eine Mikrosatelliteninstabilität lässt sich bei etwa 80 \% der Tumorgewebe von Patienten, die die Amsterdam-I/II-Kriterien erfüllen, nachweisen. Dieses Phänomen geht auf den zugrundeliegenden Defekt eines DNA-Reparaturenzyms zurück, durch den bei der Zellteilung entstehende Basenfehlpaarungen nicht mehr korrigiert werden können. Solche Fehlpaarungen treten besonders leicht an Stellen repetitiver kurzer DNA-Fragmente auf (sog. Mikrosatelliten). In Reparatur-defizienten HNPCC-Tumoren findet sich deshalb typischerweise an vielen Stellen des Genoms ein vom Wildtyp der normalen Zellen abweichendes Mikrosatellitenmuster, was zur Bezeichnung „Mikrosatelliteninstabilität“ geführt hat. Bei Patienten aus Familien, in denen die Amsterdam-Kriterien erfüllt sind und deren Tumorgewebe eine Mikrosatellitenstabilität (MSS) zeigt, sollte nach Möglichkeit ein unabhängiger zweiter Tumor aus der Familie untersucht werden.

Bei Patienten, deren Familien die Bethesda-Kriterien erfüllen, ergibt sich bei etwa $30 \%$ der Patienten eine Mikrosatelliteninstabilität und somit ein konkreter Hinweis auf HNPCC. Die klassischen Bethesda-Kriterien wurden 2004 revidiert (Anlage 5.3) [246]. Die Sensitivität der Mikrosatellitenanalyse in HNPCC-assoziierten Tumoren beträgt 79-93\%, die der immunhistochemischen Untersuchung (inkl. MSH6 und PMS2) liegt vergleichbar bei 94\% [247]. Die Immunhistochemie (IHC) ist im Vergleich zur Mikrosatellitenanalyse (MSA) kostengünstiger, schneller durchzuführen und liefert durch den Ausfall eines DNA-Reparaturproteins zusätzlich den Hinweis, in welchem der vier bekannten Mismatch-Reparatur(MMR)-Gene sich die krankheitsverursachende Keimbahnmutation befindet. Sofern die IHC ein eindeutiges Ergebnis ergibt, kann auf eine MSA verzichtet werden. Andernfalls sollte eine MSA angeschlossen werden.

\subsection{Konsensbasierte Empfehlung (2013)}

Bei Patienten mit hoch mikrosatelliteninstabilen Tumoren und einem Ausfall des MLH1-Proteins in der immunhistochemischen Untersuchung soll eine Analyse hinsichtlich einer somatischen
BRAF-Mutation p.Val600Glu durchgeführt werden, um ein HNPCC auszuschließen.

GCP, Konsens

\section{Hintergrund}

Ungefähr 15\% der sporadischen KRK zeigen im Tumorgewebe HNPCC-typische Veränderungen im Sinne einer MSI-H und eines Ausfalls des MLH1-Proteins in der immunhistochemischen Untersuchung (IHC). Ursächlich hierfür ist in der Regel eine somatische Methylierung des MLH1-Promotors. Die Methylierung geht wiederum mit der somatischen Mutation p.Val600Glu im BRAFGen einher [248 - 251]. Bei Tumoren mit MSI-H und einem Ausfall des MLH1-Proteins in der IHC sollte daher zusätzlich eine BRAF-Analyse angeschlossen werden. Durch diese Untersuchung ist es möglich HNPCC-assoziierte von sporadischen KRK zu unterscheiden, da HNPCC-assoziierte KRK keine BRAF-Mutation aufweisen. Für Verwandte ersten Grades eines Patienten mit einem sporadischen MSI-H KRK ist das Risiko ein KRK zu entwickeln leicht erhöht (standardisierte Inzidenz Ratio 1,60) [252]. Es sollten daher die Vorsorgeempfehlungen analog zu den Fällen mit positiver Familienanamnese durchgeführt werden. Möglicherweise ist für Patienten mit einem BRAF-positiven KRK das Risiko für andere Tumoren erhöht (Magen, Ovar) [252].

\subsubsection{Adenomatöse Polyposis-Syndrome}

5.2.1.2.1. Patienten mit klassischer familiär adenomatöser Polyposis (FAP)

\subsection{Statement (2008)}

Patienten mit unbehandelter FAP entwickeln nahezu ausnahmslos ein kolorektales Karzinom.

Level of Evidence 2a, [253]

\section{Hintergrund}

Eine klassische (typische) FAP ist durch das Vorhandensein von mehr als 100 kolorektalen Adenomen charakterisiert. Die Polypenbildung beginnt meist in der zweiten Lebensdekade. Aufgrund der Vielzahl der Adenome beträgt das Karzinomrisiko nahezu $100 \%$.

Die Mehrzahl der Patienten entwickelt darüber hinaus weitere extrakolische intestinale Manifestationen. Klinisch am bedeutsamsten sind Duodenal- bzw. Papillenadenome, die in etwa $75 \%$ aller Patienten auftreten und als Präkanzerose anzusehen sind (s. u.). Weitaus weniger häufig sind Magenadenome mit einer Inzidenz von $<10 \%$ der Patienten mit FAP. Drüsenkörperzysten des Magens, die bei mindestens einem Drittel der FAP-Patienten auftreten, wird gegenwärtig keine potenzielle präneoplastische Potenz zugeschrieben.

Weitere extraintestinale Manifestationen sind abdominale und extraabdominale Desmoidtumoren, Schilddrüsenkarzinome, maligne ZNS-Tumoren (meist Medulloblastome), Hepatoblastome, sowie harmlose, jedoch oft diagnostisch wegweisende Osteome, Epidermoidzysten oder Pigmentanomalien der Retina [253].

\subsection{Patienten mit attenuierter familiärer adenomatöser Polyposis (AFAP)}

\subsection{Statement (2013)}

Die attenuierte FAP (AFAP) ist von dem Krankheitsbild der typischen familiären adenomatösen Polyposis abzugrenzen. Auch hier besteht ein sehr hohes Risiko für ein kolorektales Karzinom, wobei sich Polypen und Karzinome bei den Anlageträgern meist später und häufig im proximalen Kolon entwickeln. 
Level of Evidence 2a, Evidenz aus Aktualisierungsrecherche: [253-261]

\section{Hintergrund}

Die AFAP ist typischerweise durch weniger als 100 kolorektale Adenome und/oder ein etwa 10-15 Jahre späteres Auftreten von Adenomen und KRK als bei klassischer FAP charakterisiert; das Lebenszeitrisiko für die Entwicklung eines KRK ist aber ebenfalls sehr hoch. Extrakolische Manifestationen (z.B. Desmoide) können auftreten [253-258]. Die klinisch definierte AFAP ist aus genetischer Sicht eine heterogene Gruppe. Keimbahnmutationen im APC-Gen (5'- und 3'-Ende des Gens) lassen sich in $15-30 \%$ der Familien nachweisen. Die wichtigste Differenzialdiagnose ist die MUTYH-assoziierte Polyposis (MAP) (s. u.) [259]. Die klinische Abgrenzung gegenüber HNPCC kann im Einzelfall schwierig sein [260]. Daher ist die molekulargenetische Diagnostik (Mikrosatellitenanalyse, APC, MUTYH) unter Umständen bei der klinischen Differenzialdiagnose einer attenuierten FAP sehr hilfreich [261]. Bei einem Großteil der Patienten mit der klinischen Diagnose einer AFAP gelingt derzeit kein Mutationsnachweis, sodass von Mutationen in weiteren, bisher nicht identifizierten Genen ausgegangen werden muss [261].

\subsection{Patienten mit MUTYH-assoziierter Polyposis (MAP)}

\subsection{Statement (2013)}

Die MUTYH-assoziierte Polyposis (MAP) ist die wichtigste Differenzialdiagnose der FAP. Der Phänotyp entspricht meist dem einer AFAP; auch bei der MAP ist das Lebenszeitrisiko für ein KRK sehr hoch. Aufgrund des autosomal-rezessiven Erbgangs besteht aber ein nur geringes Erkrankungsrisiko bei Kindern eines Patienten und heterozygoten Anlageträgern. Die Diagnosestellung ist in der Regel nur durch die molekulargenetische Untersuchung möglich. Level of Evidence 2a, Evidenz aus Aktualisierungsrecherche: [262 - 269]

\section{Hintergrund}

Die durch biallele Keimbahnmutationen im MUTYH-Gen verursachte, autosomal-rezessiv erbliche MAP ist die wichtigste Differenzialdiagnose der APC-assoziierten FAP [262]; sie wird bei 15 $20 \%$ der APC-mutationsnegativen kolorektalen Adenomatosen diagnostiziert [263, 264].

Der kolorektale Phänotyp der MAP ähnelt dem der AFAP: meist treten zwischen 20 und einigen hundert Adenomen auf, das mittlere Diagnosealter ist 45 Jahre (Streuung 12 - 68 Jahre) [265]. Unbehandelt beträgt das KRK-Lebenszeitrisiko etwa 70 - 80\% [266]. Das phänotypische Spektrum der MAP ist noch nicht abschließend geklärt: einige große populationsbasierte Studien an KRK-Patienten zeigten, dass bis zu ein Drittel der biallelen MUTYH-Mutationsträger ein KRK ohne kolorektale Polypen entwickelt [267]. Daneben wurden bei bis zu 50\% der MAP-Patienten hyperplastische Polypen beschrieben [268].

Bei etwa $20 \%$ der Patienten besteht eine Duodenalpolyposis, das Lebenszeitrisiko für ein Duodenalkarzinom beträgt etwa $4 \%$. Extraintestinale Malignome treten insgesamt signifikant häufiger als in der Allgemeinbevölkerung auf (Odds Ratio 1,9) und zeigen eine gewisse Überlappung zum HNPCC; es besteht allerdings kein dominierender Tumor. Typische FAP-assoziierte extraintestinale Tumoren wie Osteome, Desmoide und CHRPE treten nicht auf [269].

\subsubsection{Vorsorgeuntersuchungen}

\subsubsection{HNPCC/Lynch-Syndrom}

5.21. Empfehlung (2013)

Risikopersonen für HNPCC ist mit Erreichen der Einwilligungsfähigkeit (in der Regel ab dem 18. Lebensjahr), jedoch vor dem 25. Lebensjahr eine genetische Beratung zu empfehlen. Sobald die krankheitsverursachende Mutation in der betreffenden Familie bekannt ist, sollten Risikopersonen auf die Möglichkeit einer prädiktiven Testung hingewiesen werden.

Empfehlungsgrad B, Level of Evidence 1c, Evidenz aus Aktualisierungsrecherche: [270, 271], starker Konsens

\subsection{Statement (2008)}

Wenn die krankheitsverursachende Mutation bei einer Risikoperson ausgeschlossen wurde, gelten die allgemeinen Krebsvorsorgemaßnahmen.

Level of Evidence 1c, starker Konsens

\section{Hintergrund}

Anlageträger für ein HNPCC haben Mutationen in den MismatchRepair-Genen. Bislang konnten Keimbahnmutationen in 5 verschiedenen Genen nachgewiesen werden: MSH2, MLH1, MSH6, PMS2 und EPCAM. Fast 86\% der bislang identifizierten Mutationen liegen in den Genen MSH2 und MLH1 [270], etwa 10\% im MSH6Gen und 2\% im PMS2-Gen. Mutationen im EPCAM-Gen werden bei ca. $2 \%$ der Familien gefunden [271]. Vor einer prädiktiven genetischen Untersuchung muss entsprechend des GenDG eine humangenetische Beratung erfolgen. Eine prädiktive Testung ist in der Regel nur möglich, wenn in der Familie bei einem bereits erkrankten Mitglied eine zweifelsfrei pathogene Mutation nachgewiesen wurde. Der Nachweis von Polymorphismen oder Mutationen mit unklarer pathogenetischer Bedeutung eignet sich nicht als Grundlage für eine prädiktive genetische Diagnostik.

\subsection{Konsensbasierte Empfehlung (2013)}

HNPCC-Patienten und Risikopersonen sollen in der Regel ab dem 25. Lebensjahr jährlich koloskopiert werden.

GCP, Konsens

\section{Hintergrund}

HNPCC-Patienten haben ein deutlich erhöhtes Karzinomrisiko. Einen Überblick über die empfohlenen Krebsfrüherkennungsuntersuchungen gibt $\bullet$ Tab. 6. Kolonkarzinome bei HNPCC-Patienten treten im Median im 44. Lebensjahr auf. Die Erkrankungswahrscheinlichkeit steigt ab dem 30. Lebensjahr deutlich an. Bei sehr früher Manifestation kolorektaler Karzinome in der Familie sollte abweichend von der o.g. Empfehlung die erste Koloskopie 5 Jahre vor dem jüngsten Manifestationsalter in der Familie erfolgen. Mehr als 50\% der HNPCC-assoziierten Karzinome befinden sich im rechtsseitigen Kolon [243]. Aus diesem Grunde reicht eine Rektoskopie bzw. Rektosigmoidoskopie als Vorsorgeuntersuchung nicht aus. Eine prospektive Studie konnte eine signifikante Reduktion der Mortalität und auch Inzidenz von KRK um mehr als jeweils $60 \%$ bei 3-jährlichen Untersuchungsintervallen nachweisen [272]. Aufgrund einer beschleunigten Tumorprogression mit Intervallkarzinomen in etwa $4 \%$ aller Patienten bei 2- bis 3-jährlichen Untersuchungsabständen wird ein jährliches Intervall empfohlen [272 - 274] (৫ Tab.6). In der prospektiven Studie des Deutschen HNPCC-Konsortiums mit 1jährlichem Intervall weisen die unter Überwachung asymptomatisch detektierten CRC eine signifikant günstigere Stadienverteilung auf [229]. 
Die Stadienverteilung und damit auch Prognose HNPCC-assoziierter kolorektaler Karzinome, die im Rahmen eines Vorsorgeprogrammes entdeckt werden, ist signifikant günstiger verglichen zu Karzinomen, die aufgrund von Symptomen der Erkrankung diagnostiziert worden sind [275]. Die Koloskopie kann als Chromoendoskopie erfolgen. In prospektiven Studien wurde durch eine Chromoendoskopie eine signifikante Steigerung der Adenomdetektionsrate gesehen [276-278]. Es ist aber gegenwärtig unklar, ob dadurch die Intervallkarzinomrate oder Mortalität günstig beeinflusst wird.

\subsection{Empfehlung (2013)}

Bei weiblichen HNPCC-Patienten und Risikopersonen sollte ab dem 25. Lebensjahr zusätzlich zur jährlichen gynäkologischen Untersuchung ein transvaginaler Ultraschall im Hinblick auf Endometrium- und Ovarialkarzinome durchgeführt werden.

Empfehlungsgrad B, Level of Evidence 4, [242, 279 - 282], starker Konsens

\subsection{Konsensbasierte Empfehlung (2013)}

Bei weiblichen HNPCC-Patienten und Risikopersonen sollte ab dem 35. Lebensjahr zusätzlich jährlich eine Endometriumbiopsie durchgeführt werden.

GCP, Konsens

\section{Hintergrund}

Diese Empfehlungen ergeben sich aus dem natürlichen Verlauf des HNPCC (s. o.). Bei Anlageträgerinnen beträgt das Risiko bis zum 70. Lebensjahr ein Endometriumkarzinom zu entwickeln 40 bis $60 \%$, für ein Ovarialkarzinom ungefähr $10-15 \%$ [242, 279]. Die bisher publizierten Studien hinsichtlich der Effektivität der Früherkennung des Endometriumkarzinoms bei HNPCC-Patientinnen zeigten deutliche Hinweise, dass der transvaginale Ultraschall (TVU) als Screening-Untersuchung zur Früherkennung des Endometriumkarzinoms insbesondere prä- und perimenopausal ungeeignet ist [280 - 282]. Da die Endometriumbiopsie mit der Pipelle-Methode in Ergänzung zum TVU aus den Literaturdaten als die sinnvollste Alternative hervorgeht und diese auch bereits in internationalen Empfehlungen für HNPCC-Patientinnen propagiert wurde [283], ist die Empfehlung zur jährlichen Endometriumbiopsie ab dem 35. Lebensjahr sinnvoll ( $\bullet$ Tab. 7). Bei Patienten mit abgeschlossener Familienplanung sollte die Möglichkeit einer prophylaktischen Hysterektomie und ggf. Adnektomie diskutiert werden (s. Empfehlung 5.12.).

\subsection{Konsensbasierte Empfehlung (2013)}

Bei HNPCC-Patienten und Risikopersonen mit HNPCC sollte ab dem 35. Lebensjahr zusätzlich regelmäßig eine ÖGD durchgeführt werden.

GCP, Konsens

\section{Hintergrund}

HNPCC-assoziierte Magenkarzinome werden im Median im 54. Lebensjahr diagnostiziert, sie treten in nur $2 \%$ vor dem 35. Lebensjahr auf. Für Deutschland wurde ein kumulatives Lebenszeitrisiko von $6,8 \%$ bis zum 70 . Lebensjahr ermittelt [236]. Eine familiäre Häufung (mindestens 2 Betroffene mit Magenkarzinomen) ließ sich in nur 26\% der MLH1- und MSH2-Mutationsträger nachweisen [284]. Daher erscheint eine ÖGD für alle Anlageträger und Risikopersonen ab dem 35. Lebensjahr sinnvoll (๑ Tab.6). Der Beginn der ÖGD mit 35 Jahren wird auch vorge- schlagen, da das Dünndarmkarzinomrisiko das Magenkrebsrisiko übersteigt und Erkrankungen ab dem 30. Lebensjahr auftreten. Das kumulative Lebenszeitrisiko für Dünndarmkarzinome im Rahmen eines HNPCC beträgt 4-8\% [236, 237]. Dabei sind $35-50 \%$ der Dünndarmkarzinome im Duodenum lokalisiert [238]. Die Empfehlung der S3-Leitlinie zum Magenkarzinom wird aufgrund der Datenlage künftig zu präzisieren sein. Zur Frage des Untersuchungsintervalls gibt es keine Daten; in Analogie zur beschleunigten Tumorprogression kolorektaler Karzinome auf dem Boden eines hereditären MMR-Defekts wird ein jährliches Intervall als sinnvoll erachtet.

Wegen des erhöhten Risikos für Urothelkarzinome und hepatobiliäre Karzinome wurde früher eine jährliche Oberbauchsonografie empfohlen. Der Nutzen ist aber nicht belegt und vom kurativen Potential fraglich. Sie wird daher nicht länger allgemein empfohlen. Der Nutzen einer urinzytologischen Untersuchung ist nicht belegt und wird daher seit 2004 nicht mehr allgemein empfohlen.

\subsection{Empfehlung (2013)}

Eine medikamentöse Prävention bei HNPCC sollte nicht durchgeführt werden.

Empfehlungsgrad A, Level of Evidence2b, Evidenz aus Aktualisierungsrecherche: [285, 286], Konsens

\section{Hintergrund}

Zur Chemoprävention bei HNPCC liegen bisher nur Daten einer prospektiv randomisierten Studie vor. Die CAPP2-Studie hat in einem $2 \times 2$-Design den Einsatz von $600 \mathrm{mg}$ Acetylsalicylsäure und von nicht resorbierbarer Stärke geprüft. Die primäre Analyse der definierten Endpunkte ergab keinen Nachweis eines signifikanten Effekts von ASS [285]. Nach einem längeren Follow-up von 55,7 Monaten fand sich für die Subgruppe von HNPCC-Patienten, die $600 \mathrm{mg}$ ASS für mindestens 2 Jahre eingenommen hatten eine signifikante Senkung der Inzidenz kolorektaler Karzinome (Hazard Ratio 0,41 [95\%CI 0,19-0,86], p=0,02) sowie eine nicht signifikante Senkung von anderen HNPCC-assoziierten Karzinomen (Hazard Ratio 0,47 [95\%CI 0,21-1,06], p=0,07) [286]. Die untersuchte ASS Dosis erscheint mit $600 \mathrm{mg}$ hoch mit entsprechend zu befürchtender erhöhter Nebenwirkungsrate. Die Effektivität niedrigerer ASS-Dosen ist derzeit für HNPCC-Patienten nicht geklärt und soll in einer Nachfolgestudie (CAPP-3) untersucht werden. Es sollte angestrebt werden, möglichst viele HNPCC-Patienten in diese Studie einzuschließen. Bis die Ergebnisse dieser Studie vorliegen, sollte keine allgemeine medikamentöse Prävention mit ASS bei HNPCC-Patienten erfolgen.

\subsection{Konsensbasierte Empfehlung (2013)}

Eine prophylaktische Kolektomie bzw. Proktokolektomie bei HNPCC-Mutationsträgern soll nicht durchgeführt werden. Eine subtotale Kolektomie bei Karzinom sollte nicht generell durchgeführt, aber individuell mit dem Patienten besprochen werden. GCP, starker Konsens

\subsection{Empfehlung (2013)}

Die koloskopische Überwachung des Patienten nach onkologischer Resektion soll zusätzlich zur üblichen Nachsorge auch postoperativ nach dem gleichen Muster wie vor der Operation fortgesetzt werden.

Empfehlungsgrad A, Level of Evidence 2a, Evidenz aus Aktualisierungsrecherche: [272, 273, 287 - 290], starker Konsens 


\section{Hintergrund}

Da durch regelmäßige Vorsorgeuntersuchungen Karzinome bei fast allen Patienten im Stadium UICC I/II oder sogar als prämaligne Adenome entdeckt werden $[272,273]$ und die Penetranz unvollständig ist, wird von einer prophylaktische Kolektomie bzw. Proktokolektomie abgeraten. Individuelle Konstellationen wie endoskopisch nicht abtragbare Adenome, regelhaft schwierige Koloskopien, nicht ausreichende Kolonvorbereitung trotz adäquater Abführmaßnahmen und fehlende Compliance können eine Empfehlung zu einer prophylaktischen Kolektomie rechtfertigen.

Bei Nachweis eines Karzinoms werden die Patienten nach tumorchirurgischen Gesichtspunkten im Sinne einer onkologischen Resektion operiert (s. auch Kapitel 7). Das Risiko eines kolorektalen Karzinoms im verbliebenen Dickdarm und das Risiko von extrakolischen Neoplasien bleiben jedoch erhöht, sodass diese Patienten einer intensiven postoperativen Nachsorge zugeführt werden müssen. In dieser sollte die Tumornachsorge für sporadische KRK mit dem HNPCC-spezifischen Früherkennungsprogramm für KRK und extrakolische Tumoren kombiniert werden. Ob eine erweiterte prophylaktische Resektion zur Prophylaxe metachroner KRK einer engmaschigen Überwachung überlegen ist, ist derzeit unklar. Die bisherigen Daten aus retrospektiven Fallserien sind unzureichend, und aufgrund nationaler Unterschiede im Bezug auf das Screening-Intervall nicht auf Deutschland übertragbar [287-290].

\subsection{Konsensbasierte Empfehlung (2013)}

Mit Patientinnen mit Lynch- und HNPCC-Syndrom sollte mit 40 Jahren, bzw. 5 Jahre vor dem frühesten Erkrankungsalter in der Familie, eine prophylaktische Hysterektomie und ggf. eine Ovarektomie besprochen werden.

GCP, Konsens

\section{Hintergrund}

Dieses Vorgehen basiert auf der Expertenmeinung der Arbeitsgemeinschaft Gynäkologische Onkologie (AGO) der Deutschen Krebsgesellschaft. Der Eingriff sollte möglichst erst nach Abschluss der Familienplanung erfolgen. Eine retrospektive Studie konnte einen deutlichen Rückgang in der Inzidenz der Endometrium- und Ovarialkarzinome bei diesen Patientinnen zeigen [291]. Nach einer prophylaktischen Ovarektomie sollte eine Hormontherapie (HRT) durchgeführt werden. Nach einer Hysterektomie kann die HRT auch nur mit Östrogenen erfolgen, wodurch die Nebenwirkungen der Therapie gesenkt werden.

\subsubsection{Adenomatöse Polyposis-Syndrome}

5.2.2.2.1. Patienten mit klassischer familiärer adenomatöser Polyposis (FAP)

\subsection{Empfehlung (2013)}

Verwandte eines FAP-Patienten, die aufgrund des autosomal-dominanten Erbgangs als Mutationsträger in Betracht kommen, werden als Risikopersonen bezeichnet. Bei diesen sollte ab dem 10. Lebensjahr im Anschluss an eine humangenetische Beratung der Familie eine prädiktive genetische Diagnostik empfohlen werden, soweit die zugrunde liegende APC-Keimbahnmutation in der Familie identifiziert werden konnte.

Empfehlungsgrad B, Level of Evidence 4, [120, 292], starker Konsens

\subsection{Empfehlung (2008)}

Wurde die in der Familie identifizierte Mutation bei Risikopersonen (Kinder, Geschwister oder Eltern eines FAP-Patienten) ausgeschlossen, ist eine gesonderte Vorsorge nicht mehr notwendig. Empfehlungsgrad A, Level of Evidence 1c, Keine Angabe, starker Konsens

\subsection{Empfehlung (2013)}

Risikopersonen, bei denen die Mutation bestätigt oder nicht ausgeschlossen werden konnte, sollen ab dem 10. Lebensjahr jährlich rekto-sigmoidoskopiert werden. Bei Nachweis von Adenomen soll eine komplette Koloskopie erfolgen und bis zur Proktokolektomie jährlich wiederholt werden (s.u.).

Empfehlungsgrad A, Level of Evidence 4, [191, 292, 293] [120], starker Konsens

\section{Hintergrund}

Die humangenetische Beratung erfolgt bei nicht einwilligungsfähigen Personen gemeinsam mit den Erziehungsberechtigten. Die Einleitung der humangenetischen Diagnostik vor dem 10. Lebensjahr ist selten notwendig, da kolorektale Karzinome bei FAP-Anlageträgern vor dem 15. Lebensjahr sehr selten sind [292]. Die molekulargenetische Untersuchung erfolgt in der Regel mittels direktem Mutationsnachweis im APC-Gen, in seltenen Fällen mit aussagekräftiger familiärer Konstellation kann die Anlageträgerschaft auch indirekt durch Kopplungsanalyse nachgewiesen werden. Eine sichere prädiktive Testung kann nur bei zuvor identifizierter pathogener Keimbahnmutation bei einem betroffenen Familienmitglied erfolgen und muss in eine humangenetische Beratung eingebettet sein [120] (GenDG). Ein Mutationsnachweis gelingt bei ca. 70-80\% der Patienten. Als weitere Methode zur Identifizierung von Genträgern kann eine Augenhintergrundspiegelung durchgeführt werden. Die Augenhintergrundspiegelung zur Identifizierung einer kongenitalen Hypertrophie des retinalen Pigmentepithels (CHRPE) hat durch die prädiktive DNA-Testung jedoch an Bedeutung verloren.

Bei der klassischen FAP werden immer auch Polypen im Rektum und Sigma beobachtet. Sind Rektumpolypen nachgewiesen worden, so können weiter proximal weitere Adenome oder sogar Karzinome vorhanden sein. In diesem Falle soll kurzfristig eine komplette Koloskopie durchgeführt werden, die je nach Befund in mindestens jährlichen Abständen wiederholt werden sollte. In Familien, in denen eine genetische Testung nicht durchgeführt wurde oder nicht aussagekräftig war, ist allen Risikopersonen die endoskopische Vorsorge ab dem 10. Lebensjahr zu empfehlen [191, 292, 293]. Bei bestimmten Mutationen, früher Karzinommanifestation in der Familie oder assoziierten Symptomen ist der Beginn der Vorsorge bereits zu einem früheren Zeitpunkt in Erwägung zu ziehen.

\subsection{Empfehlung (2013)}

Patienten mit klassischer FAP sollten prophylaktisch und unabhängig vom Ergebnis der molekulargenetischen Testung - wann immer möglich kontinenzerhaltend - proktokolektomiert werden, wenn vertretbar erst nach Abschluss der Pubertät.

Empfehlungsgrad B, Level of Evidence 1c, Evidenz aus Aktualisierungsrecherche: [261, 294 - 299], starker Konsens

\subsection{Konsensbasierte Empfehlung (2013)}

Nach einer Proktokolektomie soll regelmäßig eine Pouchoskopie erfolgen. Bei Patienten mit erhaltenem Rektumstumpf soll regelmäßig eine Rektoskopie durchgeführt werden. Das Intervall hängt 
vom Untersuchungsergebnis (Anzahl, Größe und Histologie nachgewiesener Neoplasien) ab und sollte 12 Monate nicht überschreiten. GCP, starker Konsens

\section{Hintergrund}

Die rechtzeitige Proktokolektomie ist zur Verhinderung des kolorektalen Karzinoms entscheidend [294-298]. Die Relevanz des molekulargenetischen Befundes für therapeutische Entscheidungen ist begrenzt, da der Nachweis einer Mutation nur selten eine individuelle Abschätzung des Krankheitsverlaufs erlaubt und bei einem nennenswerten Teil der klinisch sicher erkrankten Personen keine ursächliche Mutation identifiziert werden kann. Der Polyposis-Patient muss unabhängig vom Ergebnis der Mutationssuche adäquat behandelt werden, notwendige chirurgische Maßnahmen sollten deshalb auch bei fehlendem Mutationsnachweis und ggf. noch vor Abschluss der Mutationssuche durchgeführt werden [261]. Als Orientierung ist ein Operationszeitpunkt zwischen Abschluss der Pubertät und dem 20. Lebensjahr für eine prophylaktische Proktokolektomie anzustreben. Der OPZeitpunkt muss sich jedoch individuell nach Alter, Anamnese und endoskopischem/histologischem Befund (Zahl der Polypen und Dysplasiegrad) richten [297-299]. Nach der Beobachtung zum natürlichen Verlauf der FAP entsteht das Karzinom im Median im 36. Lebensjahr [300]. Mit dem Patienten sollte das mögliche Belassen eines Rektumstumpfs diskutiert werden (ileorektale Anastomose, IRA). Hierbei ist zu beachten, dass nach Kolektomie mit Belassung des Rektums das Risiko der Entstehung eines Rektumstumpfkarzinoms ca. 13\% nach 25 Jahren beträgt [301], während die Langzeitprognose nach IPAA (ileo-pouchanaler Anastomose) bessere Langzeitergebnisse hinsichtlich der Karzinomprophylaxe aufweist [302 - 305]. Aus diesem Grunde wird bei der klassischen FAP die Proktokolektomie mit IPAA empfohlen. Die Operation sollte in entsprechend erfahrenen Zentren durchgeführt werden. Die Durchführung einer Proktokolektomie mit endständigem Ileostoma kann heute größtenteils vermieden werden.

Da viele Patienten Polypen im Bereich des Pouches nahe der ileoanalen Anastomose entwickeln, die maligne entarten können, wird postoperativ eine jährliche Pouchoskopie empfohlen. Sofern keine Proktokolektomie erfolgte, ist die engmaschige Kontrolle des Rektumstumpfs in maximal 12-monatlichen Intervallen erforderlich, ggf. mit Abtragung neu auftretender Polypen.

\subsection{Konsensbasierte Empfehlung (2013)}

Eine ÖGD und Duodenoskopie (Seitblickoptik) mit besonderer Inspektion der Papillenregion sollte spätestens ab dem 25.-30. Lebensjahr durchgeführt werden. Bei unauffälligem Befund wird ein 3-Jahres-Intervall empfohlen. Das Intervall sollte in Abhängigkeit vom Schweregrad vorhandener Adenome (Spigelman-Klassifikation) auf bis zu einem Jahr verkürzt werden.

Bei Nachweis von Duodenal-/Papillenadenomen ist die Indikation zur endoskopischen Polypektomie zu überprüfen.

Bei schwergradiger Duodenalpolyposis (Spigelman IV) und invasivem nicht fernmetastasiertem Karzinom besteht eine Indikation zur operativen Resektion.

GCP, Konsens

\section{Hintergrund}

Das Lebenszeitrisiko Duodenalpolypen zu entwickeln beträgt für FAP-Patienten zwischen 80 und 90\% [231, 232]. Weniger als $10 \%$ der Patienten weisen Magenadenome auf, über 50\% Drüsenkör- perzysten des Magens. Magenkarzinome scheinen hingegen nicht signifikant häufiger als in der Normalbevölkerung zu sein [306]. Hinsichtlich der Ausprägung der Duodenal-Polyposis sollte die Spigelman-Klassifikation ( $\bullet$ Tab.7) herangezogen werden [307]. Das durchschnittliche Alter der Patienten mit schwerer Duodenalpolyposis liegt bei 43 (Range 24 - 65) Jahren [233]. Insgesamt scheint das Wachstumsverhalten der Duodenaladenome langsamer als das von kolorektalen Adenomen zu sein [308, 309] und hängt mehr vom zunehmenden Alter (>40 Jahre zunehmend) als vom initialen Stadium ab [310]. Der Mutationsort (Codon 279 - 1309) korreliert mit dem Schweregrad der Polyposis im Duodenum aber wohl nicht mit der Wahrscheinlichkeit, dass sich hochgradige Dysplasien entwickeln [311, 312]. Das Lebenszeitrisiko für ein Duodenalkarzinom beträgt für Patienten mit FAP zwischen 3 und 4\% [313, 314] und ist somit bis zu 300fach höher als in der Normalbevölkerung [315]. Das Risiko, dass bereits ein invasives Karzinom vorliegt, richtet sich nach der Ausprägung der Duodenalpolyposis. So liegt das Risiko für ein invasives Karzinom bei Spigelman II und III bei jeweils $2 \%$, demgegenüber bei Spigelman IV bei 36\% [316].

Sinn einer endoskopischen Überwachung kann nicht die Entfernung sämtlicher Polypen sein, sondern muss auf die Entdeckung von relevanten Neoplasien (high-grade intraepitheliale Neoplasie, villöse oder tubulovillöse Adenome) ausgerichtet sein. Eine endoskopische Sanierung bis hin zur Polypenfreiheit ist in der Regel nicht sinnvoll. Alle Polypen $>1 \mathrm{~cm}$ sollten, sofern technisch möglich, abgetragen werden. Bei kleineren Polypen sollten die größten Polypen abgetragen werden, um durch die histologische Untersuchung einen Spigelman-Score festlegen zu können.

Für FAP-Patienten mit leichtgradiger Duodenalpolyposis (Spigelman I und II) scheint ein Untersuchungsintervall von 3 Jahren ausreichend [308]. Aufgrund des hohen Karzinomrisikos im Spigelman-Stadium IV muss hier ein chirurgisches Vorgehen empfohlen werden, wobei der pankreaserhaltenden Duodenektomie, auch aufgrund der geringeren Morbiditätsrate gegenüber der Pankreatikoduodenektomie der Vorzug zu geben ist [317, 318]. Die operative Duodenotomie und Polypektomie kann wegen der hohen Rezidivrate nicht empfohlen werden [319, 320]. Prinzipiell lässt sich auch nach extensivem chirurgischem Vorgehen das Wiederauftreten von Adenomen nicht verhindern [319]. Auch ist derzeit nicht klar, ob die regelmäßige Vorsorgeuntersuchung des Duodenums lebensverlängernd ist [313]. Zum Vorgehen bei FAP-assoziierten Papillenadenomen gibt es unterschiedliche Vorgehensweisen. Insgesamt existieren zu dieser Problematik bei FAP nur sehr wenige Publikationen. Während z.T. bei jedem Patienten bei jedem Papillenadenom eine Papillektomie favorisiert wird [321, 322], favorisieren andere Gruppen bei kleinen Adenomen eine Überwachungsstrategie und eine Papillektomie nur bei Progress (Größe, Histologie) oder eingetretenen bzw. drohenden Komplikationen (z. B. Cholestase, Pankreatitis) [308, 309]. Zusammenfassend erscheint das folgende Überwachungsprogramm sinnvoll, wobei es jeweils der individuellen Situation angepasst werden sollte: Spigelman I und II: Kontrolle mindestens alle 3 Jahre, gegebenenfalls Polypektomie, Spigelman III: Kontrolle jährlich, gegebenenfalls Polypektomie, Spigelman IV: Operation.

\subsection{Konsensbasierte Empfehlung (2013)}

Bei vorliegender Therapieindikation (Symptomatik, Progredienz) sollten Desmoide bei FAP in erster Linie mit einer Kombinationstherapie aus Sulindac und Tamoxifen behandelt werden. Das Vorgehen 
bei progredienten Desmoiden unter dieser medikamentösen Therapie soll interdisziplinär abgestimmt werden. Als Optionen kommen Chemotherapie, Chirurgie und Radiatio in Betracht.

GCP, Konsens

\section{Hintergrund}

Desmoidtumoren treten bei $10-30 \%$ aller Patienten mit einer FAP auf. Das Risiko für die Entwicklung eines Desmoidtumors ist für Frauen höher als für Männer. Neben einer erkennbaren Geno-/Phänotyp-Korrelation (APC-Mutation Kodon >1300) [323325] wird ein chirurgisches Trauma als Triggerfaktor angesehen. Etwa 50\% der Desmoide treten intraabdominell und hier vor allem mesenterial auf und verursachen durch das lokal infiltrative Wachstum oft erhebliche Probleme. Aus diesem Grunde ist insbesondere bei Patienten mit einer positiven Familienanamnese oder einer distalen APC-Mutation vor einer Proktokolektomie auf das Vorhandensein von Desmoiden zu achten und die Indikation für eine Proktokolektomie möglichst spät zu stellen. Ein regelmäßiges Screening auf Desmoide wird bei beschwerdefreien Patienten nicht empfohlen. Präoperativ kann ein Screening auf Desmoide mit einem geeigneten bildgebenden Verfahren durchgeführt werden. Asymptomatische und nicht größenprogrediente Desmoidtumoren bedürfen oft keiner Therapie. In einem systematischen Review publizierter klinischer Studien zur medikamentösen, nicht zytotoxischen Therapie sind eine Therapie mit Sulindac ( $300 \mathrm{mg}$ ) oder Tamoxifen $(40-120 \mathrm{mg}$ ) oder eine Kombinationstherapie am besten hinsichtlich ihrer Wirksamkeit belegt [326]. Auch zu Raloxifen liegen vergleichbare Daten aus einer kleinen Fallserie vor [327]. Progrediente Tumoren unter Sulindac oder antihormoneller Therapie sollten mit einer Chemotherapie (Doxorubicin und Dacarbazine oder Methotrexat und Vinblastin) oder einer Strahlentherapie behandelt werden [328 - 330]. Ergebnisse zur Resektion sind kontrovers [331]. Vielfach wird insbesondere bei intraabdominellen Desmoiden über inkomplette Resektionen und hohe Rezidivraten berichtet [332 - 335].

Bei Bauchwanddesmoiden scheint in vielen Fällen ein chirurgisches Vorgehen mit Erreichen einer R0-Resektion und Rezidivfreiheit möglich zu sein [333].

\subsection{Konsensbasierte Empfehlung (2013)}

Eine jährliche Sonografie der Schilddrüse kann ab dem 15. Lebensjahr bei weiblichen FAP-Patienten durchgeführt werden. GCP, Konsens

\section{Hintergrund}

Das Lebenszeitrisiko für Patienten mit einer FAP, ein Schilddrüsenkarzinom zu entwickeln, beträgt 1-12\%. Ungefähr $95 \%$ aller berichteten Schilddrüsenkarzinome sind bei Frauen aufgetreten; das Risiko ist daher vor allem für weibliche Anlageträger erhöht. Die Diagnose erfolgt im Mittel zwischen dem 24. und 33. Lebensjahr. Erkrankungen vor dem 15. Lebensjahr sind selten [336]. Karzinome treten oft multifokal und zum Teil bilateral auf. Histologisch liegt in der Regel die kribiforme Variante eines papillären Schilddrüsenkarzinoms vor.

Zwei prospektive [337, 338] und eine retrospektive Studie [339] haben den Stellenwert eines einzeitigen Ultraschall-Screenings untersucht. Benigne Schilddrüsen-Knoten wurden dabei in 20 $79 \%$ entdeckt. Schilddrüsenkarzinome wurden mit einer Prävalenz von 2,6-7,6\% diagnostiziert. In der größten Studie wurde keines der Karzinome durch die Anamnese oder Palpation der Schilddrüse entdeckt, sondern alle über die Sonografie [337]. Daher kann ein Ultraschall-Screening für weibliche FAP-Patienten ab dem 15. Lebensjahr durchgeführt werden. Untersuchungen zum geeigneten Untersuchungsintervall liegen bisher nicht vor. Die Prognose FAP-assoziierter Schilddrüsenkarzinome ist gut; Todesfälle sind aber beschrieben [336, 340, 341]. Inwieweit durch das Screening eine Mortalitätssenkung erreicht werden kann, ist unklar. Möglicherweise kann die Häufigkeit der Notwendigkeit einer adjuvanten Radiotherapie gesenkt werden, wenn vermehrt Mikrokarzinome detektiert werden.

Das Hepatoblastom ist eine sehr seltene Manifestation einer FAP. Weniger als 0,5\% aller Kinder von FAP-Patienten entwickeln fast ausschließlich vor dem 10. Lebensjahr ein Hepatoblastom [342]. Dabei scheint das Risiko für Jungen höher zu sein. In einem Teil der Fälle gibt es eine positive Familienanamnese [343]. Aufgrund der Seltenheit und der unklaren Datenlage, ob durch ein Screening die Prognose von Hepatoblastompatienten verbessert werden kann, wird ein Screening nicht generell empfohlen [344, 345].

\subsection{Empfehlung (2013)}

Eine medikamentöse Behandlung von Adenomen im unteren und oberen Gastrointestinaltrakt sollte nicht generell empfohlen werden. Empfehlungsgrad B, Level of Evidence 2b, Evidenz aus Aktualisierungsrecherche: [346 - 354], starker Konsens

\section{Hintergrund}

Sulindac reduziert die Anzahl kolorektaler Adenome um mehr als $50 \%$ [346 - 350]. Es verhindert jedoch nicht die Polypenneubildung [351]. Weiterhin wurden unter Sulindac-Therapie Karzinome im Rektumstumpf beobachtet. Sulindac ist in Deutschland nicht zugelassen. Eine Chemoprävention mit Sulindac bei FAP kann in Einzelfällen als additive Behandlung nach subtotaler Kolektomie durchgeführt werden, um die rektale Polypenlast zu reduzieren. Eine endoskopische Überwachung ist jedoch zwingend. Der selektive COX-2-Hemmer Celecoxib, der zu einer Reduktion rektaler Adenome führt [352], wurde zur Chemoprävention bei FAP als Ergänzung zu chirurgischen Maßnahmen und weiteren endoskopischen Kontrollen zugelassen. Celecoxib in hohen Dosierungen von 400-800 mg reduziert die kolorektale Polypenzahl um 28\% [352] und beeinflusst auch eine Duodenalpolyposis [353]. Es ist allerdings ungeklärt, ob die Einnahme auch das Risiko der Karzinomentstehung bei diesen Patienten senkt. COX-2Hemmer sind mit einer erhöhten kardiovaskulären Ereignisrate assoziiert [354]. Der Stellenwert der COX-2-Inhibitoren ist aufgrund der kardiovaskulären Nebenwirkungen derzeit unklar und diese sollten gegenwärtig nur in ausgewählten Fällen bei strenger Indikationsstellung (Nutzen-Risiko-Abwägung) verwendet werden. Das für die FAP zugelassene Präparat mit dem Wirkstoff Celecoxib wurde im April 2011 vom Hersteller aufgrund mangelnder Rekrutierung einer von der europäischen Arzneimittelbehörde (EMA) geforderten Post-Zulassungsstudie vom Markt genommen. Die Verwendung von COX-2-Inhibitoren könnte bei selektionierten Patienten zur Verzögerung einer Kolektomie, bei subtotaler Kolektomie zur Reduzierung der Rektumpolypenlast, bei schwerer Duodenalpolyposis erhöhtem Operationsrisiko oder erhöhtem Perforations- und Blutungsrisiko bei Polypektomie in Einzelfällen gerechtfertigt werden.

\subsection{Patienten mit attenuierter familiärer adenomatöser Polyposis}

\subsection{Empfehlung (2008)}

Ein Patient mit einer attenuierten FAP sollte in Abhängigkeit von Alter, Polypenzahl und histologischem Befund therapiert werden. Bei endoskopisch nicht beherrschbarer Polyposis ist eine Kolekto- 
mie indiziert. Patienten, die nicht kolektomiert sind, sollten zeitlebens jedes Jahr koloskopiert werden.

Empfehlungsgrad B, Level of Evidence 4 [254, 255, 257, 258, 355 - 358], starker Konsens

\subsection{Empfehlung (2008)}

Risikopersonen aus Familien mit attenuierter FAP sollten im Rahmen der Vorsorgeuntersuchung im Alter von 15 Jahren erstmals koloskopiert werden. Finden sich keine Polypen, sollten diese Personen ab dem 20. Lebensjahr jährlich koloskopiert werden.

Empfehlungsgrad C, Level of Evidence 4, [356 - 358], starker Konsens

\section{Hintergrund}

Bei Patienten mit einer attenuierten FAP treten Polypen oft deutlich später und seltener auf als bei der klassischen FAP. Die Diagnose eines KRK in der Adoleszenz wurde kasuistisch berichtet [355]. Die Polypen sind häufig im rechtsseitigen Kolon lokalisiert. Aus diesem Grunde muss eine komplette Koloskopie zur Früherkennung durchgeführt werden [254, 255, 257, 258]. Da die klinische Ausprägung stark variieren kann, ist die Therapieentscheidung individuell abzuwägen. Bei Patienten mit bestehender Operationsindikation, aber weniger als 5 Rektumpolypen, ist unter Umständen eine ileorektale Anastomose mit Belassen eines Rektumstumpfs vertretbar. Da extrakolische Manifestationen genau wie bei der klassischen FAP auftreten können [356-358], gelten diesbezüglich die Empfehlungen für die klassische FAP. Bis zu welchem Alter Vorsorgeuntersuchungen bei Risikopersonen mit unauffälligem endoskopischem Befund fortgeführt werden sollen, ist aufgrund der Datenlage derzeit unklar.

\subsection{Patienten mit MUTYH-assoziierter Polyposis (MAP)}

5.42. Konsensbasierte Empfehlung (2013)

Eine Testung im MUTYH-Gen soll bei Patienten durchgefüht werden, bei denen klinisch eine attenuierte adenomatöse Polyposis besteht und keine krankheitsverursachende Mutation im APC-Gen nachgewiesen wurde.

GCP, Konsens

\section{Hintergrund}

Die MUTYH-assoziierter Polyposis (MAP) ist die wichtigste Differenzialdiagnose der FAP. Der Phänotyp entspricht meist dem einer AFAP; auch bei der MAP ist das Lebenszeitrisiko für ein KRK sehr hoch. Aufgrund des autosomal-rezessiven Erbgangs besteht aber ein nur geringes Erkrankungsrisiko bei Kindern eines Patienten und heterozygoten Anlageträgern. Die Diagnosestellung ist in der Regel nur durch die molekulargenetische Untersuchung möglich. Etwa 50 \% der Patienten haben bei Diagnose einer MAP bereits ein manifestes KRK, bei einem Drittel dieser Fälle wurden synchrone oder metachrone KRK beobachtet. Die Polypen treten im gesamten Dickdarm auf, KRKs finden sich in über $50 \% \mathrm{im}$ rechtsseitigen Kolon. Das Auftreten von MAP-assoziierten KRKs vor dem 29. Lebensjahr ist eine Rarität.

\subsection{Konsensbasierte Empfehlung (2013)}

Geschwister eines MAP-Patienten haben aufgrund des autosomalrezessiven Erbgangs ein Erkrankungsrisiko von 25\% und werden als Risikopersonen bezeichnet. Diesen sollte ab dem 18. -20. Lebensjahr im Anschluss an eine humangenetische Beratung eine prädiktive genetische Diagnostik empfohlen werden.

GCP, starker Konsens

\subsection{Konsensbasierte Empfehlung (2013)}

Werden beide MUTYH-Mutationen des Indexpatienten bei seinen Geschwistern ausgeschlossen, ist eine gesonderte Vorsorge bei diesen nicht mehr notwendig.

GCP, starker Konsens

\subsection{Konsensbasierte Empfehlung (2013)}

Für Verwandte eines MAP-Patienten, bei denen lediglich eine der beim Indexpatienten nachgewiesenen MUTYH-Mutationen vorliegt (heterozygote Anlageträger), werden Vorsorgeuntersuchungen wie bei erstgradig Verwandten eines Patienten mit sporadischem KRK empfohlen (s. 5.1.3.1).

GCP, starker Konsens

\section{Hintergrund}

MAP ist die einzige bisher bekannte autosomal-rezessiv vererbte Erkrankung mit erhöhtem KRK-Risiko. Ob heterozygote Anlageträger ein erhöhtes KRK-Risiko tragen, wird kontrovers diskutiert; einige neuere populations- und familienbasierte Untersuchungen an großen Kollektiven fanden Hinweise für ein moderat erhöhtes Risiko im fortgeschrittenen Alter (RR 1,5 - 2,1) [267, 359, 360]. Das höchste Erkrankungsrisiko für ein KRK (RR 2,1 ) bestand bei erstgradig verwandten heterozygoten Anlageträgern eines MAP-Patienten, das Diagnosealter betrug hier im Mittel 70 Jahre (Streuung 58 -82). Erkrankungsrisiko und -zeitpunkt sind somit vergleichbar dem von erstgradig Verwandten eines Patienten mit sporadischen KRK.

Aufgrund einer Heterozygotenfrequenz von etwa $1-1,5 \%$ in der kaukasischen Allgemeinbevölkerung haben die obligat heterozygoten Kinder eines MAP-Patienten bei nicht konsanguinen Partnerschaften ein nur geringes Erkrankungsrisiko für eine MAP $(<0,5 \%)$ [262]. Wenn zur Einschätzung des Erkrankungsrisikos eine prädiktive Testung des Kindes gewünscht wird, muss beim Kind oder dem nicht erkrankten Elternteil eine komplette Mutationssuche im MUTYH-Gen erfolgen, um eine ggf. vom gesunden Elternteil vererbte zweite MUTYH-Keimbahnmutation zu identifizieren. Dem Nutzen eines aussagekräftigen genetischen Befunds steht hier allerdings die (seltene) Identifizierung funktionell unklarer genetischer Varianten gegenüber, über deren pathogene Relevanz und damit klinische Konsequenzen im Einzelfall (derzeit) keine Aussage möglich ist. Da das Vorhandensein einer zweiten MUTYH-Mutation bei Kindern eines MAP-Patienten aufgrund der zu vermutenden unvollständigen Mutationsdetektionsrate nicht gänzlich ausgeschlossen werden kann, besteht bei heterozygot getesteten Kindern eines MAP-Patienten ein - vermutlich sehr geringes - Restrisiko, an einer MAP zu erkranken. Eine komplette Koloskopie im etwa 30. und 40. Lebensjahr kann deshalb in Erwägung gezogen werden.

\subsection{Konsensbasierte Empfehlung (2013)}

Asymptomatische biallele MUTYH-Mutationsträger sollten im Alter von 18 - 20 Jahren erstmals koloskopiert werden. Finden sich keine Polypen, sollten diese Patienten weiterhin überwacht werden. Ein Patient mit einer MAP sollte in Abhängigkeit von Alter, Polypenzahl und histologischem Befund therapiert werden. Bei endoskopisch nicht beherrschbarer Polyposis ist eine Kolektomie indiziert. Patienten, die nicht kolektomiert sind, sollen zeitlebens jedes Jahr koloskopiert werden.

Eine ÖGD und Duodenoskopie (Seitblickoptik) mit besonderer Inspektion der Papillenregion soll ab dem 25. - 30. Lebensjahr mindestens alle drei Jahre durchgeführt werden. 
Spezifische Vorsorgeuntersuchungen für extraintestinale Manifestationen sind bei MAP-Patienten nicht gerechtfertigt.

Eine Empfehlung zur medikamentösen Behandlung von Adenomen im oberen und unteren Gastrointestinaltrakt kann aufgrund fehlender Daten nicht gegeben werden.

GCP, Konsens

\section{Hintergrund}

Der kolorektale Phänotyp der MAP ähnelt dem der APC-assoziierten AFAP. Bei Patienten mit einer MAP werden Polypen und KRKs meist erst zwischen dem vierten und siebten Lebensjahrzehnt symptomatisch. Etwa 50\% der Patienten haben bei Diagnose einer MAP bereits ein manifestes KRK, bei einem Drittel dieser Fälle wurden synchrone oder metachrone KRK beobachtet [361]. Die Polypen treten im gesamten Dickdarm auf, KRKs finden sich in über $50 \%$ im rechtsseitigen Kolon und in über $20 \%$ im Rektosigmoid [263]. Aus diesem Grunde muss eine komplette Koloskopie zur Früherkennung durchgeführt werden [254, 255, 257, 258]. Das Auftreten von MAP-assoziierten KRKs vor dem 29. Lebensjahr ist eine Rarität. Da die klinische Ausprägung stark variieren kann, ist die Therapieentscheidung individuell abzuwägen. Bei Patienten mit bestehender Operationsindikation und wenigen Rektumpolypen ist unter Umständen eine ileorektale Anastomose mit Belassen eines Rektumstumpfs vertretbar [362].

Obwohl eine Duodenalpolyposis bei MAP-Patienten seltener (17\%) als bei FAP-Patienten beobachtet wird, erscheint das Risiko von etwa $4 \%$ für die Entwicklung eines Duodenalkarzinoms vergleichbar hoch zu sein [269]. Bei der MAP treten Duodenalkarzinome vereinzelt auch ohne vorbestehende duodenale Adenome auf [363]. Ob für MAP-Patienten deshalb möglicherweise andere Früherkennungsstrategien als für (A)FAP-Patienten erforderlich sind, lässt sich derzeit noch nicht abschließend beurteilen. Extraintestinale Malignome treten bei MAP-Patienten insgesamt signifikant häufiger (RR 1,9\%) als in der Allgemeinbevölkerung auf und zeigen eine gewisse Überlappung zum HNPCC. In der diesbezüglich bisher einzigen systematischen Studie an $276 \mathrm{~Pa}-$ tienten wurde eine gering bis moderate, aber statistisch signifikant erhöhte Inzidenz für Ovarial-, Blasen- und Hautkarzinome sowie ein Trend zur Risikoerhöhung für Mammakarzinome beobachtet [269]. Es fanden sich allerdings kein dominierender extraintestinaler Tumor und keine Verschiebung zu einem frühen Auftreten (medianes Diagnosealter der 4 Malignome zwischen 51 und 61 Jahren). Desmoide wurden nicht beobachtet.

\subsubsection{Nicht adenomatöse Polyposis-Syndrome}

\subsection{Statement (2013)}

Hierzu zählen insbesondere die hamartomatösen Polyposis-Syndrome (Peutz-Jeghers-Syndrom, Familiäre Juvenile Polyposis, Cowden-Syndrom), das Hyperplastische Polyposis-Syndrom und die Erbliche gemischte Polyposis. Diese Erkrankungen sind z.T. sehr selten (ihr Anteil an allen KRK beträgt unter einem Promille). Anlageträger haben ein erhöhtes Risiko sowohl für kolorektale Karzinome als auch für andere syndromspezifische intestinale und extraintestinale Tumoren (Magen, Mamma etc.).

Level of Evidence 2a, Evidenz aus Aktualisierungsrecherche: [261, 364 - 394]

\section{Hintergrund}

Die Differenzialdiagnose der nicht adenomatösen Polyposis-Syndrome kann in Einzelfällen sehr schwierig sein und erfordert das interdisziplinäre Zusammenwirken von Gastroenterologen, Chirurgen, Pathologen, Humangenetikern, Radiologen und anderen klinischen Fachdisziplinen (v.a. Gynäkologie, Urologie). Die Diagnose und klinische Betreuung der Patienten sollte daher in Abstimmung und Zusammenarbeit mit Zentren erfolgen, die Erfahrung mit diesen Syndromen haben [261, 364]. Die hamartomatösen Polyposis-Syndrome folgen einem autosomal-dominanten Erbgang. Kinder (und ggf. auch Geschwister) einer erkrankten Person haben somit ein 50\%iges Risiko, die zugrunde liegende genetische Veränderung geerbt zu haben und im Laufe des Lebens zu erkranken.

Das Peutz-Jeghers-Syndrom (PJS) ist eine autosomal-dominant erbliche Erkrankung, die charakterisiert ist durch das Auftreten von hamartomatösen Polypen des Gastrointestinaltrakts und mukokutanen Melaninpigmentierungen, die vor allem perioral auffällig sind. Letztere blassen aber oft im Laufe des Lebens ab und sind nicht spezifisch. Peutz-Jeghers-Polypen treten insbesondere im Dünndarm auf und zeigen eine charakteristische Histologie. Ursächlich sind Keimbahnmutationen des STK11 / LKB1Gens. Ein Mutationsnachweis gelingt bei Patienten, die die klinisch-diagnostischen Kriterien erfüllen, in über 90\% [365]. Das Manifestationsalter ist sehr variabel, einige Patienten entwickeln schon in den ersten Lebensjahren Symptome. Zu den Komplikationen im Kindesalter zählen das akute Abdomen durch Invaginationen oder einen obstruktiven Ileus sowie die chronische Blutung mit sekundärer Anämie. Bis zu 30\% der Patienten sind im Alter von 10 Jahren bereits einmal laparotomiert worden [366]. Beim PJS muss für eine Reihe von intestinalen und extraintestinalen Tumoren von einem deutlich erhöhten Erkrankungsrisiko ausgegangen werden [367 - 373]. Neben dem KRK ist insbesondere das Risiko für Karzinome der Mamma, des Magens, des Dünndarms, des Pankreas, der Hoden, des Ovars und Uterus erhöht $[375,376]$ Das kumulative Lebenszeitrisiko für einen malignen Tumor wird mit etwa $85-90 \%$ angegeben. Für Tumoren im gesamten Gastrointestinaltrakt besteht ein kumulatives Lebenszeitrisiko von $57 \%$, das KRK-Risiko allein beläuft sich auf 35 $39 \%$ und stellt damit das zweithäufigste Karzinom bei PJS dar. Die untere Grenze des $95 \%$-Konfidenzintervalls beträgt 30 Jahre. Das Tumorrisiko steigt dabei nach dem 50. Lebensjahr rasch an [374-376]. Das Lebenszeitrisiko für gynäkologische Tumoren wird mit $13-18 \%$ angegeben [375, 376]. Ovarialtumoren bei PJS sind in der Regel SCTAT und nicht epithelialen Ursprungs und werden zum Teil bereits auch bei kleinen Mädchen diagnostiziert (mittleres Alter 28 Jahre, 4-57 Jahre). Zervixkarzinome treten mit einem Lebenszeitrisiko von $9 \%$ auf und entsprechen histologisch in mehr als drei Viertel der Fälle einem Adenoma malignum [375]. Das mittlere Erkrankungsalter beträgt 34 Jahre (23-54 Jahre). Das Risiko für Endometriumkarzinome wird mit etwa $10 \%$ angegeben.

Der Verdacht auf eine Familiäre Juvenile Polyposis (FJP) besteht bei der Diagnose von fünf oder mehr juvenilen Polypen im Kolon, Nachweis von extrakolischen juvenilen Polypen oder bei Nachweis eines juvenilen Polypen bei entsprechender positiver Familienanamnese. Die korrekte Diagnose juveniler Polypen kann 'aufgrund morphologischer Ähnlichkeiten mit hyperplastischen Polypen sowie lymphozytären Infiltraten und dysplastischen Anteilen schwierig sein: in einem nennenswerten Teil der genetisch gesicherten Fälle wird die juvenile Polyposis initial als Colitis ulcerosa oder hyperplastische Polyposis fehlgedeutet [377, 378]. Im Zweifelsfall sollte deshalb die Zweitbegutachtung der histologischen Präparate durch einen gastroenterologisch versierten Pathologen angestrebt werden.

Die Erkrankung kann sich bereits im frühen Kindesalter durch eine chronische gastrointestinale Blutung oder exsudative Ente- 
ropathie mit begleitender Entwicklungsverzögerung bemerkbar machen. Ursächlich sind Keimbahnmutationen im SMAD4- oder BMPR1A-Gen. Das Lebenszeitrisiko für die Entwicklung eines KRKs beträgt bis zu $68 \%$. Es bestehen klare Genotyp-Phänotyp-Beziehungen: Patienten mit einer SMAD4-Keimbahnmutation haben ein erhöhtes Risiko für die Entwicklung von Magenpolypen und Magenkrebs sowie für eine hereditäre hämorrhagische Teleangiektasie (Morbus Osler-Rendu-Weber) [378, 379]. Daneben ist möglicherweise das Risiko für Pankreaskarzinome erhöht [380 - 384]. Bei sehr schweren frühmanifesten Verläufen ist an die seltene juvenile Polyposis des Kleinkindesalters zu denken [385].

Die endoskopisch-histologische Abgrenzung einer juvenilen Polyposis vom auf PTEN-Mutationen beruhenden Cowden-Syndrom oder dem vermutlich nicht erblichen Cronkhite-CanadaSyndrom kann Probleme bereiten und erfolgt in der Regel durch das im Vordergrund stehende extraintestinale Tumorspektrum und die Molekulargenetik. Beim Cowden-Syndrom [386] ist insbesondere das Risiko für Mamma- und Schilddrüsenkarzinome erhöht. Das Risiko für kolorektale Karzinome scheint nach neueren Daten mit einem Lebenszeitrisiko von $28 \%$ ebenfalls erhöht zu sein [387]. Weiterhin wurden erhöhte Risiken für Karzinome des Endometriums und der Nieren sowie für Melanome beschrieben. Das Bannayan-Riley-Ruvalcaba-Syndrom wird als Variante des Cowden-Syndroms betrachtet. Beide Syndrome sind mit Keimbahnmutationen des PTEN-Gens assoziiert und werden unter der Bezeichnung PTEN-Hamartom-Tumor-Syndrom (PHTS) zusammengefasst [388, 389].

Bei der erblichen gemischten Polyposis (hereditary mixed polyposis syndrome, HMPS) und dem hyperplastischen Polyposis-Syndrom (HPS) handelt es sich um schlecht definierte Entitäten, über deren genetische Grundlagen noch wenig bekannt ist. Beide Syndrome wurden mit einem - z.T. deutlich - erhöhten KRK-Risiko in Verbindung gebracht [390-394]; sie sind jedoch selten und bisher sowohl klinisch wie genetisch schlecht charakterisiert, sodass die Erkenntnisse in Bezug auf Tumorrisiken nur bedingt aussagekräftig sind. Bei einigen Patienten mit HMPS wurden Mutationen im PTEN- oder BMPR1A-Gen nachgewiesen, diese Fälle sollten als (atypische) Varianten des Cowden-Syndroms bzw. der FJP betrachtet und entsprechend behandelt werden.

\subsection{Konsensbasierte Empfehlung (2013)}

Generelle Überwachungsempfehlungen können wegen der spärlichen Datenlage nicht gegeben werden. Die Überwachung der Patienten und Risikopersonen sollte in Zusammenarbeit mit einem ausgewiesenen Zentrum durchgeführt werden.

GCP, Konsens

\section{Hintergrund}

Valide Vorsorgeempfehlungen können wegen der spärlichen Datenlage nicht gegeben werden. Für die häufigeren Syndrome wurden von einzelnen Experten spezifische Früherkennungsprogramme vorgeschlagen. Eine publizierte Leitlinie zum Peutz-Jeghers-Syndrom hat methodische Defizite und weist für die meisten Empfehlungen nur geringe Evidenzen auf [395]. Die Patienten und Risikopersonen sollten deshalb in enger Abstimmung mit erfahrenen Zentren betreut werden. Für einige Syndrome wurden von Experten spezifische altersabhängige Früherkennungsprogramme vorgeschlagen (Übersicht in [261]), die Empfehlungen weichen allerdings zum Teil deutlich voneinander ab und die Wirksamkeit ist bei den sehr seltenen Syndromen aufgrund geringer Fallzahlen nur schwer zu validieren.

\subsection{Chronisch entzündliche Darmerkrankungen}

5.3.1. Colitis ulcerosa

5.49. Konsensbasierte Statement (2013)

Das kolorektale Karzinomrisiko ist bei Patienten mit Colitis ulcerosa im Vergleich zur Normalbevölkerung erhöht. Entsprechende Empfehlungen sind in der S3-Leitlinie zur Diagnostik und Therapie der Colitis ulcerosa genannt.

GCP, starker Konsens

Überwachungskoloskopie, zeitliche Strategie (Auszug aus der S3LL CU [396])

\subsection{Empfehlung (2013)}

Da die kolitisassoziierte Kolonkarzinommortalität durch eine endoskopische Überwachung gesenkt werden kann, sollen regelmäßige Überwachungskoloskopien erfolgen.

Empfehlungsgrad A, Evidenzgrad ${ }^{4}$ 3a, [397, 398], Konsens

\subsection{Empfehlung (2013)}

Zur Festlegung der Überwachungsstrategie soll bei allen CU-Patienten unabhängig von der Krankheitsaktivität eine Kontrollkoloskopie zur Erfassung des Befallsmusters spätestens 8 Jahre nach Beginn der Symptomatik erfolgen.

Empfehlungsgrad A, Evidenzgrad 4, [399], Konsens

\subsection{Empfehlung (2013)}

Die Überwachungskoloskopien sollten dann bei ausgedehnter $\mathrm{CU}$ ab dem 8. Jahr und bei linksseitiger oder distaler CU ab dem 15. Jahr nach Erstmanifestation 1- bis 2-jährlich erfolgen. Empfehlungsgrad B, Evidenzgrad 4, [400, 401], Konsens

\subsection{Empfehlung (2013)}

Wenn gleichzeitig eine PSC besteht, sollen die Überwachungskoloskopien unabhängig von der Krankheitsaktivität und Ausdehnung der CU ab dem Zeitpunkt der PSC-Diagnosestellung jährlich erfolgen. Empfehlungsgrad A, Evidenzgrad 3a, [402, 403], Konsens

\subsection{Empfehlung (2013)}

Nach subtotaler Kolektomie sollen in Analogie die gleichen endoskopischen Überwachungsstrategien wie bei einer CU ohne Resektion erfolgen.

Empfehlungsgrad A, Evidenzgrad 4, [404], starker Konsens

\section{Erläuterung}

Eine Metaanalyse von Collins aus dem Jahr 2006 fasst die direkte und indirekte Evidenz zu Überwachungskoloskopien bei Colitis ulcerosa zusammen. Die drei identifizierten Fallkontrollstudien konnten keine statistisch signifikante Reduktion des Risikos, an einem kolorektalen Karzinom zu erkranken, zeigen. Dabei ist anzumerken, dass es sich um kleine Studien mit teilweise aus heutiger Sicht inadäquatem Koloskopiestandard handelte. Dahingegen konnte die Metaanalyse deutliche indirekte Evidenz identifizieren, dass Überwachungskoloskopien sehr wahrscheinlich das Risiko, an einem kolitisassoziierten Kolonkarzinom zu versterben, vermindern können, und gleichzeitig kosteneffektiv sind [397]. Dies basiert darauf, dass kolitisassoziierte Kolonkarzinome früher erkannt werden, obwohl dennoch zwischen Überwachungsuntersuchungen Intervallkarzinome auftreten können

\footnotetext{
${ }^{4}$ In der Leitlinie Colitis Ulcerosa wird nicht der Level of Evidence angegeben, sondern der Evidenzgrad (396. Dignass A. et al., [Updated German guideline on diagnosis and treatment of ulcerative colitis, 2011]. Z Gastroenterol 2011; 49 (9): 1276 - 341. S. $7 f f)$
} 
[398]. Das Karzinomrisiko steigt mit der Krankheitsdauer und der Krankheitsausdehnung an, weshalb bei Patienten mit einer Pankolitis die regelmäßige Überwachung früher als bei Patienten mit einer distalen Kolitis beginnen sollte. Da sich aus einer initial als distale Kolitis beschriebenen Entzündung auch ohne klinische Hinweise eine Pankolitis entwickeln kann, sollte eine Kontrollkoloskopie innerhalb von 8 Jahren nach den ersten Krankheitssymptomen durchgeführt werden, um die Ausdehnung zu überprüfen und danach das Überwachungsvorgehen festlegen zu können. Eine niederländische Studie weist darauf hin, dass bereits bis zu 22 \% der Patienten vor dem Start der bisher empfohlenen Überwachungskoloskopien kolitisassoziierte Kolonkarzinome entwickelten [399]. Wenn Patienten mit PSC herausgerechnet wurden - die ab Diagnosestellung überwacht werden sollten - reduziert sich die Rate der „entgangenen“ Karzinome auf ca. $15 \%$.

Das Überwachungsintervall zwischen den Untersuchungen sollte höchstens 2 Jahre betragen, weil in diesem Zeitraum bereits Intervallkarzinome auftreten können [400, 401]. Da bei einer Proktitis das Karzinomrisiko allenfalls minimal erhöht ist, muss - bei Fehlen sonstiger Risikofaktoren - keine regelhafte Überwachung erfolgen. Da das Karzinomrisiko bei Patienten mit CU und einer
PSC 5-fach erhöht ist [403] und Karzinome gehäuft rechtsseitig auftreten [402], sollte bei diesen Patienten eine Überwachung ab Diagnosestellung unabhängig vom Befall jährlich erfolgen. Nach subtotaler Kolektomie können im verbliebenen Darm, wie auch nach restaurativer Proktokolektomie im Pouch bzw. je nach Operationstechnik im Bereich der verbliebenen Kolonmukosa distal der Anastomose, Karzinome auftreten [404], sodass eine regelhafte Überwachung des verbliebenen Kolons bzw. des Pouches erfolgen sollte.

\subsubsection{Morbus Crohn}

Das kolorektale Karzinomrisiko bei Patienten mit Morbus Crohn scheint, insbesondere bei Kolonbefall, im Vergleich zur Normalbevölkerung erhöht zu sein. Der Nutzen eines Überwachungsprogramms mit Ileokoloskopie zur Früherkennung eines Karzinoms ist bei der Crohn Colitis ungeklärt. Die Datenlage wird in der S3-Leitlinie zur Diagnostik und Therapie des Morbus Crohn diskutiert [405].

\subsection{Anhang: Abbildungen und Tabellen des TK III}

5.4.1. Algorithmus: Genetische Diagnostik und Vorsorge (o Abb. 2)

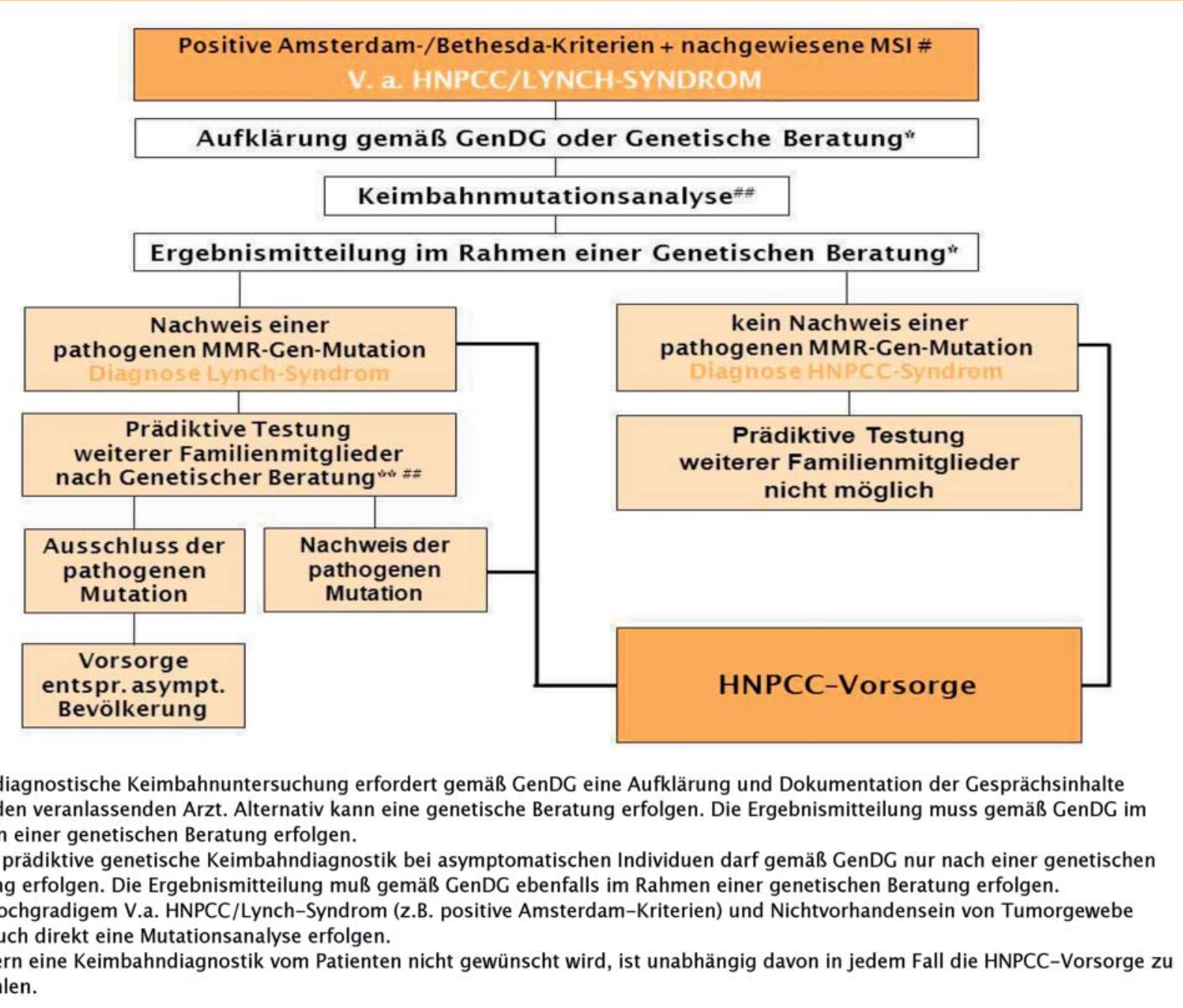

Abb. 2 Algorithmus zum Ablauf der genetischen Diagnostik bei Patienten mit V. a. ein hereditäres Tumordispositions-Syndrom am Beispiel des HNPCC/LynchSyndroms. Zum Nachweis der MSI bei V. a. HNPCC/Lynch-Syndroms wird auf Abb. 3 verwiesen. 


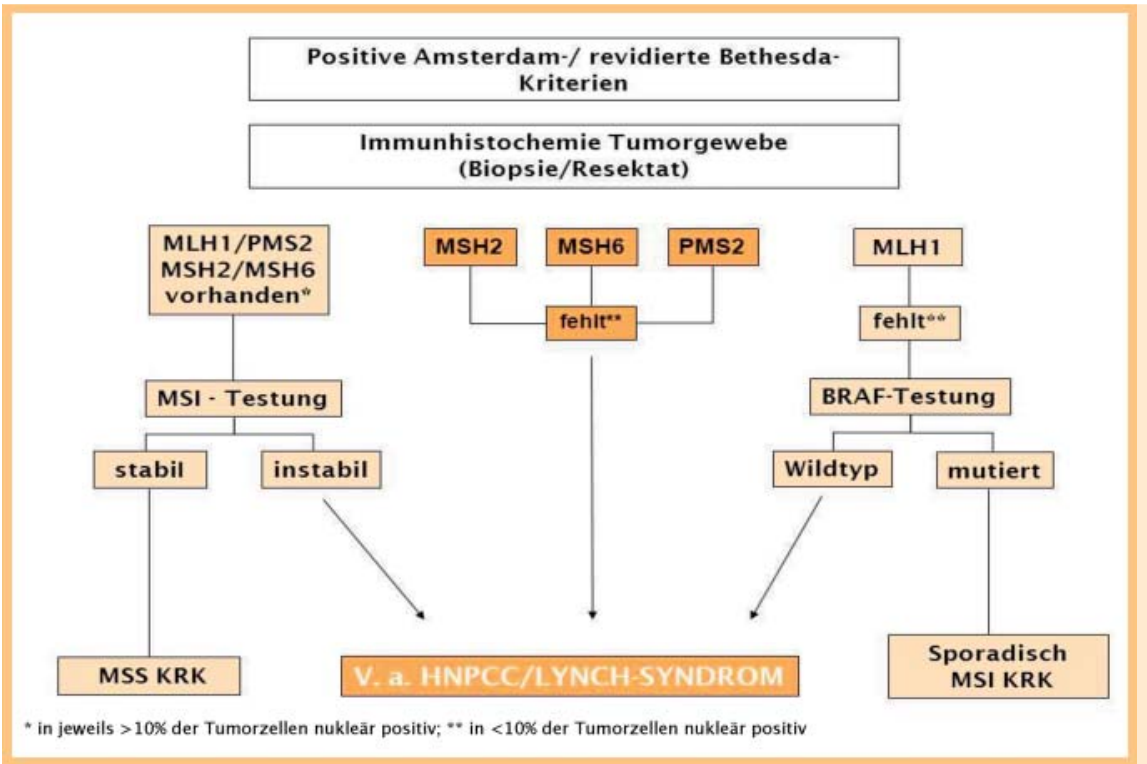

Abb.3 Algorithmus zum Ablauf der molekularpathologischen Abklärung eines Mismatch-Reparaturdefekts bei klinischem V. a. HNPCC/Lynch-Syndrom. Zur sich ggf. anschließenden genetischen Diagnostik wird auf Abb. 2 verwiesen.

\subsubsection{Empfohlenes Krebsfrüherkennungsprogramm bei HNPCC (o Tab. 6)}

Tab.6 Empfohlenes Krebsfrüherkennungsprogramm bei HNPCC.

\begin{tabular}{|c|c|c|}
\hline Altersangabe & Untersuchung & Intervall \\
\hline \multirow{3}{*}{$\begin{array}{l}\text { Ab dem } 25 . \\
\text { Lebensjahr }\end{array}$} & Körperliche Untersuchung & jährlich \\
\hline & Koloskopie & jährlich \\
\hline & $\begin{array}{l}\text { Gynäkologische Untersuchung einschl. } \\
\text { transvaginaler Sonografie (bei Frauen) }\end{array}$ & jährlich \\
\hline \multirow{2}{*}{$\begin{array}{l}\text { Ab dem } 35 . \\
\text { Lebensjahr }\end{array}$} & ÖGD & regelmäßig \\
\hline & Endometriumbiopsie (bei Frauen) & jährlich \\
\hline
\end{tabular}

\subsubsection{Spigelman-Klassifikation (o Tab.7)}

Tab. 7 Klassifikation der Ausprägung der Duodenalpolyposis nach der Spigelman-Klassifikation (modifiziert nach [307]).

\begin{tabular}{|c|c|c|c|}
\hline & \multicolumn{3}{|l|}{ Punktezahl } \\
\hline & 1 & 2 & 3 \\
\hline $\begin{array}{l}\text { Anzahl der } \\
\text { Polypen }\end{array}$ & $1-4$ & $5-10$ & $>20$ \\
\hline $\begin{array}{l}\text { Polypengröße } \\
(\mathrm{mm})\end{array}$ & $1-4$ & $5-20$ & $>10$ \\
\hline Histologie & tubulär & tubulär villös & villös \\
\hline $\begin{array}{l}\text { Intraepitheliale } \\
\text { Neoplasie }\end{array}$ & niedriggradig & - & hochgradig \\
\hline $\begin{array}{l}\text { Stadium 0: } 0 \text { Pur } \\
\text { Stadium I: } 1 \text { - } 4 \\
\text { Stadium II: } 5-6 \\
\text { Stadium III: } 7-8 \\
\text { Stadium IV: } 9-1\end{array}$ & $\begin{array}{l}\text { e } \\
\text { ikte } \\
\text { nkte } \\
\text { Inkte } \\
\text { unkte }\end{array}$ & & \\
\hline
\end{tabular}

\section{Endoskopie: Durchführung und Polypenmanagement \\ $\nabla$}

6.1. Stellenwert der Endoskopie in der Diagnostik von Polypen und kolorektalen Karzinomen

6.1. Statement (2008)

Die komplette Koloskopie stellt das Standardverfahren zur Detektion kolorektaler Polypen und Karzinome dar. Sie besitzt die höchste Sensitivität und Spezifität für das Auffinden eines KRK und von kolorektalen Polypen. Die Effektivität der Koloskopie hängt entscheidend von der Qualität der Untersuchung ab. Diese ist technikund untersucherabhängig.

Level of Evidence 1b, starker Konsens

\subsection{Empfehlung (2008)}

Bei inkompletter Koloskopie aufgrund eines stenosierenden Tumors kann präoperativ zusätzlich eine CT- oder MR-Kolonografie erfolgen. Postoperativ soll eine komplette Koloskopie erfolgen. Empfehlungsgrad A, Level of Evidence 4, starker Konsens

\subsection{Empfehlung (2008)}

Bei inkompletter Koloskopie infolge anderer Ursachen (z. B. Adhäsionen) sollte eine CT- oder MR-Kolonografie erfolgen. Empfehlungsgrad B, Level of Evidence 4, starker Konsens

\section{Hintergrund}

Die Koloskopie ist das zuverlässigste Verfahren zur Detektion kolorektaler Karzinome und Polypen, wenn sie mit hoher Qualität durchgeführt wird. Wichtige Qualitätsmerkmale beinhalten die Spiegelung bis zum Coecum, die optimale Darmvorbereitung mit wenig oder keinen verbliebenen Stuhlresten sowie die sorgfältige Inspektion der Darmschleimhaut beim Rückzug. So konnte gezeigt werden, dass die Polypendetektionsrate mit der Rückzugszeit nach Erreichen des Coecums korreliert. Die Rückzugszeit sollte mindestens 6 min betragen [406 - 408]. Weitere Qualitätsmerkmale sind die primäre Erkennung von Polypen bei $20-50 \%$ 
(polyp detection rate) [409] sowie übersehene Polypen bei weniger als $10 \%$ der Untersuchten (polyp miss rate) [410, 411].

Allerdings hat die Koloskopie auch Limitationen: So werden 4 $6 \%$ der Karzinome übersehen [412-414]. Auch treten bei der Polypennachsorge in einem Zeitraum von 3 Jahren bis zu $1 \%$ Intervallkarzinome auf [415]. Diese sind z.T. auf übersehene Läsionen zurückzuführen (s. auch Abschnitt Polypenmanagement, Kapitel 6.5). Sensitivität und Spezifität der CT-Kolonografie sind in den letzten Jahren seit Erstellung der letzten Leitlinie angestiegen. In einer Studie waren Koloskopie und CT-Kolonografie bei der Entdeckung von Polypen $>5 \mathrm{~mm}$ gleichwertig [410]. In anderen Untersuchungen konnte dieses besonders gute Ergebnis jedoch nicht erzielt werden [416, 417]. Hier war die CT-Kolonografie der Koloskopie zum Teil deutlich unterlegen $[418,419]$, der Röntgendoppelkontrastuntersuchung jedoch überlegen [417, 420]. Die Röntgendoppelkontrastuntersuchung des Dickdarms ist heute obsolet. Für die Beurteilung der MR-Kolonografie liegen noch zu wenige Studienergebnisse vor [421-423].

Diagnostische Probleme bei der CT-Kolonografie können Stuhlreste und eine schlechte Entfaltung des Darmlumens bereiten. Die Detektion flacher, eingesenkter und kleiner Polypen ist schwieriger als die prominenter Polypen. Aufgrund mangelnder Standardisierung sind die Ergebnisse derzeit stark zentrenabhängig. Hinzu kommen der hohe Preis und die mit der CT-Kolonografie verbundene Strahlenbelastung.

Aus diesen Gründen ist bei einem positiven fäkalen Okkultbluttest (FOBT) oder zur Abklärung eines Tumorverdachts die komplette Koloskopie der Goldstandard. Es besteht hierbei die Möglichkeit sowohl der Biopsieentnahme zur histologischen Diagnostik als auch der Polypektomie als gleichzeitige therapeutische Intervention.

Bei pathologischen Befunden im Rahmen der Koloskopie ist eine Zuordnung nach endoskopisch-anatomischen Strukturen und nach Diaphanoskopie ungenügend, eine Angabe in cm Gerätelänge ab ano sollte nur im Rektum und unteren Sigma erfolgen. Bei unklarem oder OP-würdigem Befund sollte eine Markierung mittels Clip (nur bei zeitnaher OP) oder Tusche erfolgen, um eine Wiederauffindung zu ermöglichen.

Bei Patienten mit stenosierenden Tumoren oder inkompletter Koloskopie aus anderen Gründen konnten in Fallserien mittels CT- oder MR-Kolonografie proximale Tumoren oder Polypen nachgewiesen werden [424-427].

\subsubsection{Sigmoidoskopie versus Koloskopie 6.4. Empfehlung (2008)}

Bei positivem FOBT-Test, bei Tumorverdacht oder sigmoidoskopischem Nachweis eines neoplastischen Polypen soll eine vollständige Koloskopie durchgeführt werden.

Empfehlungsgrad A, Level of Evidence 2b, starker Konsens

\section{Hintergrund}

Zur Abklärung eines positiven FOBT-Testes bzw. eines Tumorverdachts wird die komplette Koloskopie gefordert, da hiermit auch Adenome und Karzinome im rechten Hemikolon erkannt werden können. Relevante neoplastische Läsionen proximal des Sigmas findet man in 25 - 55\%. In Screening-Studien konnte gezeigt werden, dass bei 30 bis $46 \%$ der Fälle mit proximalen fortgeschrittenen Neoplasien im rechten Hemikolon das Rektosigmoid adenomfrei ist $[107,127]$.

Eine Sigmoidoskopie sollte nur im Ausnahmefall erfolgen, wenn eine vollständige Darmvorbereitung nicht möglich ist. Eine kom- plette Koloskopie ist in einem hohen Prozentsatz möglich und kann auch bei älteren Patienten in der Regel mit einer niedrigeren Rate an Nebenwirkungen durchgeführt werden [428, 429]. Die Sigmoidoskopie hat sowohl als Suchmethode wie auch für die Primärdiagnostik in Deutschland keine Bedeutung.

\subsubsection{Chromoendoskopie}

\subsection{Empfehlung (2008)}

Eine Chromoendoskopie kann bei Patienten mit einer chronisch entzündlichen Darmerkrankung und HNPCC zur besseren Erkennung von neoplastischen Läsionen eingesetzt werden. Sie kann darüber hinaus zur besseren Abgrenzung flacher und eingesenkter Läsionen vor endoskopischer Therapie verwendet werden. Empfehlungsgrad 0, Level of Evidence 1b, starker Konsens

\section{Hintergrund}

Bei Patienten mit CED oder HNPCC kann eine höhere Detektionsrate neoplastischer Läsionen durch Chromoendoskopie als gesichert gelten [430 - 432]. In Studien an Patienten ohne hereditäre Tumorerkrankungen oder CED fanden sich ebenfalls höhere Raten vorwiegend kleiner Adenome [433-435], die allerdings in einer weiteren Studie nicht bestätigt wurden [436].

Es ist bisher nicht geklärt, ob die Erkennung höherer Raten vorwiegend kleiner Veränderungen dem Patienten nutzen und den erhöhten Zeitaufwand rechtfertigen.

Durch den Einsatz der Chromoendoskopie mit Indigokarmin oder Methylenblau gelingt eine bessere Abgrenzung flacher und eingesenkter Läsionen von der umgebenden gesunden Schleimhaut [437 - 442]. Die Chromoendoskopie kann daher vor der endoskopischen Abtragung flacher Adenome eingesetzt werden.

\subsubsection{Zoomendoskopie \\ 6.6. Empfehlung (2008)}

Die Durchführung der Zoomendoskopie zur Klassifikation des „Pitpattern"-Musters ist derzeit kein Standardverfahren.

Empfehlungsgrad 0, Level of Evidence 2b, starker Konsens

\section{Hintergrund}

Ziel der Zoomendoskopie ist es, anhand der „Pit-pattern“-Klassifikation zwischen hyperplastischen und neoplastischen Läsionen zu unterscheiden, um ohne Histologie festzustellen, welche Läsionen endoskopisch abgetragen werden müssen. In einzelnen Studien war dies mit hoher Sensitivität möglich [434, 443, 444]. Die Spezifität war jedoch in anderen Studien mit $75 \%$ nicht ausreichend [439, 441, 445, 446]. Die Zoomendoskopie ersetzt derzeit nicht die Histologie. Die Zoomendoskopie ist in der Einschätzung polypoider und flacher Läsionen vor Polypektomie nach bisher vorliegenden Ergebnissen hilfreich, kann aber derzeit nicht als Standard empfohlen werden.

Verfahren wie das Narrow-band imaging (NBI) oder Fuji intelligent chromendoscopy (FICE) stellen weitere Methoden zur Abgrenzung neoplastischer Läsionen dar. In unkontrollierten Studien war das NBI hilfreich in der Detektion flacher Adenome, der Detektion intraepithelialer Neoplasien bei Colitis ulcerosa sowie der Dignitätsbeurteilung [447-449]. Für FICE liegen bisher keine vergleichbaren Daten vor. Die konfokale Lasermikroskopie bleibt derzeit noch wissenschaftlichen Fragestellungen vorbehalten [450]. 


\subsection{Polypektomiedurchführung}

6.2.1. Schlingenektomie versus Zangenektomie

6.7. Empfehlung (2008)

Polypen sollen unter Angabe der Lokalisation entfernt und geborgen werden. Die Polypektomie kann bei multiplen Polypen ggf. mehrzeitig erfolgen.

Empfehlungsgrad A, Level of Evidence 1c, starker Konsens

\section{Hintergrund}

Um eine Zuordnung zu ermöglichen, sollen die Polypen einzeln unter Angabe der Lokalisation für eine histologische Aufarbeitung geborgen werden. Bei mehreren Polypen in einem Segment ist eine gemeinsame Bergung dieser Polypen vertretbar. Hierbei müssen aber die onkologischen Resektionsgrenzen beachtet werden; eine Markierung des polypektomierten Kolonsegments ist sinnvoll.

Folgende endoskopische Verfahren stehen zur Verfügung:

- Polypektomie mit der Schlinge

- Endoskopische Mukosaresektion (EMR)

Alternative Verfahren der Polypenentfernung (offene oder laparoskopische Resektion, Rendezvousverfahren, TEM, transanale Abtragung) sind im Einzelfall in Erwägung zu ziehen. Neuere Therapieverfahren wie die endoskopische Submukosadissektion (ESD) zur Erreichung einer en-bloc-Resektion sind derzeit in Erprobung und noch wissenschaftlichen Fragestellungen vorbehalten. Abgetragene flache und sessile Polypen sollten zur Identifikation durch Aufspießen mit einer Stecknadel oder durch Farbstoff markiert werden. Die Fixierung auf einer Korkplatte hat sich ebenfalls bewährt.

Voraussetzung und Limitierung für die endoskopische Schlingenektomie großer Polypen ist die realistische Option einer kompletten Abtragung mit einem niedrigen Blutungs- und Perforationsrisiko. Die Erfahrung des Untersuchers und die Lage des Polypen können ebenfalls limitierende Faktoren darstellen. $\mathrm{Zu}$ bedenken sind die mit der Größe ansteigende Karzinomrate neoplastischer Polypen (bis zu 15\%), die Wuchsform, die Lokalisation, der Allgemeinzustand des Patienten sowie das erhöhte Perforationsrisiko im proximalen Kolon [451 - 455]. Flache Läsionen können durch eine endoskopische Mukosektomie (EMR) entfernt werden [455 - 457]. Ausschließlich eingesenkte flache Läsionen (IIc) sollten in der Regel primär chirurgisch und nicht endoskopisch behandelt werden, da es sich hierbei meistens nicht mehr um sogenannte frühinvasive T1-Karzinome handelt und die komplette endoskopische Entfernung (R0) nur selten möglich ist. Einen Hinweis liefert eine fehlende Abhebbarkeit (non-lifting sign). Unabhängige Faktoren bezüglich des Perforationsrisikos sind Polypengrößen über $1 \mathrm{~cm}$ sowie die Lokalisation im rechten Kolon, bez. des Blutungsrisikos lediglich die Polypengröße $>1 \mathrm{~cm}$ [458]. Das Risiko schwerer Blutungen (Transfusionspflichtigkeit, Operationsnotwendigkeit, rezidivierende Nachblutung) beträgt $0,9 \%$; das Perforationsrisiko im rechten Kolon 1,2\%, im linken Kolon $0,4 \%$. Eine prophylaktische Injektion von Kochsalz oder Adrenalin in Basis oder Stiel des Polypen oder die Verwendung von Endoloop reduziert früh auftretende Blutungen bei der Abtragung von größeren Polypen $>1 \mathrm{~cm}$ [459-462]. Die Rate verzögert auftretender Blutungen wird jedoch nicht gesenkt [463].

Die Polypektomie kann auch unter Thrombozytenaggregationshemmung durchgeführt werden [464-466], die Kombination von Acetylsalicylsäure und Clopidrogel erhöht jedoch das Blutungsrisiko und sollte vermieden werden [467]. Die vollständige Entfernung eines Polypen ist immer zu fordern, denn in einem verbliebenen Polypenrest können noch eine hochgradige intra- epitheliale Neoplasie oder ein Karzinom nachweisbar sein. Die Größe des entfernten Polypen, der histologische Adenomtyp und der Schweregrad der intraepithelialen Neoplasie bestimmen die Höhe des Risikos für Lokalrezidive und metachroner Polypen. Bei Polypen $>2 \mathrm{~cm}$ beträgt die Lokalrezidivrate $8-20 \%$ [468]. Diese Rezidive können jedoch in der Regel endoskopisch erneut entfernt werden.

Bei möglicher oder zu erwartender Operationspflichtigkeit ist eine präoperative Markierung des Polypenareals mit Clip oder Tusche obligat (Ausnahme: Zökum und distales Rektum). Eine Alternative ist die intraoperative Koloskopie zur Lokalisation des Polypen bzw. dessen Abtragungsstelle. Eine Markierung der Polypektomiestelle sollte bei schwieriger Lokalisation (erschwerte Wiederauffindbarkeit bei Überwachungsuntersuchungen) auch nach endoskopischer Intervention erfolgen.

\subsection{Empfehlung (2008)}

Um eine repräsentative histologische Aussage zu erhalten und zur definitiven Therapie, sollen Polypen $>5 \mathrm{~mm}$ vollständig durch Schlingenektomie entfernt werden. Polypen $\leq 5 \mathrm{~mm}$ sollten generell mit der Zange komplett entfernt werden.

Grundsätzlich sollen diagnostische Koloskopien nur dann durchgeführt werden, wenn in gleicher Sitzung die Möglichkeit zur Schlingenektomie besteht.

Empfehlungsgrad A, Level of Evidence 3b, starker Konsens

\section{Hintergrund}

Um Doppeluntersuchungen zu vermeiden, sollte eine Koloskopie grundsätzlich nur bei vorhandener Interventionsmöglichkeit erfolgen. Ist die Abtragung eines Befundes nicht möglich oder sinnvoll (Risikosituation in ambulanter Praxis, fehlende Expertise bei größeren Polypen), sollte der Befund ggf. markiert und der Patient in ein Zentrum überwiesen werden. Eine Zangenbiopsie von Polypen ist nicht sinnvoll, wenn eine Abtragung technisch möglich ist. Sie ist außerdem unzuverlässig [469]. Darüber hinaus können ausgiebige Biopsien durch nachfolgende Vernarbung die komplette endoskopische Abtragung beim Zweiteingriff erschweren. Bei eindeutigen Malignitätskriterien mit primärer OP-Indikation sind Biopsieentnahmen obligat. Bei Polypen $\leq 5 \mathrm{~mm}$ sind Adenome mit invasivem Karzinom äußerst selten, bei Polypen $\leq 1 \mathrm{~cm}$ beträgt die Rate $<1 \%$. Ziel einer Koloskopie muss das Erreichen eines polypenfreien Darmes (clean-colon) sein. Bei Polypen $\leq 5 \mathrm{~mm}$ ist deshalb eine komplette Zangenektomie erforderlich, um die Läsion histologisch zu typisieren. Kleine $(\leq 5 \mathrm{~mm})$ häufig multipel auftretende typische hyperplastische Polypen im Rektum können belassen werden. In den letzten Jahren mehren sich die Hinweise, dass neben der Adenom-Karzinom-Sequenz auch der weitere Weg über den sog. „serrated pathway“ zu einem kolorektalen Karzinom führen kann (s. u.).

Beim Vorliegen einer sog. Hyperplastischen Polyposis ist das mutmaßlich erhöhte Risiko eines kolorektalen Karzinoms bei der Terminierung von Kontroll-Intervallen zu berücksichtigen.

Die hyperplastische Polyposis ist definiert durch (WHO):

- mindestens 5 hyperplastische Polypen proximal des Sigmas, wobei $2<1 \mathrm{~cm}$ sein sollen;

- das Auftreten von hyperplastischen Polypen proximal des Sigmas unabhängig von Zahl und Größe, wenn ein erstgradig Verwandter (Eltern, Kinder, Geschwister) von einer hyperplastischen Polyposis betroffen ist;

- wenn mehr als 30 hyperplastische Polypen - egal welcher Größe - proximal des Sigmas auftreten [470 - 476]. 


\subsection{Histologische Untersuchung}

\subsection{Empfehlung (2008)}

Die histologische Untersuchung jedes Polypen ist obligat. Die histologische Befundung der Polypen erfolgt entsprechend der WHOKriterien [477] mit einer Aussage zur Vollständigkeit der Abtragung. Konventionelle Adenome werden klassifiziert nach histologischem Wachstumstyp (tubulär, tubulovillös und villös) und dem Grad der intraepithelialen Neoplasie (niedrig- und hochgradige intraepitheliale Neoplasie); serratierte Läsionen werden unterteilt in hyperplastische Polypen, sessile serratierte Adenome, gemischte Polypen (mit Angabe des IEN-Grades) und traditionelle serratierte Adenome (mit Angabe des IEN-Grades) [478, 479].

Empfehlungsgrad A, Level of Evidence 3b, starker Konsens

\section{Hintergrund}

Etwa $8 \%$ der bisher als hyperplastisch klassifizierten Polypen sind nach neuen Erkenntnissen sessile serratierte Adenome (SSA), die ein Progressionspotential zum Karzinom besitzen, vor allem bei einer Größe von mehr als $1 \mathrm{~cm}$ und rechtsseitiger Lokalisation. Außerdem kommen gemischte Schleimhautpolypen (mixed polyps) vor. Weiterhin sind $2 \%$ aller kolorektalen Polypen traditionelle serratierte Adenome (TSA). Alle diese Varianten weisen einen gemeinsamen molekularen Kanzerogeneseweg auf.

\subsection{Empfehlung (2008)}

Bei Karzinomnachweis soll der histologische Befund folgende Merkmale enthalten[480]:

- das Ausmaß der Tiefeninfiltration (pT-Kategorie), bei sessilen Polypen die sm-Invasionsmessung in $\mu \mathrm{m}$,

- den histologischen Differenzierungsgrad (Grading),

- Vorhandensein oder Fehlen von Lymphgefäßinvasion (L-Klassifikation),

- und die Beurteilung der Resektionsränder (R-Klassifikation) im Hinblick auf die lokale Entfernung im Gesunden (zur Tiefe und zur Seite).

Empfehlungsgrad A, Level of Evidence 2b, starker Konsens

\subsection{Empfehlung (2008)}

Im Hinblick auf weitere therapeutische Konsequenzen bei komplett entfernten pT1-Karzinomen soll eine zusammenfassende Klassifikation in „Low-risk“ (G1, G2 und keine Lymphgefäßeinbrüche [LO]) oder „High-risk“ (G3, G4, und/oder Lymphgefäßeinbrüche [L1]) erfolgen.

Empfehlungsgrad A, Level of Evidence 2b, starker Konsens

\section{Hintergrund}

Die Notwendigkeit einer Angabe über den Abstand der Entfernung im Gesunden bei pT1 Karzinomen ist strittig.

\subsection{Vorgehen bei pT1-Karzinomen}

\subsection{Empfehlung (2008)}

Ergibt die histologische Untersuchung eines endoskopisch R0-entfernten Polypen ein pT1-Karzinom, soll auf eine onkologische Nachresektion verzichtet werden, wenn es sich um eine Low-risk-Situation bei histologisch karzinomfreier Polypenbasis (RO) handelt [481 - 484]. In der High-risk-Situation ist die radikale chirurgische Behandlung erforderlich, auch wenn die Läsion komplett entfernt wurde.

Empfehlungsgrad A, Level of Evidence 3a, Konsens

\subsection{Empfehlung (2008)}

Bei inkompletter Abtragung eines Low-risk-pT1-Karzinoms soll eine komplette endoskopische oder lokale chirurgische Entfernung erfolgen [485]. Wenn eine R0-Situation nicht erreichbar ist oder Zweifel am Vorliegen einer pT1-Situation bestehen, so ist die onkologisch-chirurgische Resektion erforderlich.

Empfehlungsgrad A, Level of Evidence 3a, starker Konsens

\section{Hintergrund}

pT1-Karzinome unterscheiden sich je nach Situation erheblich in ihrer Prognose. Dreh- und Angelpunkt einer Risikostratifizierung stellt die Wahrscheinlichkeit der Lymphknotenmetastasierung dar. Die Gesamtgruppe der T1-Karzinome hat eine Lymphknotenmetastasierungsrate $(\mathrm{N}+)$ von $0-20 \%$ [482, $486-488]$. Für die Abschätzung der Metastasierungsrate gibt es qualitative und quantitative Prognosekriterien [488, 489].

Qualitative Kriterien sind: Das Grading (G1 gut differenziert, G2 mäßig differenziert, G3 schlecht differenziert, G4 undifferenziert) und die Invasion in die Lymphgefäße (L-Klassifikation) des Polypen. Quantitativ kann die Submukosainvasion am Operations- bzw. Polypektomiepräparat ausgemessen werden. Hierbei hat sich bei Operationspräparaten bewährt, die Submukosaschicht in drei Drittel aufzuteilen. Am Präparat eines sessilen Polypen, das mittels endoskopischer Polypektomie gewonnen wurde, ist die Messung der Submukosainvasionsstrecke in $\mu \mathrm{m}$ sinnvoller, weil die Submukosa als Gesamtschicht nicht zur Verfügung steht bzw. keine Muskularis propria miterfasst ist. Die sogenannten frühinvasiven Formen (sm1, sm2 bzw. Submukosainvasion $\leq 1000 \mu \mathrm{m}$ ) haben mit $0-6 \%$ ein geringes $\mathrm{N}+$-Risiko [489, 490]. Bei sm3-Karzinomen hingegen beträgt das Lymphknotenmetastasierungsrisiko etwa 20\% [490, 491]. Cave: Die Messung der Submukosadicke am gestielten Polypen in $\mu \mathrm{m}$ ist nicht sinnvoll bzw. irreführend, weil die Submukosadicke von der Stiellänge abhängig ist, d. h. der Stiel ist immer sm1-Niveau. Der Nachweis einer Veneninvasion (V-Klassifikation) sollte erwähnt werden, der Stellenwert für eine lokale Therapie ist jedoch nicht sicher belegt.

Ergänzende wichtige Anmerkungen:

In der Regel erfolgt die endoskopische Karzinomtherapie im Rahmen der Polypektomie, ohne dass vorher die Krebsdiagnose bekannt ist. Vorsicht ist geboten, wenn bei sessilen Läsionen prätherapeutisch bereits eine Karzinomdiagnose bioptisch gesichert wurde. Häufig handelt es sich dann schon nicht mehr um eine endoskopisch therapierbare Situation. Endoskopische Warnkriterien sind: Ulzerationen, Einsenkungen, Kontaktblutungen und fehlendes Lifting-sign beim Unterspritzen der Läsion. Die Absicherung einer R0-Situation ist obligat, kontrovers wird ein Sicherheitsabstand von $1 \mathrm{~mm}$ zur Basis diskutiert [489]. Eine endoskopische Entfernung als en bloc Resektion ist optimal. Eine Entfernung in Piecemeal-Technik erscheint ausreichend. Hierbei erfolgt die Beurteilung der R-Situation zur Seite endoskopisch-makroskopisch, die Beurteilung zur Tiefe histologisch (basal R0). Allerdings ist dann eine zeitnahe (2-6 Monate) endoskopisch-bioptische Überprüfung der R0-Situation erforderlich. Nach den vorliegenden Daten [482, 484, 488 - 490, 492] beträgt die postinterventionelle Rate von Metastasen bzw. Lokalrezidiven in der Low-risk-Situation $0-5 \%$, wobei das Konzept der früh invasiven Submukosakarzinome nur in die neueren Studien eingegangen ist. Die lokale endoskopische Therapie von frühen kolorektalen malignen Neoplasien ist in speziellen Zentren eine sichere und effektive Therapie und gilt bei Beachtung der Lowrisk-Kriterien als Standardtherapie. In dieser Situation ist eine 
nachfolgende radikal-chirurgische Resektion nach onkologischen Kriterien nicht erforderlich.

\subsection{Empfehlung (2008)}

Die endoskopische lokale Nachsorge soll nach kompletter Entfernung (R0) von Low-risk(pT1, low grade [G1, G2, L0])-Karzinomen nach einem halben Jahr und nach zwei Jahren erfolgen.

Empfehlungsgrad A, Level of Evidence 4, starker Konsens

\section{Hintergrund}

Die o.g. Empfehlungen dienen zur Erkennung von Lokalrezidiven. Eine komplette Koloskopie sollte zur frühzeitigen Erkennung und Sanierung von Rezidiven entsprechend den Empfehlungen der Adenomnachsorge erfolgen.

\subsection{Polypenmanagement (Nachsorge) \\ 6.15. Empfehlung (2008)}

Nach Abtragung kleiner einzelner nicht neoplastischer Polypen besteht keine Notwendigkeit einer endoskopischen Nachsorge.

Empfehlungsgrad B, Level of Evidence. 3b, starker Konsens

\section{Hintergrund}

Bei Patienten mit kleinen $(<1 \mathrm{~cm})$ hyperplastischen Polypen und negativer Familienanamnese besteht offenbar kein erhöhtes Risiko für das Auftreten eines kolorektalen Karzinoms. Hier gelten die allgemeinen Regeln zur KRK-Prävention, das heißt Kontrollkoloskopie nach 10 Jahren [493-495]. Ausnahmen sind nichtneoplastische Polyposiserkrankungen (hyperplastische, juvenile, PeutzJeghers) mit erhöhtem Risiko einer malignen Entartung [496].

\subsection{Empfehlung (2008)}

Nach kompletter Abtragung neoplastischer Polypen (Adenome) ist eine Kontrollendoskopie erforderlich. Der Zeitpunkt der Kontrollkoloskopie sollte von Anzahl, Größe und Histologie der entfernten Adenome abhängig gemacht werden. Bei Patienten mit 1 oder 2 Adenomen $<1 \mathrm{~cm}$ ohne höhergradige intraepitheliale Neoplasie ist eine Kontrollkoloskopie nach 5 Jahren ausreichend.

Empfehlungsgrad B, Level of Evidence 2b, starker Konsens

\subsection{Empfehlung (2008)}

Bei Patienten mit 3-10 Adenomen, oder mindestens einem Adenom, das $1 \mathrm{~cm}$ oder größer ist, oder einem Adenom mit villöser Histologie sollte die erste Kontrollkoloskopie nach 3 Jahren erfolgen. Empfehlungsgrad B, Level of Evidence 1b, starker Konsens

\subsection{Empfehlung (2008)}

Bei Patienten mit Adenom mit hochgradiger intraepithelialer Neoplasie und histologisch bestätigter vollständiger Abtragung ist eine Kontrollkoloskopie nach 3 Jahren ausreichend.

Empfehlungsgrad B, Level of Evidence 1b, starker Konsens

\subsection{Empfehlung (2008)}

Bei histologisch nicht bestätigter vollständiger Abtragung muss auch bei makroskopisch kompletter Abtragung eine zeitnahe (2 - 6 Monate) Kontrolle erfolgen.

Empfehlungsgrad B, Level of Evidence 3b, starker Konsens

\subsection{Empfehlung (2008)}

Bei Nachweis von mehr als 10 Adenomen sollte das Kontrollintervall kürzer als 3 Jahre sein und unter Berücksichtigung individueller Kriterien (Familienanamnese) festgelegt werden. Empfehlungsgrad B, Level of Evidence 3b, starker Konsens

\subsection{Empfehlung (2008)}

Nach Abtragung großer, flacher oder sessiler Adenome in Piecemeal-Technik muss eine kurzfristige Kontrolle der Abtragungsstelle nach 2-6 Monaten erfolgen.

Empfehlungsgrad B, Level of Evidence 3b, starker Konsens

\subsection{Empfehlung (2008)}

Nach unauffälliger Kontrollendoskopie sind weitere Kontrollen in 5-jährigen Abständen angezeigt. Nach kompletter Abtragung eines traditionellen serratierten Adenoms, eines gemischten Schleimhautpolypen oder eines sessilen serratierten Adenoms sollte aufgrund des potenziell erhöhten Karzinomrisikos unabhängig vom IEN-Grad eine Kontrollkoloskopie nach 3 Jahren erfolgen.

Empfehlungsgrad B, Level of Evidence 4, starker Konsens

\section{Hintergrund}

Die Empfehlungen zum Post-Polypektomie-Management sollten durch das individuelle Risiko des jeweiligen Patienten (familiäre Belastung, Komorbiditäten, Zweittumor, Divertikulose [497]) und den Sauberkeitsgrad des Kolons bei der zuletzt durchgeführten Koloskopie beeinflusst werden. Grundlage für die oben genannten Empfehlungen ist die Durchführung einer hochqualitativen Basiskoloskopie (s. o.). Dennoch treten Intervall-Karzinome mit einer Rate von 0,7 bis 0,9\% innerhalb von 3 Jahren auf [415]. Diese setzen sich zusammen aus übersehenen Läsionen (miss rate), inkompletten Polypektomien sowie dem Auftreten schnell wachsender Tumoren [410, 411, 413, 498]. Als Grundlage für die Festlegung von Kontrolluntersuchungen nach Polypektomie kann eine Risikostratifizierung der Patienten nach dem Low-/ High-risk-Adenom-Konzept erfolgen, welches das Risiko für metachrone fortgeschrittene Läsionen anzeigt. Die Zuordnung von Patienten zu diesen Risikogruppen erfolgt nach Anzahl, Größe und Histologie der entfernten Adenome im Rahmen der Basisuntersuchung.

Nach dieser Einteilung definiert sich eine Low-risk-Adenom-Situation folgendermaßen: 1 - 2 Adenome, jeweils $<1 \mathrm{~cm}$, tubulär, nur LGIEN, Ausschluss von HGIEN und villöser Komponente.

Eine High-risk-Adenom-Situation (sog. advanced adenomas) definiert sich demgemäß wie folgt: $\geq 3$ tubuläre Adenome, $\geq 1$ Adenom mit $\geq 1 \mathrm{~cm}$, Adenom mit tubulo-villöser oder villöser Struktur, $\geq 1$ Adenom mit HGIEN, $\geq 10$ Adenome jeglicher Größe oder Histologie [499].

Auch in Piecemeal-Technik abgetragene flache oder sessile Adenome gehören in diese High-risk-Adenom-Gruppe.

Als generelles Konzept für die Terminierung von Kontrolluntersuchungen kann für die sog. Low-risk-Adenomgruppe ein Kontroll-Intervall von 5 Jahren, bei dann unauffälliger Untersuchung für erneut 5 Jahre angesetzt werden.

Für die sog. High-risk-Adenomgruppe sollte nach histologisch gesicherter kompletter Entfernung das Kontrollintervall 3 und bei dann unauffälligem Befund 5 Jahre betragen.

Nach Abtragung flacher oder sessiler Adenome in PiecemealTechnik ist insbesondere bei größeren Adenomen die Rezidivrate deutlich erhöht (9-28\%) [468, 500 - 503]. Der Einsatz von Argon Plasma Koagulation zur Entfernung von Restgewebe zur Sicherstellung einer kompletten Abtragung kann hilfreich sein [501, 503]. Allerdings fehlt dann die histologische Beurteilung. Die Sondergruppe von Patienten mit Z.n. Abtragung flacher oder sessiler Adenome in Piecemeal-Technik sollte wegen der erhöhten Lokalrezidivrate bzw. wegen der erhöhten Rate an metachronen Läsionen in kürzeren Abständen eine Kontroll-Koloskopie erhalten (2 - 6 Monate), dann nach 3 Jahren, dann nach 5 Jahren, im 
Individualfall ggf. früher. Im Falle größerer $(\geq 1 \mathrm{~cm})$ und insbesondere rechtsseitiger sessiler serratierter Adenome (früher als hyperplastische Polypen klassifiziert) scheint ein potenziell beschleunigtes Progressionsrisiko zum Karzinom (via serratierter Pathway) zu bestehen, sodass in diesen Fällen eine komplette Entfernung und ein kürzeres Kontrollintervall empfohlen wird (nach derzeitigem Wissensstand nach 3 Jahren).

Auch nach Abtragung von TSA, die vorwiegend im linken Kolon auftreten, ist aufgrund eines erhöhten Progressionsrisikos eine dem SSA analoge Nachsorge indiziert.

Bezüglich der Empfehlungen zur Nachsorge bei HNPCC, FAP und CED-Patienten (s. Leitlinienmanuskript von 2004, Abschnitt VIII.6.)

\subsection{Medikamentöse Sekundärprävention bei Adenomen} 6.23. Empfehlung (2008)

Eine medikamentöse Sekundärprophylaxe nach Polypektomie sollte derzeit außerhalb von Studien nicht erfolgen.

Empfehlungsgrad B, Level of Evidence 1b, starker Konsens

\section{Hintergrund}

Obwohl in mehreren prospektiv randomisierten Untersuchungen mit hoher Evidenzstärke (1b) ein geringer präventiver Effekt nach Einnahme von niedrigdosiertem ASS gefunden wurde [504, 505], kann aufgrund des geringen Effekts (Senkung der Rezidivadenomrate um max. 35\%) und der medikamentös bedingten Risiken [506] derzeit eine Einnahme zur Senkung des Rezidivrisikos von Adenomen nicht empfohlen werden. Gleiches gilt für die COX-2-Hemmer, für die eine Senkung der Adenomrezidivrate um 24 bis $45 \%$ erreicht wurde [79-81], die jedoch mit einer signifikant erhöhten Rate kardiovaskulärer Nebenwirkungen einhergingen [507, 508], die den potenziellen Nutzen aufwiegen [509]. Auch die Senkung der Adenomrezidivrate um 12\% durch Kalzium erscheint zu gering, um die längerfristige Einnahme für diese Indikation rechtfertigen zu können [510].

\section{Präoperative Diagnostik und Chirurgie}

$\nabla$

\subsection{Einleitung}

Im Folgenden werden allgemeine Gesichtspunkte der Diagnose und Therapie, soweit sie Kolon- und Rektumkarzinome gemeinsam betreffen, für beide Entitäten zusammenfassend aufgezeigt; spezielle diagnostische und therapeutische Aspekte werden gesondert aufgeführt.

Die Therapie kolorektaler Karzinome sollte grundsätzlich auf der Basis einer histologischen Untersuchung geplant werden. Als Karzinome gelten Veränderungen, bei denen atypische epitheliale Formationen in der Submukosa infiltrieren (pT1 oder mehr). Nicht einbezogen sind sog. Mukosakarzinome oder sog. intraepitheliale Karzinome (pTis), bei denen keine Metastasierung erfolgt und die durch lokale Abtragungen im Gesunden ausreichend behandelt werden.

\subsection{Definition von Kolon- und Rektumkarzinomen}

Die Grenze zwischen Kolon und Rektum wird unterschiedlich definiert. Die intraoperative Beurteilung anhand des Endes der Taeniae oder der peritonealen Umschlagsfalte ist individuell unterschiedlich und von Alter, Geschlecht und anderen Faktoren abhängig. Die präoperative Messung der Höhenangabe des Tumors mit dem flexiblen Endoskop ist unzuverlässig. Zuverlässiger sind die Höhenangaben mit dem starren Rektoskop. Die Anokutanlinie dient als distaler Messpunkt.

Nach dem internationalen Dokumentationssystem [511, 512] gelten als Rektumkarzinome Tumoren, deren aboraler Rand bei der Messung mit dem starren Rektoskop $16 \mathrm{~cm}$ oder weniger von der Anokutanlinie entfernt ist. Nach der UICC 2003 werden die Rektumkarzinome entsprechend ihrem Abstand von der Anokutanlinie in Karzinome des oberen Rektumdrittels (12$16 \mathrm{~cm})$, des mittleren Rektumdrittels $(6-<12 \mathrm{~cm})$ und des unteren Rektumdrittels $(<6 \mathrm{~cm})$ unterteilt [513].

Demgegenüber gelten in den USA $[514,515]$ als Kolonkarzinome Tumoren, die mehr als $12 \mathrm{~cm}$ und als Rektumkarzinome Tumoren, die $12 \mathrm{~cm}$ und weniger von der Linea anocutanea entfernt sind. Begründet wird dies mit der deutlich höheren Lokalrezidivrate bei Tumoren unterhalb von $12 \mathrm{~cm}$ [516].

\subsection{Definition Interdisziplinäre Tumorkonferenz}

\subsection{Konsensbasierte Empfehlung (2013)}

Alle Patienten mit KRK sollen nach Abschluss der Primärtherapie (z. B. Operation, Chemotherapie) in einer interdisziplinären Tumorkonferenz vorgestellt werden.

Bereits prätherapeutisch sollen Patienten in folgenden Konstellationen vorgestellt werden:

- jedes Rektumkarzinom

- jedes Kolonkarzinom im Stadium IV

- metachrone Fernmetastasen

- Lokalrezidive

- vor jeder lokal ablativen Maßnahme, z. B. RFA/LITT/SIRT

GCP, Konsens

\section{Hintergrund}

Um der Komplexität der Therapie des kolorektalen Karzinoms gerecht zu werden, ist eine Besprechung der Patienten in einer interdisziplinären Tumorkonferenz erforderlich. Mitglieder der Konferenz auf Facharztebene sollten u. a. sein: ein Gastroenterologe, ein Hämato-/Onkologe, ein Viszeralchirurg, ein Strahlentherapeut, ein Radiologe und ein Pathologe. Zur Beurteilung der primären oder sekundären Resektabilität von Lebermetastasen sollte ein erfahrener Leberchirurg hinzugezogen werden; ist dieser vor Ort nicht verfügbar, sollte eine externe konsiliarische Zweitmeinung eingeholt werden.

In bestimmten Fällen ist eine Vorstellung in der Tumorkonferenz vor Einleitung einer Therapie erforderlich. So ist z. B. bei Patienten mit Rektumkarzinom zu klären, ob eine neoadjuvante Therapie erfolgen sollte. Durch eine Vorstellung in der Tumorkonferenz und interdisziplinärer Festlegung des Therapiekonzepts konnte in einer Studie die Rate an befallenem zirkumferenziellen Rand im OP-Präparat deutlich gesenkt werden [517]. Liegen Fernmetastasen vor ist zu klären, ob ein rein palliatives Konzept einzuschlagen ist, oder ob durch eine primäre oder sekundäre Resektion von Metastasen (v. a. Lebermetastasen) eine Heilung möglich ist. Mit einer gehäuften Vorstellung von Patienten im Stadium UICC IV in der Tumorkonferenz nahm die Rate an Metastasenchirurgie zu [518].

Auch Patienten mit metachronen Fernmetastasen oder Lokalrezidiven im Verlauf sind zur Festlegung des weiteren Konzepts primär vorzustellen. Patienten bei denen ein lokalablatives Verfahren geplant ist, sollen ebenfalls vorgestellt werden, um alternative Therapiemöglichkeiten zu besprechen.

Im Falle von Kolonkarzinomen ohne Fernmetastasen erfolgt in der Regel primär eine onkologische Resektion des Karzinoms. Hier ist eine präoperative Vorstellung nicht erforderlich. 
In allen Fällen ist eine Vorstellung der Patienten nach Abschluss der Therapie erforderlich, z. B. nach Operation eines kolorektalen Karzinoms u. a. um dann die Indikation zur Durchführung einer adjuvanten Therapie zu besprechen. Hierdurch konnte in einer Studie aus Großbritannien die Rate an adjuvanter Chemotherapie und das Überleben der Patienten signifikant gesteigert werden [519].

Aber auch Patienten mit Fernmetastasen, bei denen primär eine Chemotherapie eingeleitet wurde, sollen im Verlauf erneut in der Tumorkonferenz (unter Hinzuziehung erfahrener Leber- bzw. Lungenchirurgen) vorgestellt werden, um eine mögliche sekundäre Resektabilität der Fernmetastasen zu klären.

\subsection{Präoperative Ausbreitungsdiagnostik (॰ Tab. 8)}

Tab. 8 Staging beim kolorektalen Karzinom

\begin{tabular}{|lll|}
\hline Untersuchung & KolonCa & RektumCa \\
\hline Komplette Koloskopie & $\times$ & $\times$ \\
\hline CEA & $\times$ & $\times$ \\
\hline Abdomensonografie & $\times$ & $\times$ \\
\hline Rö-Thorax & $\times$ & $\times$ \\
\hline Starre Rektoskopie & & $\times$ \\
\hline $\begin{array}{l}\text { MR(CT)-Becken mit Angabe Abstand des } \\
\text { Tumors zur mesorektalen Faszie }\end{array}$ & $\times$ \\
\hline $\begin{array}{l}\text { Rektale Endosonografie bei lokal } \\
\text { begrenztem Tumor }\end{array}$ & $\times$ \\
\hline
\end{tabular}

\subsubsection{Endoskopische Diagnostik}

\subsection{Empfehlung (2008)}

Folgende Untersuchungen sollten obligater Bestandteil der präoperativen Ausbreitungsdiagnostik beim kolorektalen Karzinom sein:

Empfehlungsgrad B, Level of Evidence 5, digital-rektale Untersuchung, Evidenzgrundlage [520 - 522]

Empfehlungsgrad A, Level of Evidence 4, komplette Koloskopie mit Biopsie, Evidenzgrundlage [520 - 522]

Empfehlungsgrad A, Level of Evidence 3b, im Falle einer nicht passierbaren Stenose Koloskopie 3-6 Monate postoperativ, Evidenzgrundlage [520 - 522],

Jeweils starker Konsens

\section{Hintergrund}

Die digital-rektale Untersuchung erlaubt eine orientierende Beurteilung der Sphinkterfunktion sowie der Tiefeninfiltration bei tiefsitzenden Rektumkarzinomen und lässt damit eine gewisse Abschätzung des Sphinktererhalts zu.

Vor der Therapie eines Patienten mit einem kolorektalen Karzinom muss eine Koloskopie mit Biopsie vorliegen. Da in bis zu 5\% der kolorektalen Karzinome synchrone Tumoren zu erwarten sind, die der intraoperativen Beurteilung entgehen könnten, ist eine Koloskopie des gesamten Kolons vorzunehmen [520 - 522]. Ist aus technischen Gründen eine komplette Koloskopie nicht möglich, kann ein alternatives radiologisches Verfahren eingesetzt werden. (s. Kapitel 6.1)

Stellenwert der Virtuellen Koloskopie (bei stenosierenden Tumoren und inkompletter Koloskopie)

\subsection{Empfehlung (2008)}

Bei inkompletter Koloskopie aufgrund eines stenosierenden Tumors kann präoperativ zusätzlich eine CT- oder MR-Kolonografie erfolgen. Postoperativ soll eine komplette Koloskopie erfolgen.

Empfehlungsgrad 0, Level of Evidence 4, [426], starker Konsens

\subsection{Empfehlung (2008)}

Bei inkompletter Koloskopie infolge anderer Ursachen (z. B. Adhäsionen) sollte eine CT- oder MR-Kolonografie erfolgen.

Empfehlungsgrad B, Level of Evidence 4, [426], starker Konsens

\section{Hintergrund}

Die virtuelle Kolonografie stellt hierfür ein vielversprechendes Verfahren [426]. Ist eine komplette Koloskopie aufgrund eines stenosierenden Prozesses nicht möglich, sollte eine Koloskopie ca. 3-6 Monate nach Resektion erfolgen. Ein präoperativer Kolonkontrasteinlauf ist von der Wertigkeit her eingeschränkt und bei Stenosen mit der Gefahr einer Ileusinduktion verbunden und wird daher nicht empfohlen.

\subsubsection{Bildgebende Verfahren}

7.4.2.1. Bedeutung einzelner bildgebender Verfahren (außer PET) zur Abklärung von Fernmetastasen bei der Primärbehandlung des kolorektalen Karzinoms

\subsection{Konsensbasierte Empfehlung (2013)}

Als Basisuntersuchungen des präoperativen Stagings des kolorektalen Karzinoms sollen die Ultraschalluntersuchung des Abdomens und die konventionelle Röntgenaufnahme des Thorax in 2 Ebenen erfolgen.

Im Falle eines unklaren Befunds oder des Verdachts auf Fernmetastasen oder Infiltration von Nachbarorganen oder umgebende Strukturen soll ein Mehrzeilen-CT des Abdomens und Beckens bzw. im Falle des Verdachts auf Lungenmetastasen ein CT des Thoraxes durchgeführt werden.

GCP, Konsens

\section{Hintergrund}

Ziel einer prätherapeutischen Bildgebung vor Therapieeinleitung ist zum einen, das Vorliegen von Fernmetastasen zu klären. So liegen zum Zeitpunkt der Erstdiagnose eines Kolonkarzinoms bei $25 \%$ der Patienten Fernmetastasen vor: in 13\% auf ein Organ beschränkt (M1a), in 12\% in mehr als einem Organ oder im Peritoneum (M1b). Lebermetastasen finden sich in 19\%, Lungenmetastasen in 3\%, Peritonealmetastasen in 9\%. Weitere Fernmetastasen in nichtregionären Lymphknoten $(2 \%)$, der Haut $(2 \%)$, des Ovars (1\%), der Knochen (<1\%) oder anderer Lokalisationen (2\%) sind selten.

Für das Rektumkarzinom beträgt die Häufigkeit von Fernmetastasen bei Erstdiagnose 18\%: in 12\% auf ein Organ beschränkt (M1a), in $6 \%$ in mehr als einem Organ oder im Peritoneum (M1b). Fernmetastasen in der Leber finden sich bei $15 \%$, Lungenmetastasen in $4 \%$. Weitere Fernmetastasen finden sich bei $3 \%$ im Peritoneum, bei $2 \%$ in nichtregionären Lymphknoten. Fernmetastasen in der Haut, Knochen, Gehirn, Ovar oder anderen Lokalisationen finden sich jeweils in weniger als $1 \%$ (Daten aus Klinischem Krebsregister der Chirurgischen Universitätsklinik Erlangen-Nürnberg).

Zur Klärung von Lebermetastasen soll primär die Abdomen-Sonografie eingesetzt werden (Sensitivität $63-86 \%$, Spezifität $98 \%$ [523 - 525]. Bei verdächtigen Befunden oder unzureichender Beurteilbarkeit der Leber in der Sonografie oder klinischen Verdacht auf das Vorliegen von Lebermetastasen ist ein MehrzeilenCT des Abdomens durchzuführen (Sensitivität 75-83\%, Spezifität 95 - $98 \%$ [523, 526]. Das Mehrzeilen-CT ist auch am besten geeignet für die Zuordnung der Metastasen zu den Lebervenen, den Hilusstrukturen sowie der Vena cava, die zur Beurteilung der Resektabilitiät von Lebermetastasen erforderlich ist (s. Kapitel 7.7.5). Das Ausmaß der Lebermetastasierung wird am besten durch die Magnetresonanztomografie erfasst (Sensitivität und 
Spezifität: MRT $80-88 \%$ und $93-97 \%$, CT $74-84 \%$ und $95-$ $96 \%)$. [523, 526].

Die kontrastmittelverstärkte Sonografie der Leber ist CT und MRT annähernd gleichwertig (Sensitivität 83-86\%, Spezifität 94 $98 \%$ ), setzt jedoch adäquate Qualitätsstandards (technische Ausrüstung und Erfahrung des Untersuchers) voraus [524, 525, 527]. Da das Mehrschicht-CT gleichzeitig eine Aussage zur lokalen Tumorausdehnung erlaubt (s. u.) besteht die Tendenz, statt bzw. in Ergänzung zu einem Sono-Abdomen primär ein Abdomen-CT durchzuführen. In Studien ergab sich bei Patienten mit Kolonkarzinomen allerdings durch einen routinemäßigen Einsatz eines präoperativen Abdomen-CTs lediglich in wenigen Fällen eine Änderung des weiteren Vorgehens $[528,529]$.

7.4.2.2. Stellenwert des präoperativen lokalen Stagings durch CT (MRT) beim Kolonkarzinom bez. lokaler Ausbreitung

\subsection{Konsensbasierte Empfehlung (2013)}

Mit einem präoperativen Staging-CT kann unterschieden werden zwischen Tumoren, die auf die Darmwand beschränkt sind und denen, die sie überschreiten. Die Identifikation des Nodalstatus gelingt jedoch signifikant schlechter. Die besten Ergebnisse bietet das Mehrzeilen-CT (MSCT).

GCP, Konsens

\section{Hintergrund}

Eine neoadjuvante Therapie beim nicht ausgedehnt metastasierten Kolonkarzinom wird derzeit nur in Ausnahmefällen (z.B. distales Sigmakarzinom mit sehr ausgedehnter regionärer lymphogener Metastasierung oder Tiefeninfiltration bis an die absehbaren Resektionsränder heranreichend) in Erwägung gezogen. Allerdings wird zunehmend diskutiert durch eine entsprechende präoperative Bildgebung den Therapiealgorithmus von Patienten zu optimieren, z. B. Selektion für eine laparoskopische Resektion oder Zuweisung von Patienten mit absehbarer multiviszeraler Resektion an erfahrene Zentren.

Die Aussagekraft der präoperativen Ultraschalluntersuchung des Abdomens ist diesbezüglich unzureichend. Erhebungen zur Genauigkeit des Kernspintomogramms zum lokalen Staging des Kolonkarzinoms liegen nicht vor. Das moderne Mehrschicht-CT (MSCT) erreicht eine hohe Sensitivität (86\%) und Spezifität (78\%) bez. der lokalen Tumorausdehnung, aber eine deutlich geringere Sensitivität (70\%) bei gleicher Spezifität (78\%) bez. der Detektion lokaler Lymphknotenmetastasen [530].

\subsubsection{Stellenwert der PET-CT}

7.4.2.3.1. bei der Primärdiagnostik des kolorektalen Karzinoms

\subsection{Statement (2013)}

Die PET/PET-CT hat keinen Stellenwert in der Ausbreitungsdiagnostik bei Erstdiagnose eines kolorektalen Karzinoms.

Level of Evidence 2b, de novo: [531 - 546], starker Konsens

\section{Hintergrund}

In der Literatursuche wurden 15 prospektive und 5 retrospektive Kohortenstudien und Fallserien identifiziert. In 13 Studien waren Patienten mit Kolon- und Rektumkarzinomen eingeschlossen, in 7 Studien nur Rektumkarzinompatienten. In 14 Studien wurden alle Patienten eingeschlossen, in 2 retrospektiven Studien nur Pat. mit lokal fortgeschrittenen Rektumkarzinomen, in einer Studie nur Patienten mit CEA-Erhöhung oder unklarer CT-Bildgebung. In den eingeschlossenen Studien wurde in 10 Fällen eine PET, in 6 Fällen eine PET-CT, in 4 Fällen eine PET-CT mit CT-Kolonografie durchgeführt. Verglichen wurde die PET mit CT \pm ande- ren Modalitäten in 14 Studien, mit einem MRT in 3 Studien; in 4 Studien wurde kein Vergleich vorgenommen. Referenz war in 18 Fällen die Histologie und der klinische Verlauf, in zwei Fällen das klinische Staging anhand der vorgenommenen Untersuchungen.

Die Sensitivität der PET für Fernmetastasen war hoch (75 - 100\%) und in einigen Fällen den Vergleichsmethoden überlegen [531, 536], in anderen Fällen nicht überlegen [532 - 534, 539]. Unklar ist die Qualität der Vergleichsmethoden. In neueren Studien mit Multidetector-Spiral-CT erscheint kein wesentlicher Unterschied nachweisbar [532, 533].

Die Sensitivität für Lymphknotenmetastasen war vorwiegend niedrig (29-85\%), bei 2 retrospektiven Rektumkarzinomstudien 44 und $85 \%$ und nicht besser als Vergleichsstudien (CT bzw. MRT) [531 - 539].

In den Studien führte der Einsatz der PET bzw. PET-CT in $2-27 \%$ zu einer Änderung des therapeutischen Vorgehens [532, 533, 536, 539 - 546].

Insgesamt zeigen die Daten keinen eindeutigen zusätzlichen Nutzen für die PET in der primären Diagnostik des kolorektalen Karzinoms.

\subsubsection{2. vor Resektion kolorektaler Lebermetastasen}

\subsection{Empfehlung (2013)}

Eine PET-CT kann bei Patienten mit resektablen Lebermetastasen eines kolorektalen Karzinoms mit dem Ziel der Vermeidung einer unnötigen Laparotomie durchgeführt werden.

Empfehlungsgrad 0, Level of Evidence 2b, de novo: [547, 548], Konsens

\subsection{Empfehlung (2013)}

Eine PET-CT soll nicht innerhalb von 4 Wochen nach Gabe einer systemischen Chemotherapie oder Antikörpertherapie durchgeführt werden, da die Sensitivität deutlich reduziert ist.

Empfehlungsgrad A, Level of Evidence 2b, de novo: [549-551], starker Konsens

\section{Hintergrund}

Zur Frage des Nutzens einer PET/PET-CT bei der Rezidivdiagnostik und dem Rezidivstaging bei Patienten mit kolorektalem Karzinom war im August 2011 vom IQWIG (Institut für Qualität und Wirtschaftlichkeit im Gesundheitswesen) ein Vorbericht vorgelegt worden [552]. Neben der Bewertung des Patienten-relevanten Nutzens sollte eine systematische Bewertung der prognostischen und diagnostischen Güte der PET/PET-CT durchgeführt werden. Die Literaturrecherche umfasste den Zeitraum bis August 2009. Aktuellere Publikationen wurden im Text erwähnt. Den hier getroffenen Leitlinienempfehlungen liegen die Evidenzbewertungen des IQWIG Berichts zugrunde. Der Empfehlungsgrad wurde unter zusätzlicher Einbeziehung der klinischen Bewertung des Verfahrens erarbeitet. Ferner wurden in einer neueren Literaturrecherche von August 2009 bis Dezember 2011 ein RCT in Abstraktform [548], zwei systematische Reviews [526, 553], eine prospektive Fallkontrollstudie [549] und eine retrospektive Fallserie [551] identifiziert, die in die Bewertung ebenfalls mit eingegangen sind (Einzelheiten siehe Evidenzbericht). Die bisherige Empfehlung mit einem Grad B für die PET/PET-CT-Untersuchung vor einer Resektion von kolorektalen Lebermetastasen bei einem FONG-Score > 2 der letzten Leitlinienaktualisierung 2008 (9.1.2.1) wurde geändert, da die Studie, die zu dieser Empfehlung führte, bis heute nicht als Vollmanuskript publiziert ist [554]. 
Zur Frage des Patienten-relevanten Nutzens der PET/PET-CT ist bislang eine RCT als Vollpublikation und 1 RCT in Abstraktform publiziert. Bei Patienten vor Resektion von Lebermetastasen eines KRK hat eine ergänzende PET/PET-CT keinen Einfluss auf das krankheitsfreie oder Gesamtüberleben des Patienten. Ob die ergänzende PET-CT-Untersuchung überflüssige Laparotomien als klinisch relevanten Endpunkt vermeiden kann, ist nicht vollständig geklärt. Die hier konsentierte Empfehlung stützt sich vor allem auf die voll publizierte Studie von Ruers [547], die jedoch methodische Schwächen aufweist. In dieser Studie wurden 150 Patienten mit kolorektalen Lebermetastasen, die zur Resektion vorgesehen waren, in zwei Arme randomisiert, CT oder CT plus 18F-FDG PET. Das primäre Studienziel wurde in der Publikation angegeben als die Rate an überflüssigen Laparotomien, die durch die PET-Untersuchung eingespart werden kann. Dieser Endpunkt ist für den Patienten relevant. Die Studie fand keinen signifikanten Unterschied im Überleben im PET-Arm, jedoch eine signifikante Verringerung der Zahl „überflüssiger Laparotomien“ im PET-Arm. So war im Kontrollarm die Rate an überflüssigen Laparotomien $45 \%$, im PET-Arm $28 \%$. Dies entsprach einer Risikoreduktion von $38 \%$ mit sehr großem Konfidenzintervall (95\% CI, $4-60 \%, p=0,042)$. Die Autoren schlussfolgern, dass man mit einer zusätzlichen PET vor Lebermetastasenresektion einem von 6 Patienten die Laparotomie ersparen könnte. Die sekundären Endpunkte DFS und OS waren wie folgt: DFS: 35,5\% versus $29,8 \%$ (p-Wert=0,194); OS: 61,3\% vs. 65,8\% (p-Wert=0,378). Die Studie wurde von der Konsensuskonferenz im Evidenzlevel herabgestuft (s. auch IQWIG-Bericht, Herabstufung von Ib auf II), da der bei der Studienplanung genannte primäre Studienendpunkt von dem in der Publikation angegeben abwich (ursprünglicher Endpunkt: Rate der Patienten, die nach 9 Monaten krankheitsfrei sind).

Eine weitere, bislang auf dem ASCO Jahresmeeting 2011 in Abstraktform vorgestellte multizentrische randomisierte Studie untersuchte ebenfalls diese Fragestellung [548]. Endpunkt dieser Studie war die Änderung im Patientenmanagement nach PETDiagnostik (nicht durchgeführte OP wegen zusätzlicher Befunde oder Ausweitung der OP im Vergleich zur Intention ohne/vor der PET-Diagnostik) in einem 2:1 randomisierten Design bei Patienten mit KRK, die für eine Leberresektion bei Lebermetastasen geeignet erschienen. Es wurden 404 Patienten randomisiert (270 Patienten im PET/CT-Arm, 134 Patienten ohne PET). Man fand keinen Unterschied hinsichtlich der Managementänderung zwischen beiden Armen. Der Endpunkt wurde somit nicht erreicht. Allerdings hatten - soweit dies aus der Präsentation zu erheben war - ca. 70\% der Patienten vor der PET Diagnostik eine Chemotherapie erhalten, was die Sensitivität der Untersuchungstechnik deutlich reduziert (s. u.). Kritisch anzumerken gilt ferner, dass der Endpunkt „Änderung im Patientenmanagement“ im Gegensatz zu „Verringerung überflüssiger Operationen“ nicht als Patienten-relevant gilt.

Eine Reihe von Untersuchungen weisen darauf hin, dass sich die Sensitivität einer PET deutlich reduziert, falls es innerhalb von 4 Wochen nach einer Chemotherapie durchgeführt wird (Evidenzlevel IIa-III). Daher wird eine PET in diesem Zeitraum nicht empfohlen, da zu viele falsch negative Fälle auftreten. Diese Fragestellung hat der IQWIG Bericht nicht bewertet. In einer größeren Fall-Kontroll-Studie, die die Sensitivität einer PET nach Chemotherapie nicht randomisiert prüfte, wurde 2010 publiziert [549]. Die Studie fand einen negativ prädiktiven Wert von nur $13,3 \%$ und einen positiven prädiktiven Wert von $94 \%$ mit einer Spezifität von $22,2 \%$ bei einer Accuracy von $85 \%$, falls die PET in- nerhalb von vier Wochen nach Chemotherapieende durchgeführt wurde. Die Autoren schlussfolgern, dass eine diagnostische PETUntersuchung kurz nach einer Chemotherapiegabe nicht sinnvoll sei. In einer retrospektiven Untersuchung aus Australien wurden PET-Ergebnisse von Patienten mit Lebermetastasen vor Leberresektion ausgewertet [555]. Auch diese Studie war klein und heterogen. 21 Patienten wurden präoperativ systemisch therapiert, 53 nicht. Korrekte Ergebnisse mittels PET wurden für 29\% nach Chemotherapie und $53 \%$ in der Nicht-Chemotherapie-Gruppe erzielt. Unterschätzte Befunde ergaben sich zu $52 \%$ in der Chemotherapie-Gruppe, nur zu $34 \%$ in der Nicht-ChemotherapieGruppe. Diese Studie gibt ebenfalls Hinweise darauf, dass eine PET-Untersuchung kurz nach einer Chemotherapie nicht sinnvoll ist. Eine weitere prospektive [550] und eine retrospektive Untersuchung [551] kamen zu ähnlichen Ergebnissen.

\subsubsection{Tumormarker}

7.10. Konsensbasierte Empfehlung (2013)

Der CEA-Wert sollte präoperativ bestimmt werden.

GCP, starker Konsens

\subsection{Empfehlung (2013)}

CA 19 - 9 erhöht die Aussagefähigkeit bezüglich des Vorliegens eines Rezidivs im Vergleich zu einer alleinigen CEA-Wert-Bestimmung nicht.

GCP, starker Konsens

\subsection{Empfehlung (2013)}

Die Bedeutung von CA 125 zur Diagnose von Ovarialmetastasen und als Verlaufsparameter zur weiteren Behandlung einer nachgewiesenen Peritonealkarzinose ist derzeit unklar.

GCP, starker Konsens

\section{Hintergrund}

Bei etwa 30\% aller kolorektaler Karzinome ist der Tumormarker CEA zum Zeitpunkt der Erstdiagnose erhöht. [Daten aus Klinischem Krebsregister der Chirurgischen Universitätsklinik Erlangen-Nürnberg]

Insbesondere in der Tumornachsorge ist dieser Tumormarker ein zuverlässiger Hinweis auf ein Rezidiv. Zudem ist es im Falle von Lebermetastasen ein unabhängiger Prognosefaktor.

Als weitere Tumormarker werden CA 19 - 9 und CA 125 diskutiert, wobei letzterer ein Marker der Peritonealkarzinose ist. [556 - 558]. Der Nachweis von zirkulierender DNA und sog. zirkulierender Tumorzellen im peripheren Blut wie im Knochenmark hat keinerlei Konsequenz.

\subsubsection{Spezielle Diagnostik beim Rektumkarzinom}

7.13. Konsensbasierte Empfehlung (2013)

Beim Rektumkarzinom sollte die starre Rektoskopie mit Höhenangabe des Tumorunterrandes obligater Bestandteil der präoperativen Diagnostik sein.

GCP, starker Konsens

\section{Hintergrund}

Die starre Rektoskopie ermöglicht eine genaue Bestimmung des Abstandes des distalen Tumorrandes von der Linea dentata und ist somit für die weitere Therapieentscheidung von wesentlicher Bedeutung. 


\subsection{Empfehlung/Statement (2013)}

Zum lokalen Staging eines Rektumkarzinoms sollte vorzugsweise eine MRT, im Falle eines mutmaßlichen T1-Karzinoms eine Endosonografie, durchgeführt werden.

Empfehlungsgrad B, Level of Evidence 2b, de novo: [559-567], starker Konsens

\subsection{Statement (2013)}

Für T1-Karzinome ist die CT nicht geeignet.

Level of Evidence 3, de novo: [559 - 567], starker Konsens

\subsection{Statement (2013)}

Die Wertigkeit aller bildgebenden Verfahren zur Beurteilung des Lymphknotenstatus ist mit erheblicher diagnostischer Unsicherheit behaftet.

Level of Evidence 2b, de novo: [561, 562], starker Konsens

\subsection{Empfehlung (2013)}

Die Befundbeschreibung soll eine Aussage über den Abstand zur mesorektalen Faszie beinhalten.

GCP, starker Konsens

\section{Hintergrund}

Beim Rektumkarzinom kommt dem lokalen Staging eine entscheidende Bedeutung bei der weiteren Therapieplanung zu. Während bei einem Low-risk-T1-Karzinom eine lokale Abtragung ausreichend ist, ist bei High-risk-T1- sowie -T2-Karzinomen eine Resektion nach onkologischen Kriterien erforderlich. Bei Nachweis einer Tumorinfiltration in das Mesorektum (T3) wird in Deutschland eine neoadjuvante Therapie, bei Nachweis einer Infiltration in Nachbarorgane (T4) in Form einer neoadjuvanten Radiochemotherapie empfohlen. Bei T3-Karzinomen liegen Daten vor die zeigen, dass dem Ausmaß der Beteiligung des Mesorektums insbesondere der Abstand von der mesorektalen Faszie eine wichtige prognostische Bedeutung zukommt [559]. Diese Ebene stellt bei der TME die zirkumferenzielle Resektionsgrenze (CRM) dar. Ist die mesorektale Faszie infiltriert bzw. reicht der Tumor bis $1 \mathrm{~mm}$ an die Faszie heran $\left(\mathrm{CRM}^{+}\right)$ist das Lokalrezidivrisiko deutlich erhöht [560]. Ein weiterer Prognosefaktor sind befallene Lymphknoten [559].

Bei der Literatursuche zur Wertigkeit verschiedener Verfahren im lokalen Staging von Rektumkarzinomen musste eine Reihe von Studien ausgeschlossen werden, da das Studienkollektiv auch Patienten nach erfolgter Radio- bzw. Radiochemotherapie enthielt. Für weitere Einzelheiten sei auf den Evidenzbericht verwiesen.

Die Genauigkeit der einzelnen diagnostischen Verfahren hängt von den technischen Voraussetzungen der Geräte (z. B. Multidetector-Spiral-CT vs. 1-Schicht CT) und der lokalen Expertise ab. Bei höhergradigen Stenosen oder Tumoren im proximalen Rektum ist eine Endosonografie häufig technisch nicht durchführbar. In einer Metaanalyse, in der die Daten zur Endosonografie, zum MRT und zum CT bis 2002 analysiert wurden, wies die Endosonografie die höchste Genauigkeit bei T1-Karzinomen auf [561]. Die hohe Sensitivität und Spezifität der Endosonografie konnte in einer neueren Metaanalyse bestätigt werden [562]. Das MRT mit endorektaler Spule stellt eine mögliche Alternative zum EUS dar, ist aber mit höheren Kosten verbunden und wird von den Patienten als unangenehm empfunden und ist an sehr wenigen Standorten etabliert. Das CT ist für die Festlegung von T1-Karzinomen nicht geeignet.

Bei der Differenzierung von T2 und T3-Karzinomen wies die Endosonografie wiederum eine höhere Sensitivität im Vergleich zu
MRT und CT auf bei vergleichbarer Spezifität [561]. Bei T4-Karzinomen gab es in der Metaanalyse keine wesentlichen Unterschiede zwischen den Untersuchungsmodalitäten. In einzelnen neueren Kohorten wurden für das MRT und vereinzelt das Spiral-CT für Tumoren mit Infiltration des Mesorektums (> T2) höhere Sensitivitäten gezeigt [563 - 567], wobei im unteren Drittel die Genauigkeit für das CT deutlich geringer war als in den oberen zwei Dritteln [568]. Für Fragestellungen, die eine Darstellung der mesorektalen Faszie und die Beziehung des Tumors zu ihr erfordern, weist das MRT aktuell die höchste Sensitivität auf [569]. Das Spiral-CT stellt eine mögliche Alternative dar [563], in der Endosonografie lässt sich die Faszie nicht darstellen.

Bei der Beurteilung der Lymphknoten sind Sensitivität (55 - 73\%) und Spezifität ( $74-78 \%$ ) aller Verfahren derzeit unbefriedigend [561, 562]. Dies ist u. a. dadurch bedingt, dass einerseits eine reaktive Lymphknotenvergrößerung auftritt, andererseits auch Lymphknoten von $5 \mathrm{~mm}$ und kleiner Metastasen enthalten können. Aus diesem Grund sollte die Indikation zu einer neoadjuvanten Therapie sehr zurückhaltend gestellt werden, wenn sie allein auf der Beschreibung suspekter Lymphknoten in der prätherapeutischen Bildgebung basiert.

Ferner ist zu bedenken, dass die Genauigkeit der einzelnen Verfahren wesentlich von der lokalen Expertise abhängt, was insbesondere auch für die Kernspintomografie gilt.

Unter Berücksichtigung insbesondere der Möglichkeit der Darstellung der mesorektalen Faszie wird von vielen Experten aktuell das MRT - mit Ausnahme von frühen Karzinomen - für das lokale Staging des Rektumkarzinoms bevorzugt.

Mit Ausnahme der Kurzzeitbestrahlung wird durch eine neoadjuvante Therapie die Genauigkeit der einzelnen diagnostischen Verfahren eingeschränkt (s. Evidenzbericht).

\subsection{Empfehlung/GCP (2008)}

Folgende Untersuchungen können im Einzelfall nützlich sein:

- Sphinktermanometrie

Empfehlungsgrad 0, Level of Evidence 4

- Gynäkologische Untersuchung

Empfehlungsgrad GCP

- Zystoskopie

Empfehlungsgrad GCP, Jeweils starker Konsens

\section{Hintergrund}

Die Sphinktermanometrie hat im Allgemeinen keinen Einfluss auf den Therapieentscheid bezüglich des Sphinktererhalts über das Ergebnis der rektal-digitalen Untersuchung und der differenzierten Anamnese hinaus. In unklaren Fällen kann sie eine Entscheidung bezüglich des Sphinktererhalts erleichtern.

Bei Verdacht auf eine Infiltration der Blase kann eine Zystoskopie hilfreich sein, bei Verdacht auf Infiltration von Vagina, Uterus oder Adenexe sollte eine gynäkologische Untersuchung erfolgen. Entgegen der früheren Leitlinie wird ein Urinsediment bei Rektum- oder Sigmakarzinomen nicht mehr empfohlen, da die Untersuchung zu unspezifisch ist.

\subsection{Chirurgische Therapie mit kurativem Ziel}

7.5.1. Intraoperatives Staging

\subsection{Konsensbasierte Empfehlung (2013)}

Eine intraoperative Inspektion und, bei offener Operation, die Palpation der Leber sollte in jedem Fall, d.h. auch bei unauffälligem präoperativen Staging, erfolgen. 
Bei suffizienter präoperativer Diagnostik rechtfertigt der diagnostische Zugewinn nicht den Aufwand einer intraoperativen Sonografie zur Suche nach weiteren Metastasen.

GCP, Konsens

\section{Hintergrund}

Aufgrund der zwischenzeitlichen Qualität von Kernspintomografie und Computer-Tomografie werden bei einer Laparotomie in der Regel nur zusätzliche subseröse Lebermetastasen $(<2 \mathrm{~mm})$ durch die intraoperative Inspektion und Palpation entdeckt.

Allerdings liegen die Sensitivität und der positiv prädiktive Wert der intraoperativen Ultraschalluntersuchung mit Kontrastmittel sehr hoch (in einer einzigen Serie mit 24 Patienten bei je 100\%) [570].

\subsubsection{Intraoperative pathologische Diagnostik}

Allgemein ist die Indikation zur Schnellschnittuntersuchung nur bei sich unmittelbar ergebenden Konsequenzen zu stellen. Häufigste Indikation ist die Verifikation von Strukturen mit Verdacht auf Fernmetastasen, z. B. am Peritoneum, in der Leber oder in nicht regionären (z. B. paraaortalen) Lymphknoten.

Bei chirurgischen lokalen Exzisionen (Vollwandexzisionen) stellt sich die sehr wichtige Frage, ob ein zuvor bioptisch gesichertes Karzinom seitlich oder basal im Gesunden entfernt wurde.

Allerdings kann diese nicht mit hinreichender Sicherheit intraoperativ durch Schnellschnittuntersuchung geklärt werden.

Bei einem tiefsitzenden Rektumkarzinom kann nach zunächst vorgenommener tiefer anteriorer Resektion gelegentlich die Schnellschnittuntersuchung des aboralen Resektionsrandes für die Indikation zu einer Erweiterung zur Rektumexstirpation von Bedeutung sein.

Bei möglicher Segment- und tubulärer Resektion wegen großer, polypöser, insbesondere villöser Tumoren des Kolons, bei denen prätherapeutisch eine Karzinomdiagnose nicht gesichert werden konnte, ist eine Dignitätsbeurteilung im Schnellschnitt aus untersuchungstechnischen Gründen (Untersuchung multipler Gewebsblöcke!) häufig nicht möglich. Daher empfiehlt sich in diesen Situationen in der Regel die radikale Tumoroperation.

Bei Adhärenz eines Tumors an Nachbarorganen ist makroskopisch nicht sicher zu klären, ob es sich um eine Infiltration des Karzinoms in das Nachbarorgan oder nur um eine peritumoröse Entzündungsreaktion handelt. In solchen Fällen sollten Biopsien und Schnellschnittuntersuchungen strikt vermieden werden, da hierbei stets die Gefahr einer örtlichen Tumorzelldissemination besteht, was mit einer signifikanten Verringerung der Überlebenschancen einhergeht [571]. Dies begründet die En bloc-Resektion in allen Fällen von Tumoradhärenzen zu benachbarten Organen oder sonstigen Strukturen (s. Abschnitt 7.7.2, multiviszerale Resektion).

\subsection{Konsensbasierte Empfehlung (2013)}

Sofern durch die Bildgebung keine eindeutige diagnostische Zuordnung von unklaren Leberläsionen getroffen werden kann, sollte eine histologische Sicherung erfolgen.

GCP, Konsens

\section{Hintergrund}

Bei unklaren Läsionen der Leber (s. u.) sollte, sofern sich hieraus unmittelbarer Handlungsbedarf ergibt, eine histologische Sicherung möglichst durch eine Nadelbiopsie durch gesundes Leberparenchym hindurch erfolgen. Inzisionsbiopsien sind strikt zu vermeiden. Bei kleineren Läsionen kann auch eine vollständige Exzision im Sinne einer Exzisionsbiopsie erfolgen.

\subsection{Konsensbasiertes Statement (2013)}

Die Sentinel-Node-Biopsie (Wächterlymphknoten-Exzision) hat keinen Stellenwert beim kolorektalen Karzinom.

GCP, Konsens

\section{Hintergrund}

Bei malignen Melanomen und Mammakarzinomen wird die Sentinel-Node-Biopsie durchgeführt, um bei Patienten mit histologisch negativen Pförtnerlymphknoten eine weitergehende Dissektion mit Erhöhung der Morbititätsrate zu vermeiden.

Mit Einführung der laparoskopischen Operationstechniken wurde diskutiert, ob auch bei kolorektalen Karzinomen eingeschränkte Resektionsverfahren durchgeführt werden können, wenn Sentinel-Node-Biopsien negative Pförtnerlymphknoten erbringen.

Außerdem wurde die Frage gestellt, ob sich bei Ultrastaging (Immunhistochemische Aufarbeitung) des Pförtnerlymphknotens Änderungen im Tumorstadium ergeben könnten, mit entsprechender Notwendigkeit für eine adjuvante Therapie [572, 573].

\subsubsection{Radikalchirurgische Therapie des Kolonkarzinoms}

7.5.2.1. Ausmaß der Lymphknotendissektion

Im Falle einer lymphogenen Metastasierung des Kolonkarzinoms erfolgt diese nach regelhaftem Metastasierungsmuster, nämlich zunächst longitudinal, zu beiden Seiten des Tumors in die parakolischen Lymphknoten, im weiteren zu den intermediären Lymphknoten entlang der radiären Arterien bis hin zu den zentralen Lymphknoten am Stamm der versorgenden Arterien. Hierbei erfolgt die parakolische Metastasierung nie über eine längere Distanz von mehr als $10 \mathrm{~cm}$ [574-576].

Aus der Durchtrennung der zentralen Arterien ergibt sich das Ausmaß der Darmresektion, wobei im Falle rechtsseitiger Karzinome Lymphknotenmetastasen am terminalen Ileum nur sehr selten und dann nur bei sehr weit fortgeschrittenen Karzinomen vorkommen [577]. Deshalb ist eine Resektion des terminalen Ileums von max. $10 \mathrm{~cm}$ bei der Hemikolektomie rechts ausreichend.

\section{Onkologische Grundsätze}

Bei der Kolonkarzinomchirurgie korreliert die Lymphknotenausbeute auch im Falle nodal negativer Karzinome (UICC II) mit der Prognose [578, 579].

Vorgehen bei Karzinomen des Zökums und des Colon ascendens Karzinome in diesem Bereich metastasieren nach zentral über die Arteria ileocolica und die Arteria colica dextra. Entsprechend müssen beide Gefäße zentral durchtrennt werden. Allerdings liegt eine echte Arteria colica dextra mit Abgang aus der Arteria mesenterica superior nur in weniger als $15 \%$ aller Fälle vor [580]. Demnach werden bei nicht angelegtem Gefäß nach rechts ziehende Äste aus dem Hauptstamm der Arteria colica media zentral durchtrennt. Anteile des Omentum majus müssen nur bei direktem Tumorkontakt mitreseziert werden.

Vorgehen bei Karzinomen der rechten Flexur und des rechten Colon transversum

Bei der erweiterten Hemikolektomie rechts werden die Arteria ileocolica, die Arteria colica dextra (soweit vorhanden) und die Arteria colica media zentral ligiert. Entsprechend ergibt sich 
eine distale Resektionsgrenze im Bereich des linken Colon transversum. Da in dieser Tumorlokalisation auch eine lymphogene Metastasierung über das große Netz Richtung Magenantrum und weiter zum Pankreaskopf hin [575] stattfindet, werden neben der Skelletierung der großen Magenkurvatur und Resektion der Gastroepiploica-dextra-Arkade und damit der rechtsseitigen Omentumanteile auch die Lymphknoten über dem Pankreaskopf disseziert.

Vorgehen bei Karzinomen des mittleren Colon transversum

Diese Tumoren metastasieren einerseits über die Arteria colica media nach zentral Richtung A. mesenterica superior, andererseits über die Arteria colica sinistra Richtung Arteria mesenterica inferior. Eine Transversumresektion schließt beide Kolonflexuren mit ein, bei Metastasierung über das Omentum majus Richtung große Magenkurvatur muss korrespondierend zur Tumorlokalisation auch eine Omentumresektion unter Beachtung des Arkadenprinzips (Einbeziehung der Omentumarterie innerhalb einer Arkade zu beiden Seiten des Karzinoms von $10 \mathrm{~cm}$ ) sowie eine Skelettierung der großen Magenkurvatur mit Entfernung dieser Lymphknoten durchgeführt werden.

Vorgehen bei Karzinomen des distalen Colon transversum und der linken Kolonflexur

Die Metastasierung verläuft hier nach rechts über die Arteria colica media, nach links über die Arteria colica sinistra. Somit wird die Arteria colica media zentral, die Arteria colica sinistra am Abgang aus der Arteria mesenterica inferior abgesetzt. Ein Vorteil einer höheren Radikalität durch zentrale Absetzung der Arteria mesenterica inferior ist nicht belegt. Eine Kontinuitätswiederherstellung kann als Ascendo-Sigmoideostomie erfolgen. Wegen der Metastasierung über das große Netz Richtung große Magenkurvatur müssen entsprechend die linksseitigen Omentumanteile unter Dissektion der Arkade an der großen Magenkurvatur mit entfernt werden. Bei fortgeschrittenen Tumoren in diesem Bereich können auch Lymphknoten am linken Pankreasunterrand befallen sein, sodass auch diese vom Isthmus bis zum Pankreasschwanz disseziert werden.

Vorgehen bei Karzinomen des Colon descendens

Bei einer Metastasierung über die Arteria colica sinistra und den Arteriae sigmoideae nach zentral ist hier eine Hemikolektomie links mit zentraler Durchtrennung der Arteria mesenterica inferior erforderlich. Die distale Resektionsgrenze liegt dabei im oberen Rektumdrittel, die proximale im Flexurbereich links. Entsprechend müssen eventuell adhärente Omentumanteile mit reseziert werden.

\section{Vorgehen bei Karzinomen des Sigmas}

Diese Tumoren metastasieren über die Arteriae sigmoideae zum Stamm der Arteria mesenterica inferior. Die proximale Kolondurchtrennung liegt dabei im Colon descendens mit zentraler Durchtrennung der Arteria mesenterica inferior; bei der distalen Darmdurchtrennung greifen auch die Richtlinien für das Rektumkarzinom im oberen Rektumdrittel, wobei hier mindestens ein Sicherheitsabstand nach distal von $5 \mathrm{~cm}$ zum Tumorunterrand eingehalten werden muss mit Durchtrennung des Mesorektums ohne „Coning“ (Ausdünnung proximalwärts).

Hintergrund: Bei 2-4\% der Patienten liegen Lymphknotenmetastasen abgangsnah am Stamm der Arteria mesenterica inferior vor $[581,582]$.

\subsubsection{Komplette mesokolische Exzision (CME)}

\subsection{Konsensbasierte Empfehlung (2013)}

Die chirurgische Therapie des Kolonkarzinoms sollte die komplette mesokolische Exzision beinhalten.

GCP, Konsens

\section{Hintergrund}

Analog zum Mesorektum existiert in gleicher Weise ein Mesokolon, welches als beidseitige Hülle die Lymphknoten an den versorgenden Arterien umfasst.

Analog zur TME beim Rektumkarzinom wird die CME bei der Operation von Kolonkarzinomen angewandt, um bei der Präparation in vorgegebenen anatomischen Schichten mit zentraler Absetzung der versorgenden Gefäße eine maximale lokale Radikalität mit maximaler Lymphknotenausbeute zu erzielen, und zudem auf die Erhaltung der beiden mesokolischen Schichten („Faszien“) geachtet.

Die komplette mesokolische Exzision beim Kolonkarzinom ist geeignet, eine maximale lokale Radikalität mit hoher Lymphknotenausbeute zu erreichen.

Sie führt zu qualitativ höherwertigen Präparaten [583, 584]. Höhere Komplikationsraten scheinen mit Erhöhung der Radikalität nicht verbunden zu sein [585]. Die bisherigen Daten deuten auf eine Verbesserung der Überlebensraten bei konsequenter Durchführung der CME hin [583].

Die morphometrische Untersuchung des Kolonpräparats kann zukünftig zur objektiven Bewertung von Kolonkarzinompräparaten herangezogen werden.

\subsubsection{Lokalablative Verfahren bei Lebermetastasen \\ 7.5.2.3.1. Radiofrequenzablation (RFA)}

7.23. Empfehlung (2013)

Eine RFA kann durchgeführt werden, wenn nicht resektable Lebermetastasen vorliegen oder der Allgemeinzustand des Patienten eine Resektion nicht zulässt, insbesondere nach vorangegangener Leberresektion.

Empfehlungsgrad 0, Level of Evidence 3a, de novo: [586-588], starker Konsens

\section{Hintergrund}

Die derzeitige Evidenzlage zur Sicherheit und Effektivität der Radiofrequenzablation bei kolorektalen Lebermetastasen ist angemessen, um die Verwendung dieser Methode bei Patienten zu befürworten bei denen entweder nicht resektable Lebermetastasen vorliegen, deren Zustand eine Resektion nicht zulässt, oder bei denen zuvor eine Leberresektion durchgeführt worden ist [586]. Die RFA kann auch primär in Kombination mit der chirurgischen Resektion durchgeführt werden.

Neuere Arbeiten legen die Vermutung nahe, dass bei solitären Lebermetastasen $<3 \mathrm{~cm}$ mittels RFA ähnlich gute Ergebnisse wie durch die Resektion erzielt werden können [587, 588]. Die bisherige Datenlage hierzu ist aber widersprüchlich und es fehlen weiterhin vergleichende kontrolliert-randomisierte Studien.

\subsection{Selective Internal Radiation Therapy (SIRT)}

\subsection{Empfehlung (2013)}

Eine SIRT zur Behandlung von disseminierten Lebermetastasen bei KRK sollte nur bei Patienten, für die keine andere Therapieoption infrage kommt, und dann nur innerhalb klinischer Studien durchgeführt werden.

Empfehlungsgrad B, Level of Evidence 2a, de novo: [589, 590], Konsens 


\section{Hintergrund}

Patienten mit fehlender oder allenfalls begrenzter extrahepatischer Metastasierung, die zudem systemtherapeutisch austherapiert sind, zeigen in einzelnen Studien mit SIRT (gleichbedeutend mit dem Begriff Radioembolisation) ein verlängertes medianes Überleben sowie ein längeres Intervall bis zum Progress von Lebermetastasen. Für eine endgültige Bewertung, insbesondere auf Überleben und Lebensqualität liegen zu wenige Daten vor. Deshalb sollten Patienten, die für eine SIRT infrage kämen, nur innerhalb klinischer Studien behandelt werden [589, 590].

\subsection{Laserinduzierte interstitielle Thermotherapie (LITT)}

\subsection{Empfehlung (2013)}

Eine LITT zur Behandlung von Lebermetastasen bei KRK sollte nur innerhalb klinischer Studien durchgeführt werden.

Empfehlungsgrad B, Level of Evidence 4, de novo: [591, 592], starker Konsens

\section{Hintergrund}

In einzelnen Fallserien war die interstitielle Laserthermoablation eine sichere und wirksame Behandlung für Patienten mit inoperablen Lebermetastasen kolorektaler Karzinome [591, 592]. Daten zum Effizienzvergleich mit der perkutanen Radiofrequenzablation liegen nicht vor. Für eine endgültige Bewertung, insbesondere auf Überleben und Lebensqualität liegen zu wenige Daten vor. Deshalb sollten Patienten, die für eine LITT infrage kämen, nur innerhalb klinischer Studien behandelt werden.

\subsubsection{Radikalchirurgische Therapie des Rektumkarzinoms} Die kurative Therapie des Rektumkarzinoms erfordert in der Regel neben der Resektion des Primärtumors im Gesunden die partielle oder totale Entfernung des Mesorektums und damit des regionären Lymphabflussgebiets (sog. radikale Resektion nach internationalem Dokumentationssystem für das kolorektale Karzinom [511, 512]. Nur in streng selektionierten Fällen ist eine kurative Resektion durch lokale Maßnahmen möglich. Folgende Operationsverfahren sind bei Einhaltung der Kriterien der onkologischen Chirurgie als gleichwertig anzusehen, wobei die Indikationsstellung von der Tumorlokalisation, insbesondere der Beziehung zur Linea dentata und dem Levatorschenkel, der Tiefeninfiltration und der Sphinkterfunktion abhängig ist:

- die (tiefe) anteriore Rektumresektion,

- die abdomino-perineale Rektumexstirpation,

- die intersphinktere Rektumresektion (auch als abdominoperanale Rektumresektion bezeichnet). Diese Operation setzt besondere Erfahrungen voraus.

$\mathrm{Zu}$ beachten ist, dass auch bei der tiefen anterioren Rektumresektion sehr häufig zur Erreichung eines ausreichenden Sicherheitsabstandes nach aboral eine intersphinktäre Präparation erforderlich ist. Hiermit ist jedoch nicht das Operationsverfahren im Sinne der abdominoperanalen Präparation zu verwechseln.

Nach Möglichkeit sind kontinenzerhaltende Verfahren unter Abwägung der zu erwartenden späteren Lebensqualität zu bevorzugen. Bei schlechter Sphinkterfunktion sollte an Stelle einer tiefen Resektion die Anlage einer permanenten Kolostomie bevorzugt werden, die je nach Sicherheitsabstand vom Beckenboden als Rektumexstirpation oder Beckenboden-erhaltend ausgeführt wird.

\subsubsection{Allgemeine onkologische Grundsätze}

Die operative Therapie sollte folgende Grundsätze beinhalten:

Die Entfernung des regionären Lymphabflussgebiets mit Absetzung der A. mesenterica inferior zumindest distal des Abgangs der A. colica sinistra. Die abgangsnahe Unterbindung der A. mesenterica inferior hat keine prognostische Bedeutung, sie wird aber häufig aus operationstechnischen Gründen zur ausreichenden Mobilisation des linken Hemikolons zur Rekonstruktion durchgeführt [593]. Allerdings zeigen anatomische Studien, dass in vielen Fällen auch ohne zentrale Absetzung eine tiefe Anastomose möglich ist [594]. Der Wert einer Dissektion der Lymphknoten am Stamm der A. mesenterica inferior proximal des Abgangs der A. colica sinistra ist nicht gesichert (Evidenzstärke 2b) [595 - 598].

- Die komplette Entfernung des Mesorektums beim Karzinom des mittleren und unteren Rektumdrittels und die partielle Mesorektumexzision beim Karzinom des oberen Rektumdrittels durch scharfe Dissektion entlang anatomischer Strukturen zwischen Fascia pelvis visceralis und parietalis (totale mesorektale Exzision - TME) [599, 600].

- Die Einhaltung eines angemessenen Sicherheitsabstands (s. u.).

- In der Regel die En-Bloc-Resektion von tumoradhärenten Organen (multiviszerale Resektion) zur Vermeidung einer örtlichen Tumorzelldissemination [601].

- Die Schonung der autonomen Beckennerven (Nn. hypogastrici, Plexus hypogastrici inferiores et superior) [602, 603].

7.26. Empfehlung (2013)

Die systematische Dissektion der lateralen Lymphknoten entlang der Art iliaca interna und ihrer Äste soll ohne begründeten Metastasenverdacht nicht durchgeführt werden. Sie erhöht die perioperative Morbidität, ohne dass onkologische Vorteile belegt sind. Empfehlungsgrad A, Level of Evidence 1b, de novo: [604-607], starker Konsens

\subsubsection{Vorgehen bei Tumoren des oberen Rektumdrittels \\ 7.27. Empfehlung (2013)}

Bei Tumoren des oberen Rektumdrittels erfolgt die Durchtrennung des Rektums mit partieller Mesorektumexzision $5 \mathrm{~cm}$ distal des makroskopischen Tumorrands, gemessen in vivo. Das Mesorektum sollte horizontal ohne proximalwärtige Ausdünnung durchtrennt werden (kein Coning).

Empfehlungsgrad B, Level of Evidence 3b, de novo: [599, 608 612], starker Konsens

\section{Hintergrund}

Die Begründung dieses Vorgehens [611,612] liegt darin, dass bei T3- und T4-Tumoren in seltenen Fällen Satellitenknoten oder Lymphknotenmetastasen in bis zu $4 \mathrm{~cm}$ distal des makroskopischen Tumorrands, gemessen am histologischen Schnitt nach Fixation des nicht ausgespannten Präparats, vorkommen können.

\subsubsection{Vorgehen bei Tumoren des mittleren und unteren} Rektumdrittels

\subsection{Empfehlung (2008)}

Bei Tumoren des mittleren und unteren Rektumdrittels erfolgt die totale Mesorektumexzision (TME) bis zum Beckenboden unter Schonung des Plexus hypogastricus superior, der Nn. hypogastrici und der Plexus hypogastrici inferiores.

Empfehlungsgrad A, Level of Evidence 1b, [602, 613, 614], starker Konsens 


\subsection{Empfehlung (2008)}

Bei Low-Grade-Tumoren guter oder mäßiger Differenzierung des unteren Rektumdrittels ist ein Sicherheitsabstand von $1-2 \mathrm{~cm}$ in situ ausreichend. Bei High-Grade-Tumoren (G3/4) ist ein größerer Sicherheitsabstand anzustreben.

Empfehlungsgrad B, Level of Evidence 2b, [600, 615 -618], starker Konsens

\subsection{Empfehlung (2013)}

Nach neoadjuvanter Radiochemotherapie kann zur Abwendung einer ansonsten notwendigen Exstirpation auch ein aboraler Abstand von $0,5 \mathrm{~cm}$ akzeptiert werden. Die Tumorfreiheit des aboralen Resektionsrandes sollte durch intraoperativen Schnellschnitt gesichert werden.

Empfehlungsgrad B, Level of Evidence 2b, de novo: [619, 620], Konsens

\section{Hintergrund}

Bei Karzinomen des unteren Drittels kann als Alternative zu der ansonsten erforderlichen Rektumexstirpation die intersphinktere Rektumresektion (auch als abdomino-peranale Rektumresektion bezeichnet) durchgeführt werden, wenn - unter Wahrung der o.g. Sicherheitsabstände - die puborektale Schlinge nicht infiltriert ist. Diese Operation setzt besondere Erfahrung voraus.

\subsubsection{Rekonstruktion nach total mesorektaler Exzision}

Nach totaler mesorektaler Resektion mit nachfolgender spinkternaher Anastomose ist potenziell mit u. U. erheblichen funktionellen Störungen zu rechnen. Diese sind abhängig von der Wahl des Rekonstruktionsverfahrens. Als Möglichkeiten stehen zur Verfügung:

- gerade koloanale Anastomose

- der Kolon-J-Pouch

- die transverse Coloplastik

- die Seit-zu-End-Anastomose

\subsection{Empfehlung (2013)}

Bei der Rekonstruktion nach tiefer anteriorer Resektion soll wegen der besseren funktionellen Ergebnisse in der Regel (wenn anatomisch machbar) keine gerade colo-anale Anastomose angelegt werden.

Empfehlungsgrad A, Level of Evidence 1a, de novo: [621], starker Konsens

\subsection{Statement (2013)}

Von den verschiedenen Rekonstruktions-Formen sind die Vorteile im funktionellen Ergebnis des Kolon-J-Pouches am besten belegt. Level of Evidence 1a, de novo: [621, 622], mehrheitliche Zustimmung

\subsection{Statement (2013)}

Unter funktionellen Gesichtspunkten ist die transverse Koloplastie dem Kolon-J-Pouch unterlegen.

Level of Evidence 1b, de novo: [622, 623], mehrheitliche Zustimmung

\subsection{Statement (2013)}

Möglicherweise ist die Seit-zu-End-Anastomose dem Kolon-J-Pouch ebenbürtig.

Level of Evidence 2a, de novo: [624], starker Konsens

\section{Hintergrund}

Die Nachteile einer geraden colo-analen Anastomose sind hinreichend belegt und wirken sich vor allem während der ersten beiden postoperativen Jahre in erhöhter Stuhlfrequenz sowie schlechterer Kontinenz und Lebensqualität aus [621]. Bei Anlage eines J-Pouches sollte zur Vermeidung von Entleerungsproblemen die Schenkellänge nicht über $6 \mathrm{~cm}$ liegen [625]. Die einzige größere prospektiv randomisierte Studie zur Frage des differenziellen Einsatzes verschiedener Rekonstruktionen [622] zeigte, dass bei $74 \%$ der Patienten die Anlage eines J-Pouch technisch machbar ist. Verglichen mit einer transversen Coloplastik war in dieser Studie der J-Pouch hinsichtlich Stuhlfrequenz und Inkontinenzscore besser. Eine Metaanalyse unter Einschluss der FazioStudie relativierte zwar die Aussage zur Stuhlfrequenz, berücksichtigte aber weder die Langzeitergebnisse der Fazio-Studie, noch die Daten zur Inkontinenz [623]. Zur endgültigen Beurteilung des Stellenwerts der Seit-zu-End-Anastomose fehlen noch Studien mit ausreichender Fallzahl [624].

\subsubsection{Entscheidung zum Sphinkter-Erhalt}

\subsection{Konsensbasierte Empfehlung (2013)}

Durch die neoadjuvante Radiochemotherapie und entsprechende Remission kann u.U. trotz primär nicht für möglich gehaltenen Sphinktererhalts eine Schließmuskel erhaltende Rektumresektion möglich werden. Deshalb sollte diesbezüglich frühestens 6 Wochen nach Abschluss der Radiochemotherapie eine Reevaluation stattfinden.

GCP, starker Konsens

\section{Hintergrund}

Eine Reduktion der Tumormasse vor allem bei tiefsitzenden Tumoren ist mit entscheidend für den Erhalt des Sphinkterapparats. Hier zeichnet sich ein Vorteil für die neoadjuvante Radiochemotherapie $a b$, deren Effekte aber oft verzögert erst nach mehreren Wochen fassbar werden. Dies bedeutet, dass die Entscheidung für das zu wählende Operationsverfahren erst zum Zeitpunkt der Operation nach der erfolgten neoadjuvanten Radiochemotherapie getroffen werden darf [626]. Bildgebende Verfahren sind in der Beurteilung des Responses wenig hilfreich [627].

7.5.3.6. Vorgehen bei komplettem Response nach neoadjuvanter Therapie

\subsection{Konsensbasierte Empfehlung (2013)}

In den seltenen Fällen, in denen nach neoadjuvanter Radiochemotherapie klinisch, endoskopisch und durch bildgebende Verfahren (Endosonografie und MRT, alternativ evtl. auch CT) kein Tumor mehr nachweisbar ist, kann auf jegliche Operation verzichtet werden. Voraussetzung ist die gründliche Aufklärung über die noch unzureichende Validierung dieses Vorgehens und die Bereitschaft des Patienten, sich einer sehr engmaschigen mindestens 5-jährigen Nachsorge zu unterziehen.

GCP, Konsens

\section{Hintergrund}

In einer Studie aus Brasilien mit 265 Patienten mit Rektumkarzinom war bei $26,8 \%$ nach neoadjuvanter Therapie kein Tumor mehr nachweisbar. Diese Patienten wurden nicht operiert und erhielten eine Nachsorge [628]. Nach einem medianen Followup von 57,3 Monaten wurden bei 2 Patienten (2,8\%) ein endoluminales Rezidiv und bei 3 Patienten $(4,8 \%)$ systemische Metastasen nachgewiesen. Die Autoren postulieren, dass bei Patienten mit komplettem Ansprechen nach neoadjuvanter Radiochemo- 
therapie eine alleinige Nachsorge ohne Operation ausreichend zu sein scheint. Es ist jedoch zu bedenken, dass es sich um keine randomisierte Studie handelt und nicht abschließend geklärt ist, ob die Ergebnisse auch außerhalb von Brasilien erreicht werden können.

\subsubsection{Rektum-Exstirpation \\ 7.37. Empfehlung (2013)}

Bei tiefliegenden Tumoren mit Infiltration des Analkanals/der Sphinkteren, die nicht sphinktererhaltend operiert werden können, sollte die abdomino-perineale Exstirpation im Sinne einer „zylindrischen" Resektion unter Mitresektion des Levator ani erfolgen.

Empfehlungsgrad B, Level of Evidence 3b, de novo: [584, 629, 630], Konsens

\section{Hintergrund}

Bei der Rektum-Exstirpation ist klassisch die Rate an Präparaten mit unzureichenden lateralen Sicherheitsabständen erhöht [629]. Die onkologischen Ergebnisse sind gegenüber sphinktererhaltenden Eingriffen bei vergleichbaren Tumorstadien schlechter [630]. Die extralevatorische „zylindrische“ Resektion führt zu besseren Sicherheitsabständen und muss auch ohne Beweis durch randomisierte Studien als das überlegene Verfahren angesehen werden [584].

\subsection{Empfehlung (2013)}

Nach neoadjuvanter Radio- (Chemo-)-Therapie und/oder bei großen perinealen Defekten können die Wundheilungsstörungen perineal durch primäre plastische Deckung mit einem myokutanen Lappen reduziert werden.

Empfehlungsgrad O, Level of Evidence 3b, de novo: [631], Konsens

\section{Hintergrund}

Die Rate perinealer Wundheilungsstörungen ist insbesondere nach neoadjuvanter Bestrahlung und bei größeren Defekten hoch. Die Anwendung verschiedenen plastischen Rekonstruktionsverfahren bleibt aber eine Einzelfallentscheidung, bei der die individuelle anatomische Verfügbarkeit, die in Kauf zu nehmende Sekundärmorbidität des Hebedefekts, der operative Aufwand und die verfügbare chirurgische Expertise einzubeziehen sind [631].

\subsubsection{Stoma-Anlage}

7.39. Konsensbasierte Empfehlung/konsensbasiertes Statement (2013)

Bei der radikalen Operation des Rektumkarzinoms mit TME und tiefer Anastomose soll ein temporäres Deviations-Stoma vorgeschaltet werden.

GCP, starker Konsens

\subsection{Empfehlung (2013)}

Als Deviationsstoma sind Kolostoma und Ileostoma gleichwertig. GCP, starker Konsens

\section{Hintergrund}

Durch eine protektive Stomaanalage kann die Morbidität, insbesondere hinsichtlich klinisch relevanter Anastomoseninsuffizienzen und dringlicher Relaparotomien gesenkt werden [632]. Auch ist langfristig bei primärem Verzicht auf ein Deviationsstoma die Rate permanenter Stomata keineswegs geringer [633]. Zur Art des protektiven Stomas gibt es Argumente für beide Varianten, auch wenn jüngere Metaanalysen eher das Ileostoma favorisieren $[634,635]$.

\subsection{Konsensbasierte Empfehlung (2013)}

Die Stomaanlage soll möglichst frühzeitig vor der Operation mit dem Patienten besprochen und geplant werden.

GCP, starker Konsens

\subsection{Empfehlung (2013)}

Die Stomaposition soll präoperativ angezeichnet werden. GCP, Konsens

\subsection{Empfehlung (2013)}

Die Ileostomie sollte prominent angelegt werden (>1 cm). Die Kolostomie sollte leicht erhaben angelegt werden. GCP, starker Konsens

\section{Hintergrund}

Die präoperativen Informationen zur Stomaanlage sollten vom behandelnden Arzt und einer entsprechend ausgebildeten Pflegekraft (Stomatherapeut) gegeben werden. Ein Gespräch mit einem Betroffenen aus einer Selbsthilfegruppe sollte - sofern verfügbar - angeboten werden. Die Markierung des Stomas kann durch eine geschulte Pflegekraft/Stomatherapeut erfolgen, die Verantwortung für die korrekte Stomamarkierung und damit auch korrekte Anlage des Stomas liegt aber beim Arzt. Für eine optimale Lage ist es erforderlich, dass die Stomaposition im Liegen, Sitzen und Stehen angezeichnet wird.

Die postoperative Stomatherapie sollte sicherstellen, dass der Patient bzw. - sofern er selbst dazu nicht in der Lage ist - dessen Angehörige oder betreuende Personen, selbstständig die Stomaversorgung durchführen können (Grundplattenwechsel, Stomabeutel leeren und wechseln), die Versorgung mit Stomaartikeln gewährleistet ist und im Bedarfsfall der Zugang zu einem(r) Stomatherapeuten gewährleistet ist. Die Irrigation sollte Kolostomieträgern angeboten werden. Durch die präoperative Stomamarkierung und Einsatz von Stomatherapeuten konnte in einzelnen Studien eine Senkung der postoperativen Stoma-Komplikationsrate gezeigt werden [636, 637]. Des Weiteren konnte in einer prospektiven Studie gezeigt werden, dass die präoperative Stomaberatung die postoperative Versorgung vereinfacht [638].

\subsubsection{Lokale Operationsverfahren des Rektumkarzinoms 7.44. Statement (2008)}

Eine lokale chirurgische Tumorexzision beim Rektumkarzinom (Vollwandexzision) ist als alleinige therapeutische Maßnahme unter kurativer Zielsetzung onkologisch ausreichend bei pT1-Karzinomen mit einem Durchmesser bis zu $3 \mathrm{~cm}$, guter oder mäßiger Differenzierung, ohne Lymphgefäßinvasion (Low-risk-Histologie), sofern die Entfernung komplett erfolgt ist (RO). Level of Evidence 1b, [639-642], starker Konsens

\section{Hintergrund}

Auch in Low-risk-Fällen ist im Vergleich zur radikalen Operation mit einem höheren Lokalrezidiv-Risiko zu rechnen bei gleichzeitig geringerer Morbidität und Letalität und besserem funktionellen Ergebnis, sodass im Einzelfall diese Risiken gegeneinander abzuwägen sind [643, 644]. Es spricht vieles dafür, dass für die lokale Exzision die Technik der transanalen endoskopischen Mikrochirurgie der offenen transanalen Exzision mittels Spreizer überlegen ist [645, 646]. 
Hinsichtlich des Stellenwerts der sm-Klassifikation für die Einschätzung in „low-risk“ oder „high-risk“ konnte kein Konsens gefunden werden. T1-Karzinome mit tiefer submukosaler Infiltration (sm3, nach manchen Serien sogar sm2) werden von anderen Autoren und Leitlinien als Hochrisiko-Konstellation eingeschätzt, die einer radikalen Operation zugeführt werden sollte [647-650].

\subsection{Empfehlung (2008)}

Bei T1-High-risk-Karzinomen (G3/4 u./o. Lymphgefäßinvasion) und bei T2-Karzinomen liegt das Auftreten von Lymphknotenmetastasen bei 10-20\%, sodass die alleinige lokale Exzision nicht empfohlen werden kann.

(s. auch Abschnitt 6.4)

Empfehlungsgrad B, Level of Evidence 3b, [647, 651], starker Konsens

\section{Hintergrund}

Ist die „High-risk“-Konstellation prätherapeutisch bekannt, sollte primär radikal operiert werden. Stellt sich erst nach transanaler Vollwandresektion eine Hoch-Risiko-Konstellation heraus, so ist die sekundäre radikale Nachoperation innerhalb eines Monats nicht mit einer Verschlechterung der Prognose im Vergleich zum primär radikalen Vorgehen assoziiert [647, 651]. Lehnt der Patient in dieser Situation eine radikale Nachoperation ab, so kann eine adjuvante Radiochemotherapie erwogen werden.

\subsection{Laparoskopische Chirurgie \\ 7.46. Empfehlung (2013)}

Die laparoskopische Resektion des Kolon- und Rektumkarzinoms kann bei entsprechender Expertise des Operateurs und geeigneter Selektion mit gleichen onkologischen Ergebnissen im Vergleich zur offenen OP-Technik durchgeführt werden. ${ }^{5}$

Empfehlungsgrad A, Level of Evidence 1a, de novo: [652 -656], starker Konsens

\subsection{Konsensbasierte Empfehlung (2013)}

Die Qualität des Präparats soll durch den Pathologen dokumentiert werden.

GCP, starker Konsens

\section{Hintergrund}

Auch wenn laparoskopische kolorektale Resektionen längere Operationszeiten bedingen, sprechen zahlreiche randomisierte Studien dafür, dass im Kurzzeitverlauf die perioperative chirurgische Morbidität niedriger ist als nach konventioneller Operation bei unveränderter Gesamt-Morbidität und Letalität [652]. Im Langzeitverlauf konnten weder für die Rate an Narbenhernien und an adhäsions-bedingten Reoperationen noch für regionäre und systemische Tumorrezidive Unterschiede gefunden werden [653, 654]. Die systematischen Reviews und Metaanalysen zeigten äquivalente onkologische Langzeitergebnisse vor allem beim Kolonkarzinom, während die Datenlage zum Rektumkarzinom noch zu wünschen übrig ließ. Inzwischen wurden aber reife Langzeitergebnissen der britischen CLASICC-Studie publiziert, die trotz initial problematischer Surrogat-Parameter in der Untergruppe der Rektumkarzinome [655] die onkologische Sicherheit der laparoskopischen Chirurgie sowohl für das Kolon- als

\footnotetext{
${ }^{5}$ Das „kann“ im Empfehlungstext steht nicht im Widerspruch zum Empfehlungsgrad A, da sich dieser auf die Gleichwertigkeit der Verfahren bezieht.
}

auch das Rektumkarzinom belegen konnten [656]. Für die ohne Zweifel notwendige spezielle laparoskopische Expertise sind bisher keine stringenten Kriterien validiert.

\subsection{Konsensbasierte Empfehlung (2013)}

Neuere Operationsverfahren (z.B. Robotik, NOTES) können wegen unzureichender Daten außerhalb von Studien nicht empfohlen werden.

GCP, starker Konsens

\section{Hintergrund}

Das DaVinci-Telemanipulationssystem - der einzige in der klinischen Routine etablierte „Roboter“ - ist in mehreren Serien zur laparoskopischen total mesorektalen Exzision verwendet worden $[657,658]$. Da weder kurzfristige Vorteile noch die längerfristige onkologische Gleichwertigkeit bewiesen sind, ist diese Methode bisher als experimentell anzusehen. Gleiches gilt in noch stärkerem Maße für transgastrale, transvaginale oder transanale Zugänge und Präparate-Bergungen.

\subsection{Sondersituationen}

\subsubsection{Chirurgische Therapie der Peritonealkarzinose}

\subsection{Empfehlung (2013)}

Bei Patienten mit einer isolierten und limitierten Peritonealkarzinose kann eine zytoreduktive Chirurgie gefolgt von einer hyperthermen intraperitonealen Chemotherapie (HIPEC) durchgeführt werden, wenn folgenden Voraussetzungen erfüllt sind:

- $P C I$ (peritoneal cancer index) $<20$

- keine extraabdominellen Metastasen

- Möglichkeit der makroskopisch kompletten Entfernung oder Destruktion jeglicher Tumormanifestation

- Therapie in einem spezialisierten Zentrum

Die Durchführung im Rahmen von Studien sollte bevorzugt werden.

Empfehlungsgrad 0, Level of Evidence 2a, de novo: [659-662], starker Konsens

\section{Hintergrund}

Es existiert hierzu bisher nur eine einzige abgeschlossene randomisierte Studie zum Vergleich zwischen systemischer Chemotherapie und zytoreduktiver Chirurgie mit HIPEC [660]. Diese Studie konnte auch im Langzeitverlauf belegen, dass die chirurgische Therapie der Peritonealkarzinose zu signifikant besserem krankheits-spezifischem Überleben führt [661]. Ließ sich makroskopische Tumorfreiheit erzielen, lag das 5-Jahresüberleben bei $45 \%$. Einschränkend muss angeführt werden, dass der Kontrollarm lediglich eine 5-FU-basierte systemische Therapie erhielt. Trotz zahlreicher nicht randomisierter Studien mit ermutigenden Ergebnissen [662] ist damit nicht eindeutig definiert, welche Patienten über eine moderne systemische Polychemotherapie hinaus von einer chirurgischen Therapie mit HIPEC profitieren.

\subsubsection{Multiviszerale Resektion}

Bei Adhärenz eines Tumors an Nachbarorganen ist makroskopisch nicht sicher zu klären, ob es sich um eine Infiltration des Karzinoms in das Nachbarorgan oder nur um eine peritumoröse Entzündungsreaktion handelt. In solchen Fällen sollten Biopsien und Schnellschnittuntersuchungen strikt vermieden werden, da hierbei stets die Gefahr einer örtlichen Tumorzelldissemination besteht, was mit einer signifikanten Verringerung der Überlebenschancen einhergeht [571]. Daher sollte eine En-bloc-Resektion der befallenen Organe durchgeführt werden (multiviszerale Re- 
sektion). Im Falle des Rektumkarzinoms können totale Beckenexenterationen notwendig werden.

\subsubsection{Mehrfachkarzinome des Kolorektums}

In diesen Fällen sollte keine regelhafte Kolektomie erfolgen, sondern das Vorgehen unter Berücksichtigung der Erfordernisse der einzelnen Karzinome erfolgen, d. h. auch u.U. die Anlage mehrerer Anastomosen.

\subsubsection{Notfalloperationen}

Bei Ileus, Tumorperforation oder Darmperforation bei stenosierendem Tumor ist das Vorgehen abhängig von der vorliegenden Situation. Nach Möglichkeit ist eine onkologische radikale Resektion entsprechend dem elektiven Vorgehen anzustreben. In geeignet erscheinenden Fällen kann bei einem Ileus die Einlage eines endoluminalen Stents diskutiert werden [663]. Ein Ileus in Verbindung mit einem Rektumkarzinom geht in der Regel mit weit fortgeschrittenen Karzinomen einher, sodass fast immer eine neoadjuvante Radio-/Chemotherapie anzustreben ist. Aus diesem Grund wird bei dieser Situation häufig primär ein Transversostoma rechts angelegt. Eine tumorbedingte Blutung ist sehr selten relevant für einen Therapieentscheid.

\subsubsection{Resektion von Lebermetastasen}

7.50. Konsensbasiertes Statement (2013)

Die simultane Resektion von Lebermetastasen beeinflusst wahrscheinlich das Langzeitüberleben im Vergleich zu einem zweizeitigen Vorgehen bei geeigneter Selektion der Patienten nicht.

GCP, starker Konsens

\subsection{Empfehlung (2013)}

Die simultane Lebermetastasenresektion kann bei entsprechender Komorbidität oder höherem Lebensalter (> 70 Jahre) zu einer höheren postoperativen Letalität führen.

GCP, Konsens

\subsection{Empfehlung (2013)}

Bei multiplen synchronen Lebermetastasen sollte ein zweizeitiges und multimodales Vorgehen gewählt werden.

GCP, Konsens

\section{Hintergrund}

Die Entscheidung zur Simultanresektion von Lebermetastasen in Verbindung mit Resektion des Primärtumors hat mehrere Gesichtspunkte zu bedenken:

- Bei rechtsseitigen Kolonresektionen ist der Zugang zur Leber aufgrund der Lagerung des Patienten und der Schnittführung (abgesehen von sehr adipösen Patienten, einer großen Fettleber) in der Regel ohne Schwierigkeiten möglich. Bei linksseitigen Kolon- und Rektumoperationen und der hierfür üblicherweise gewählten Lagerung mit Erhöhung des Beckens gestaltet sich der Zugang zur Leber sehr viel schwieriger.

- Periphere Einzelmetastasen können auch bei der Lagerung für linksseitige Resektionen ohne wesentliche Erschwernisse durchgeführt werden, vor allen Dingen formale rechtsseitige Resektionen (z.B. Hemihepatektomie re.) gestalten sich nur bei rechtsseitigen Resektionen aufgrund des damit verbundenen Zugangs akzeptabel.

- Gerade kleine, einzelne Metastasen sind häufig von einer weitergehenden metachronen Metastasierung gefolgt, sodass es auch gerechtfertigt ist, trotz gegebener technischer Resektabilität die weitere Entwicklung bez. einer möglichen weiterge- henden Lebermetastasierung abzuwarten und evtl. eine systemische Chemotherapie zwischenzuschalten.

Als weiteres wird von einzelnen Chirurgen das Konzept der primären Leberresektion ohne jegliche Resektion des Primärtumors oder vorgeschaltete weitere Maßnahmen wie neoadjuvante Chemotherapie bzw. neoadjuvante Radiochemotherapie beim Rektumkarzinom vertreten.

\subsubsection{Ausgedehnte Fernmetastasierung und asymptomatischer Primärtumor \\ 7.53. Empfehlung (2013)}

Bei Patienten im Stadium IV mit ausgedehnter Lebermetastasierung („Metastasenleber“) und asymptomatischem Primärtumor (keine Stenosesymptomatik, keine transfusionspflichtigen Blutungen) kann ohne Resektion des Primärtumors primär eine Chemotherapie durchgeführt werden.

Empfehlungsgrad 0, Level of Evidence 4, de novo: [664, 665], starker Konsens

\section{Hintergrund}

Bei Patienten mit ausgedehnter irresektabler Fernmetastasierung sind in der Regel die Fernmetastasen prognosebestimmend. Besteht eine durch den Primärtumor bedingte Ileussymptomatik oder eine transfusionspflichtige Blutung ist eine zeitnahe Resektion des Primärtumors bis auf wenige Ausnahmen unerlässlich. Bei einem asymptomatischen Primärtumor ist jedoch unklar, ob eine Resektion für den Patienten einen Vorteil hat. So wird durch die Operation der Beginn einer Chemotherapie hinausgezögert. In einer Metaanalyse von 8 retrospektiven deskriptiven Studien (Zeitraum 1985 - 2005) fand sich in 7 der Studien ein verlängertes Überleben für Patienten mit Resektion des Primärtumors [664]. Die Studien waren jedoch nicht randomisiert d.h. die Gründe für eine Allokation von Patienten in die primäre OPoder primäre Chemotherapiegruppe bleiben unklar. Auch erscheint ein medianes Überleben von 6 Monaten in einigen Chemotherapiegruppen deutlich geringer, als mit den heute zur Verfügung stehenden Chemotherapieprotokollen erreichbar. Die Gefahr, dass bei primärer Chemotherapie im Verlauf eine Operation aufgrund eines Ileus oder Perforation erforderlich wird, ist nach der derzeitigen Datenlage gering (etwa 7\%) [665]. Die Einleitung einer Chemotherapie mit Belassen eines asymptomatischen Primärtumors bei ausgedehnter Fernmetastasierung erscheint daher gerechtfertigt. Der Stellenwert einer Resektion des Primärtumors bei ausgedehnter Fernmetastasierung wird derzeit im Rahmen einer randomisierten Studie (Synchronous-Studie, Studiennummer ISRCTN30 964555) untersucht.

\subsubsection{Karzinome auf dem Boden eines hereditären kolorektalen Karzinoms ohne Polyposis (HNPCC) \\ 7.54. Konsensbasierte Empfehlung (2013)}

Eine prophylaktische Kolektomie bzw. Proktokolektomie bei HNPCC-Mutationsträgern soll nicht durchgeführt werden.

Eine subtotale Kolektomie bei Karzinom sollte nicht generell durchgeführt, aber individuell mit dem Patienten besprochen werden. GCP, starker Konsens

\subsection{Empfehlung (2008)}

Die koloskopische Überwachung des Patienten nach onkologischer Resektion muss zusätzlich zur üblichen Nachsorge auch postoperativ nach dem gleichen Muster wie vor der Operation fortgesetzt werden (s. auch Kapitel 5.2.2.1). 
Empfehlungsgrad A, Level of Evidence 2a, Evidenz aus Aktualisierungsrecherche: [272, 273, 287 - 290], starker Konsens

\section{Hintergrund}

Da durch regelmäßige Vorsorgeuntersuchungen Karzinome bei fast allen Patienten im Stadium UICC I/II oder sogar als prämaligne Adenome entdeckt werden $[272,273]$ und die Penetranz unvollständig ist, wird von einer prophylaktische Kolektomie bzw. Proktokolektomie abgeraten.

Bei Nachweis eines Karzinoms werden die Patienten nach tumorchirurgischen Gesichtspunkten im Sinne einer onkologischen Resektion operiert. Das Risiko eines kolorektalen Karzinoms im verbliebenen Dickdarm und das Risiko von extrakolischen Neoplasien bleiben jedoch erhöht, sodass diese Patienten einer intensiven postoperativen Nachsorge zugeführt werden müssen. In dieser sollte die Tumornachsorge für sporadische KRK mit dem HNPCC-spezifischen Früherkennungsprogramm für KRK und extrakolische Tumoren kombiniert werden. Ob eine erweiterte prophylaktische Resektion zur Prophylaxe metachroner KRK einer engmaschigen Überwachung überlegen ist, ist derzeit unklar. Die bisherigen Daten aus retrospektiven Fallserien sind unzureichend, und aufgrund nationaler Unterschiede in Bezug auf das Screening-Intervall nicht auf Deutschland übertragbar (für weitere Informationen s. Kapitel 5.2.2.1).

\subsubsection{Karzinome auf dem Boden einer familiären} adenomatösen Polyposis (FAP)

\subsection{Empfehlung (2008)}

Die Regeloperation bei FAP-Patienten ist die restaurative Proktokolektomie mit Dünndarm-Pouch und Lymphknotendissektion entsprechend der Lokalisation des Karzinoms mit den sich hieraus ergebenen Behandlungserfordernissen (z.B. radikuläre Gefäßdurchtrennung, totale mesorektale Exzision). In Abhängigkeit von einer eventuellen Sphinkterinsuffizienz oder nicht kurablen Tumorerkrankungen kann auch eine Prokto-Kolektomie oder eine limitierte Resektion durchgeführt werden.

Empfehlungsgrad B, Bei einer attenuierten FAP mit diskretem Rektumbefall ist eine Ileorektostomie zu empfehlen (s. auch 5.2.2.2). Level of Evidence 3b [305] starker Konsens

\subsubsection{Karzinome auf dem Boden einer Colitis ulcerosa 7.57. Statement (2008)}

Die Regeloperation stellt die restaurative Prokto-Kolektomie mit Dünndarm-Pouch dar, falls aus onkologischen und funktionellen Gründen sinnvoll.

Level of Evidence 3b, starker Konsens

Für weitere Empfehlungen und Informationen z. B. zu Durchführungsbedingungen bei Überwachungskoloskopien, sei auf die S3Leitlinie Diagnostik und Therapie der Colitis ulcerosa [396] verwiesen.

\subsection{Postoperative pathohistologische Diagnostik 7.58. Empfehlung (2008) \\ Folgende Angaben durch den Pathologen sind erforderlich: \\ - Tumortyp nach WHO-Klassifikation \\ Empfehlungsgrad A, Level of Evidence 1c \\ - Tumorinvasionstiefe (pT-Klassifikation) \\ Empfehlungsgrad A, Level of Evidence 1c \\ - Status der regionären Lymphknoten ( $\mathrm{pN}$-Klassifikation) \\ Empfehlungsgrad A, Level of Evidence 1c \\ - Anzahl der untersuchten Lymphknoten \\ Empfehlungsgrad A, Level of Evidence 2a}

- Mindestanzahl der zu untersuchenden Lymphknoten: 12

Empfehlungsgrad A, Level of Evidence 2a

- Grading

Empfehlungsgrad A, Level of Evidence 2a

- Abstand von den Resektionsrändern (beim Rektumkarzinom auch zirkumferenziell)

Empfehlungsgrad A, Level of Evidence 2a

- R-Klassifikation

Empfehlungsgrad A, Level of Evidence 1c

Quellen für alle Angaben: [477, 579, 666 - 678]

\section{Hintergrund}

Zunehmend wird vor allen Dingen nach neoadjuvanter Radiochemotherapie das Ausmaß der eingetretenen pathohistologisch fassbaren Remission in Anlehnung an Dworak klassifiziert [678].

\subsection{Konsensbasierte Empfehlung (2008)}

Die Untersuchung auf Mikrosatelliteninstabilität erfolgt fakultativ bei V.a. Vorliegen eines HNPCC.

GCP

\subsubsection{Karzinom-Graduierung in Abhängigkeit von MSI-H}

\subsection{Konsensbasierte Empfehlung (2013)}

Schlecht differenzierte Adenokarzinome einschließlich muzinöser Adenokarzinome und undifferenzierte Karzinome sollen immunhistochemisch auf die Expression von hMLH1 und hMSH2 getestet und bei einem Ausfall von hMLH1 oder hMSH2 als niedriggradige (low grade) Karzinome graduiert werden.

GCP, Konsens

\section{Hintergrund}

Kolorektale Karzinome mit hochgradiger Mikrosatelliteninstabilität (MSI-H) haben eine signifikant günstigere Prognose und niedrigere Fernmetastasierungsrate als kolorektale Karzinome mit Mikrosatellitenstabilität (MSS) oder niedriggradiger Mikrosatelliteninstabilität (MSI-L) [679-682]. Auch wurde gezeigt, dass wenig differenzierte (G3) Adenokarzinome, aber auch muzinöse Adenokarzinome, die bislang als G3 graduiert wurden, bei Vorliegen von MSI-H eine signifikant günstigere Prognose haben als bei Vorliegen von MSS oder MSI-L [683, 684]. Daher sollen wenig differenzierte Adenokarzinome einschließlich der muzinösen Adenokarzinome und undifferenzierte Karzinome im Dickdarm nicht als hochgradig (high grade), sondern als niedriggradig (low grade) eingestuft werden, wenn Hinweise für MSI-H bestehen.

Der immunhistochemische Nachweis eines Expressionsausfalls für hMLH1 bzw. hMSH2 hat eine vergleichbare prognostische Relevanz wie MSI-H [685] und kann aufgrund seiner Sensitivität und Spezifität als einfacher und kostengünstiger Ersatz für eine molekulare MSI-Testung zur MSI-assoziierten Prognosebewertung eingesetzt werden [686-689]. Die Immunhistochemie für hMLH1 und hMSH2 erfasst die sporadischen MSI-H-Karzinome und einen Teil der MSI-H-Karzinome bei Lynch-Syndrom. Der Zusammenhang eines kolorektalen Karzinoms mit dem Lynch-Syndrom kann hierdurch nicht zuverlässig bewertet werden und erfordert zusätzliche Analysen. 


\subsubsection{Anzahl zu entfernender LK}

7.61. Empfehlung (2013)

12 und mehr Lymphknoten sollen entfernt und untersucht werden. GCP, starker Konsens

7.62. Empfehlung 2013

Das Verhältnis von untersuchten zu befallenen Lymphknoten sollte angegeben werden.

GCP, starker Konsens

\subsection{Konsensbasiertes Statement (2013)}

Je größer die Zahl der entfernten und untersuchten Lymphknoten, desto besser ist die Prognose der Patienten mit KRK im UICC-Stadium II und III.

Die Zahl der entfernten und untersuchten Lymphknoten kann als Surrogatmarker für Behandlungsqualität gelten.

Die Größe des Lymphknotens korreliert nicht mit der Wahrscheinlichkeit einer Metastase.

GCP, starker Konsens

\section{Hintergrund}

Die Zahl der entfernten bzw. untersuchten Lymphknoten hängt nicht nur von der Tumorbiologie, sondern auch vom Chirurgen und Pathologen ab. Die 7. Auflage der UICC-TNM-Klassifikation 2010 gibt vor, dass für die Kategorie „pN0“ eine regionäre Lymphadenektomie und histologische Untersuchung von üblicherweise zwölf und mehr Lymphknoten erfolgen sollte.

Obwohl die Studienqualität zur Zahl der Lymphknoten gering ist, gilt jedoch, dass Patienten mit einer größeren Zahl entfernter und untersuchter Lymphknoten eine verbesserte Prognose im UICC-Stadium II und III aufweisen. Dier Zusammenhang ist in Kohortenstudien immer wieder gezeigt worden, so an 3411 Patienten im Stadium II und III des sog. Intergroup-Trials [579]. Die sog. INTACC-Studie an 3491 Patienten konnte ebenfalls zeigen, dass die Prognose mit der Zahl der entfernten/untersuchten Lymphknoten korreliert [690]. Dabei ist nicht nur die Zahl der Lymphknotenmetastasen relevant, sondern generell die der Lymphknoten. Beide Studien wiesen auch bei nodalnegativen Tumoren einen prognostischen Effekt nach, der zur Zahl der entfernten/ untersuchten Lymphknoten korrelierte [691].

Dieser Effekt wurde in zahlreichen Kohorten nachgewiesen [692 - 695].

Die Zahl der Lymphknoten kann als Surrogatmarker für die Behandlungs- und Diagnosequalität sowohl für die Chirurgie als auch Pathologie gelten.

Die „optimale Zahl“ der zu entfernenden/untersuchenden Lymphknoten für ein korrektes Staging wird in der Literatur kontrovers diskutiert; sicher ist allerdings, dass zwölf Lymphknoten nicht ausreichend sind. Die alleinige histopathologische Aufarbeitung der größten Lymphknoten am Präparat ist nicht ausreichend, da die Größe des Lymphknotens nicht mit der Wahrscheinlichkeit einer Metastase korreliert.

In der Literatur wird angeregt, das Verhältnis von untersuchten zu befallenen Lymphknoten anzugeben [696 - 698].

Bei Patienten, die eine präoperative Radiochemotherapie aufgrund eines lokal fortgeschrittenen Rektumkarzinoms erhalten haben, ist die Zahl der Lymphknoten geringer. Eine Analyse von 615 Patienten, die aufgrund eines primären Adenokarzinoms des Rektums operiert wurden, dass 33\% weniger Lymphknoten gefunden wurden, wenn präoperativ eine neoadjuvante Therapie durchgeführt wurde [699].

\subsubsection{Bedeutung des Tumorabstands zur Resektions-} fläche des Mesokolons beim Kolonkarzinom

7.64. Konsensbasierte Empfehlung (2013)

Analog der Qualitätsbewertung von Resektaten beim Rektumkarzinom sollte auch beim Kolonkarzinom die Qualität des Resektats folgendermaßen kategorisiert werden:

- Grad 1 (gut): Mesokolische Schicht erhalten

- Grad 2 (moderat): Oberflächeneinrisse

- Grad 3 (schlecht): Einrisse reichen bis auf die Muscularis propria oder Tumor

Diese Bewertungen sind vom Pathologen vorzunehmen.

GCP, Konsens

\section{Hintergrund}

Bezüglich des zirkumferenziellen Sicherheitsabstands des Primärtumors beim Kolonkarzinom liegen keine vergleichbaren Daten wie zum Rektumkarzinom vor. Zudem besteht bei Kolonkarzinomen sehr viel häufiger die Möglichkeit, trotz ausgedehnter Tiefeninfiltration des Primärtumors die Resektion angrenzender Strukturen oder Organe zu einem ausreichenden zirkumferenziellen Sicherheitsabstand zu kommen. In Ausnahmefällen ist dies nicht möglich (z. B. beim Sigmakarzinom, welches den Iliacalgefäßen aufsitzt).

Gegebenenfalls sollte deshalb analog zum Rektumkarzinom der zirkumferenzielle Sicherheitsabstand dokumentiert werden.

Zwischenzeitlich liegen jedoch Publikationen analog zum Rektumkarzinom über die Qualität der Bewertung von Kolonkarzinomresektaten vor. Ebenso wie beim Rektumkarzinom ist inzwischen belegt, dass Einrisse bis auf die Muscularis propria oder auf den Tumor zu einer schlechteren Überlebensrate führen (15\% geringere 5-Jahres-Überlebenswahrscheinlichkeit bei Muscularis propria vs. mesokolischer Schicht), wobei der Unterschied im Falle von Lymphknotenmetastasen (UICC-Stadium III) stärker ausgeprägt ist (27\% höhere Überlebenswahrscheinlichkeit nach 5 Jahren Followup), als bei fehlenden Lymphknotenmetastasen [700, 701].

\subsubsection{Bedeutung des Abstands vom zirkumferenziellen} Resektionsrand (CRM-Klassifikation) beim Kolon- und Rektumkarzinom

7.65. Konsensbasierte Empfehlung (2013)

Der zirkumferenzielle Sicherheitsabstand ist negativ, wenn er $1 \mathrm{~mm}$ oder mehr beträgt ( $R 0$ „wide“). Ein positiver zirkumferenzieller Sicherheitsabstand liegt vor, wenn der zirkumferenzielle Sicherheitsabstand weniger als $1 \mathrm{~mm}$ beträgt ( $R 0$ „close“) oder Tumorgewebe direkt an ihn heranreicht (R1). Der gemessene Abstand soll in Zahlen dokumentiert werden.

GCP, starker Konsens

\section{Hintergrund}

Nach den offiziellen Definitionen der AJCC wird der residuale Tumor als R1 definiert, wenn ein Karzinom tatsächlich histologisch bis an den Resektionsrand (RR) heranreicht ( $\bullet$ Abb. 4).

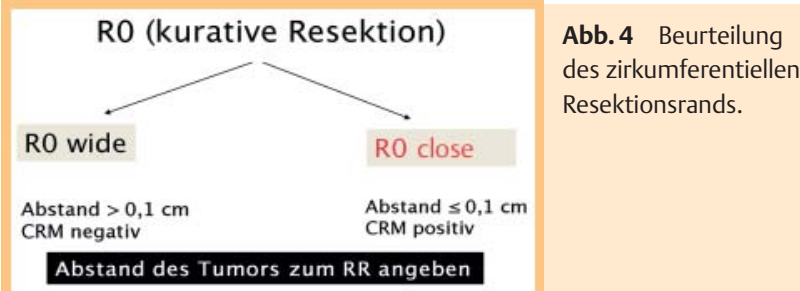

Berücksichtigt werden kontinuierliche Primärtumorausläufer, Satelliten, Lymphgefäß-/ Veneninvasion, Perineuralscheideninvasion, Lymphknotenmetastasen. 
Vor allem Arbeiten aus den Niederlanden und Großbritannien haben jedoch gezeigt, dass auch bei einem zirkumferenziellen Sicherheitsabstand von weniger als $1 \mathrm{~mm}$ das Lokalrezidivrisiko beim Rektumkarzinom deutlich ansteigt (3-Jahres-Lokalrezidivraten: $6 \%$ bei CRM- und $17 \%$ bei CRM+; 3-Jahres tumorfreies Überleben $79 \%$ bei CRM- und $50 \%$ bei CRM+ [560, 702].

Ob bereits ein Sicherheitsabstand von weniger als $2 \mathrm{~mm}$ zu einer verschlechternden Prognose führt, wird kontrovers diskutiert [703, 704].

\subsubsection{Bedeutung der Angabe der Qualität des TME-Präparats}

7.66. Konsensbasierte Empfehlung (2013)

Da die Qualität eines Operationsresektats unter Berücksichtigung der oben genannten Kategorien Rückschlüsse auf die Prognose bezüglich der Entwicklung eines Lokalrezidives zulässt, soll diese obligat im pathohistologischen Befundbericht wie folgend beschrieben werden:

Die Qualität des Präparats wird beurteilt an der Integrität der mesorektalen Faszie im Falle der Resektion mit 3 Kategorien:

- Grad1 (gut): Mesorektale Faszie erhalten

- Grad 2 (moderat): Intramesorektale Einrisse

- Grad 3 (schlecht): Erreichen der Muscularis propria oder Tumor Im Falle einer Rektumexstirpation sind bei einer vollständigen Resektion der Levatormuskulatur Präparateinrisse und ein tumorpositiver zirkumferenzieller Sicherheitsabstand seltener [705].

Im pathohistologischen Befundbericht ist deshalb die Beschreibung bezüglich der Radikalität im Bereich der Levatormuskulatur obligat. Hierzu sollen folgende Kategorien Verwendung finden:

- Grad 1 (gut): Levatormuskulatur mitreseziert, keine Eröffnung des Darmes oder des Tumors

- Grad 2 (moderat): Muscularis propria erhalten, keine Eröffnung des Darmes oder des Tumors

- Grad 3 (schlecht): Teile der Muscularis propria fehlen oder Eröffnung des Darmes oder des Tumors

Diese Bewertungen sind vom Pathologen vorzunehmen.

GCP, Konsens

\section{Hintergrund}

Die Qualität eines Rektumresektats hat signifikanten Einfluss auf die Lokalrezidivrate.

Bei intaktem Mesorektum betrug das 5-Jahres-tumorfreie Überleben $65 \%$ im Vergleich zu $47 \%$ bei Defekten des Mesorektums $(\mathrm{p}<0,05)$ [706].

Nach 3 Jahren Follow-up betrug die Lokalrezidivrate bei intakter mesorektaler Faszie 4\% (3-6\%), 7\% (5-11\%) bei intramesorektalen Einrissen, und 13\% (8 - 21\%) bei Erreichen der MuscularisPropria-Ebene [702].

Bei der Beurteilung eines Präparats nach einer Rektumexstirpation wird zwischen zylindrischer Exzision und Standardexzision unterschieden. Nach einer zylindrischen Exzision ist der zirkumferenzielle Resektionsrand signifikant seltener befallen und Perforationen des Präparats sind ebenfalls signifikant seltener. [700, 705]. Daten zur Auswirkung auf Lokalrezidivraten und Überlebensraten liegen bislang nicht vor.

Die Beurteilung der Qualität des chirurgischen Präparats sollte anhand der oben genannten Kriterien durch den Pathologen und nicht den Chirurgen erfolgen.

\subsection{Psychoonkologische Aspekte \\ 7.67. Empfehlung/GCP ${ }^{6}$ (2013)}

Psychoonkologische Behandlungsmaßnahmen sollten in das Gesamtkonzept der onkologischen Therapie integriert werden.

Empfehlungsgrad B, Level of Evidence 1b, [708-735]

\subsection{Empfehlung/GCP ${ }^{6}$ (2013)}

Alle Patienten sollten von ärztlicher Seite frühzeitig über Möglichkeiten psychoonkologischer Hilfestellungen informiert werden. GCP

\section{Hintergrund}

Über den gesamten Verlauf einer Krebserkrankung treten behandlungsbedürftige psychische Belastungen und Störungen mit einer Häufigkeit von 20-35\% (Krebspatienten aller Tumorlokalisationen und Krankheitsstadien) auf. Es überwiegen Anpassungsstörungen (F 43.12), akute Belastungsreaktionen (F 43.0) gefolgt von depressiven Störungen (Major Depression 8-20\%, Dysthymie 5-15\%) [710-713]. Für die Gruppe der Patienten mit kolorektalen Karzinomen liegen vergleichbare Zahlen vor [714, 715]. Fortgeschrittenes Krankheitsstadium, ausgeprägte funktionelle Beeinträchtigung und hoher somatischer Beschwerdedruck sind mit höherem Risiko psychischer Störungen verbunden [716]. Die zusätzliche Anlage eines Kolostoma bedeutet in der Regel eine eingreifende Veränderung für betroffene Patienten. Die Akzeptanz fällt umso schwerer, je beeinträchtigender die Funktionseinschränkungen sind und je massiver die körperliche Entstellung empfunden wird. Das Selbstwertgefühl der Patienten kann durch ein Stoma deutlich vermindert werden, sodass körperliche, sportliche und soziale Aktivitäten wie auch die Aufnahme der beruflichen Tätigkeit als schwierig und belastend erlebt werden und zu psychischen Beeinträchtigungen führen können. Gerade die äußerlich sichtbare Körperveränderung durch ein Stoma erschwert die Anpassung und führt zu Selbstwert- und Anpassungsstörungen bis hin zu einer Depression [715, 717]. Bei vielen Betroffenen werden Scham- und auch Ekelgefühle sowie Schmutz- und Geruchsängste zu einer großen psychischen Belastung, die das Bedürfnis nach Intimität gänzlich in den Hintergrund treten lassen. Insofern empfinden Stomaträger häufig ihr Sexualleben durch das Stoma negativ beeinflusst [718].

Ein beträchtlicher Prozentsatz psychischer Störungen bei Tumorpatienten wird nicht zutreffend diagnostiziert und bleibt unzureichend behandelt $[712,719,720]$ mit nachteiligen Auswirkungen auf körperliches Befinden, Funktionsstatus, Beschwerden (Schmerzen, Übelkeit, Fatigue) und die Lebensqualität der Patienten. Deshalb sollte das psychische Befinden der Patienten regelmäßig im Krankheitsverlauf, d.h. in allen Krisenphasen und zu Zeiten mit erwartbar hoher Belastung ermittelt werden. Neuere Studien sprechen für die Wirksamkeit von Vorsorge-/Früherkennungs-basierten psychosozialen Interventionen bei Tumorpatienten [711, 721, 722]. Vorsorge-/Früherkennungs-Verfahren beinhalten die Beantwortung einiger einfacher gezielter Fragen durch den Patienten, entweder im persönlichen Kontakt oder mithilfe eines Fragebogens. Verschiedene Screening-Verfahren stehen zur Identifizierung behandlungsbedürftiger Patienten

\footnotetext{
${ }^{6}$ Entnommen der Interdisziplinären S3-Leitlinie für die Diagnostik, Therapie und Nachsorge des Mammakarzinoms [707]. Kreienberg R. et al. Interdisziplinäre S3-Leitlinie für die Diagnostik, Therapie und Nachsorge des Mammakarzinoms. Informationszentrum für Standards in der Onkologie (ISTO), Deutsche Krebsgesellschaft e.V. 2008; 1 . Aktualisierung
} 
mit hoher psychischer Belastung bzw. Komorbidität zur Verfügung. Eine Übersicht mit Darstellung verschiedener Screeningverfahren findet sich bei [723], online erhältlich unter www. pso-ag.de.

Professionelle psychische Unterstützung/Mitbehandlung soll für alle Patienten und deren Angehörige verfügbar sein. Dies kann in Form eines psychoonkologischen, eines psychosomatisch oder psychiatrischen Konsil-/Liaison-Dienstes, durch psychoonkologische Fachkräfte in Organ- und onkologischen Zentren oder durch Einbeziehung niedergelassener ärztlicher oder psychologischer Psychotherapeuten mit psychoonkologischer Qualifizierung erfolgen [724-726]. Sie hat in enger Kooperation mit und Rückmeldung an die behandelnden Ärzte und Pflegekräfte zu erfolgen.

Alle Patienten mit kolorektalem Karzinom sollen von ihren medizinischen Behandlern (Ärzten/Pflegekräften) über die Verfügbarkeit professioneller psychischer Unterstützung bzw. Mitbehandlung informiert werden.

Die Wirksamkeit unterschiedlicher psychoedukativer und psychotherapeutischer Interventionen bei Tumorpatienten hinsichtlich Symptomreduktion (Depression, Angst, Schmerzen, Fatigue), Krankheitsverarbeitung und Verbesserung der Lebensqualität ist gesichert [709, 720, 727 - 735].

Weiterführend wird auf die S3-Leitlinie Psychoonkologie, Fertigstellung voraussichtlich 2013, hingewiesen.

\section{Adjuvante und neoadjuvante Therapie \\ $\nabla$}

8.1. Adjuvante Therapie des Kolonkarzinoms

8.1.1. Indikation zur adjuvanten Behandlung

bei Kolonkarzinomen

Voraussetzung für eine adjuvante Therapie ist die R0-Resektion des Primärtumors. Grundlage für die Indikation zur adjuvanten Therapie nach Tumorresektion ist die pathohistologische Stadienbestimmung, insbesondere die Bestimmung des pN-Status. Zur Festlegung von pN0 sollen 12 oder mehr regionäre Lymphknoten untersucht werden (UICC 2002). Immunzytologische Befunde von isolierten Tumorzellen in Knochenmarkbiopsien oder Lymphknoten sowie zytologische Tumorzellbefunde in Peritonealspülungen sind keine Indikation zur adjuvanten Therapie außerhalb von Studien.

Für Patienten mit einem kurativ resezierten Kolonkarzinom im Stadium I ist eine adjuvante Therapie nicht indiziert. Patienten des UICC-Stadiums II und III sollten möglichst in kontrollierte Studien eingebracht werden, um Aufschluss über die Indikationsstellung und die optimale adjuvante Therapie zu erhalten. Der Verlauf von Patienten, die außerhalb klinischer Studien behandelt werden, ist im Rahmen der Qualitätssicherung hinsichtlich des Auftretens von Rezidiven, der Überlebensrate und von Nebenwirkungen zu dokumentieren. Die Durchführung der adjuvanten Chemotherapie erfordert einschlägige Erfahrung und insbesondere die Kenntnis der entsprechenden Dosisreduktionsschemata, die bei auftretender Toxizität eingehalten werden müssen.

Kontraindikationen der adjuvanten Chemotherapie bei Kolonkarzinomen

- Allgemeinzustand schlechter als 2 (WHO)

- Unkontrollierte Infektion

- Leberzirrhose Child B und C

- Schwere koronare Herzkrankheit, Herzinsuffizienz (NYHA III und IV)
- Präterminale und terminale Niereninsuffizienz

- Eingeschränkte Knochenmarkfunktion

- Andere, die Lebenserwartung bestimmende Komorbiditäten

- Unvermögen, an regelmäßigen Kontrolluntersuchungen teilzunehmen

\subsubsection{Altersbeschränkung für die Durchführung} einer adjuvanten Chemotherapie

\subsection{Empfehlung (2008)}

Eine Altersbeschränkung für die Durchführung einer adjuvanten Chemotherapie existiert nicht, allgemeine Kontraindikationen (s. o.) sind zu berücksichtigen.

Empfehlungsgrad A, Level of Evidence 1, starker Konsens

\section{Hintergrund}

In randomisierten Studien zum Einfluss einer adjuvanten Chemotherapie bei Kolonkarzinomen waren ältere Patienten unterrepräsentiert. Eine Ursache war, dass in den meisten Studien eine Altersbeschränkung als Einschlusskriterium galt. Eine prospektive Kohortenstudie mit Patienten über 67 Jahren mit Kolonkarzinom zeigte auch bei diesen älteren Patienten einen signifikanten Überlebensvorteil durch eine adjuvante Chemotherapie im Vergleich zur alleinigen Operation [736]. Auch eine retrospektive Studie mit einer geringen Patientenanzahl zeigte, dass keine signifikanten Überlebenszeitdifferenzen in Abhängigkeit vom Alter vorhanden sind [737]*. Dieses Ergebnis wurde durch eine gepoolte Analyse von 7 Studien mit 500 Patienten über 70 Jahren bestätigt [738]. In dieser Studie war die Rate gastrointestinaler Nebenwirkungen nicht altersabhängig, es kam jedoch häufiger zur Leukopenie bei den älteren Patienten. In einer weiteren Studie trat als einzige Nebenwirkung eine Stomatitis häufiger in der Gruppe über 70 Jahre auf [739]. Die adjuvante Chemotherapie scheint demnach in den meisten Fällen auch von älteren Patienten gut vertragen zu werden. Auch der Nutzen einer zusätzlichen adjuvanten Therapie mit Oxaliplatin war in einer Subgruppenanalyse der MOSAIC-Studie nicht altersabhängig [740]. Das Lebensalter eines Patienten hat somit keinen alleinigen prädiktiven Wert [741].

\subsubsection{UICC-Stadium III}

\subsection{Empfehlung (2008)}

Bei Patienten mit einem R0-resezierten Kolonkarzinom im Stadium III ist eine adjuvante Chemotherapie indiziert.

Empfehlungsgrad A, Level of Evidence 1a, starker Konsens

\section{Hintergrund}

In zahlreichen randomisierten Studien konnte ein signifikanter Überlebensvorteil für Patienten mit Kolonkarzinom im Stadium III durch eine adjuvante Chemotherapie nachgewiesen werden [742, 743]. Metaanalysen und gepoolte Analysen (zuletzt Gill et al.) bei 3303 Patienten mit einem Stadium II und III Kolonkarzinom konnten eindeutig zeigen, dass für Patienten mit einer Lymphknoten positiven Erkrankung (Stadium III) die Durchführung einer adjuvanten Chemotherapie mit einer signifikanten Prognoseverbesserung im Vergleich zur alleinigen Operationen assoziiert ist [738, $744-746]$. 


\subsubsection{UICC-Stadium II}

\subsection{Empfehlung (2008)}

Bei Patienten mit einem kurativ resezierten Kolonkarzinom im Stadium II kann eine adjuvante Chemotherapie durchgeführt werden. Empfehlungsgrad 0, Level of Evidence 1b, starker Konsens

\section{Hintergrund}

Der absolute Nutzen einer adjuvanten Therapie im UICC-Stadium II ohne Risikofaktoren liegt absolut zwischen 2 - 5\%. Studien und gepoolte Analysen von Studien bei Patienten mit einem Kolonkarzinom im Stadium II fand sich kein signifikanter Überlebensvorteil durch eine postoperative adjuvante Chemotherapie [747 - 750]. Die gepoolte Analyse von 7 randomisierten Studien, die eine adjuvante Chemotherapie mit einer alleinigen Operation verglichen, zeigte in der univariaten Analyse nur eine signifikante Verbesserung für das 5-Jahres-krankheitsfreie Überleben (DFS) (72 vs. 76\%; $p=0,049$ ), aber nicht für das 5-Jahres-Gesamtüberleben ( 80 versus $81 \%$; $=0,1127$ ) im Stadium II, wobei sich die Einzelstudien deutlich in den Therapiemodi unterschieden und kleine Patientenzahlen einschlossen [745]. Die britische QUASAR-Studie ist die für diese Fragestellung größte publizierte Einzelstudie [751]. Hier war in einem Beobachtungszeitraum von (im Median) 5,5 Jahren das relative Risiko für einen Tod jedweder Ursache in der Therapiegruppesignifikant geringer als in der Beobachtungsgruppe (HR 0,82; $95 \% \mathrm{CI}: 0,70-0,95, \mathrm{p}=0,008$ ), entsprechend einem absoluten Überlebensvorteil von ca. $3 \%$ (95\% CI:1,0-6,0). Allerdings hat auch diese Studie methodische Schwächen hinsichtlich ihres heterogenen Studienkollektivs (71\% Kolonkarzinome, 91\% Dukes' B Stadien) und der heterogenen 5-FU-haltigen Therapieprotokolle (mit oder ohne Levamisol, Folinsäuredosierung unterschiedlich). Das Relative Risiko für die isolierte Subgruppe Kolonkarzinom im Stadium II war nicht signifikant verringert, allerdings war der Effekt in allen Subgruppen konsistent gleich groß, sodass ein Überlebensvorteil in allen Prognosegruppen angenommen werden kann. Hinsichtlich der Bedeutung dieser Studie für die so definierte „Hochrisikosituation" (s. u.) kann keine Empfehlung abgeleitet werden, da lediglich für ca. $20 \%$ der Patienten Daten für die T-Kategorie und/ oder eine vaskuläre Invasion vorliegen und von diesen $20 \%$ die wenigsten Patienten tatsächlich eine T4 oder V1- Situation aufwiesen. Für den Einsatz von Oxaliplatin im Stadium II liegen keine überzeugenden Daten vor: Auf dem ASCO 2007 [752]* wurde in einer Untergruppenanalyse über den Effekt einer adjuvanten postoperativen Chemotherapie (FOLFOX4 versus LV5FU2) im Stadium II berichtet. Für das Stadium II konnte weder eine signifikante Verbesserung des krankheitsfreien Überlebens (HR 0,84; $95 \%$ CI: 0,62-1,14; $\mathrm{p}=0,258)$ noch ein Überlebensvorteil (HR 1,0; $95 \%$ CI: 0,71 - 1,42) für die zusätzlich mit Oxaliplatin behandelten Patienten mit einem Stadium II Tumor belegt werden. Aus den bisher vorliegenden randomisierten kontrollierten Studien lässt sich somit ein obligater Einsatz einer adjuvanten Chemotherapie im Stadium II nicht ableiten [753 - 755]. Aufgrund der positiven Ergebnisse der bislang größten Untersuchung, der QUASAR Studie, kann jedoch - bei allen methodischen Mängeln - der Nutzen einer adjuvanten Therapie im Stadium II ohne Risikofaktoren nicht gänzlich ausgeschlossen werden. Deshalb sollte eine Therapie in diesem Stadium zumindest in Betracht gezogen werden [751], in jedem Fall sollten die Vorteile und Risiken einer solchen Therapie mit dem Patienten besprochen werden.

\subsubsection{UICC-Stadium II mit Risikofaktoren}

\subsection{Empfehlung (2008)}

Im Stadium II sollte in ausgewählten Risikosituationen (T4, Tumorperforation/-einriss, Operation unter Notfallbedingungen, Anzahl untersuchter Lymphknoten zu gering) eine adjuvante Chemotherapie erwogen werden.

Empfehlungsgrad B, Level of Evidence 3, starker Konsens

\section{Hintergrund}

Die oben genannten Faktoren sind als prognostisch ungünstig identifiziert worden. Es erscheint daher denkbar, dass Patienten mit diesen Risikofaktoren auch im Stadium II von einer adjuvanten Chemotherapie profitieren könnten. Es existieren jedoch keine prospektiven Daten zum Zusammenhang der aufgeführten Risikocharakteristika und dem Nutzen einer adjuvanten Chemotherapie. Daher sollen vor Einleitung einer adjuvanten Chemotherapie auch in dieser Untergruppe mit dem Patienten in einem intensiven Gespräch die Vor- bzw. Nachteile einer adjuvanten Chemotherapie vor dem Hintergrund der aktuellen Datenlage erörtert und die Patientenpräferenzen angesprochen werden.

In Studien gingen gewisse Risikosituationen wie ein T4-Tumor, eine Tumorperforation und/oder Operation unter Notfallbedingungen sowie eine zu geringe Anzahl untersuchter Lymphknoten mit einer schlechteren Prognose einher [756, 757]. Eine neuere retrospektive Untersuchung an 1306 Patienten mit einem Stadium-II-Tumor zeigte in einer multivariaten Analyse, dass die Kategorie T4 mit einem schlechten krankheitsfreien Überleben assoziiert war $(H R$ 1,75) [758]. In der Studie von Moertel $(n=318)$ besitzt die T4-Kategorie im Stadium II keine zusätzliche prognostische Bedeutung [753], wohl aber in der Studie von Burdy $(n=108)$ [759], in der Erlanger Analyse $(n=305)$ [756] und in der veröffentlichten Metaanalyse von Gill [745].

Nach einer Notfalloperation resultierte mit 29,8 vs. $52,4 \%$ eine signifikant niedrigere 5 -Jahres-Überlebensrate $(\mathrm{p}<0,001)$. Dieser Unterschied zeigte sich sowohl im Stadium I/II als auch im Stadium III [760]. Das karzinomspezifische Überleben nach 5 Jahren reduzierte sich von $74,6 \%$ auf $60,9 \%$ bei Nachweis einer Anämie, auf $51,6 \%$ bei Nachweis einer Stenose und auf $46,5 \%$ bei Perforation ( $p<0,001$ ) [761]. Auch die Anzahl der untersuchten Lymphknoten galt in mehreren Studien als ein unabhängiger prognostischer Faktor [579, 762]. So betrug bei 222 Patienten mit einem KRK im Stadium II die 5-Jahres-Überlebensrate 49\% für Patienten, bei denen 6 oder weniger als 6 Lymphknoten untersucht worden waren, verglichen mit $68 \%$ für Patienten mit 7 oder mehr untersuchten Lymphknoten [762]. Le Voyer (INT-0089, $\mathrm{n}=3411$ ) untersuchte Patienten im Stadium Dukes B2 oder Dukes $\mathrm{C}$ mit adjuvanter Therapie mit 5-FU, Folinsäure (FS) und/oder Levamisol. Sowohl bei N0, N1 als auch N2 hatte die Anzahl der entfernten Lymphknoten eine prognostische Bedeutung. Patienten mit N0-Tumoren hatten das beste Gesamtüberleben, wenn mehr als 20 Lymphknoten analysiert wurden [579]. In einer Untersuchung von 3592 Fällen mit kolorektalem Karzinom konnte eine englische Arbeitsgruppe [763] jeweils einen signifikanten Überlebensvorteil für Patienten mit einer größeren Zahl identifizierter Lymphknoten errechnen (0 - 4 Lymphknoten, 5 - 10 Lymphknoten, $>10$ Lymphknoten). Dieser Effekt zeigt sich auch in jedem Tumorstadium. In der multivariaten Analyse erwies sich die Zahl untersuchter Lymphknoten als unabhängiger prognostischer Faktor. Eine Analyse der SEER-Datenbank [764] korreliert die Zahl der untersuchten bzw. entfernten Lymphknoten mit dem Langzeitüberleben. In der multivariaten Analyse zeigte sich eine Reduktion der Krebsmortalität um 20,6\% wenn mehr als 15 
Lymphknoten untersucht wurden, verglichen mit Patienten bei denen nur 1 - 7 Lymphknoten untersucht wurden. Dieses Ergebnis war unabhängig vom Tumorstadium und sonstigen Patienten- oder Tumorcharakteristika. Auch wenn die Studienlage hinsichtlich der genauen Anzahl der zu untersuchenden Lymphknoten nicht einheitlich ist, sollten nach Meinung von Experten 12 Lymphknoten als Mindestmaß gefordert werden, auch wenn diese Zahl nicht immer flächendeckend erreicht werden kann. In diesem Zusammenhang sei auf die anstehende Überarbeitung des TK „Chirurgie“ verwiesen.

In einer Studie, in denen Patienten mit Stadium-II-Tumoren und Hochrisikocharakteristika eine kleine Untergruppe darstellten, konnte kein Benefit einer adjuvanten Chemotherapie gegenüber der alleinigen operativen Therapie gezeigt werden [743]. Im Gegensatz dazu stellten in der MOSAIC-Studie Patienten mit Stadium-II-Tumoren mit einem T4 Tumor, einer Tumorperforation, einem Ileus, einem schlecht differenzierten Tumor, einer Blutgefäßinvasion und/oder weniger als 10 untersuchten Lymphknoten eine Hochrisikopopulation dar, bei der die postoperative adjuvante FOLFOX4 Chemotherapie im Vergleich zu einer 5FU/FS Chemotherapie zu einer tendenziellen Verbesserung des krankheitsfreien Überlebens um 7,2\% Prozentpunkte führte (HR 0,74; $95 \%$ CI: 0,52-1,06). Es konnte allerdings - möglicherweise bedingt durch die zu geringe Patientenzahl - keine signifikante Verbesserung des Gesamtüberlebens erreicht werden [752]*.

\subsection{Empfehlung (2008)}

Zusätzliche Parameter (z. B. CEA-Spiegel, Differenzierungsgrad, $18 q$ Verlust, isolierte Tumorzellen in Lymphknoten oder im Knochenmark, Mikrosatelliten-Status, DNA-Ploidie und TS/p53 Expression, Lymph- und Blutgefäßinvasion) sollen momentan nicht zur Indikationsstellung für eine adjuvante Chemotherapie benutzt werden. Empfehlungsgrad A, Level of Evidence 4, starker Konsens

\section{Hintergrund}

Für eine Reihe von Parametern konnte in einigen, aber nicht in allen Studien eine prognostische Bedeutung für das kolorektale Karzinom gezeigt werden. Es liegen jedoch keine prospektiven Studien zum Nutzen einer adjuvanten Chemotherapie bei Vorhandensein einzelner oder mehrerer dieser Faktoren vor. Der Differenzierungsgrad wird in manchen Arbeiten sowohl im Stadium III [670, 765] als auch im Stadium II und III [745] als ein unabhängiger prognostischer Faktor gewertet. Hingegen zeigt eine Analyse von Hermanek, dass der Differenzierungsgrad nur in einer Untergruppe des Stadiums III (jedes T N2 M0) eine zusätzliche prognostische Bedeutung besitzt [766].

Der 18q-Alleelverlust besitzt in mehreren Studien auch im Stadium II unabhängige prognostische Bedeutung [767 - 771].

Eine Studie bei Patienten mit einem kolorektalem Karzinom im Stadium II $(\mathrm{n}=70)$ kommt jedoch zu dem Schluss, dass der 18qVerlust keine prognostische Bedeutung besitzt [772]. Ferner ist unklar, ob Tumoren mit 18q-Verlust möglicherweise schlechter auf Chemotherapie ansprechen.

Mikrosatelliteninstabilität (MSI) kann in 10-15\% der sporadischen kolorektalen Karzinome nachgewiesen werden. Eine Mikrosatelliteninstabilität wird verursacht durch Defekte des DNA-Mismatch-Repair(MMR)-Systems durch Inaktivierung der MLH-1-, MSH-2-, MSH-6- und PMS-2-Gene. Die Ergebnisse einer Untersuchung von 718 Patienten aus Italien deuten darauf hin, dass Patienten mit einem MMR-Protein-negativen Tumor eine bessere Langzeitprognose aufweisen als Patienten mit einem MMR-Protein-positiven Karzinom. Dieser positive prognostische
Effekt war sowohl im Stadium II als auch im Stadium III vorhanden. Bei Patienten mit MMR-Protein-positiven Tumoren bewirkt die adjuvante Chemotherapie eine Prognoseverbesserung [773]. In einer Arbeit von Sinicrope waren Mikrosatelliteninstabilität und DNA-Diploidie ebenfalls mit einer besseren Prognose assoziiert [774].

Eine aktuell publizierte Metaanalyse zeigte eine klare negative prognostische Bedeutung der DNA Aneuploidie. Patienten mit einem aneuploiden kolorektalen Karzinom hatten fünf Jahre nach Operation eine deutlich höhere Sterblichkeitsrate als solche mit diploiden Tumoren. Dies galt für alle untersuchten Subgruppen, v.a. auch für das Stadium II [775].

Bei 570 Patienten im Stadium II (55\%) und Stadium III (45\%) aus der gemeinsamen IMPACT-Analyse führte die adjuvante Chemotherapie zu einer Verbesserung des Überlebens, bei hoher Mikrosatelliteninstabilität führte die adjuvante Chemotherapie eher zu einer Verschlechterung des Überlebens [776]. Eine Untersuchung von 876 Patienten mit einem Stadium-III-Tumor konnte zeigen, dass in der Gruppe ohne adjuvante Chemotherapie der Mikrosatellitenstatus keine prognostische Bedeutung besaß (5-JahresÜberlebensrate: 43 gegen $36 \%$ ), während in der chemotherapeutisch behandelten Gruppe die Patienten mit MSI positiven Tumoren ein signifikant besseres Überleben aufwiesen [777]. Keine dieser Arbeiten war bezüglich der Überprüfung der prognostischen und prädiktiven Parameter prospektiv angelegt.

\subsubsection{Chemotherapieprotokolle}

8.1.6.1. Stadium III

Oxaliplatin in Kombination mit 5-FU/Folinsäure (FS)

\subsection{Empfehlung (2008)}

Für die adjuvante Chemotherapie des Kolonkarzinoms im Stadium III soll eine Oxaliplatin-haltige Therapie eingesetzt werden. Empfehlungsgrad A, Level of Evidence 1, starker Konsens

\section{- FOLFOX (LV5FU2 + Oxaliplatin):}

z.B. FOLFOX4: Folinsäure (FS) $\left(200 \mathrm{mg} / \mathrm{m}^{2}\right.$ als 2-h-Infusion, Tag 1 und 2) plus 5-FU ( $400 \mathrm{mg} / \mathrm{m}^{2}$ als Bolus, danach $600 \mathrm{mg} /$ $\mathrm{m}^{2}$ als 22-h-Infusion; Tag 1 und 2) in Kombination mit Oxaliplatin $\left(85 \mathrm{mg} / \mathrm{m}^{2}\right.$ als 2-h-Infusion; Tag 1$)$, Wiederholung Tag 15. 1 Zyklus umfasst 2 Wochen, insgesamt 12 Zyklen.

\section{Hintergrund}

Durch die Kombination aus 5-FU und Folinsäure konnte in mehreren randomisierten Studien eine signifikanten Senkung der Rezidivrate sowie des Gesamtüberlebens gezeigt werden [742, 743, 746].

Die MOSAIC-Studie (2246 Patienten) verglich eine adjuvante Chemotherapie bestehend aus 5-FU/FS (LV5FU2) mit dem FOLFOX4 Schema (LV5FU2 + Oxaliplatin $85 \mathrm{mg} / \mathrm{m}^{2}$ ) alle 2 Wochen über 12 Zyklen. In dem Gesamtkollektiv führte die FOLFOX4 Chemotherapie zu einer signifikanten Verbesserung des krankheitsfreien Überlebens gegenüber der LV5FU2-Chemotherapie (73,3 vs. 67,4\%, p=0,003) [752, 778 $]^{*}$. Betrachtet man nur das Stadium III, ergibt sich für die FOLFOX4-Chemotherapie ein Unterschied im krankheitsfreien Überleben um 7,5 Prozentpunkte (HR 0,78; 95\% CI: 0,65-0,93; p =0,005). Auch das Gesamtüberleben wird im Stadium III durch die FOLFOX4 Chemotherapie signifikant um 4,4 Prozentpunkte verbessert $(p=0,029)$. 4 Jahre nach der Therapie lag die Rate der peripher-sensorischen Neuropathie bei $12 \%$ (Grad I), 2,8\% (Grad II) und 0,7\% (Grad III) [752, 778]. In die NSABP-Studie C-07 wurden 2407 Patienten im Stadium II $(28,6 \%)$ oder im Stadium III eingeschlossen und erhielten entweder das Roswell-Park-Schema mit wöchentlicher 5-FU/FS-Bolus- 
gabe (3 Zyklen à jeweils 8 Wochen) oder das gleiche 5-FU/FSSchema mit Oxaliplatin $85 \mathrm{mg} / \mathrm{m}^{2}$ in Woche 1,3 und 5 im achtwöchentlichen Rhythmus (FLOX-Schema). In der FLOX-Gruppe wurden $20 \%$ weniger Rezidive beobachtet ( $p<0,04$ ). Das krankheitsfreie Überleben nach 4 Jahren betrug $73,2 \%$ für die FLOXGruppe und 67,0\% für die 5-FU/FS-Behandelten [779]. Bei der Auswahl ist das unterschiedliche Nebenwirkungsprofil der einzelnen Protokolle zu berücksichtigen. Aufgrund der höheren kumulativen Oxaliplatindosis in der MOSAIC-Studie liegt die Rate der beobachteten Grad-3/4-Neuropathien in der NSABP-Studie geringfügig niedriger (12,4 vs. $8,4 \%$ ). In dem Bolus-FLOX-Protokoll wurden jedoch häufiger als in dem infusionalen FOLFOX4Protokoll Grad-3/4-Diarrhöen beobachtet (38 vs. 10,8\%). 5 Patienten $(0,4 \%)$ in der NSABP-Studie starben innerhalb der ersten 60 Tage nach Beginn der Chemotherapie aufgrund einer chemotherapieinduzierten Enteropathie [779]. Die Toxizität des FLOXProtokolls ist im Vergleich mit dem FOLFOX4-Protokoll bei vergleichbarer Wirksamkeit nicht tolerabel. Daher sollte das FLOXProtokoll in der adjuvanten Situation keine Anwendung finden. International wird derzeit dem modifizierten FOLFOX6 Schema, das nach einem 5-FU-Bolus an Tag 1 eine 46 stündige Dauerinfusion von 5-FU vorsieht, der Vorzug gegeben; dieses Schema ist der Kontrollarm internationaler Studien. Dies erspart dem Patienten den 5-FU-Bolus und den Pumpenwechsel an Tag 2 der Therapie (Dosierung: Oxaliplatin $85 \mathrm{mg} / \mathrm{m}^{2}$ i.v., Folinsäure $400 \mathrm{mg} / \mathrm{m}^{2}+5$-FU $400 \mathrm{mg} / \mathrm{m}^{2}$ Bolus, danach $2400 \mathrm{mg} / \mathrm{m}^{2}$ kontinuierlich i.v. über $46 \mathrm{~h}$ alle 2 Wochen). Zur Kombination von Capecitabin und Oxaliplatin im Vergleich zu verschiedenen Bolusregimen von 5-FU/FS liegen derzeit nur Toxizitätsdaten vor [780], Effektivitätsergebnisse werden für das Jahr 2008 erwartet. Eine adjuvante Therapie mit einem irinotecanhaltigen Protokoll ist auf Basis der vorliegenden Daten aus Phase-III-Studien nicht zu empfehlen [781 - 783].

\section{Monotherapie mit Fluoropyrimidinen}

\subsection{Empfehlung (2008)}

Bei Kontraindikationen gegen Oxaliplatin-haltige Regime soll eine Monotherapie mit Fluoropyrimidinen durchgeführt werden. Dabei werden orale Fluoropyrimidine den infusionalen Schemata vorgezogen. Bolusregime sollen wegen der höheren Toxizität nicht mehr verwendet werden.

Empfehlungsgrad A, Level of Evidence 1, starker Konsens

\section{- Orale 5-FU Prodrug:}

z. B. Capecitabin $2 \times 1250 \mathrm{mg} / \mathrm{m}^{2}$ Körperoberfläche p. o. Tag 1 14 ; alle 3 Wochen für 8 Zyklen

\section{Hintergrund}

1987 Patienten mit einen Stadium-III-Kolonkarzinom wurden entweder in das Mayo-Clinic-Schema (983 Patienten) randomisiert oder erhielten Capecitabin als Monotherapie (1004 Patienten) jeweils über eine Zeit von 24 Wochen (X-ACT Studie). Das primäre Studienziel mit dem Nachweis, dass Capecitabin im krankheitsfreien Überleben mindestens gleichwertig zum Mayo-Schema ist, wurde erreicht. Die Analyse zeigte einen Trend zu Gunsten eines überlegenen krankheitsfreien Überlebens mit Capecitabin (HR 0,87; 95\% CI: 0,75-1,00; p=0,05). Auch das Gesamtüberleben unterschied sich nicht signifikant, mit einem Trend zur Überlegenheit von Capecitabin (81,3 vs. 75,6\%; $\mathrm{p}=0,05)$ [784].

Auch wenn in einer randomisierten Studie UFT + Folinsäure versus 5-FU/FS [785] kein Unterschied im Gesamtüberleben und im krankheitsfreien Überleben bestand und in einer japanischen Metaanalyse von drei Studien sogar ein signifikanter Vorteil für
Gesamtüberleben und DFS erreicht werden konnte [786]wird UFT aktuell nicht empfohlen, da es keine Zulassung zur adjuvanten Chemotherapie des Kolonkarzinoms in Deutschland besitzt.

- Infusionales 5-FU/Folinsäure:

$\rightarrow$ LV5FU2

z. B. Folinsäure (FS) $(200 \mathrm{mg} / \mathrm{m} 2$ als 2-h-Infusion, Tag 1 und

2) plus 5 -FU $(400 \mathrm{mg} / \mathrm{m} 2$ als Bolus, danach $600 \mathrm{mg} / \mathrm{m} 2$ als

22-h-Infusion; Tag 1 und 2)

1 Zyklus umfasst 2 Wochen, insgesamt 12 Zyklen

-5-FU/Folinsäure-Schema

z. B. Folinsäure (FS) (500 mg/m2 als 1- bis 2-h-Infusion) plus 5 -FU $(2600 \mathrm{mg} / \mathrm{m} 2$ als $24 \mathrm{~h}$ Infusion) $1 \times$ pro Woche über 6 Wochen (Tag 1, 8, 15, 22, 29, 36). Erneuter Beginn der Therapie in Woche 9 (Tag 50). Insgesamt 2 Zyklen.

v venöse 5-FU-Dauerinfusion (PVI)

z.B. 5-FU als Dauerinfusion über insgesamt 12 Wochen (300 mg/m²/Tag)

\section{Hintergrund}

Im Vergleich zu den Bolusschemata zeigen mehrere Therapiestudien mit unterschiedlicher infusionaler Applikationsform keinen Unterschied zu der Bolusgabe von 5-FU/FS bez. des krankheitsfreien Überlebens und des Gesamtüberlebens. Das deutlich bessere Toxizitätsprofil spricht jedoch eindeutig für die infusionale Applikation [787, 788 $]^{*}[789,790]$. Ein Vergleich von 12 Wochen Therapie mit der „protracted venous infusion“ (PVI) von 5FU (300 mg/m² pro Tag) gegen ein 6-monatiges MAYO-Schema zeigte keinen signifikanten Unterschied im rezidivfreien Überleben (RFS) und im Gesamtüberleben bei geringerer Toxizität von PVI 5-FU [791]. Der Beginn der adjuvanten Chemotherapie innerhalb eines Zeitraums von 8 Wochen nach Operation zeigte einen signifikanten Überlebensvorteil [792]. Die optimale Dauer der Chemotherapie beträgt 6 Monate [789, 793, 794].

\subsubsection{Stadium II}

\subsection{Empfehlung (2008)}

Sollte bei Patienten mit Stadium-II-Tumoren eine adjuvante Chemotherapie durchgeführt werden, können Fluoropyrimidine als Monotherapie eingesetzt werden.

Empfehlungsgrad 0, Level of Evidence 1, starker Konsens

\section{Hintergrund}

siehe Kapitel 8.1.4 UICC-Stadium II.

\subsection{Perioperative Therapie beim Rektumkarzinom}

8.2.1. Indikationen zur perioperativen Radio- oder Radiochemotherapie

8.2.1.1. Stadium I

\subsection{Empfehlung (2008)}

Im Stadium I ist eine perioperative Therapie nicht indiziert. Empfehlungsgrad A, Level of Evidence 5, starker Konsens

\section{Hintergrund}

Rektumkarzinome im UICC-Stadium I (T1/2 N0) haben bei alleiniger radikaler Operation mit En-bloc-Lymphknotendissektion und totaler mesorektaler Exzision (TME) für Tumoren im unteren (bis $6 \mathrm{~cm}$ ab Anokutanlinie) und mittleren Rektumdrittel $(>6-12 \mathrm{~cm}$ ab Anokutanlinie) sowie partieller mesorektaler Exzision (PME) für Tumoren im oberen Rektumdrittel (>12-16 cm ab Anokutanlinie) niedrige Lokalrezidiv- und Fernmetastasenraten [795]. In den frühen amerikanischen Serien zum Stellenwert der neoadjuvanten Radiochemotherapie wurde dieses Tumorstadium 
daher ebenso ausgeschlossen wie in den modernen Studien zur neoadjuvanten Radiochemotherapie [796].

Die schwedischen und holländischen Studien zur präoperativen Kurzzeit-Vorbestrahlung mit $5 \times 5 \mathrm{~Gy}$ versus alleinige Operation haben das Tumorstadium I gleichwohl eingeschlossen. Die jüngere holländische Studie zeigte in einer Subgruppenanalyse keinen signifikanten Unterschied hinsichtlich der Lokalrezidivrate zwischen alleiniger TME und zusätzlicher Radiotherapie für Tumoren im UICC-Stadium I [795, 797]. In der älteren schwedischen Studie konnte ein signifikanter Vorteil der zusätzlichen Bestrahlung für das Stadium I gezeigt werden, allerdings war hier das Konzept der TME noch nicht umgesetzt worden [798].

Der Stellenwert einer Radio(chemo-)therapie vor oder nach lokaler Exzision eines T1-High-risk-Karzinoms (G3/4, L1, V1, Durchmesser größer $3 \mathrm{~cm}$, R1-Resektion) ist nicht gesichert [799]. Bei inkompletter Resektion (R1) oder Risiko-Konstellation (s. o.) soll eine radikale Tumorentfernung mit Lymphknotenentfernung innerhalb von 4 Wochen durchgeführt werden. Für Patienten mit tiefsitzenden T1-High-risk Karzinomen oder T2-N0-Tumoren im UICC-Stadium I, die eine Exstirpation ablehnen, ist die präoperative Radio(chemo-)therapie, gefolgt von lokaler Exzision, eine Option [800]. Dies ist jedoch ein nicht gesichertes Vorgehen.

\subsubsection{Stadium II/III}

\subsection{Empfehlung (2008)}

Im UICC-Stadium II und III ist die neoadjuvante Radio- oder Radiochemotherapie indiziert. Eine Sondersituation besteht bei cT1/2Karzinomen mit fraglichem Lymphknotenbefall; hier ist auch die primäre Operation (mit ggf. adjuvanter Radiochemotherapie bei $\mathrm{pN+}$ ) eine mögliche Behandlungsoption.

Empfehlungsgrad A, Level of Evidence 1b, starker Konsens

\section{Hintergrund}

Metaanalysen zeigen eine verbesserte Wirksamkeit der präoperativen im Vergleich zur postoperativen Bestrahlung [801, 802]. Eine frühe randomisierte Studie zur prä- versus postoperativen alleinigen Radiotherapie hatte eine signifikant reduzierte Lokalrezidivrate im präoperativen Arm gezeigt [803]. Die deutsche Studie zur adjuvanten und neoadjuvanten Radiochemotherapie (RCT) des Rektumkarzinoms im UICC-Stadium II und III (CAO/ARO/AIO-94) wies ebenfalls eine signifikante Reduzierung der Lokalrezidivrate im neoadjuvanten Arm auf [804]. Die Rate postoperativer Komplikationen war nach präoperativer RCT im Vergleich zur sofortigen Operation nicht erhöht, die akute und chronische Toxizität im präoperativen RCT-Arm insgesamt signifikant niedriger. Bei tiefsitzenden Tumoren, die der Chirurg vor Randomisation als exstirpationspflichtig eingeschätzt hatte, konnte die Rate sphinktererhaltender Operationsverfahren durch die Vorbehandlung im Vergleich zur sofortigen Operation verdoppelt werden. Als Problem jeder neoadjuvanten Therapie muss das potenzielle „Overstaging“ und die daraus resultierende „Überbehandlung“ von Patienten gewertet werden, bei denen fälschlicherweise ein wanddurchsetzender (T3) oder lymphknotenpositiver Tumor (N+) diagnostiziert wurde. Da insbesondere die Sensitivität und Spezifität der Beurteilung des Lymphknotenbefalls limitiert ist, wird bei T1/ 2-Tumoren mit bildgebend fraglichen $\mathrm{N}+$ als Option auch die primäre Operation als sinnvoll erachtet. Als weitere Selektionskriterien für eine primäre Operation werden von einigen Zentren und Studiengruppen - auch im Hinblick auf die mit der Radiotherapie assoziierten chronischen Nebenwirkungen $[805,806]$ - T3-Tumoren mit Infiltration ins mesorektale Fettgewebe unter $5 \mathrm{~mm}$ sowie Tumoren mit einem Abstand zur mesorektalen Resektionslinie von mehr als $1 \mathrm{~mm}$ (MRT-Diagnostik obligat) genannt [807, 808]. Diese Selektionskriterien müssen in Studien weiter geprüft werden.

\subsection{Empfehlung (2008)}

Der Stellenwert der Strahlentherapie des Rektumkarzinoms im oberen Drittel wird kontrovers diskutiert. Es kann eine adjuvante Therapie wie beim Kolonkarzinom oder eine perioperative Radio(chemo-)therapie wie beim Rektumkarzinom durchgeführt werden.

Empfehlungsgrad 0, Level of Evidence 3a, starker Konsens

\section{Hintergrund}

Folgende Argumente sprechen dafür, das obere Rektumdrittel (>12-16 cm ab Anokutanlinie, gemessen mit einem starren Rektoskop) wie ein Kolonkarzinom zu behandeln:

- Die Daten der amerikanischen Adjuvanzstudien, die die Radiochemotherapie bei der Behandlung des Rektumkarzinoms etabliert hatten, bezogen sich ausschließlich auf Rektumtumoren mit einem Abstand des distalen Tumorpols von der Anokutanlinie bis $12 \mathrm{~cm}$.

- In der holländischen TME-Studie konnte bei Tumoren im oberen Rektumdrittel (hier definiert als: $10-15 \mathrm{~cm}$ ab Anokutanlinie) keine signifikante Verbesserung der Lokalrezidivrate durch die zusätzliche Radiotherapie nachgewiesen werden [797].

Folgende Argumente sprechen dafür, das obere Rektumdrittel wie ein Rektumkarzinom zu behandeln:

- Bei der Analyse der holländischen TME-Studie handelt es sich um eine explorative Subgruppenanalyse. Daher wurde von den Autoren konsequenterweise nicht gefolgert, dass Patienten mit Tumoren im oberen Rektumdrittel keiner Radiotherapie bedürfen.

- In der bislang nur als Abstrakt publizierten britischen MRCCR07-Studie war der Vorteil der generellen präoperativen Kurzzeit-Radiotherapie versus selektiver postoperativer Radiochemotherapie nur bei befallenem zirkumferenziellen Resektionsrand für alle Rektumdrittel signifikant [809]*.

- Eine aktuelle Subgruppenanalyse der deutschen CAO/ARO/ AIO-Studie-94 zeigte keinen signifikanten Unterschied der Lokalrezidivraten zwischen Tumoren im mittleren und oberen Rektumdrittel.

- Im Gegensatz zu der holländischen TME-Studie werden in Deutschland Tumoren im oberen Rektumdrittel mittels partieller mesorektaler Exzision (PME) behandelt. Dieses Verfahren ist u.U. mit einer höheren Lokalrezidivrate verbunden. Die GAST-05-Studie wird unter Federführung von Prof. Becker/Dr. Liersch die Frage prüfen, ob Tumoren im oberen Rektumdrittel eine TME benötigen.

\subsection{Empfehlung (2008)}

In Situationen, in denen ein Downsizing angestrebt wird (T4-Tumore, nicht ausreichender Sicherheitsabstand im Dünnschicht-MRT zur mesorektalen Faszie - Abstand $1 \mathrm{~mm}$ oder weniger - oder erwünschter Sphinktererhalt bei Tumoren im unteren Drittel), soll der präoperativen Radiochemotherapie der Vorzug vor einer Kurzzeit-Radiotherapie gegeben werden. Bei cT3-Tumoren oder $\mathrm{CN}^{+-T u}$ moren, bei denen kein Downsizing angestrebt wird, kann die präoperative Therapie entweder als Radiochemotherapie oder als Kurzzeitbestrahlung erfolgen. Bei cT3-Tumoren oder $\mathrm{CN}^{+-}$-Tumoren, bei denen kein Downsizing angestrebt wird, kann die präoperative Therapie entweder als Radiochemotherapie oder als Kurzzeitbestrahlung erfolgen. 
Empfehlungsgrad A, Level of Evidence 3b, starker Konsens

\section{Hintergrund}

Zur präoperativen Radiotherapie stehen prinzipiell zwei Fraktionierungsschemata zur Verfügung: die Kurzzeitbestrahlung mit $25 \mathrm{~Gy}$ in Einzeldosen von $5 \mathrm{~Gy}$ an 5 aufeinander folgenden Tagen, unmittelbar gefolgt von der Operation, und die konventionell fraktionierte Bestrahlung bis zu einer Gesamtreferenzdosis von 45 bis 50,4 Gy in 25 - 28 Fraktionen, gefolgt von der Operation nach 4-6 Wochen. Eine randomisierte polnische Studie hatte nach neoadjuvanter konventionell fraktionierter Radiochemotherapie ein signifikant überlegenes Ergebnis hinsichtlich Downsizing und Downstaging sowie eine signifikant niedrigere Rate an R1-Resektionen im Vergleich zur Kurzzeitbestrahlung ergeben [810]. Allerdings waren die Rate an sphinktererhaltenden Operationsverfahren (primärer Endpunkt) sowie die lokale Kontrolle (sekundärer Endpunkt) in beiden Armen nicht signifikant unterschiedlich [811]. Zur Maximierung der Tumorschrumpfung vor Operation sollte bei den oben genannten Indikationen der konventionell fraktionierten Radiochemotherapie der Vorzug vor der Kurzzeitbestrahlung gegeben werden. Bei letzterer tritt durch die kurze Behandlungszeit und die sich unmittelbar anschließende Operation nämlich keine relevante Tumorschrumpfung ein [812].

\subsection{Empfehlung (2008)}

Die neoadjuvante Radiochemotherapie soll eine 5-FluorouracilMonochemotherapie mit oder ohne Folinsäure beinhalten. Empfehlungsgrad A, Level of Evidence 1b, starker Konsens

\section{Hintergrund}

Der Stellenwert der Kombination der konventionell fraktionierten präoperativen RT mit einer simultanen 5-FU/Folinsäure-Chemotherapie wurde in der EORTC 22921 sowie der FFCD 9203Studie untersucht (in der EORTC-Studie zusätzlich die Bedeutung der adjuvanten Chemotherapie [813, 814]). Wesentliches Ergebnis beider Studien ist die signifikante Reduzierung der Lokalrezidivrate durch die präoperative Radiochemotherapie im Vergleich zur alleinigen Radiotherapie. In der deutschen CAO/ARO/AIO-94Studie wurde 5-FU in der 1. und 5. Bestrahlungswoche in einer Dosierung von $1000 \mathrm{mg} / \mathrm{m}^{2} /$ Tag als 120 -stündige Dauerinfusion appliziert. In der EORTC 22 921- und FFCD 9203-Studie erhielten die Patienten in der 1. und 5. Bestrahlungswoche 5-FU in einer Dosierung von $350 \mathrm{mg} / \mathrm{m}^{2} /$ Tag und Folinsäure in einer Dosierung von $20 \mathrm{mg} / \mathrm{m}^{2} /$ Tag über jeweils 5 Tage.

Die neoadjuvante Radiochemotherapie mit neuen Substanzen und Kombinationen (Capecitabin, Oxaliplatin, Irinotecan) zeigte in einer Vielzahl von Phase-II-Studien Komplettremissions-Raten bis zu 30\% [815]. Der Stellenwert dieser Kombinationsbehandlungen wird derzeit in Phase-III-Studien geprüft.

\subsubsection{Adjuvante Therapie}

8.2.2.1. Adjuvante Therapie bei primärer Operation (ohne neoadjuvante Therapie)

\subsection{Empfehlung (2008)}

Im Stadium I ist nach R0-Resektion eine adjuvante Therapie nicht indiziert.

Empfehlungsgrad A, Level of Evidence 1b, starker Konsens

\section{Hintergrund}

In allen randomisierten Studien zur adjuvanten Therapie waren Patienten mit Stadium UICC I wegen insgesamt niedriger Lokalrezidiv- und Fernmetastasenraten ausgeschlossen worden.

\subsection{Empfehlung (2008)}

Bei Patienten im UICC-Stadium II und III, die keine neoadjuvante Radiochemotherapie oder Kurzzeit-Radiotherapie erhalten haben, soll eine adjuvante Radiochemotherapie erfolgen.

Empfehlungsgrad A, Level of Evidence 1b, starker Konsens

\section{Hintergrund}

Durch Hinzunahme der Chemotherapie zu einer postoperativen Bestrahlung konnten sowohl die Lokalrezidivrate gesenkt als auch das Gesamtüberleben im Vergleich zur alleinigen (konventionellen) Operation verbessert werden [816, 817]. Daten zum Nutzen einer adjuvanten Radiochemotherapie nach pathologisch bestätigter adäquater Mesorektumexzision und einem Abstand des Tumors zum zirkumferenziellen Resektionsrand von mehr als $1 \mathrm{~mm}$ liegen bislang nicht vor. Die Lokalrezidivraten werden hier auch ohne zusätzliche adjuvante Therapie mit global unter $10 \%$ angegeben, können aber für Subgruppen, z. B. Tumoren im unteren Rektumdrittel, auch höher liegen. Patienten mit Tumoren im UICC-Stadium II und III sollten in randomisierte Studien eingebracht werden. Dabei wäre zu klären, ob es nach qualitätsgesicherter Chirurgie Subgruppen von Patienten gibt (z.B. pT3N0-Tumoren mit geringer Infiltration ins perirektale Fettgewebe oder pT1/2 N+-Tumoren), die ein dem UICC-Stadium I vergleichbares Rezidivrisiko haben und daher von einer adjuvanten Radio- und Chemotherapie nicht profitieren $[818,819]$. Zur Frage der Therapie von Tumoren im oberen Rektumdrittel (s. 8.2.1.2).

In der bislang nur als Abstrakt publizierten britischen MRCCR07-Studie hat sich gezeigt, dass ein risikoadaptiertes Vorgehen (postoperative Radiochemotherapie nur bei Patienten mit positiven zirkumferenziellen Resektionsrand nach TME) im Vergleich zu einer generellen präoperativen Radiotherapie mit $5 \times 5$ Gy für alle Rektumkarzinome hinsichtlich der lokalen Kontrolle und dem krankheitsfreien Überleben signifikant unterlegen ist [809].

\subsection{Empfehlung (2008)}

Nach R1-Resektion oder intraoperativem Tumoreinriss sollte postoperativ radiochemotherapiert werden, falls keine neoadjuvante Radio(chemo)therapie vorangegangen ist.

Empfehlungsgrad B, Level of Evidence 4, starker Konsens

\section{Hintergrund}

R1-Resektionen und intraoperativer Tumoreinriss sind mit einem hohen Lokalrezidivrisiko assoziiert und rechtfertigen die postoperative RCT. Eine ungeplante Subgruppenanalyse der holländischen TME-Studie hatte keine signifikante Verbesserung der Lokalrezidivraten durch eine postoperative alleinige Radiotherapie bis 50,4 Gy gezeigt [820].

\subsection{Empfehlung (2008)}

Die adjuvante Therapie sollte 4-6 Wochen nach der Operation beginnen.

Empfehlungsgrad B, Level of Evidence 3a, starker Konsens

\subsection{Empfehlung (2008)}

Die Strahlentherapie kann zeitgleich zum 1. und 2. Chemotherapiezyklus oder zum 3. und 4. Zyklus erfolgen.

Empfehlungsgrad 0, Level of Evidence 2a, starker Konsens 


\subsection{Empfehlung (2008)}

Die Strahlentherapie soll mit einer 5-FU-Monochemotherapie kombiniert werden.

Empfehlungsgrad A, Level of Evidence 1b, starker Konsens

\section{Hintergrund zu den letzten 3 Empfehlungen}

Nach dem „NCI-Schema“ beginnt die adjuvante Therapie 4 - 8 Wochen nach Operation mit der Applikation von 2 Chemotherapiekursen 5-FU in einer Dosierung von $500 \mathrm{mg} / \mathrm{m}^{2} \mathrm{KO} / \mathrm{Tag}$ als Bolusapplikation an 5 aufeinanderfolgenden Tagen (Tag 1 bis 5 und 36 bis 40). Die Radiotherapie beginnt am Tag 63. Das pelvine Lymphabflussgebiet erhält bei einer Einzeldosis von 1,8 Gy und fünfmal wöchentlicher Bestrahlung eine Gesamtdosis von $45 \mathrm{~Gy}$, anschließend erfolgt eine kleinvolumige Dosisaufsättigung im Gebiet des größten Lokalrezidivrisikos bis 50,4 Gy. In der ersten und fünften Bestrahlungswoche erhalten die Patienten eine simultane 5-FU-Chemotherapie in gleicher Dosierung und Applikationsform wie bei den initialen 2 Kursen, jedoch nur über 3 Tage. Nach Beendigung der Radiotherapie folgen 2 weitere Kurse Chemotherapie (Tag 134-138 und 169-173), allerdings mit einer reduzierten Dosis von $450 \mathrm{mg}$ 5-FU/m² KO/Tag über fünf Tage [514].

Nach den von O'Connell et al. veröffentlichten Studienergebnissen kann während der gesamten Bestrahlung statt der 5-FU-Bolusgabe auch eine niedrigdosierte 5-FU-Dauerinfusion in einer Dosierung von $225 \mathrm{mg} / \mathrm{m}^{2} \mathrm{KO} /$ Tag verabreicht werden [821]. Die Intergroup0144-Studie konnte allerdings die Überlegenheit von 5-FU-Dauerinfusionsprogrammen im Vergleich zu biochemisch (Folinsäure/ Levamisol) modulierten 5-FU-Bolusgaben nicht bestätigten [822]. Die Modulation von 5-FU-Bolusgaben mit Leukovorin und/oder Levamisol erwies sich in einer vierarmigen amerikanischen Intergroup-Studie (0114) der alleinigen 5-FU-Bolusgabe als nicht überlegen [823].

Eine weitere mögliche Modifikation des NCI-Schemas betrifft den zeitlichen Abstand zwischen Operation und Radiotherapie. Tumor- und strahlenbiologische Gründe sprechen für ein enges zeitliches Intervall zur Operation. Erste Analysen einer koreanischen Studie lieferten Hinweise, dass durch den frühzeitigen Beginn der Radiotherapie zeitgleich mit den ersten beiden postoperativen Chemotherapiekursen ein signifikant besseres krankheitsfreies Überleben erreicht werden kann [824]. Dies hat sich jedoch bei längerer Nachbeobachtungszeit dieser Studie nicht bestätigt [825]*. Der postoperative Arm der deutschen Studie CAO/ARO/AIO-94 kann als Alternative zum NCI-Schema empfohlen werden (RCT-Beginn 4 Wochen nach Operation, $1000 \mathrm{mg} / \mathrm{m}^{2}$ / Tag 5-FU als 120-h-Dauerinfusion in der ersten und fünften Bestrahlungswoche, 4 Kurse adjuvante Chemotherapie 5-FU-Bolus in einer Dosierung von $500 \mathrm{mg} / \mathrm{m}^{2} /$ Tag über 5 Tage, 3 Wochen Pause).

\subsection{Empfehlung (2008)}

Standard für die adjuvante Therapie des Rektumkarzinoms ist die kombinierte Radiochemotherapie. Eine Indikation für eine alleinige (adjuvante) Chemo- oder Radiotherapie beim Rektumkarzinom besteht nicht. Eine Ausnahme stellt nur die Kontraindikation gegen eine der beiden Therapieformen dar.

Empfehlungsgrad A, Level of Evidence 1a, starker Konsens

\section{Hintergrund}

Die alleinige postoperative Radiotherapie reduziert die Lokalrezidivrate, hat aber im Gegensatz zur Kombination aus Radiound Chemotherapie keinen Einfluss auf das Gesamtüberleben gezeigt [826]. Kontraindikationen für eine Radiotherapie stellt eine Vorbestrahlung im Becken wie z.B. im Rahmen der Behandlung eines Prostata- oder eines Zervixkarzinoms dar. Durch eine alleinige Chemotherapie wird das Rezidivrisiko zwar gesenkt, die Kombination mit einer Radiotherapie war der alleinigen Chemotherapie jedoch überlegen [514]. In einer kürzlich publizierten japanischen Phase-III-Studie konnte allerdings durch eine postoperative alleinige Chemotherapie mit Uracil-Tegafur nach TME und selektiver lateraler Lymphknotendissektion ein Überlebensvorteil für Patienten im UICC-Stadium III nachgewiesen werden [827].

8.2.2.2. Adjuvante Therapie nach neoadjuvanter Radiotherapie oder Radiochemotherapie

\subsection{Empfehlung (2008)}

Nach neoadjuvanter Radiochemotherapie ist eine adjuvante Chemotherapie unabhängig vom postoperativen Tumorstadium (also auch bei kompletter Remission oder UICC-Stadium I und II) indiziert.

Empfehlungsgrad A, Level of Evidence 1b, starker Konsens

\section{Hintergrund}

Grundlage dieser Empfehlung ist, dass die adjuvante Chemotherapie obligater Bestandteil der CAO/ARO/AIO-94-Studie sowie der FFCD 9203-Studie nach erfolgter präoperativer Radiochemotherapie war. Die EORTC (22 921) randomisierte in einer vierarmigen Studie und einem „two-by-two factorial design“ zwischen einer postoperativen Chemotherapie und keiner postoperativen Chemotherapie nach präoperativer Radiotherapie oder Radiochemotherapie. Die postoperative Chemotherapie führte dabei zwar nicht zu einer statistisch signifikanten Überlebensverbesserung. Der Überlebensbenefit betrug jedoch $6 \%$ absolut für das progressionsfreie und $4 \%$ für das Gesamtüberleben und wurde mit einer vergleichsweise wenig toxischen Therapie erzielt [804]. Subgruppenanalysen zeigen, dass die adjuvante Chemotherapie insbesondere für diejenige Patientengruppe einen signifikanten Überlebensvorteil ergab, die nach präoperativer Therapie eine histopathologische ypT0/1/2-Kategorie aufwiesen [804]. In den Studien zur präoperativen Kurzzeit-Radiotherapie mit $5 \times 5 \mathrm{~Gy}$ wurde generell keine adjuvante Chemotherapie appliziert. Eine aktuelle holländische Phase-III-Studie randomisiert derzeit nach $5 \times 5 \mathrm{~Gy}$ und Operation zwischen einer adjuvanten Chemotherapie mit Capecitabin und Beobachtung $[813,828]$.

\subsection{Empfehlung (2008)}

Die adjuvante Chemotherapie sollte entweder als 5-FU Monotherapie oder als Kombination aus 5-FU/Folinsäure durchgeführt werden.

Empfehlungsgrad B, Level of Evidence 1b, starker Konsens

\section{Hintergrund}

In der CAO/ARO/AIO-94-Studie wurden 4 Zyklen adjuvanter Chemotherapie mit 5-FU in einer Dosierung von $500 \mathrm{mg} / \mathrm{m}^{2}$ als i. v. Bolus über 5 Tage alle 4 Wochen verabreicht. In der EORTC 22 921- und FFCD 9203-Studie erhielten die Patienten 4 Zyklen einer adjuvanten Chemotherapie mit 5-FU in einer Dosierung von $350 \mathrm{mg} / \mathrm{m}^{2} /$ Tag und Folinsäure in einer Dosierung von $20 \mathrm{mg} / \mathrm{m}^{2} /$ Tag über jeweils 5 Tage alle 4 Wochen. 


\section{Therapeutisches Vorgehen bei Metastasierung und in der palliativen Situation \\ $\nabla$}

Der folgende Teil der S3-Leitlinie enthält 2007/2008 aktualisierte Empfehlungen zur Tumortherapie beim metastasierten Kolorektalkarzinom, die vor allem Erkenntnisse aus Studien der Jahre 2003 - 2007 Jahre widerspiegeln. Auf die primär resektablen Metastasen wird ebenso eingegangen wie auf die besondere Situation einer sekundären Resektabilität in einem primär palliativen Therapiekonzept. Der Verfügbarkeit neuer biologischer Substanzen wird in einer kommentierten Auflistung möglicher Kombinationen in Abhängigkeit von Therapieziel und individueller Patientensituation Rechnung getragen. Die Einteilung der Patienten in Subgruppen soll die Entscheidungsfindung erleichtern.

\section{Definition von Subgruppen nach klinischen Situationen/ Therapiezielen}

1. Patienten mit primär resektablen Leber- und/oder Lungenmetastasen

2. Patienten mit einer Indikation für eine intensivierte systemische Therapie:

1. Patienten mit Leber- und/oder Lungenmetastasen, potenziell resektabel nach Ansprechen auf neoadjuvante Therapie und klinisch operable Patienten

2. Patienten mit tumorbedingten Symptomen, Organkomplikationen oder raschem Progress

3. Patienten mit der Möglichkeit für eine weniger intensive Therapie

1. Patienten mit multiplen Metastasen ohne Option für Resektion nach Metastasenrückbildung, ohne tumorbezogene Symptome oder Organkomplikationen und/oder schwerer Komorbidität

Sowohl bei synchronen als auch bei metachronen Metastasen der Leber und/oder Lunge stellt deren vollständige operative Entfernung für einen Teil der Patienten eine Heilungschance dar. Die Entscheidung über das therapeutische Vorgehen bei Nachweis einer pulmonalen und/oder hepatischen Metastasierung beginnt daher mit der Beurteilung der Resektabilität (Zitat: NCCN 2007). Bei R0-resektablen Metastasen sollte primär die Operation angestrebt werden (s. 9.1). Diejenigen Patienten, für die primär keine chirurgische Interventionsmöglichkeit besteht, sollten eine systemische Chemotherapie erhalten. Die Wahl des Chemotherapieregimes hängt dabei (entscheidend) vom Therapieziel ab. Die therapeutische Strategie bei Metastasierung und in der Palliativsituation sollte z. B. im Rahmen einer Tumorkonferenz interdisziplinär festgelegt werden. Die Patienten sind ihren individuellen Erfordernissen entsprechend ausführlich über die Therapieoptionen aufzuklären und in die Entscheidung miteinzubeziehen. Neben der Tumortherapie, die im Folgenden dargestellt wird, sind die Sicherstellung einer adäquaten Schmerztherapie und Ernährung, eine bedarfsgerechte psychosoziale und psychoonkologische Versorgung sowie supportive Therapiemaßnahmen integrale Bestandteile des palliativen Therapiekonzepts (s. themenspezifische Leitlinien unter www.awmf-leitlinien.de). (Verfügbare Quellen, Stand 08/07: Leitlinien der Deutschen Gesellschaft für Ernährungsmedizin/ ESPEN Guidelines on Enteral Nutrition (www.awmf-leitlinien. de, Registernr. 073/006e und 073/005e); LL Tumorschmerz von DIVS und DKG angemeldet und in Entwicklung, Fertigstellung 2008 geplant). Bezüglich der Therapieziele in der Palliativsituation wird dabei die krankheits- und therapiebezogene Lebensqualität als sehr gut messbarer Parameter immer häufiger als ein sekundärer Endpunkt von Studien erhoben. Dem Wunsch der Patienten nach Informationen über alle relevanten verfügbaren Maßnahmen (tumorspezifische, supportive, psychosoziale, psychoonkologische Therapieoptionen) und Hilfsangebote (z. B. Krebsberatungsstellen,

Selbsthilfegruppen) ist nachzukommen. Auch komplementäre/ unkonventionelle Behandlungsmethoden sollten offen mit den Patienten besprochen werden, auch um ungünstige Wechselwirkungen mit anderen Therapeutika zu vermeiden.

\subsection{Primär resektable Leber und/oder Lungen- metastasen \\ 9.1.1. Primär resektable Lungenmetastasen \\ 9.1. Empfehlung (2008) \\ Resektable Lungenmetastasen sollen reseziert werden. Empfehlungsgrad A, Level of Evidence 3a, starker Konsens}

\section{Hintergrund}

Die Indikation zur primären Resektion von Lungenmetastasen hängt von deren Anzahl und Lokalisation, dem Grad etwaiger vorhandener pulmonaler Vorschädigungen und dem zu erwartendem Residualvolumen nach Resektion ab. Voraussetzung ist, dass eine R0-Resektion grundsätzlich möglich erscheint. Die Resektionen sollen parenchymschonend sein, wobei eine ausreichende Radikalität gewährleistet sein sollte. Die Patienten sollten in spezialisierten Zentren mit entsprechend erfahrenen Operateuren vorgestellt werden [829]. In einem aktuell publizierten systematischen Review von Pfannschmidt et al. konnten in einer Mehrzahl der untersuchten Studien übereinstimmend nur die präoperativen CEA-Werte als unabhängiger prognostischer Faktor identifiziert werden, die Angaben zur prognostischen Relevanz der präoperativen Anzahl der Metastasen sind inkonsistent, zeigen aber einen Trend zu einem Überlebensvorteil bei singulärer Metastasierung ( $\bullet$ Tab.9). Weitere mögliche Einflussfaktoren wie das krankheitsfreie Intervall oder das initiale Tumorstadium haben sich hinsichtlich ihrer prognostischen Aussagekraft nicht bestätigt [830-833].

\subsubsection{Primär resektable Lebermetastasen}

Definition [834]: Resektable Lebermetastasen liegen vor, wenn

- eine nicht resektable extrahepatische Tumormanifestation ausgeschlossen ist

- weniger als $70 \%$ des Parenchyms befallen sind

- weniger als 3 Lebervenen und weniger als 7 Segmente betroffen sind

- keine Leberinsuffizienz oder Child B- oder C-Zirrhose vorhanden ist

- keine schwerwiegenden Begleiterkrankungen vorliegen

\subsection{Empfehlung (2008)}

Auf die Leber beschränkte R0-resektable Metastasen sollen reseziert werden.

Empfehlungsgrad A, Level of Evidence 3b, starker Konsens

\section{Hintergrund}

Das 5-Jahresüberleben nach Resektion kolorektaler Lebermetastasen liegt zwischen 25 und $40 \%$ [835 - 838] (० Tab. 10).

\subsection{Konsensbasierte Empfehlung (2008)}

Die Resektabilität von Metastasen soll durch einen in der Metastasenchirurgie erfahrenen Chirurgen beurteilt werden.

GCP, starker Konsens 


\begin{tabular}{|c|c|c|c|c|}
\hline Referenz & $\mathbf{n}$ & 5-Jahres-Überleben & $\begin{array}{l}\text { mittlere Nachbeobachtung } \\
\text { (Mo) }\end{array}$ & Evidenzlevel \\
\hline Lee 2007 & 59 & $50,3 \%$ & 34,7 & III \\
\hline Pfannschmidt 2003 & 167 & $32,4 \%$ & 58,6 & III \\
\hline Saito 2002 & 165 & $39,6 \%$ & 56,5 & III \\
\hline
\end{tabular}

Tab.9 5-Jahres-Gesamtüberleben nach R0-Resektion pulmonaler Metastasen (exemplarisch nach Pfannschmidt et al. [833]).

Tab. 10 Überleben nach Resektion kolorektaler Metastasen.

\begin{tabular}{|c|c|c|c|c|c|c|}
\hline Referenz & $\mathbf{n}$ & Operative Letalität & 5-Jahres-Überleben OP & $\begin{array}{l}\text { 5-Jahresüberleben } \\
\text { keine OP }\end{array}$ & $p=$ & Evidenzlevel \\
\hline Nordlinger $1996^{1}$ & 1568 & $2,3 \%$ & $28 \%$ & - & - & III \\
\hline Fong $1997^{2}$ & 456 & $2,8 \%$ & $38 \%$ & - & - & III \\
\hline Scheele $2001^{3}$ & 516 & $8,3 \%$ & $38 \%$ & - & - & III \\
\hline Kato $2003^{4}$ & $\begin{array}{l}763 \text { ( } 585 \text { OP vs. } \\
178 \text { keine OP) }\end{array}$ & n. a. & $39,2 \%$ & $3,4 \%$ & $<0,001$ & III \\
\hline
\end{tabular}

${ }^{1}$ Adjuvante Therapie bei $35 \%$ der Fälle.

${ }^{2}$ Adjuvante Therapie bei 128 Patienten.

343 Patienten mit unterschiedlichen Chemotherapieregimen vor Metastasenresektion, 26 Patienten adjuvant behandelt.

${ }^{4}$ Adjuvante Therapie bei $54,5 \%$ der Fälle ohne signifikanten Unterschied im Überleben (ÜL).

Tab. 11 Überleben nach Resektion kolorektaler Lebermetastasen (nach Fong et al. Annals of Surgery, 1999) [839].

\begin{tabular}{|l|l|}
\hline Score & 5-Jahres-Überleben \\
\hline 0 & $57 \%$ \\
\hline 1 & $57 \%$ \\
\hline 2 & $47 \%$ \\
\hline 3 & $16 \%$ \\
\hline 4 & $8 \%$ \\
\hline 5 & $0 \%$ \\
\hline
\end{tabular}

\section{Hintergrund}

Die Prognose kann präoperativ anhand einfach zu erfassender klinischer Kriterien nach dem sog. FONG-Score abgeschätzt werden ( Tab. 11) Dieser präoperative Prognosescore aus dem Memorial Sloan Kettering Cancer Center in New York wurde anhand einer großen Patientenzahl errechnet und ist international anerkannt.

Prognostisch ungünstige Kriterien [839]:

- Nodal positiver Primärtumor

- Krankheitsfreies Intervall $<12$ Monate

- Metastasengröße $>5 \mathrm{~cm}$

- Anzahl der Metastasen $>1$

- CEA präoperativ $>200 \mathrm{ng} / \mathrm{dl}$

Patienten, bei denen ein Score von bis zu maximal 2 Punkten vorliegt, haben eine gute Chance auf ein Langzeitüberleben nach primärer Leberresektion:

\subsubsection{Präoperative Bildgebung}

\subsection{Empfehlung (2013)}

Eine PET-CT kann bei Patienten mit resektablen Lebermetastasen eines kolorektalen Karzinoms durchgeführt werden.

Empfehlungsgrad 0, Level of Evidence 2b, Konsens

\subsection{Empfehlung (2013)}

Eine PET-CT sollte nicht innerhalb von 4 Wochen nach Gabe einer systemischen Chemotherapie oder Antikörpertherapie durchgeführt werden, da die Sensitivität deutlich reduziert ist. Empfehlungsgrad B, Level of Evidence 2b, starker Konsens

\section{Hintergrund}

Zur Frage des Nutzens einer PET/PET-CT bei der Rezidivdiagnostik und dem Rezidivstaging bei Patienten mit kolorektalem Karzinom war im August 2011 vom IQWIG (Institut für Qualität und Wirtschaftlichkeit im Gesundheitswesen)ein Vorbericht vorgelegt worden. Neben der Bewertung des Patienten relevanten Nutzens sollte eine systematische Bewertung der prognostischen und diagnostischen Güte der PET/PET-CT durchgeführt werden. Die Literaturrecherche umfasste den Zeitraum bis August 2009. Aktuellere Publikationen wurden im Text erwähnt. Den hier getroffenen Leitlinienempfehlungen liegen die Evidenzbewertungen des IQWIG Berichts zugrunde. Der Empfehlungsgrad wurde unter zusätzlicher Einbeziehung der klinischen Bewertung des Verfahrens erarbeitet. Ferner wurden in einer neueren Literaturrecherche von August 2009 - Dezember 2011 ein RCT in Abstraktform [548], ein systematisches Review [553], eine prospektive Fallkontrollstudie [549] und eine retrospektive Fallserie [551] identifiziert, die in die Bewertung ebenfalls mit eingegangen sind (Einzelheiten siehe Evidenzbericht). Die bisherige Empfehlung mit einem Grad B für die PET/PET-CT-Untersuchung vor einer Resektion von kolorektalen Lebermetastasen bei einem FONG-Score > 2 der letzten Leitlinienaktualisierung 2008 wurde geändert, da die Studie, die zu dieser Empfehlung führte, bis heute nicht als Vollmanuskript publiziert ist [840].

Zur Frage des Patienten-relevanten Nutzens der PET/PET-CT ist bislang eine RCT als Vollpublikation und 1 RCT in Abstraktform publiziert. Bei Patienten vor Resektion von Lebermetastasen eines KRK hat eine ergänzende PET/PET-CT keinen Einfluss auf das krankheitsfreie oder Gesamtüberleben des Patienten. Ob die ergänzende PET-CT Untersuchung überflüssige Laparotomien als klinisch relevanten Endpunkt vermeiden kann, ist nicht vollständig geklärt. Die hier konsentierte Empfehlung stützt sich vor allem auf die voll publizierte Studie von Ruers [547], die jedoch methodische Schwächen aufweist. In dieser Studie wurden 150 Patienten mit kolorektalen Lebermetastasen, die zur Resektion vorgesehen waren, in zwei Arme randomisiert, CT oder CT plus 18F-FDG PET. Das primäre Studienziel wurde in der Publikation angegeben als die Rate an überflüssigen Laparotomien, die durch die PET-Untersuchung eingespart werden kann. Dieser Endpunkt ist für den Patienten relevant. Die Studie fand keinen signifikanten Unterschied 
im Überleben im PET-Arm, jedoch eine signifikante Verringerung der Zahl „überflüssiger Laparotomien“ im PET-Arm. So war im Kontrollarm die Rate an überflüssigen Laparotomien $45 \%$, im PET-Arm 28\%. Dies entsprach einer Risikoreduktion von 38\% mit sehr großem Konfidenzintervall (95\% CI, 4-60\%, p=0,042). Die Autoren schlussfolgern, dass man mit einer zusätzlichen PET vor Lebermetastasenresektion einem von sechs Patienten die Laparotomie ersparen könnte. Die sekundären Endpunkte DFS und OS waren wie folgt: DFS: 35,5\% vs. 29,8\% (p-Wert=0,194); OS: $61,3 \%$ vs. $65,8 \%$ (p-Wert=0,378). Die Studie wurde von der Konsensuskonferenz im Evidenzlevel herabgestuft (s. auch IQWIG-Bericht, Herabstufung von Ib auf II), da der bei der Studienplanung genannte primäre Studienendpunkt von dem in der Publikation angegeben abwich (ursprünglicher Endpunkt: Rate der Patienten, die nach 9 Monaten krankheitsfrei sind).

Eine weitere, bislang auf dem ASCO-Jahresmeeting 2011 in Abstraktform vorgestellte multizentrische randomisierte Studie untersuchte ebenfalls diese Fragestellung [548]. Endpunkt dieser Studie war die Änderung im Patientenmanagement nach PET-Diagnostik (nicht durchgeführte OP wegen zusätzlicher Befunde oder Ausweitung der OP im Vergleich zur Intention ohne/vor der PET-Diagnostik) in einem 2:1 randomisierten Design bei Patienten mit KRK, die für eine Leberresektion bei Lebermetastasen geeignet erschienen. Es wurden 404 Patienten randomisiert (270 Patienten im PET/CT-Arm, 134 Patienten ohne PET). Man fand keinen Unterschied hinsichtlich der Managementänderung zwischen beiden Armen. Der Endpunkt wurde somit nicht erreicht. Allerdings hatten - soweit dies aus der Präsentation zu erheben war - ca. 70\% der Patienten vor der PET Diagnostik eine Chemotherapie erhalten, was die Sensitivität der Untersuchungstechnik deutlich reduziert (s. u.). Kritisch anzumerken gilt ferner, dass der Endpunkt „Änderung im Patientenmanagement“ im Gegensatz zu „Verringerung überflüssiger Operationen“ nicht als Patienten-relevant gilt.

Eine Reihe von Untersuchungen weisen darauf hin, dass sich die Sensitivität einer PET deutlich reduziert, falls es innerhalb von 4 Wochen nach einer Chemotherapie durchgeführt wird (Evidenzlevel IIa-III). Daher wird eine PET in diesem Zeitraum nicht empfohlen, da zu viele falsch negative Fälle auftreten. Diese Fragestellung hat der IQWIG Bericht nicht bewertet. In einer größeren Fallkontrollstudie, die die Sensitivität einer PET nach Chemotherapie nicht randomisiert prüfte, wurde 2010 publiziert [549]. Die Studie fand einen negativ prädiktiven Wert von nur 13,3\% und einen positiven prädiktiven Wert von $94 \%$ mit einer Spezifi- tät von 22,2\% bei einer Accuracy von $85 \%$, falls die PET innerhalb von vier Wochen nach Chemotherapieende durchgeführt wurde. Die Autoren schlussfolgern, dass eine diagnostische PET-Untersuchung kurz nach einer Chemotherapiegabe nicht sinnvoll sei. In einer retrospektiven Untersuchung aus Australien wurden PETErgebnisse von Patienten mit Lebermetastasen vor Leberresektion ausgewertet. Auch diese Studie war klein und heterogen. 21 Patienten wurden präoperativ systemisch therapiert, 53 nicht. Korrekte Ergebnisse mittels PET wurden für 29\% nach Chemotherapie und $53 \%$ in der Nicht-Chemotherapie-Gruppe erzielt. Unterschätzte Befunde ergaben sich zu 52 \% in der Chemotherapiegruppe, nur zu 34\% in der Nicht-Chemotherapie-Gruppe. Diese Studie gibt ebenfalls Hinweise darauf, dass eine PET-Untersuchung kurz nach einer Chemotherapie nicht sinnvoll ist. Eine weitere prospektive [550] und eine retrospektive Untersuchung [551] kamen zu ähnlichen Ergebnissen.

\subsubsection{Perioperative Therapie primär resektabler}

Lebermetastasen

\subsection{Neoadjuvante Therapie resektabler Lebermetastasen}

\subsection{Empfehlung (2008)}

Eine neoadjuvante systemische Therapie resektabler Lebermetastasen kann in begründeten Ausnahmefällen erwogen werden. Empfehlungsgrad 0, Level of Evidence 3, starker Konsens

\section{Hintergrund}

Eine neoadjuvante Therapie soll die Ergebnisse einer kurativ intendierten chirurgischen Intervention verbessern und ist damit an die realistische Option einer nachfolgenden R0-Resektion geknüpft. Zum Vergleich einer neoadjuvanten (präoperativen) oder kombinierten (perioperativen) vs. einer adjuvanten (postoperativen) Strategie hinsichtlich der Zielgröße R0-Resektabilität und Langzeitüberleben bei primär resektablen Lebermetastasen liegen keine prospektiven, randomisierten Studien vor. In einer aktuell publizierten, prospektiv randomisierten Phase-III-Studie der EORTC wurde der Stellenwert einer perioperativen Therapie bei primär als R0-resektabel eingestuften Lebermetastasen untersucht. Die Intention-to-treat-Analyse wies bez. des progressionsfreien Überlebens keinen signifikanten Vorteil für eine perioperative Therapie mit FOLFOX4 im Vergleich zu einer alleinigen Operation auf. Der Unterschied im 3 Jahres progressionsfreien Überleben lag bei $7,3 \%$ (28,1 bis $35,4 \%$, ) und verfehlte knapp das Signifikanzniveau (HR: 0,79; [0,62-1,02]; p=0,058) ( Tab. 12). Die „Per protocol“-

Tab. 12 Prospektive Studien zur neoadjuvanten Therapie bei Patienten mit resektablen Lebermetastasen.

\begin{tabular}{|c|c|c|c|c|c|c|}
\hline Referenz & $\mathbf{n}$ & Therapieregime & Ansprechrate (ORR) & Resektionsrate (R0) & Langzeitüberleben & Evidenzlevel \\
\hline Lorenz 2003 [843] ${ }^{1}$ & 42 & FOLFOX & $47,7 \%$ & $80,9 \%$ & - & IIb - III \\
\hline Wein $2003[844]^{2}$ & 20 & 5FU/FS/OX & $100 \%$ & $80 \%$ & $\begin{array}{l}\text { 2-Jhr. -tumorbezogenes } \\
\text { Überleben } \\
80 \%\end{array}$ & IV \\
\hline $\begin{array}{l}\text { Gruenberger } 2008 \\
\text { [845] }\end{array}$ & 56 & $\begin{array}{l}\text { XELOX } \\
+ \text { Bevacizumab }\end{array}$ & $73 \%$ & $93 \%$ & - & IIb \\
\hline $\begin{array}{l}\text { Nordlinger } 2008 \\
{[841]^{3}}\end{array}$ & & $\begin{array}{l}\text { FOLFOX } 4 \\
\text { prä- und postop. } \\
\text { vs. alleinige OP }\end{array}$ & $43 \%$ & 83,0 vs. $84 \%$ & $\begin{array}{l}\text { 3-Jhr.-PFS } \\
35,4 \text { vs. } 28,1 \% \\
\text { HR: } 0,79(0,62-1,02) \\
\text { p }=0,058\end{array}$ & Ib \\
\hline
\end{tabular}

1 Phase-I/II-Studie, Zielsetzung definitionsgemäß nicht Wirksamkeitsbeleg. Fast ausschließlich Pat. mit synchronen Metastasen. Vergleich mit Standard fraglich, retrospektive Fallzahllegitimation. Pilotphase nicht randomisiert ( $n=6$ vs. 6 ), dann randomisiert ( $n=16$ vs. 14)

2 Zeitpunkt der Analyse 12 Monate nach Abschluss der Rekrutierung, 2 primäre Endpunkte; 2 -Jhr.-tumorbezogenes ÜL und Ansprechrate. Der erste Endpunkt ist nur von 10 Pat. erreicht, davon 6 am Leben; Phase-II-Studie, Generalisierbarkeit fraglich (monozentrisch, sehr hohe Ansprechrate) medianer Follow-up zum Auswertungszeitpunkt 23 Monate $(12-38)$.

${ }^{3}$ Keine Angabe zum R-Status nach Resektion, 151 vs. 152 Patienten potenziell kurativ reseziert. 
Analyse, die nur die tatsächlich resezierten Patienten berücksichtigt, zeigt, dass eine signifikante Verlängerung des progressionsfreien Überlebens durch eine perioperative Therapie erreicht werden kann (33,2 bis 42,4\%; HR: 0,73; [0,55-0,97]; $p=0,025)$. Dem möglichen Nutzen einer perioperativen Therapie ist die signifikant erhöhte perioperative Morbidität im Chemotherapiearm gegenüberzustellen ( 25 vs. $16 \%$ bei alleiniger Operation, $p=0,04$ ), die Gesamtmortalität war nicht unterschiedlich [841]. Neben dem Risiko für eine erhöhte perioperative Morbidität ist bei der Entscheidung für einen neoadjuvanten Ansatz zu bedenken, dass eine vorangegangene perioperative Chemotherapie die Therapieoptionen im Rezidiv reduziert, dass ein gewisses Risiko besteht, das optimale Zeitfenster für eine Resektion zu verpassen und dass sämtliche Kombinationsprotokolle eine nicht unerhebliche Schädigung des gesunden Lebergewebes verursachen (s. Abschnitt 9.2.1.2.2). Als potenzieller Vorteil wird die Möglichkeit einer frühen Behandlung von Mikrometastasen und der Evaluierung des Ansprechens auf eine Chemotherapie, die hinsichtlich der Einschätzung der Prognose und der postoperativen Planung hilfreich sein kann, diskutiert [842]. In den Leitlinien des US-Amerikanischen National Comprehensive Cancer Network (NCCN) wird deshalb empfohlen, bei synchronen, resektablen Metastasen eine neoadjuvante Therapie (plus adjuvante Therapie postoperativ) als Option neben dem rein adjuvanten Ansatz zu erwägen. Bei metachroner Metastasierung wird primär die Operation empfohlen, wenn der Patient/die Patientin innerhalb der letzten 12 Monate bereits eine Chemotherapie erhalten hat (National Comprehensive Cancer Network. Clincal Guidelines in Oncology: Colon Cancer V2.2007. Verfügbar: „http:// www.nccn.org“, Zugriff am 4.6.2007).

Bei Abwägung der potenziellen Risiken der neoadjuvanten Therapie erscheinen die einschränkende Empfehlung und das Anstreben der zeitnahen Resektion gerechtfertigt.

Das Konzept der perioperativen Chemotherapie primär resektabler Lebermetastasen wird in derzeit laufenden Studien mit Chemotherapieregimen unterschiedlicher Intensität weiter untersucht.

\subsection{Adjuvante Therapie resektabler Lebermetastasen}

\subsection{Empfehlung (2008)}

Nach R0-Resektion synchroner oder metachroner Lebermetastasen kann eine adjuvante Chemotherapie erwogen werden.

Empfehlungsgrad 0, Level of Evidence 2, starker Konsens

\section{Hintergrund}

Trotz R0-Resektion von Lebermetastasen bleiben nur ca. 30\% der Patienten langfristig rezidivfrei. Die Rationale für eine systemische adjuvante Therapie nach Metastasenresektion basiert auf indirekter Evidenz, abgeleitet von Studien, die eine Wirksamkeit einer adjuvanten Chemotherapie bei KRK im Stadium III belegen. Die Datenlage zur systemischen adjuvanten Therapie nach Metastasenresektion ist dagegen limitiert, es liegen keine placebokontrollierten/verblindeten Studien vor (auch nicht zur neoadjuvanten Fragestellung). In zwei randomisierten Studien und der nachfolgenden gepoolten Analyse der erhobenen Daten wurde die Wirksamkeit einer 5-FU-Monotherapie geprüft [846, 847]* [848] ( $\bullet$ Tab. 13). Beide Studien erreichten keine ausreichende Rekrutierung, um einen signifikanten Effekt der Chemotherapie auf das Überleben zu belegen und wurden vorzeitig abgebrochen. Die Interimsanalyse der Studienergebnisse zeigt einen Trend zur Verbesserung des progressionsfreien Intervalls in der einen Arbeit und eine grenzwertig signifikante Verbesserung des progressionsfreien Überlebens in der zweiten Studie. Das Gesamtüberleben wird durch eine adjuvante Therapie nicht beeinflusst. Hierzu ist anzumerken, dass in beiden Studien ein nur wenig effektives Chemotherapieregime (5-FU-Bolusapplikation) eingesetzt wurde. Eine aktuelle Studie, die ein effektiveres Protokoll mit alleiniger Operation verglichen hat, wurde wegen mangelhafter Rekrutierung vorzeitig abgebrochen (ADHOC-Studie). Nach dem mittlerweile vor allem in den USA weit verbreiteten Einsatz der adjuvanten Therapie nach Metastasenresektion wird die Frage, ob eine systemische Chemotherapie einen Vorteil gegenüber einer alleinigen Operation für die Patienten brächte, wahrscheinlich nicht mehr abschließend geklärt werden können. Hinweise, die die Entscheidung für eine systemische adjuvante Therapie stützen, ergeben sich aus der im vorherigen Kapitel zitierten EORTC-Studie und aus einer retrospektiven Analyse von Registerdaten zweier Referenzzentren (Memorial Sloan-Kettering Cancer Center und Royal Infirmary of Edinburgh). Über einen Zeitraum von 8 Jahren (1991-98) wurden dort alle Patienten, die eine Leberresektion aufgrund kolorektaler Karzinommetastasen erhielten, erfasst $(n=792)$ und ein Vorteil für eine 5-FU-basierte, adjuvante Chemotherapie im Vergleich zur alleinigen Operation hinsichtlich des Gesamtüberlebens festgestellt ( $\mathrm{n}=274$ vs. 518; medianes ÜL 47 vs. 36 Monate, 5-Jahres-ÜLR 37 vs. $31 \%$; p =0,007) [849]. In den Leitlinien des US-Amerikanischen National Comprehensive Cancer Network und in nationalen Leitlinien aus Australien wird vor diesem Hintergrund mit Hinweis auf bzw. Einschränkung durch die limitierte Datenlage eine adjuvante Chemotherapie nach Metastasenresektion empfohlen. [National Comprehensive

Tab. 13 Prospektive Studien zur adjuvanten Therapie nach R0-Resektion kolorektaler Lebermetastasen.

\begin{tabular}{|c|c|c|c|c|c|}
\hline Referenz & $\mathbf{n}$ & Therapieregime & Überleben & $\begin{array}{l}\text { Mediane Beob- } \\
\text { achtungszeit }\end{array}$ & Evidenzlevel \\
\hline Portier 2006 & 173 & 5-FU/FS vs. Beobachtung & DFS: 33,5 vs. $26,7 \%(p=0,028)$ OS: 51 vs. $41 \%(p=0,13)$ & $87 \mathrm{mo}$ & Ila \\
\hline Langer $2002^{1}$ & 129 & 5-FU/FS vs. Beobachtung & DFS: 39 vs. 20 Mo ( $p=0,35)$ OS: 53 vs. 43 mMo $(p=0,39)$ & n. a. & - \\
\hline Mitry 20061,2 & 302 & 5-FU/FS vs. Beobachtung & $\begin{array}{l}\text { PFS } 2,2 \text { vs. } 1,55 \text { Jahre }(p=0,059) \text { OS } 5,09 \text { vs. } 3,91 \text { Jahre } \\
(p=0,125)\end{array}$ & n. a. & - \\
\hline $\begin{array}{l}\text { Figueras } 2001 \\
\text { [850] }\end{array}$ & 235 & Vor allem 5-FU/FS & DFS: 34 Mo 5-Jhr.-OS $36 \%$ & $20 \mathrm{mo}$ & III \\
\hline Parks $2007^{3}$ & 792 & 5-FU-basiert vs. Beobachtung & Verbessertes ÜL mit adjuvanter CTX ( $p=0,007$, log rank) & $1991-1998$ & IIIb \\
\hline
\end{tabular}

${ }^{1}$ Nur als Abstract vorliegend; entsprechend eingeschränkte Beurteilbarkeit.

2 Die Zusammenfassung in Mitry et al. ist eine gepoolte Analyse der Arbeiten von Langer und Portier.

${ }^{3}$ Eingeschränkte Aussagekraft von Registerdaten. 
Cancer Network. Clincal Guidelines in Oncology: Colon Cancer V2.2007. Verfügbar: www.nccn.org Zugriff am 4.6.2007; Australian Cancer Network Colorectal Cancer Guidelines Revision Committee. Guidelines for the Prevention, Early Detection and Management of Colorectal Cancer. The Cancer Council Australia and Australian Cancer Network, Sydney 2005. Verfügbar unter www.cancer.org. au, Zugriff am 4.6.2007].

Klinische Gruppen II und III - Indikation für eine systemische Chemotherapie - Allgemeine Empfehlungen

\subsection{Empfehlung (2008)}

Eine medikamentöse Tumortherapie ist grundsätzlich indiziert, da ein Überlebensvorteil nachgewiesen ist.

Empfehlungsgrad A, Level of Evidence 1a, starker Konsens

\section{Hintergrund}

Anfang der 90er Jahre konnte in zwei prospektiv randomisierten Studien mit insgesamt 223 Patienten ein Überlebensvorteil einer systemischen Chemotherapie gegenüber best supportive care (BSC) nachgewiesen werden. So zeigte ein prospektiv randomisierter Vergleich von best supportive care mit einer Chemotherapie mit Bolus 5-FU/FS und Cisplatin bei 40 Patienten ein medianes Gesamtüberleben von 5 Monaten gegenüber 11 Monaten bei Erhalt einer Chemotherapie $(p=0,006)$ [851]. In den Arbeiten der NGTACG lag das mediane Gesamtüberleben bei 14 vs. 9 Monaten in der Kontrollgruppe $(\log \operatorname{rank} \mathrm{p}=0,13)$ [852].

\subsection{Empfehlung (2008)}

Besteht die Indikation zu einer medikamentösen Tumortherapie, so soll diese zum Zeitpunkt des Nachweises der Metastasen unabhängig von metastasenbezogenen Symptomen eingeleitet werden. Bei der Indikationsstellung sind mögliche Kontraindikationen zu berücksichtigen. Alter per se stellt keine Kontraindikation dar. Empfehlungsgrad A, Level of Evidence 1a, starker Konsens

\section{Hintergrund}

Grundsätzlich ist auch in einer primär nicht kurativen Situation zu prüfen, ob sich nach einer medikamentösen Vorbehandlung ein kurativer Ansatz ergeben kann. Bei Metastasierung ist eine Chemotherapie daher unabhängig vom Vorliegen metastasenbezogener Symptome indiziert. Die Wahl der Chemotherapie ist abhängig von der therapeutischen Zielsetzung, d. h. davon, ob eine sekundäre Resektabilität angestrebt oder eine rein palliative/ symptomorientierte Behandlung intendiert ist. Bei Patienten mit marginal resektablen Metastasen soll die Therapie maximal remissionsinduzierend sein (effektivste verfügbare Kombinationstherapie), Patienten mit tumorbedingten Symptomen, Organkomplikationen oder raschem Progress sollte ebenfalls eine möglichst effektive Therapie angeboten werden (s. Gruppe 2). Patienten ohne tumorbedingte Symptome oder Organkomplikationen und/oder mit schwerer Komorbidität (s. Gruppe 3). können auch mit einer weniger intensiven Therapie behandelt werden. Monotherapien, z. B. mit einem Fluoropyrimidin, sind hier zu Beginn möglich. Primäres Therapieziel ist eine Verlängerung des progressionsfreien und des Gesamtüberlebens bei bestmöglicher Lebensqualität.

Die meisten Patienten sind bei Erstdiagnose älter als 65 Jahre. Trotzdem wurden bis vor wenigen Jahren nur wenige Patienten über 70 Jahre in randomisierten Studien untersucht. Einige Arbeiten der letzten Jahre haben sich mit der Frage einer Tumortherapie bei älteren Patienten mit KRK auseinandergesetzt. So konnte für das FOLFOX-Regime gezeigt werden, dass ältere Studienpatienten in gleicher Weise wie jüngere Patienten bez. Remissionsrate und progressionsfreiem sowie Gesamtüberleben von einer intensivierten Therapie profitieren, wenngleich vor allem die Hämatotoxizität etwas erhöht ist (Grad-3-Neutropenie 43 vs. $49 \%$ p = 0,04, Thrombopenie 2 vs. $5 \%, p=0,04$ ) [740]. Mattioli zeigte für ein bifraktioniertes FOLFOX-Protokoll eine hohe Wirksamkeit bei einer Patientengruppe mit einem Altersmittel von 75 Jahren [853]. Feliu untersuchte in einer Phase-II-Studie die Verträglichkeit von CAPOX bzw. Capecitabin mono in der Erstlinientherapie bei Patienten, die älter als 70 Jahre waren [854]. Eine spanische Arbeitsgruppe behandelte eine Auswahl älterer Patienten über 72 Jahre mit FOLFIRI [855], eine exploratorische Subgruppenanalyse der BICC - C Studie an Patienten die älter als 65 Jahre alt waren ergab keinen Unterschied in Wirksamkeit und Toxizität eines irinotecanhaltigen Protokolls im Vergleich mit jüngeren Patienten [856]. Eine in diesem Jahr publizierte Analyse randomisierter Studien konnte eine Verbesserung der Ansprechraten bei einer irinotecanbasierten Chemotherapie sowohl bei jüngeren als auch bei älteren Patienten ( $>70$ Jahre) zeigen (46,6 vs. $29,0 \%, p<0,0001$; und 50,5 vs. $30,3 \%$, $\mathrm{p}<0,0001$ ). Das Gleiche galt für das PFS (HR 0,77; $95 \%$ CI, 0,70 0,$85 ; \mathrm{p}<0,0001$ für jüngere Patienten resp.HR 0,75; $95 \% \mathrm{Cl}$, $0,61-0,90 ; p=0,0026$ für Patienten $>70$ Jahre) und mit Einschränkung für das Gesamtüberleben, wo bei den älteren Patienten ein Trend zur Verbesserung beobachtet werden konnte (HR 0,83; $95 \%$ CI, 0,75-0,92; p=0,0003 und HR, 0,87; $95 \%$ CI, 0,72-1,05; $\mathrm{p}=0,15$ für Patienten > 70 Jahre) [857]. Bei Patienten über 80 Jahren ist die Datenlage weiterhin spärlich. Auch ältere Patienten sollten somit bei entsprechender Indikation eine Chemotherapie erhalten. Bei der Indikationsstellung und Auswahl der geeigneten Therapie gilt es die Veränderung von Organfunktionen im Alter, eventuell vorliegende Komorbiditäten sowie altersbedingte Einschränkungen des funktionellen Status zu berücksichtigen.

Zur tumorspezifischen Palliativbehandlung bei inoperabler Metastasierung stehen neben der Chemotherapie chirurgische, interventionelle (endoskopische, radiologische) und strahlentherapeutische Maßnahmen zur Verfügung, auf deren Einsatzmöglichkeiten in den entsprechenden Kapiteln näher eingegangen wird.

\subsection{Empfehlung (2008)}

Bei Indikation zur systemischen Therapie (z. B. inoperable Leber-/ Lungenfiliae) kann der Primärtumor belassen werden. Ausnahmen können ein symptomatisch stenosierendes Tumorwachstum und/ oder eine Hb-relevante Blutung sein.

Empfehlungsgrad 0, Level of Evidence 4, starker Konsens

\subsection{Empfehlung (2008)}

Grundsätzlich sollen die Patienten im Laufe ihrer Therapie Zugang $z u$ allen verfügbaren Medikamenten haben.

Empfehlungsgrad A, Level of Evidence 5

\subsection{Patienten mit einer Indikation für eine intensivierte systemische Therapie}

9.2.1. Patienten mit potenziell resektablen Metastasen

Definition: $\mathrm{Zu}$ dieser nach klinischen Kriterien definierten Patientengruppe gehören Patienten mit Leber- und/oder Lungenmetastasen, die, zunächst als irresektabel bzw. marginal resektabel eingestuft, nach Ansprechen auf eine neoadjuvante Therapie resektabel werden. 
Tab. 14 Ansprechraten und Überleben von Patienten nach Erreichen einer sekundären Resektabilität (ergänzt nach [862]).

\begin{tabular}{|c|c|c|c|c|c|c|}
\hline Referenz & $\mathbf{n}$ & Therapieregime & Ansprechrate (\%) & $\begin{array}{l}\text { R0-Resektionsrate } \\
\text { (\%) }\end{array}$ & $\begin{array}{l}\text { Langzeit } \\
\text { überleben } \\
\text { der res. Pat. }\end{array}$ & $\begin{array}{l}\text { Evidenz- } \\
\text { level }\end{array}$ \\
\hline Falcone $2007^{1}$ & 244 & FOLFIRI vs. FOLFOXIRI & $\begin{array}{l}34 \text { vs. } 60 \\
(p<0,0001)\end{array}$ & $\begin{array}{l}6 \text { vs. } 15 \\
(p=0,033)\end{array}$ & - & $\mathrm{Ib}$ \\
\hline $\begin{array}{l}\text { Van Cutsem } 2007 \\
\text { (CRYSTAL) }^{2}\end{array}$ & 1198 & $\begin{array}{l}\text { FOLFIRI vs. FOLFIRI + Cetu- } \\
\text { xuimab }\end{array}$ & $\begin{array}{l}38,7 \text { vs. } 46,9 \\
(p=0,0038)\end{array}$ & $\begin{array}{l}1,5 \text { vs. } 4,3 \\
(p=0,0034)\end{array}$ & - & $\mathrm{Ib}$ \\
\hline Adam $2001^{3}$ & 701 & Oxa+FU/FS (chron) & PCR: 6,3 & 13,5 & 5-JÜL: $35 \%$ & $\mathrm{IIb}$ \\
\hline Giacchetti $2006^{4}$ & 564 & $\begin{array}{l}\text { FOLFOX2 vs. LOHP } 5 F U \text { FS } \\
\text { chron. }\end{array}$ & 44 vs. 42 & $\begin{array}{l}\text { R0: } 12,4 \text { vs. } 13,1 \\
\text { PCR: } 1,1 \text { vs. } 2,8\end{array}$ & - & IV \\
\hline $\begin{array}{l}\text { Tournigand } \\
20064\end{array}$ & 620 & $\begin{array}{l}\text { FOLFOX } 4 \text { vs. FOLFOX } 7+ \\
\text { Erhaltung }\end{array}$ & $\begin{array}{l}58,5 \text { vs. } 59,2 \\
\text { n. s. }\end{array}$ & 11,3 vs. 9,4 & $\begin{array}{l}38,9 \text { vs. } 43 \text { Mo } \\
(p=0,93)\end{array}$ & IV \\
\hline Souglakos 20064 [863] & 283 & FOLFOXIRI vs. FOLFIRI & $\begin{array}{l}43 \text { vs. } 33,6 \\
(p=0,168)\end{array}$ & 8,8 vs. 3,4 & - & IV \\
\hline Saltz 20084 [864] & 1401 & $\begin{array}{l}\text { XELOX/FOLFOX } 4 \text { + Beva vs. } \\
\text { XELOX/FOLFOX } 4 \text { + Placebo }\end{array}$ & $\begin{array}{l}38 \text { vs. } 38 \\
(p=0,99)\end{array}$ & n.a. & $\begin{array}{l}21,3 \text { vs. } 19,9 \text { Mo } \\
(p=0,077)\end{array}$ & IV \\
\hline
\end{tabular}

${ }^{1}$ Resektionsrate sekundärer Endpunkt, FOLFOXIRI als kontinuierliche Infusion, höhere Dosen der Chemotherapie im FOLFOXIRI-Arm als bei Souglakos et al.

2 Resektionsrate sekundärer Endpunkt, Benefit nur bei Behandlung von Patienten mit k-ras-Wildtyp-Tumoren.

${ }^{3}$ Prospektive Beobachtungsstudie, Resektionsrate primärer Endpunkt, potenziell kurativ operierte Patienten, R0-Status nicht berichtet

${ }^{4}$ Resektionsraten als Ergebnis exploratorischer Subgruppenanalysen.

\subsubsection{Vorgehen bei isolierten primär irresektablen} Lungenmetastasen

\subsection{Empfehlung (2008)}

Bei primärer Irresektabilität soll eine systemische Chemotherapie erfolgen.

Empfehlungsgrad A, Level of Evidence 4, starker Konsens

\subsubsection{Vorgehen bei isolierten primär irresektablen} Lebermetastasen

\subsection{Systemische neoadjuvante Therapie}

\subsection{Empfehlung (2008)}

Bei primär irresektablen Lebermetastasen soll eine systemische Therapie begonnen werden. Wichtig ist die regelmäßige Evaluation einer möglichen sekundären Resektabilität nach Remissionsinduktion. Ist das Therapieziel die Remissionsinduktion mit sekundärer Metastasenresektion, dann soll primär die effektivste jeweils verfügbare systemische Kombinationstherapie angewandt werden (intensivierte Therapie).

Empfehlungsgrad A, Level of Evidence 4, starker Konsens

\section{Hintergrund}

Ca. 35\% aller Patienten mit kolorektalem Karzinom weisen bei Diagnosestellung Metastasen auf. 15-20\% der synchronen und metachronen Metastasen können in kurativer Intention reseziert werden. In den meisten Fällen werden die Metastasen jedoch aus verschiedenen Gründen als primär irresektabel eingestuft. Die Möglichkeit, mit einer systemischen Chemotherapie ein Downsizing primär irresektabler Lebermetastasen und so eine sekundäre Resektabilität und potenzielle Heilung zu erreichen, wurde in mehreren Arbeiten als primärer/sekundärer Endpunkt und im Rahmen exploratorischer Subgruppenanalysen von Studien mit primär palliativer Intention untersucht ( $\bullet$ Tab. 14).

Eine retrospektive Analyse von Giacchetti und Bismuth zeigte ein 5-Jahres-Gesamtüberleben von ca. 50\% nach neoadjuvanter Chemotherapie und anschließender Resektion, vergleichbar den Langzeitergebnissen nach primärer Resektion von Lebermetastasen bei Patienten mit einem FONG-Score < 3 [858]. Für manche Chemotherapieregime sind besonders gute Ansprech- und Resektionsraten beschrieben. So konnte Falcone in seiner 2007 pub- lizierten Arbeit im Vergleich von FOLFIRI mit FOLFOXIRI eine signifikante Verbesserung der Ansprechraten (34 vs. $60 \%$, p < 0,001) und der R0-Resektionsrate erreichen ( 6 vs. $15 \%, p=0,033$ ). Dieser Vorteil wurde bei den Patienten mit isolierten Lebermetastasen noch deutlicher (12 vs. $36 \%, \mathrm{p}=0,017$ ) [859].

Mit der Kombination aus FOLFIRI und dem EGFR-Antikörper Cetuximab wurde in einem Phase-III-Design eine ORR von 46,9\% (vs. 38,7\% für FOLFIRI alleine) erreicht. Der Anteil an R0-Resektionen als sekundärer Endpunkt war im experimentellen Arm erhöht (4,3 vs. 1,5\%) [860]. Den größten Benefit erreichten Patienten mit einem k-ras Wildtyp exprimierendem Tumor. In dieser retrospektiven Analyse wurde bei Cetuximab behandelten Patienten mit k-ras Wildtyp exprimierendem Tumor ein statistisch signifikanter Unterschied für das PFS gesehen ( $p=0,0167$; HR: 0,68 [95\% CI: 0,051 - 0,934]) aber auch die Ansprechraten waren deutlich verbessert (59,3\% [Cetuximab + FOLFIRI] vs. 43,2\% [FOLFIRI], $\mathrm{p}=0,0025$ ) [861 $]^{*}$.

Bei Patienten mit isolierten Lebermetastasen gibt es eine signifikante Korrelation von Remissionsrate und Resektionsrate $(r=0,96, p=0,002)$. So korrelierte in großen Studien mit primär palliativer Intention und einem unselektionierten Patientenkollektiv die Ansprechrate ebenfalls mit der Resektionsrate $(r=0,74$, $\mathrm{p}<0,001$ ). Bei der Interpretation der Studiendaten gilt es, die Patientenselektion und die zwischen den einzelnen Studien häufig nicht einheitliche Definition von Resektabilität zu beachten [862].

\subsection{Chemotherapiefolgen auf das gesunde Lebergewebe und Metastasenlokalisation}

\subsection{Empfehlung (2008)}

Die Hepatotoxizität o. a. Protokolle z. B. „Blue Liver“/chemotherapieassoziierte Steatohepatitis (CASH) sollte dabei in die differenzialtherapeutische Entscheidung und OP-Planung miteinbezogen werden.

Empfehlungsgrad B, Level of Evidence 3, starker Konsens

\section{Hintergrund}

Mehrere Arbeitsgruppen haben sich in den vergangenen Jahren mit der Frage befasst, inwieweit eine präoperative Chemothera- 
pie das Risiko von Komplikationen bei einer Leberteilresektion beeinflusst. Aloia und Mitarbeiter untersuchten eine Kohorte von 303 Patienten, bei denen eine Leberteilresektion aufgrund kolorektaler Lebermetastasen durchgeführt worden war. $92 \mathrm{~Pa}-$ tienten wurden zufällig ausgewählt, 75 hatten eine präoperative Chemotherapie erhalten, 17 Patienten nicht. Diejenigen, bei denen eine präoperative Chemotherapie durchgeführt worden war, benötigten signifikant häufiger intraoperative Transfusionen. Die prädominierenden histopathologischen Veränderungen des gesunden Lebergewebes waren vaskuläre Läsionen im Sinne eines sinusoidalen Obstruktionssyndroms (SOS) und korrelierten - bei schwerer Ausprägung - positiv mit dem Bedarf an intraoperativen Transfusionen. Die postoperative Morbidität war abhängig von der Dauer der präoperativen Chemotherapie [865]. In einer weiteren retrospektiven Arbeit erhielten 61\% der untersuchten Patienten eine präoperative Chemotherapie. Eine Therapie mit Oxaliplatin ging häufiger mit einer sinusoidalen Obstruktion im nicht befallenen Lebergewebe ohne nennenswerte Mortalität einher (1,6\%). Eine irinotecanhaltige Therapie war dagegen eher mit einer Steatohepatitis assoziiert. Patienten mit Steatohepatitis wiesen eine höhere 90-Tages-Mortalität als solche ohne Steatohepatitis auf (14,7 vs. 1,6\%) [866].

\subsection{Empfehlung (2008)}

Intraoperativ sollte eine Exploration der Leber anhand der Metastasenlokalisation in der Ausgangs-Bildgebung erfolgen. Es sollte eine chirurgische Therapie aller Ausgangsläsionen angestrebt werden.

Empfehlungsgrad B, Level of Evidence 3b, starker Konsens

\section{Hintergrund}

Eine aktuelle Arbeit von Benoist et al. zeigte, dass trotz einer radiologisch in der CT konstatierten kompletten Remission nach RECIST Kriterien in $83 \%$ der Fälle entweder makroskopisch oder mikroskopisch noch residuales Tumorgewebe nachweisbar war oder innerhalb eines Jahres in situ ein Rezidiv auftrat. Eingeschlossen wurden 38 Patienten mit insgesamt 66 Lebermetastasen. Daher sollte die Metastasenresektion zum frühestmöglichen Zeitpunkt einer möglichen R0-Resektion erfolgen und sich an den primären Grenzen der vor Therapie vorhandenen Läsionen orientieren [867].

\subsection{Lokoregionäre Therapieverfahren \\ Radiofrequenzablation (RFA)}

\subsection{Empfehlung (2013)}

Eine RFA kann durchgeführt werden, wenn nicht resektable Lebermetastasen vorliegen oder der Allgemeinzustand des Patienten eine Resektion nicht zulässt, insbesondere nach vorangegangener Leberresektion.

Empfehlungsgrad 0, Level of Evidence 3a, de novo: [586-588], starker Konsens

\section{Hintergrund}

Die derzeitige Evidenzlage zur Sicherheit und Effektivität der Radiofrequenzablation bei kolorektalen Lebermetastasen ist angemessen, um die Verwendung dieser Methode bei Patienten zu befürworten bei denen entweder nicht resektable Lebermetastasen vorliegen, deren Zustand eine Resektion nicht zulässt, oder bei denen zuvor eine Leberresektion durchgeführt worden ist [586]. Die RFA kann auch primär in Kombination mit der chirurgischen Resektion durchgeführt werden.
Neuere Arbeiten legen die Vermutung nahe, dass bei solitären Lebermetastasen $<3 \mathrm{~cm}$ mittels RFA ähnlich gute Ergebnisse wie durch die Resektion erzielt werden können [587, 588]. Die bisherige Datenlage hierzu ist aber widersprüchlich und es fehlen weiterhin vergleichende kontrollierte-randomisierte Studien.

\section{Selective Internal Radiation Therapy (SIRT)}

\subsection{Empfehlung (2013)}

Eine SIRT zur Behandlung von disseminierten Lebermetastasen bei KRK sollte nur bei Patienten, für die keine andere Therapieoption infrage kommt, und dann nur innerhalb klinischer Studien durchgeführt werden.

Empfehlungsgrad B, Level of Evidence 2a, de novo: [589, 590], Konsens

\section{Hintergrund}

Patienten mit fehlender oder allenfalls begrenzter extrahepatischer Metastasierung, die zudem systemtherapeutisch austherapiert sind, zeigen in einzelnen Studien mit SIRT (gleichbedeutend mit dem Begriff Radioembolisation) ein verlängertes medianes Überleben sowie ein längeres Intervall bis zum Progress von Lebermetastasen. Für eine endgültige Bewertung, insbesondere auf Überleben und Lebensqualität liegen zu wenige Daten vor. Deshalb sollten Patienten, die für eine SIRT infrage kämen, nur innerhalb klinischer Studien behandelt werden. [589, 590].

\section{Laserinduzierte interstitielle Thermotherapie (LITT)}

\subsection{Empfehlung (2013)}

Eine LITT zur Behandlung von Lebermetastasen bei KRK sollte nur innerhalb klinischer Studien durchgeführt werden.

Empfehlungsgrad B, Level of Evidence 4, de novo: [591, 592], starker Konsens

\section{Hintergrund}

In einzelnen Fallserien war die interstitielle Laserthermoablation eine sichere und wirksame Behandlung für Patienten mit inoperablen Lebermetastasen kolorektaler Karzinome [591, 592]. Daten zum Effizienzvergleich mit der perkutanen Radiofrequenzablation liegen nicht vor. Für eine endgültige Bewertung, insbesondere auf Überleben und Lebensqualität liegen zu wenige Daten vor. Deshalb sollten Patienten, die für eine LITT infrage kämen, nur innerhalb klinischer Studien behandelt werden.

\subsubsection{Patienten mit einer Indikation für eine intensivierte} palliative Therapie

Das Vorgehen in dieser Patientengruppe entspricht im Wesentlichen dem in Abschnitt 9.2.1 beschriebenen.

\subsection{Empfehlung (2008)}

Patienten mit tumorbedingten Symptomen, Organkomplikationen oder raschem Progress sollten unter Berücksichtigung des Allgemeinzustands des Patienten eine möglichst effektive Kombinationstherapie erhalten (intensivierte Therapie).

Empfehlungsgrad B, Level of Evidence 5, starker Konsens

\subsection{Patienten mit der Möglichkeit für eine weniger intensive Therapie}

Patienten mit multiplen Metastasen ohne Option für Resektion nach Metastasenrückbildung ohne tumorbezogene Symptome oder Organkomplikationen und/oder schwerer Komorbidität. 
Primäres Therapieziel in dieser Patientengruppe ist weniger die Remissionsinduktion als eine Verlängerung des progressionsfreien und des Gesamtüberlebens bei geringer Toxizität und guter Lebensqualität.

\subsection{Empfehlung (2008)}

Bei Patienten mit multiplen Metastasen ohne Option für Resektion nach Metastasenrückbildung ohne tumorbezogene Symptome oder Organkomplikationen und/oder schwerer Komorbidität kann eine Monotherapie als Erstlinientherapie eingesetzt werden.

Empfehlungsgrad 0, Level of Evidence 1, starker Konsens

\section{Hintergrund}

Mehrere Arbeiten beschäftigen sich mit der Frage nach einer optimalen Sequenz der verschiedenen Chemotherapieprotokolle in der Behandlung des kolorektalen Karzinoms. Die CAIRO-Studie untersuchte die Frage, ob eine sequenzielle Monotherapie, einer initialen Kombinationstherapie bezüglich des Gesamtüberlebens gleichwertig ist. 820 Patienten wurden in einen der beiden Therapiearme randomisiert und entweder mit der sequenziellen Monotherapie (bestehend aus Capecitabin $\rightarrow$ Irinotecan $\rightarrow$ CAPOX) oder mit der Kombinationstherapie behandelt (CAPIRI $\rightarrow$ CAPOX). Hierbei bestand, bei vergleichbarer Toxizität, kein signifikanter Unterschied im Gesamtüberleben (16,3 vs. 17,4 Monate, p=0,3281) [868]. Die Ergebnisse dieser Studie sind jedoch im Hinblick auf Studiendesign und Patientenselektion zurückhaltend zu betrachten und nicht uneingeschränkt übertragbar [869].

Der FOCUS-Trial, die mit insgesamt 2135 Patienten größte Einzelstudie beim kolorektalen Karzinom, wies nach, dass eine Kombinationstherapie als Erst- und/oder Zweitlinientherapie einer Sequenz von Monotherapien (Arm A) überlegen ist. Der Beginn mit einer 5FU-Monotherapie gefolgt von 5-FU in Kombination mit entweder Oxaliplatin oder Irinotecan (Arm B) resultierte in einem Gesamtüberleben von 15,2 und 15,0 vs. 13,9 Monate in $\operatorname{Arm~A~(~} p=0,24)$, bei einer Kombinationstherapie als Erst- und Zweitlinientherapie (Arm C) erreichte der Vorteil im Gesamtüberleben insgesamt statistische Signifikanz. (FOLFOX 15,4 Monate, FOLFIRI 16,7 Monate vs. 13,9 Monate, $p=0,02$ ) [870]. Ein weiteres Argument gegen den generellen Einsatz einer Monotherapie als Erstlinientherapie liefert eine aktuell publizierte retrospektive Subgruppenanalyse der N9741 Studie. Dort wird das Erreichen einer kompletten Remission mit einem verbesserten Gesamtüberleben assoziiert, Die Rate an kompletten Remissionen nach 5-FU-Monotherapie liegt nur bei ca. $1 \%$, wohingegen nach FOLFOX4 komplette Remissionsraten von $6,2 \%$ beschrieben werden [871]. Zusammenfassend ist zu sagen, dass eine möglichst aktive Therapie als Erstlinientherapie gegeben werden sollte, eine 5-FU-Monotherapie mit nachfolgender Kombinationstherapie aber in bestimmten Fällen (z. B. Gruppe 3) eine vertretbare Alternative darstellt und mit den Patienten besprochen werden sollte. Eine sequenzielle Monotherapie (z. B. 5-FU-Monotherapie gefolgt von Irinotecan-Monotherapie) kann auf Grundlage dieser Daten nicht empfohlen werden. Die Therapiesequenz 5-FU Bolus gefolgt von 5-FU-Infusionsprotokollen, ohne Erweiterung durch eine zweite oder evtl. dritte Substanz ist obsolet. Wichtig scheint, dass Patienten im Lauf ihrer Therapie Zugang zu allen aktiven Substanzen haben [872]. Der Stellenwert biologischer Substanzen innerhalb des jeweiligen onkologischen Gesamtkonzepts wird in den entsprechenden Abschnitten besprochen.

\subsection{Chemotherapieprotokolle}

\subsubsection{Erstlinientherapie}

Die Optionen für die Behandlung des metastasierten kolorektalen Karzinoms sind durch die Einführung neuer Chemotherapeutika wie Irinotecan und Oxaliplatin, der oralen 5-FU-Prodrugs und später der biologischen Substanzen deutlich verbessert worden. Als Therapieoptionen stehen für die Erstlinientherapie Monotherapien und fluoropyrimidinbasierte Kombinationstherapien mit Oxaliplatin und/oder Irinotecan zur Verfügung. Bei denjenigen Patienten, bei denen eine intensivierte Therapie notwendig ist und die dafür qualifizieren, ist eine Monotherapie zu Beginn nicht indiziert. Die Wahl des Therapieregimes orientiert sich dabei am jeweiligen Therapieziel, d. h. Erreichen einer guten Remission und möglicherweise sekundären Resektabilität oder der Verlängerung des progressionsfreien Überlebens und des Gesamtüberlebens bei guter Lebensqualität. Bei der Entscheidung ist das spezifische Nebenwirkungsprofil der einzelnen Chemotherapeutika, etwaige Komorbiditäten (z. B. KHK, chronische Diarrhö), aber auch die persönliche und berufliche Lebenssituation des Patienten zu beachten. Bei Toxizität soll gemäß dem üblichen onkologischen Vorgehen das toxische Agens pausiert werden. Wenn eine initiale medikamentöse Therapie z. B. nach Erreichen einer „best response“ oder intolerabler Nebenwirkungen deeskaliert wurde, sollte bei Progress zunächst die initiale Therapie wieder aufgenommen werden, sofern die Toxizität dies zulässt (z. B. in Analogie zum Optimox-Schema). Ist dies nicht der Fall, soll ein alternatives Therapieschema zum Einsatz kommen. Dies gilt für Mono- und Kombinationstherapien. Hingegen sollte bei Progress unter oder relativ kurzfristig nach einer Primärtherapie auf ein alternatives Therapieprotokoll gewechselt werden.

\subsubsection{Monotherapie (5-FU)}

\subsection{Empfehlung (2008)}

Für den Fall, dass eine Fluoropyrimidin-Monotherapie gegeben wird, sollte eine orale der intravenösen 5-FU-Gabe vorgezogen werden. Bei den zur Auswahl stehenden Infusionsprotokollen sollte dem de Gramont-Schema der Vorzug gegenüber dem AIO-Schema gegeben werden, da das de Gramont-Schema bei wahrscheinlich gleicher Wirksamkeit durch die 14-tägige Applikation eine geringere Belastung für den Patienten darstellt.

Empfehlungsgrad B, Level of Evidence 4, Konsens

\section{Hintergrund}

5-FU war seit den 50er Jahren das Standardtherapeutikum in der Behandlung des kolorektalen Karzinoms und führte zu Remissionsraten von $10-15 \%$ und einem medianen Gesamtüberleben von ca. 6-9 Monaten. Ende der 80er-Jahre konnten durch die Kombination von Bolus 5-FU mit Biomodulatoren wie Folinsäure Remissionsraten von etwa $20 \%$ und eine mediane Überlebenszeit von ca. 12 Monaten dokumentiert werden [873, 874]. Randomisierte Studien, die eine 5-FU Bolusapplikation mit einer kontinuierlichen Gabe bis zum Progress verglichen, zeigten höhere Ansprechraten ( 7 vs. $30 \%$ p < 0,001) ohne Einfluss auf das Gesamtüberleben (10,3 vs. 11,3 Monate, $\mathrm{p}=0,379$ ). Die kontinuierliche Gabe zeigte dabei eine höhere Inzidenz an Hand-Fuß-Syndromen (23 vs. $0 \%, p<0,001$ ) aber weniger Grad-3- bis -4-Neutropenien ( 1 vs. $22 \%, p<0,001)$. Im Bolusarm kam es zu 4 Todesfällen durch neutropene Sepsis [875]. De Gramont konnte 1997 bei geringerer Toxizität eine statistisch signifikante Verbesserung der Ansprechraten $(32,6$ vs. $14,5 \%, p=0,0004)$ und des mittleren progressionsfreien Überlebens (27,6 vs. 22 Wochen, $\mathrm{p}=0,001)$ mit einem zweiwöchentlichen infusionalen Protokoll erreichen [876]. Eine 
Tab. 15 Capecitabin (Cape) im Vergleich mit Bolus 5-FU/FS in der Erstlinientherapie.

\begin{tabular}{|c|c|c|c|c|c|c|}
\hline Referenz & $\mathbf{n}=$ & Therapieregime & Ansprechrate (\%) & TTP (Mo) & OS (Mo) & Evidenzlevel \\
\hline Van Cutsem 2004 & 1207 & Cape/Mayo & 26 vs. $17(\mathrm{p}<0,0002)$ & 4,6 vs. $4,7(p=0,95)$ & 12,9 vs. $12,8(p=0,48)$ & la \\
\hline Van Cutsem 2001 & 602 & Cape/Mayo & 18,9 vs. 15 & 5,2 vs. $4,7(p=0,65)$ & 13,2 vs. $12,1(p=0,33)$ & $\mathrm{Ib}$ \\
\hline Hoff 2001 & 605 & Cape/Mayo & 24,8 vs. $15,5(p=0,005)$ & 4,3 vs. $4,7(p=0,72)$ & 12,5 vs. $13,3(p=0,974)$ & $\mathrm{lb}$ \\
\hline
\end{tabular}

Tab. 16 Oxaliplatinhaltige Protokolle in der Erstlinientherapie - Phase-III-Studien.

\begin{tabular}{|c|c|c|c|c|c|c|}
\hline Referenz & $\mathbf{n}$ & Therapieregime & Ansprechrate (\%) & PFS (Mo) & OS (Mo) & Evidenzlevel \\
\hline de Gramont 2000 & 420 & FOLFOX4 vs. 5FU/FS & 50,7 vs. $22,3(p=0,0001)$ & 9,0 vs. $6,2(p=0,0003)$ & 16,2 vs. 14,7 n.s. & $\mathrm{Ib}$ \\
\hline Giacchetti 2006 [889] & 564 & $\begin{array}{l}\text { FOLFOX2 vs. Oxali- } \\
\text { platin/5FU (chrono- } \\
\text { mod) }\end{array}$ & 44,3 vs. 42,0 & $\begin{array}{l}8,4 \text { vs. } 8,4 \\
\text { n. s. }\end{array}$ & 18,7 vs. 19,6 n. s. & $\mathrm{Ib}$ \\
\hline Giacchetti 2000 [890] & 200 & $\begin{array}{l}\text { Oxaliplatin/5FU } \\
\text { vs. } 5 \mathrm{FU} \text { (beides } \\
\text { chronomod) }\end{array}$ & 53 vs. $16(p<0,001)$ & 8,7 vs. $6,1(p=0,048)$ & 19,4 vs. 19,9 & $\mathrm{Ib}$ \\
\hline
\end{tabular}

Metaanalyse aus demselben Jahr bestätigte diesen Vorteil im Gesamtüberleben. Obwohl in den einzelnen Studien nicht signifikant, zeigte die Analyse von sechs Studien mit insgesamt 1219 Patienten einen signifikanten Unterschied im Gesamtüberleben von 12,1 Monaten bei kontinuierlicher 5-FU Gabe gegenüber 11,3 Monaten bei Bolusprotokollen $(p=0,04)$ [877]. Orale Fluoropyrimidine können die Lebensqualität weiter verbessern, da sie eine ambulante Therapie ohne Port-Systeme und Pumpen, die mit einer Komplikationsrate von ca. $10 \%$ assoziiert sind (Thrombose, Infektion, Dislokation) ermöglichen [878 - 880]. Zudem entstehen niedrigere Kosten. Die Nebenwirkungen von Capecitabin sind hauptsächlich das Hand-Fuß-Syndrom, Hämatotoxizität und Diarrhöen. Studien haben gezeigt, dass Patienten die orale Applikationsform bevorzugen, solange die Wirksamkeit nicht kompromittiert ist [881, 882]. Twelves untersuchte 97 Patienten mit mKRK. Im direkten Vergleich mit Capecitabine resultierte nur das modifizierte ambulant verabreichten de Gramont Schema in einer vergleichbaren Patientenzufriedenheit. Der Gewinn an Lebensqualität bei den befragten Patienten war bei dem infusionalen ambulant verabreichten Schema sogar höher $(p<0,05)$ [883]. Die Wirksamkeit von Capecitabin im Vergleich mit einem 5-FU-Protokoll wurde in zwei multizentrischen randomisierten Phase-III-Studien untersucht. Der Kontrollarm bestand jeweils aus einem Bolus 5-FU-Protokoll (Mayo-Protokoll), welches dem damaligen Therapiestandard entsprach. Van Cutsem zeigte Ansprechraten von 18,9\% für Capecitabin und 15\% für 5-FU/FS, das mediane Gesamtüberleben lag bei 13,2 resp. 12,1 Monaten ( $p=0,33$ ). Die Einnahme von Capecitabine führte zu einer geringeren Inzidenz von Grad-3- und -4-Stomatitis und -Neutropenie ( $p<0,00001$ ) aber häufiger zum Auftreten des Hand-FußSyndroms $(p<0,00001)$ [884]. Hoff konnte in seiner Arbeit mit 605 Patienten ähnliche Ergebnisse demonstrieren [885]. Eine im Jahr 2004 publizierte gepoolte Analyse dieser beiden Studien mit insgesamt 1207 Patienten demonstrierte hoch signifikant unterschiedliche Ansprechraten von $26 \%$ bei mit Capecitabin behandelten Patienten vs. $17 \%$ im 5-FU-Arm ( $p<0,0002)$. Das Gesamtüberleben war nicht verlängert. (12,9 vs. 12,8 Monate) [886]. Ein direkter randomisierter Vergleich von Capecitabin mit einem infusionalen 5-FU-Protokoll liegt derzeit nur in Kombination mit Irinotecan/Oxaliplatin mit oder ohne Bevacizumab vor. Capecitabin stellt hier eine wirkungsvolle und gut verträgliche Alternative zu infusionalen 5-FU-Protokollen dar ( $\bullet$ Tab. 15).
Bei Patienten, die eine 5-FU/Folinsäure-basierte Therapie erhielten, konnten als günstige prognostische Faktoren ECOG Performance Status $(0-1)$, Leukozytenzahl $\left(<10 \times 10^{9} / \mathrm{L}\right)$, alkalische Phosphatase $(<300 \mathrm{U} / \mathrm{l})$ und der Nachweis nur einer Metastasenlokalisation identifiziert werden [887].

\subsubsection{Kombinationstherapie}

In den letzten Jahren erschienen eine Reihe großer Phase-III-Studien für die Erstlinientherapie des mKRK, die Patienten in eine Gruppe mit einer Fluoropyrimidin-Monotherapie und eine Gruppe mit einer Kombinationschemotherapie aus Fluoropyrimidin und Irinotecan oder Oxaliplatin randomisierten. Die Kombinationstherapie verbesserte in allen Studien die Ansprechraten und das progressionsfreie Überleben, in 2 der 3 iriontecanbasierten Therapiestudien konnte für die Kombinationstherapie ein signifikanter Überlebensvorteil gezeigt werden, wobei der Einfluss der Zweit- und Drittlinientherapien auf das Gesamtüberleben in der Beurteilung der Wertigkeit einer Erstlinientherapie nicht außer Acht gelassen werden sollte.

\section{Oxaliplatin + 5-FU/Folinsäure}

\section{Hintergrund}

Die Kombination von infusionalem 5-FU/FS mit Oxaliplatin ergab eine signifikante Verlängerung des progressionsfreien Überlebens gegenüber einer alleinigen infusionalen 5-FU Therapie (medianes PFS von 9 Monaten versus 6,2 Monaten bei alleiniger 5-FU/ FS Therapie; $p=0,0003$ ) ( $\bullet$ Tab. 16). Auch die Ansprechraten waren mit der intensiveren Therapie signifikant besser (50,7 vs. $22,3 \%, p=0,0001$ ), allerdings wurden auch mehr Grad-3- bis -4Toxizitäten beobachtet. V.a. Neutropenien und neurologische Komplikationen traten signifikant häufiger im Oxaliplatin-Arm auf ( $p<0,001)$. Das Gesamtüberleben war nicht signifikant verlängert (16,2 vs. 14,7 Monate, $\mathrm{p}=0,12$ ) [888].

\section{Irinotecan + 5-FU/Folinsäure (FS) \\ Hintergrund}

Die Kombination aus Irinotecan und Bolus-5-FU/FS (IFL) weist ein ungünstiges Wirkungs-/Nebenwirkungsverhältnis auf und ist deshalb obsolet.

Der Vergleich einer 5-FU/FS-Monotherapie mit einer Kombinationstherapie mit infusionalem 5-FU/FS und Irinotecan zeigte 
Tab. 17 Irinotecanhaltige Protokolle in der Erstlinientherapie - Phase-III-Studien.

\begin{tabular}{|c|c|c|c|c|c|c|}
\hline Referenz & $\mathbf{n}$ & Therapieregime & Ansprechrate (\%) & PFS (Mo) & OS (Mo) & Evidenzlevel \\
\hline Kohne 2005 & 430 & $\mathrm{AlO}+\mathrm{CPT} 11$ vs. $\mathrm{AlO}$ & 62,2 vs. $34,4(p<0,0001)$ & 8,5 vs. $6,4(p<0,0001)$ & 20,1 vs. 16,9 n. s. & $\mathrm{Ib}$ \\
\hline Douillard 2000 & 387 & FOLFIRI vs. 5-FU/FS & 35 vs. $22(p<0,005)$ & 6,7 vs. $4,4(p<0,001)$ & $\begin{array}{l}17,4 \text { vs. } 14,1 \\
(p=0,031)\end{array}$ & Ib \\
\hline Saltz 2000 & 683 & IFL vs. Mayo (vs. CPT 11 mono) & 39 vs. $21(p<0,001)$ & 7,0 vs. $4,3(p=0,004)$ & 14,8 vs. $12,6(p=0,04)$ & $\mathrm{Ib}$ \\
\hline
\end{tabular}

Tab. 18 Oxaliplatin-versus irinotecanhaltige Protokolle in der Erstlinientherapie.

\begin{tabular}{|c|c|c|c|c|c|c|}
\hline Referenz & $\mathbf{n}$ & Therapieregime & Ansprechrate (\%) & PFS (Mo) & OS (Mo) & Evidenzlevel \\
\hline Tournigand 2004 & 220 & $\begin{array}{l}\text { FOLOFOX6 - FOLFIRI vs. rev } \\
\text { sequence }\end{array}$ & 54 vs. $56 n . s$ & 8,0 vs. 8,5 n.s & 20,6 vs. 21,5 n.s. & $\mathrm{lb}$ \\
\hline $\begin{array}{l}\text { Goldberg } 2004 \\
\text { [896] }\end{array}$ & 795 & FOLFOX vs. IFL (vs. IROX) & 45 vs. $31(p=0,002)$ (vs 35) & $\begin{array}{l}8,7 \text { vs. } 6,9(p=0,0014) \\
\text { (vs } 6,5)\end{array}$ & $\begin{array}{l}19,5 \text { vs. } 15(p=0,0001) \\
\text { (vs } 17,4)\end{array}$ & $\mathrm{Ib}$ \\
\hline $\begin{array}{l}\text { Goldberg } 2006 \\
\text { [897] }\end{array}$ & 305 & rIFL vs. FOLFOX & 32 vs. $48(p=0,006)$ & 5,5 vs. $9,7(p<0,0001)$ & 16,3 vs. $19(p=0,026)$ & $\mathrm{Ib}$ \\
\hline Colucci 2005 & 360 & FOLFIRI vs. FOLFOX & 31 vs. $34(p=0,6)$ & TTP: 7 vs. 7 & 14 vs. 15 & $\mathrm{Ib}$ \\
\hline $\begin{array}{l}\text { Comella } 2005 \\
\text { [898] }\end{array}$ & 274 & OXAFAFU vs. IRIFAFU & 44 vs. $31(p=0,029)$ & 7 vs. $5,8(p=0,046)$ & $\begin{array}{l}18,9 \text { vs. } 15,6 \\
(p=0,032)\end{array}$ & $\mathrm{Ib}$ \\
\hline
\end{tabular}

einen signifikanten Vorteil für die Kombinationstherapie, wobei die Entscheidung, ob nach dem AIO-Schema (wöchentlich) oder nach dem de Gramont-Schema (zweiwöchentlich) behandelt wurde, bei den einzelnen Zentren lag. Es wurden insgesamt 387 Patienten behandelt ( $\diamond$ Tab.17). Die Ansprechraten lagen bei $35 \%$ bei dem irinotecanhaltigen Protokoll und bei $22 \%$ bei der 5 FU/FS-Monotherapie $(\mathrm{p}<0,005)$. Das progressionsfreie Überleben und das Gesamtüberleben waren signifikant verlängert (6,7 vs. 4,4 Monate, $\mathrm{p}<0,001$ und 17,4 vs. 14,1 Monate, $\mathrm{p}=0,031$ ). Das Nebenwirkungsspektrum umfasste CTC-Grad-3- und -4-Diarrhöen in $44,4 \%$ (vs. 25,6\% im 5-FU-Monotherapiearm, $\mathrm{p}=0,055$ ) und Grad-3- und -4-Neutropenien in $28,8 \%$ der Patienten in der Irinotecangruppe (versus 2,4\% im 5-FU-Monotherapiearm, p =0,001) [891]. In einer weiteren Studie, in der 430 Patienten mit Irinotecan und einem wöchentlichen 5-FU-Schema (AIOSchema) in zwei unterschiedlichen Dosisstufen behandelt wurden, ergab sich im Vergleich mit dem alleinigen AIO-Schema ebenfalls ein Vorteil für die Kombinationstherapie. Die Unterschiede in den Ansprechraten und im progressionsfreien Überleben waren hochsignifikant, das Gesamtüberleben war jedoch nicht signifikant verlängert [892]. Die Grad-3- und -4-Toxizitäten in dieser Studie waren vor allem Diarrhöen (29\% bei irinotecanhaltigen Protokollen vs. $21 \%$ bei 5-FU-Monotherapie). Saltz zeigte in seiner dreiarmigen Studie, dass die Kombinationstherapie aus Bolus 5-FU und Irinotecan (IFL) ebenfalls in deutlich verbesserten Ansprechraten und einer signifikanten Verlängerung des progressionsfreien Überlebens und des Gesamtüberlebens im Vergleich mit einer Monotherapie aus Bolus 5-FU/FS oder Irinotecan resultiert, die Ergebnisse für die Irinotecan-Monotherapie und Bolus 5-FU-Monotherapie waren vergleichbar. Allerdings zeigte der Bolus 5-FU-Arm die höchste Rate an Grad-3- bis -4Neutropenien (66,2 vs. $53,8 \%$ bei Kombinationstherapie und $31,4 \%$ nach Irinotecanmonotherapie) und neutropenen Komplikationen mit 14,6\% (vs. 7,1 resp. 5,8\%). Die gastrointestinale Toxizität mit Diarrhö und Erbrechen war im Kombinationsarm führend [893]. Betrachtet man die unterschiedlichen Kombinationen aus 5-FU/FS und Irinotecan so sind FUFIRI und FOLFIRI (infusionales 5-FU) in ihrer Wirksamkeit etwa gleichwertig, wobei wahrscheinlich die Alopezierate des wöchentlichen Protokolls geringer ist. Irinotecan plus Bolus 5-FU (Saltz-Protokoll) weist das ungünstigste Verhältnis von Wirkung und Nebenwirkung auf und sollte daher keine Anwendung mehr finden.

\section{Vergleich Irinotecan- versus Oxaliplatinkombinationstherapien Hintergrund}

Besteht die Indikation für eine Kombinationschemotherapie, so kann FOLFOX oder FOLFIRI in der Erstlinientherapie eingesetzt werden.

Obwohl bei zwei der drei irinotecanhaltigen Kombinationschemotherapien im Gegensatz zu den oxaliplatinbasierten Protokollen ein Überlebensvorteil gezeigt werden konnte (s.o.), sind FOLFOX und FOLFIRI im direkten Vergleich hinsichtlich ihrer Wirksamkeit als gleichwertig zu betrachten [894]. Daher sollte bei der Wahl für eine fluoropyrimdinbasierte irinotecan- oder oxaliplatinhaltige Kombination das Toxizitätsspektrum besondere Beachtung finden. Colucci und Mitarbeiter dokumentierten im direkten Vergleich von FOLFIRI mit FOLFOX bei gleicher Wirksamkeit (s. u.) unterschiedliche Toxizitäten. Am häufigsten waren Nebenwirkungen im Gastrointestinaltrakt und Alopezie in Arm A (Irinotecan) und Thrombozytopenie und Neuropathien in Arm B (Oxaliplatin). Grad-3- und -4-Toxizitäten wurden in beiden Armen beobachtet, so die Neutropenie (27 bzw. 28\%) und Diarrhöen $(28 \%)$ vor allem bei den irinotecan- und Neuropathie (12\%) bei den oxaliplatinhaltigen Protokollen [895] ( $\bullet$ Tab. 18).

\section{Capecitabin in Kombination mit Oxaliplatin oder Irinotecan Oxaliplatin und Capecitabin Hintergrund}

Es liegen zwei große Phase-III-Studien mit insgesamt 822 Patienten vor, die infusionales 5-FU/FS mit der oralen Vorstufe Capecitabin jeweils in Kombination mit Oxaliplatin verglichen. Die spanische Arbeitsgruppe wählte das FUOX-Regime als Standardarm, in der Arbeit der AIO-Studiengruppe wurde ein infusionales wöchentliches 5-FU/FS/Oxaliplatin-Protokoll (FUFOX) eingesetzt $[899,900]$. In der deutschen Studie wurde im CAPOX-Arm ein progressionsfreies Überleben von 7,1 Monaten (vs. 8 Monate im FUFOX-Arm; HR: 1,17; 95 \% CI: 0,96-1,43; p =0,117) und ein Gesamtüberleben von 16,8 Monaten (vs. 18,8 Monate im FUFOXArm; HR: 1,12; $95 \%$ CI: 0,92-1,38; $\mathrm{p}=0,26$ ) dokumentiert. Die Ansprechraten lagen bei $48 \%$ für CAPOX (95\% CI: $41-54 \%$ ) 
Tab. 19 Capecitabin und Oxaliplatin-Kombinationen in der Erstlinientherapie - Phase-III-Studien.

\begin{tabular}{|c|c|c|c|c|c|c|}
\hline Referenz & $\mathbf{n}$ & Therapieregime & Ansprechrate (\%) & PFS (Mo) & OS (Mo) & Evidenzlevel \\
\hline $\begin{array}{l}\text { Cassidy 2008NO } \\
16966\end{array}$ & 2034 & XELOX vs. FOLFOX4 & 37 vs. 37 & $\begin{array}{l}8 \text { vs. } 8,5 \text { (HR: } 1,04 ; 97,5 \% \text { Cl: } \\
0,93-1,16)\end{array}$ & $\begin{array}{l}19,8 \text { vs. } 19,6 \text { (HR: } 0,99 ; 97,5 \% \\
\text { Cl: } 0,88-1,12) ; p=\text { n. s. }\end{array}$ & $\mathrm{Ib}$ \\
\hline Porschen 2007 & 474 & CAPOX vs. FUFOX & 48 vs. 54 & $\begin{array}{l}7,1 \text { vs. } 8,0 \text { (HR: } 1,17 ; 95 \% \mathrm{Cl}: \\
0,96-1,43 ; p=0,117)\end{array}$ & $\begin{array}{l}16,8 \text { vs. } 18,8 \text { (FUFOX HR: } 1,12 \\
95 \% \mathrm{Cl}: 0,92-1,38 ; \mathrm{p}=0,26 \text { ) }\end{array}$ & $\mathrm{lb}$ \\
\hline Diaz-Rubio 2007 & 348 & CAPOX vs. FUOX & 37 vs. 46 , n.s. & 8,9 vs. $9,5(p=0,153)$ & 18,1 vs. $20,8(p=0,145)$ & $\mathrm{Ib}$ \\
\hline
\end{tabular}

Tab. 20 Capecitabin in Kombination mit Irinotecan in der Erstlinientherapie - Phase-III-Studien.

\begin{tabular}{|c|c|c|c|c|c|c|}
\hline Referenz & $n=$ & Therapieregime & Ansprechrate (\%) & PFS (Mo) & OS (Mo) & Evidenzlevel \\
\hline $\begin{array}{l}\text { Koopman } 2007 \\
\text { (CAIRO) }\end{array}$ & 820 & CAPIRI vs. Cape mono & 41 vs. $20(p<0,0001)$ & 7,8 vs. $5,8(p=0,0002)$ & $\begin{array}{l}17,4 \text { vs. } 16,3 \\
\text { n. s. }\end{array}$ & $\mathrm{Ib}$ \\
\hline $\begin{array}{l}\text { Fuchs } 2007 \\
\text { (BICC-C) }\end{array}$ & 430 & $\begin{array}{l}\text { FOLFIRI vs. mIFL vs. } \\
\text { CAPIRI }\end{array}$ & 47 vs. 43 vs. 39 n. s. & $\begin{array}{l}7,6 \text { vs. } 5,9 \text { vs. } 5,8(p=0,004 \\
\text { und } 0,015 \text { resp.) }\end{array}$ & 23,1 vs. 17,6 vs. 18,9 n. s. & $\mathrm{Ib}$ \\
\hline $\begin{array}{l}\text { Kohne } 2007 \\
\text { (EORTC } 40 \text { 015) }\end{array}$ & 85 & $\begin{array}{l}\text { CAPIRI vs. FOLFIRI } \\
( \pm \text { Celecoxib) }\end{array}$ & 48 vs. 46 & 5,9 vs. 9,6 & 14,8 vs. 19,9 & IIb \\
\hline
\end{tabular}

resp. $54 \%$ für das FUFOX-Regime (95\% CI: 47 - $60 \%$ ). Die häufigste nicht hämatologische Nebenwirkung war eine Polyneuropathie in 27 resp. $25 \%$ der Fälle, nur das Hand-Fuß-Syndrom 2. und 3. Grades trat im CAPOX-Arm signifikant häufiger auf $(p=0,028)$ [900].

Studiendesign und Ergebnisse waren in beiden Studien vergleichbar, beide Therapieregime stellen somit eine aktive Erstlinientherapie dar.

Eine weitere große Phase-III-Studie zeigte klar die Nichtunterlegenheit von Capecitabin und Oxaliplatin (im XELOX-Protokoll) bezüglich des progressionsfreien und des Gesamtüberlebens im Vergleich mit FOLFOX4 (HR: 1,04; 97,5\% CI: 0,93-1,16 für PFS und HR: 0,99; 97,5\% CI: 0,88-1,12 für OS) [901] (৫ Tab. 19).

\section{Irinotecan und Capecitabin \\ Hintergrund}

Die Datenlage zu Wirksamkeit und Toxizität von Capecitabin und Irinotecan ist weniger einheitlich als bei der Kombination Capecitabin/Oxaliplatin. Zwei Arbeiten untersuchten die Wirksamkeit von CAPIRI im Vergleich zu einem infusionalen 5-FU-Protokoll. In der BICC-C Studie war CAPIRI dem FOLFIRI-Protokoll im progressionsfreien Überleben klar unterlegen. Wahrscheinlich spielt hier die sehr hohe Toxizität mit fast $50 \%$ Grad-3- bis -4-Diarrhöen eine entscheidende Rolle $\left(2 \times 1 \mathrm{~g} / \mathrm{m}^{2}\right.$ Capecitabin/Tag 1-14, $250 \mathrm{mg}$ Irinotecan $/ \mathrm{m}^{2}$ an Tag 1) Die Ansprechraten und das Gesamtüberleben waren nicht signifikant unterschiedlich [902]. Eine 2007 publizierte Arbeit berichtet von intolerablen Nebenwirkungen des CAPIRI-Regimes, die auch zum vorzeitigen Abbruch der Studie führten $\left(2 \times 1 \mathrm{~g} / \mathrm{m}^{2}\right.$ Capecitabin/Tag $1-15$ und 22 - 36, $250 \mathrm{mg}$ Irinotecan/m² Tag 1+22) In der CAPIRI Gruppe war vor allem aufgrund hämatotoxischer Nebenwirkungen in $61 \%$ der Fälle eine Dosisreduktion erforderlich, im FOLFIRI-Arm waren es nur $7 \%$. Sechs von acht Todesfällen traten im mit CAPIRI behandelten Kollektiv auf, drei Patienten starben an einem thromboembolischen Ereignis und drei infolge schwergradiger Diarrhöen [903]. Die CAIRO-Studie ist mit 820 Patienten die bisher größte Studie, die sich mit der Kombination von Capecitabin und Irinotecan befasste. In dieser Studie wurde Capecitabin in einer Dosis von $2 \times 1 \mathrm{~g} / \mathrm{m}^{2} /$ Tag 1 - 14 zusammen mit Irinotecan $250 \mathrm{mg} / \mathrm{m}^{2} /$ Tag 1 in einem 3-Wochenzyklus verabreicht. Die Häufigkeit von Grad-3- bis -4-Toxizitäten unterschied sich, mit
Ausnahme des häufigeren Auftretens eines Grad-3-Hand-FußSyndroms bei der sequenziellen Behandlung, nicht signifikant zwischen den beiden Gruppen [868].

In dieser Studie war die Rate an schwerwiegenden Diarrhöen deutlich niedriger als in der BICC- und der EORTC-Studie, was möglicherweise mit der Tatsache zusammenhängt, dass die holländischen Zentren der CAIRO-Studie gezielt für die Studiendurchführung und das Management möglicher Nebenwirkungen trainiert wurden. Das CAPIRI-Regime kann zum jetzigen Zeitpunkt aufgrund der Toxizität nicht als Standardbehandlung angesehen werden. Vorläufige Daten der AIO deuten jedoch darauf hin, dass CAPIRI in dosisreduzierter Form $\left(200 \mathrm{mg} / \mathrm{m}^{2}\right.$ Irinotecan Tag $1+2 \times 800 \mathrm{mg} / \mathrm{m}^{2}$ Capecitabin/Tag $\left.1-14\right)$ mit vertretbaren Nebenwirkungen einhergeht. Die häufigsten CTC-Grad-3- bis -4Toxizitäten waren Diarrhöen 17/15,5\%, Hand-Fuß-Syndrom in $5,9 / 2,7 \%$ und Neurotoxizität in $15,3 / 0 \%$ aller Patienten [904] (० Tab. 20).

\section{Irinotecan, Oxaliplatin + 5-FU als Kombinationstherapie - FOLFOXIRI}

\section{Hintergrund}

In einer Phase-III-Studie, die 2006 veröffentlicht wurde, konnte keine statistische Überlegenheit des FOLFOXIRI-Regimes im Vergleich mit FOLFIRI gezeigt werden. Das mediane Gesamtüberleben lag bei 21,5 vs. 19,5 Monaten, die Ansprechraten bei 43 vs. $33,6 \%(p=0,337$ resp. $p=0,168$ ). Die Rate an Nebenwirkungen (Alopezie, Diarrhö, Neurotoxizität) war signifikant höher als im FOLFIRI-Arm ( $p=0,0001$ resp. $p=0,001$ für Neurotoxizität) [863]. Falcone et al konnten dagegen mit einer höheren Dosierung signifikante Verbesserungen sowohl im progressionsfreien als auch im Gesamtüberleben nachweisen. Dieser Überlebensvorteil wurde durch eine signifikant häufiger auftretende Grad 2-3 periphere Neurotoxizität und Grad-3- bis -4-Neutropenien erkauft ( 0 vs. $19 \%$ resp. 28 vs. $50 \%, p<0,001$ resp. $p=0,0006$ ) [859]. Die Ansprechraten waren in beiden Arbeiten im Vergleich besser als mit FOLFIRI alleine, bei Falcone erreichte dieser Vorteil Signifikanzniveau. Die Ergebnisse der beiden Studien sind jedoch aufgrund unterschiedlicher Protokolle und Studienpopulation nur bedingt vergleichbar. Die Ansprechraten und Resektionsraten als primärer und sekundärer Endpunkt der italienischen Arbeit sind überzeugend, sodass das FOLFOXIRI-Protokoll v.a. zur Remissionsinduktion und möglicher sekundärer Resektion von Leber- 
Tab. 21 FOLFOXIRI in der Erstlinientherapie.

\begin{tabular}{|c|c|c|c|c|c|c|}
\hline Referenz & n & Therapieregime & Ansprechraten (\%) & PFS (Mo) & OS (Mo) & Evidenzlevel \\
\hline Falcone $2007^{1}$ & 244 & FOLFOXIRI vs. FOLFIRI & 60 vs. $34(p=0,0001)$ & $\begin{array}{l}9,8 \text { vs. } 6,9 \\
(p=0,0006)\end{array}$ & $\begin{array}{l}22,6 \text { vs. } 16,7 \\
(p=0,032)\end{array}$ & $\mathrm{lb}$ \\
\hline Souglakos $2006^{2}$ & 283 & FOLFOXIRI vs. FOLFIRI & 43 vs. $33,6(p=0,168)$ & 8,4 vs. $6,5(p=0,17)$ & $\begin{array}{l}21,5 \text { vs. } 19,5 \\
(p=0,337)\end{array}$ & $\mathrm{Ib}$ \\
\hline
\end{tabular}

15 -FU als kontinuierliche Infusion.

2 5-FU als Bolus-Protokoll mit reduzierten Dosen von Irinotecan und Oxaliplatin.

metastasen in Erwägung gezogen werden sollte (s. auch VII.2.1.2) (৫ Tab.21).

\section{5-FU/Folinsäure + Bevacizumab \\ Hintergrund}

Diese Kombination ist v. a. geeignet für Patienten die für ein oxaliplatinhaltiges/irinotecanhaltiges Protokoll nicht qualifizieren, oder Patienten, bei denen als Therapieziel eine Verlängerung des progressionsfreien und des Gesamtüberlebens bei guter Lebensqualität im Vordergrund steht (entsprechend Gruppe 3). In einer prospektiv, randomisierten placebokontrollierten Phase-II-Studie untersuchten Kabinavaar et al. die Wirksamkeit einer Kombination von Bevacizumab mit einer 5-FU/FS-Monotherapie nach dem Roswell-Park-Schema im Vergleich mit einer reinen FU/FSMonotherapie [905]. Die Ergebnisse zeigen eine hochsignifikante Verlängerung des progressionsfreien Überlebens in der Kombination mit Bevacizumab (9,2 vs. 5,5 Monate, $p=0,0002$ ), ohne dass eine signifikante Verbesserung der Ansprechraten erzielt werden konnte ( 26 vs. 15,2\%, p =0,055). Der Unterschied im Gesamtüberleben war ebenfalls nicht signifikant (16,6 vs. 12,9 Monate, $\mathrm{p}=0,16$ ). Obwohl es sich bei den Patienten dieser Studie um eine Hochrisiko-Population handelte (das mediane Alter lag bei 72 Jahren, der Performance Status war bei $72 \%$ der Patienten $>0$ ), wurde die Therapie gut vertragen.

\section{Oxaliplatin + 5-FU/Folinsäure + Bevacizumab \\ Hintergrund}

Die kürzlich publizierte multizentrische randomisierte Studie NO 16966 war initial als zweiarmige Studie konzipiert, die die Nichtunterlegenheit von Capecitabin und Oxaliplatin (als XELOX-Protokoll) im Vergleich mit FOLFOX4 untersuchen sollte [901]. Nach Publikation der Daten der Phase-III-Studie von Hurwitz (s. u.), die einen signifikanten Vorteil durch Hinzunahme von Bevacizumab ergab, wurde das ursprüngliche Studienprotokoll auf ein $2 \times 2$ faktorielles Design erweitert und um Bevacizumab ergänzt. Die Studie zeigte eine signifikante Verlängerung des progressionsfreien Überlebens in der Kombination aus Chemotherapie und Bevacizumab (XELOX+FOLFOX \pm Bevacizumab). Das PFS als primärer Endpunkt wurde im Median von 8,0 auf 9,4 Monate verlängert (HR: 0,83; 97,5\% CI: 0,72-0,95; p=0,0023) [864]. Eine mögliche Erklärung für diesen eher geringen Unterschied von nur 1,4 Monaten im PFS unter einer Dreifachkombinationstherapie könnte sein, dass die mittlere Behandlungsdauer in beiden Armen nur ca. 6 Monate (190 Tage) betrug, danach wurden Kombinationschemotherapie und Bevacizumab vermutlich aus Toxizitätsgründen ausgesetzt. So wurden nur $29 \%$ der mit Bevacizumab behandelten Patienten bis zum Progress behandelt. Eine geplante Subgruppenanalyse dieser Patienten deutet darauf hin, dass die Fortführung der Therapie bis zum Progress in einer Verlängerung der „Zeit der Tumorkontrolle“ resultieren könnte. Das Gesamtüberleben als sekundärer Endpunkt war nicht signifikant verlängert (21,3 vs. 19,9 Monate, HR: 0,89; 97,5\% CI: 0,76 - 1,03; $\mathrm{p}=0,077$ ), die Ansprechraten waren gleich (38 vs. $38 \%$; OR 1,00; 97,5\% CI: 0,78 - 1,28; p=0,99). 59 Patienten (8,4\%) im Bevacizumab-Arm und 43 Patienten $(6,1 \%)$ im Placebo-Arm wurden in kurativer Absicht operiert, der Effekt auf die R0-Resektabilität ist noch nicht sicher zu beurteilen. Resektabilität war in dieser Arbeit nicht als sekundärer Endpunkt definiert [864]. (s. auch 9.2.1.2).

\section{Irinotecan + 5-FU/Folinsäure + Bevacizumab \\ Hintergrund}

Der direkte Vergleich von IFL plus Placebo mit IFL plus Bevacizumab zeigte eine signifikante Verlängerung des Gesamtüberlebens von 15,6 Monaten auf 20,3 Monate ( $\mathrm{p}<0,001)$. Die mediane Zeit bis zum Progress konnte durch die Kombination mit dem Antikörper ebenfalls von 6,2 Monaten auf 10,6 Monate verlängert werden ( $p<0,001$ ). Die Ansprechraten waren mit 44,8\% in der Verumgruppe signifikant höher als in der Placebogruppe. (34,8\%; $\mathrm{p}=0,004)$. Das Toxizitätsspektrum umfasste eine Grad-3-Hypertonie bei $11 \%$ der im experimentellen Arm Behandelten im Gegensatz zu 2,3\% im Standardarm. Weitere Grad-3- und -4-Toxizitäten waren Leukopenie in $37 \%$ und Diarrhöen in $32,4 \%$. Bevacizumab war seitdem in Kombination mit 5-FU/FS mit oder ohne Irinotecan für die Erstlinientherapie des kolorektalen Karzinoms zugelassen [906]. Ein direkter Vergleich von FOLFIRI plus Bevacizumab mit mIFL plus Bevacizumab in der BICC-C Studie zeigte für das infusionale 5-FU-Protokoll eine klare Überlegenheit im progressionsfreien Überleben, Gesamtüberleben (noch nicht erreicht vs. 19,2 Monate mit mIFL + Bevacizumab, $p=0,007$ ). Die Ansprechraten waren nicht signifikant unterschiedlich (57,9 vs. $53,3 \%$ ) [902]. Auch aufgrund des günstigeren Toxizitätsprofils sollte dem infusionalen 5-FU-Protokoll der Vorzug vor IFL gegeben werden.

Oxaliplatin + Capecitabin + Bevacizumab

\section{Hintergrund}

Die Ergebnisse der N016966 Studie sind oben ausführlich dargelegt. XELOX ist sowohl in der Kombination mit Bevacizumab als auch ohne Antikörper dem infusionalen Protokoll nicht unterlegen. Die Ansprechraten, die von einem unabhängigen Review Komitee erhoben wurden sind gleich und liegen im FOLFOX und im XELOX-Arm bei jeweils $38 \%$. Eine prädefinierte Subgruppenanalyse deutet auf einen Vorteil von 1,9 Monaten im medianen progressionsfreien Überleben für die Kombination von Bevacizumab mit XELOX verglichen mit XELOX alleine hin (7,4 vs. 9,3 Monate, HR: 0,77; 97,5\% CI: 0,63-0,94; p=0,0026). Die Verfügbarkeit von Bevacizumab im FOLFOX-Arm resultierte in dieser Subgruppenanalyse in keiner signifikanten Verlängerung des progressionsfreien Überlebens (8,6 vs. 9,4, HR: 0,89; 97,5\% CI: 0,73-1,08; $\mathrm{p}=0,1871$ ) [864]. 


\section{5-FU-Folinsäure + Oxaliplatin + Cetuximab}

\section{Hintergrund}

Eine multizentrische randomisierte Phase-II-Studie aus Europa untersuchte die Wirksamkeit einer Kombination aus FOLFOX \pm Cetuximab (OPUS - Studie). 337 Patienten wurden randomisiert. Im experimentellen Arm waren die Ansprechraten 45,6\%, im Standardarm 35,7\% bei vergleichbarer Grad-3- bis -4-Toxizität. Überlebensdaten waren zum Zeitpunkt der Präsentation 2007 noch nicht erhältlich. Die häufigsten Nebenwirkungen waren Neutropenie, Diarrhö in beiden Gruppen und akneiformer Hautausschlag bei Behandlung mit Cetuximab [907]*.

Es gibt keine Phase-III-Daten, die eine Behandlung des mKRK mit einer Kombination von FOLFOX und Cetuximab in der Erstlinientherapie stützen.

Eine generelle Empfehlung zum Einsatz dieser Kombination kann deshalb derzeit nicht gegeben werden.

Cetuximab wird jedoch auch in Kombination mit Oxaliplatin bei Patienten mit einem k-ras Wildtyp exprimierendem Tumor für die Erstlinientherapie des KRK zugelassen.

\section{5-FU-Folinsäure + Irinotecan + Cetuximab}

\section{Hintergrund}

Für Cetuximab gibt es für die Erstlinientherapie noch keine als vollständiges Manuskript veröffentlichten Phase-III-Daten. Die Daten der im Jahr 2007 ASCO vorgestellten Phase-III-Studie (CRYSTAL) sind vielversprechend und weisen eine statistisch signifikante Erhöhung der Ansprechraten und des progressionsfreien Überlebens im Vergleich mit FOLFIRI alleine nach. Mit der Kombination von FOLFIRI und Cetuximab wurde eine Responserate von 46,9\% erreicht (im Vergleich mit 38,7\% bei FOLFIRI; $\mathrm{p}=0,005)$ Auch der Anteil an R0-Resektionen als sekundärer Endpunkt war im experimentellen Arm erhöht [860]*. Die hohen Ansprechraten aus Phase I/II Studien (ORR 67\%), die eine herausragende Rolle von Cetuximab als mögliche Therapie zum Downsizing marginal resektabler Lebermetastasen diskutiert hatten [908], wurden nicht erreicht.

Eine aktuell auf der ASCO vorgestellte retrospektive Analyse der CRYSTAL-Studie zeigte, dass ausschließlich Patienten mit Wildtyp k-ras exprimierenden Tumoren von einer Therapie mit Cetuximab profitieren [861]*.

Cetuximab kann entsprechend der Zulassung, die für August 2008 erwartet wird, bei diesem Patientenkollektiv in Kombination mit einer irinotecanhaltigen Therapie in der Erstlinienbehandlung des KRK eingesetzt werden.

\subsubsection{Therapiedauer/Therapiepause in der Erstlinien- therapie-Reinduktion}

\subsection{Empfehlung (2008)}

Es gibt keine ausreichende Evidenz, die ein Absetzen einer einmal begonnenen medikamentösen Therapie bis zum erneuten Progress rechtfertigen würde.

Empfehlungsgrad B, Level of Evidence 1b, Konsens

\section{Hintergrund}

Da das Gesamtüberleben und die Dauer der Tumorkontrolle in jeder Therapielinie durch neuere Chemotherapeutika und Kombinationschemotherapien deutlich verlängert werden kann, wird auch die Frage der chemotherapieassoziierten Nebenwirkungen und der resultierenden Lebensqualität immer entscheidender. Daher wurden Studien aufgelegt, die den Nutzen einer Erhaltungstherapie mit weniger intensiven Therapieschemata (OPTIMOX 1) oder eine komplette Therapiepause nach einer Induk- tionsphase im Gegensatz zur Behandlung bis zum Progress prüften (OPTIMOX 2). Die OPTIMOX-1-Studie untersuchte die Gabe von FOLFOX4 bis zum Progress im Vergleich mit FOLFOX7 gefolgt von einer Erhaltungstherapie mit 5-FU/FS und Reinduktion von FOLFOX7 bei Progress. Die Unterschiede im progressionsfreien und medianen Überleben waren nicht signifikant, die Rate an Grad-3-Neurotoxizitäten war günstiger (13 vs. 18\%, $\mathrm{p}=0,12$ ) [909]. Eine solche Deeskalationsstrategie mit Reinduktion von Oxaliplatin bei dokumentiertem Progress sollte auch im klinischen Alltag Anwendung finden und als mögliche Option mit den Patienten besprochen werden. Die OPTIMOX-2-Studie prüfte die Möglichkeit einer kompletten Therapiepause im Vergleich mit einer Erhaltungstherapie wie in OPTIMOX 1. Das mediane progressionsfreie Überleben war bei diesem Studienkonzept im Kontrollarm (OPTIMOX 1) signifikant länger als im Prüfarm (8,3 Monate vs. 6,7 Monate; $p=0,04$ ), auch das mediane Gesamtüberleben war deutlich besser (24,6 Monate vs. 18,9 Monate; $\mathrm{p}=0,05$ ). Die Daten liegen bislang nur als Kongressbericht vor und waren in der Präsentation nicht vollständig konklusiv [910]*. Eine Empfehlung für eine geplante vollständige Therapiepause ohne Erhaltungstherapie kann auf Grundlage dieser Daten nicht ausgesprochen werden. Die Arbeitsgruppe um Maughan konnte bei deutlich geringerer Toxizität einer intermittierenden Gabe eines 5-FU-haltigen Protokolls keinen klaren Überlebensvorteil für die kontinuierliche Gabe feststellen (HR 0,87 für intermittierende Gabe, $95 \%$ CI: 0,69 - 1,09; P= 0,23) [911].

Labianca zeigte mit einem intermittierenden FOLFIRI-Protokoll im Vergleich mit einer kontinuierliche Gabe bis zum Progress keinen Unterschied im Gesamtüberleben als primärem Endpunkt bei vergleichbarer Toxizität ( $\mathrm{HR}=1,11 ; 95 \% \mathrm{Cl}: 0,83-1,48)$ [912]. Im Gegensatz zu einer konzeptionellen Therapiepause gibt es kurzfristige Unterbrechungen der Chemotherapie, die durch die persönliche Lebenssituation des Patienten (z.B. Urlaub) bedingt sind. In diesem Fall sind kurzfristige Therapiepausen vertretbar (o Tab. 22).

\subsubsection{Chemotherapieprotokolle in der Zweit- und}

Drittlinientherapie

Insgesamt weisen die Studien zur Erstlinientherapie des mKRK eine hohe Variabilität im Gesamtüberleben auf, die von 14,1 bis über 22 Monate reicht. Geht man davon aus, dass keine großen Unterschiede in der Patientenselektion vorlagen, ist die Art der gewählten Zweit- und Drittlinientherapie und sicher auch deren unterschiedliche Verfügbarkeit möglicherweise für diese diskrepanten Ergebnisse verantwortlich. Die Wahl der Zweit- und Drittlinientherapie hängt sowohl von vorangegangenen Therapien und der therapiefreien Zeit als auch von der individuellen Patientensituation und dem jeweiligen Therapieziel ab. Während in der Erstlinientherapie derzeit über Optionen der Verkürzung der Therapiedauer oder „Stop-and-go“-Strategien nachgedacht wird (s. o.), gilt für die Zweit- und Drittlinientherapien weiterhin der Grundsatz dass die Therapie bis zum Progress der Erkrankung durchgeführt werden soll.

\subsection{Empfehlung (2008)}

Aufgrund unzureichender Evidenz soll mit Ausnahme der Fluoropyrimidine oder der Gabe von Irinotecan in Kombination mit Cetuximab nach Versagen einer irinotecanhaltigen Therapie keines der oben beschriebenen Therapeutika nach dokumentiertem Progress unter Therapie weiter appliziert werden. Dies gilt auch für Cetuximab und Bevacizumab.

Empfehlungsgrad A, Level of Evidence 2, starker Konsens 
Tab. 22 Studien zur Frage der Therapiepause/Erhaltungstherapie.

\begin{tabular}{|c|c|c|c|c|c|c|}
\hline Referenz & $\mathbf{n}$ & Therapieregime & Ansprechraten (\%) & PFS (Mo) & OS (Mo) & Evidenzlevel \\
\hline Labianca 2006 & 336 & $\begin{array}{l}\text { FOLFIRI intermitt vs. } \\
\text { cont. }\end{array}$ & 29 vs. 35 & $\begin{array}{l}8,8 \text { vs. } 7,3 \text { (HR: } 1,00 \text {, } \\
95 \% \mathrm{Cl}: 0,74-1,36 \text { ) }\end{array}$ & $\begin{array}{l}\text { 16,9 vs. } 17,6 \text { (HR: } 1,11 \\
95 \% \mathrm{Cl}: 0,83-1,48)\end{array}$ & $\mathrm{Ib}$ \\
\hline Tournigand (OPTIMOX 1) & 620 & $\begin{array}{l}\text { Ctx bis PD vs. Erhaltung } \\
\text { mit } 5 \text { FU/FS }\end{array}$ & 58,8 vs. 59,2 n. s. & 9 vs. 8,7 n.s. & 19,3 vs. 21,2 n. s. & Ib \\
\hline $\begin{array}{l}\text { Maindrault } 2007 \\
\text { (OPTIMOX 2) }\end{array}$ & 202 & Erhaltung vs. Pause & 63 vs. 61 & 8,3 vs. $6,7(p=0,04)$ & 24,6 vs. $18,9(p=0,05)$ & $\mathrm{Ib}$ \\
\hline Maughan 2003 & 354 & 5-FU/FS12w Ctx vs. cont. & 21 vs. $8 \%$ & $\begin{array}{l}3,7 \text { vs. } 4,9 \text { (HR: } 1,2, \\
95 \% \mathrm{Cl}: 0,96-1,49)\end{array}$ & $\begin{array}{l}10,8 \text { vs. } 11,3 \text { (HR: } 0,87 \\
95 \% \mathrm{Cl}: 0,69-1,09)\end{array}$ & IIb \\
\hline
\end{tabular}

Tab. 23 Oxaliplatinhaltige Protokolle in der Zweitlinientherapie.

\begin{tabular}{|c|c|c|c|c|c|}
\hline Referenz & $\mathrm{n}=$ & Therapieregime & Ansprechraten (\%) & PFS (Mo) & Evidenzlevel \\
\hline Giantonio 2007 [918] & 829 & $\begin{array}{l}\text { FOLFOX } 4 \text { vs. FOLFOX + Bevacizumab vs. } \\
\text { Bevacizumab mono }\end{array}$ & 8,6 vs. $22,7(p<0,0001)$ vs. 3,3 & $\begin{array}{l}4,7 \text { vs. } 7,3(p<0,0001) \text { vs. } \\
2,7\end{array}$ & Ib \\
\hline Rothenberg 2007 [919] & 627 & XELOX vs. FOLFOX & n. a. & TTP: 4,8 vs. 4,7 & $\mathrm{Ib}$ \\
\hline Tournigand 2004 & 220 & FOLFIRI $\rightarrow$ FOLFOX 6 vs. rev. Sequenz & 4 vs. 15 & 2,5 vs. 4,2 & Ib \\
\hline Rothenberg 2003 & 463 & $\begin{array}{l}\text { FOLFOX } 4 \text { vs. FS- } 5 \text { FU (vs. Oxaliplatin } \\
\text { mono) }\end{array}$ & 9,9 vs. 0 vs. 0 & TTP: 4,6 vs. $2,7(p<0,0001)$ & $\mathrm{Ib}$ \\
\hline
\end{tabular}

\section{Hintergrund}

Der Stellenwert einer effektiven Zweitlinientherapie für das Gesamtüberleben wurde in mehreren Phase-III-Studien untersucht. So erbrachte eine Zweitlinientherapie mit Irinotecan nach Versagen einer Fluorouracil-Monotherapie im Vergleich mit BSC [913] oder infusionalem 5-FU/FS [914] einen deutlichen Vorteil im Gesamtüberleben.

Eine Kombinationstherapie mit Oxaliplatin und Fluororuracil nach Versagen eines irinotecanhaltigen Protokolls in der Erstlinientherapie war einer 5-FU/FS bzw. Oxaliplatin-Monotherapie sowohl bezüglich der erzielten Ansprechraten als auch bezüglich der Zeit bis zur Progression überlegen [915]. Die Kombinationstherapie von 5-FU/FS mit Oxaliplatin bzw. Irinotecan, die als Erstlinientherapie jeweils Ansprechraten (CR + PR) von 40 - $55 \%$ zeigen, erreichen in der Zweitlinientherapie Ansprechraten von 4\% (FOLFIRI) bis $15 \%$ (FOLFOX) und ein progressionsfreies Überleben von ungefähr 2,5 - 4,2 Monaten. Das mediane Überleben der Patienten beträgt für beide Therapiesequenzen (FOLFOX $\rightarrow$ FOLFIRI bzw. FOLFIRI $\rightarrow$ FOLFOX) jeweils etwa 20 Monate [894]. Durch die Einführung monoklonaler Antikörper wie Cetuximab, Bevacizumab und Panitumumab, haben sich die Therapiemöglichkeiten auch in der Zweitlinientherapie erweitert. Die sog. BOND 1-Studie belegte erstmals die Effektivität von Cetuximab in Kombination mit Irinotecan in der Zweitlinientherapie des fortgeschrittenen KRK nach Irinotecanversagen (RR 22,9\%, Gesamtüberleben 8,6 Monate) [916]. Die sog. EPIC-Studie konnte zeigen, dass die Kombination Cetuximab plus Irinotecan auch nach einer oxaliplatinhaltigen Vortherapie wirksam und im direkten Vergleich wirksamer ist als eine Irinotecan-Monotherapie [917].

\subsubsection{Kombinationstherapie in der Zweit - und}

Drittlinientherapie

\section{Hintergrund}

In seiner 2004 publizierten Arbeit verglich Tournigand FOLFOX und FOLFIRI jeweils als Erst- resp. Zweitlinientherapie und vice versa. Das mediane Überleben der jeweiligen Sequenz unterschied sich nicht signifikant zwischen den beiden Armen (20,6 Monate für FOLFOX vs. 21,5 Monate für FOLFIRI in der Erstbe- handlung) [894]. Auch bezüglich der Ansprechraten oder des progressionsfreien Überlebens konnte für keine Sequenz ein signifikanter Vorteil gezeigt werden ( $\bullet$ Tab. 23).

\section{Oxaliplatin + Fluoropyrimidin ( $\bullet$ Tab. 23)}

Capecitabin und Oxaliplatin

\section{Hintergrund}

627 Patienten mit Progress unter einer irinotecanhaltigen Therapie wurden in der zweiten Linie entweder mit XELOX oder mit FOLFOX behandelt. Die mittlere Zeit bis zum Progress lag im XELOX-Arm bei 4,8 Monaten gegenüber 4,7 Monaten im FOLFOXArm.

Grad-3/4-Toxizitäten traten in 60,1\% der Fälle im XELOX-Arm und bei 72,4\% der mit FOLFOX behandelten Patienten auf. Es handelte sich hauptsächlich um Diarrhöen (20 vs. $5 \%$ ), Neutropenie in 5 resp. 35\% und Übelkeit und Erbrechen in 5-6\% der Fälle [919].

\section{Irinotecan + Cetuximab}

\section{Hintergrund}

In der BOND-Studie wurden 329 Patienten behandelt, die innerhalb von drei Monaten nach irinotecanhaltiger Therapie progredient waren. Die Patienten wurden entweder für eine Chemotherapie mit Irinotecan und Cetuximab oder eine CetuximabMonotherapie randomisiert. Mit der Kombinationstherapie konnten, verglichen mit denen der Monotherapie, signifikant höhere Ansprechraten erzielt werden (22,9 vs. $10,8 \%, p=0,007)$. Auch das progressionsfreie Überleben war signifikant verlängert. (4,1 vs. 1,5 Monate, $\mathrm{p}<0,001$ ) Das Gesamtüberleben lag bei 8,6 resp.6,9 Monaten $(p=0,48)[916]$.

Die EPIC-Studie untersuchte die Effektivität dieser Kombination bei Patienten, die unter einer oxaliplatinbasierten Therapie progredient waren. In dieser Phase-III-Studie wurden die Patienten entweder mit Cetuximab + Irinotecan $(n=648)$ oder mit Irinotecan mono $(n=650)$ behandelt. Die Ansprechraten waren bei Erhalt einer Kombinationstherapie signifikant verbessert (16,4 vs. $4,2 \% ; \mathrm{p}<0,0001)$. Das mediane PFS verlängerte sich mit der Verfügbarkeit von Cetuximab von 2,6 Monate auf 4,0 Monate (HR: 
0,692, p<0,0001). Das mediane Gesamtüberleben als primärer Endpunkt war in beiden Armen vergleichbar, da nach Progression ein „crossover“ in den anderen Studienarm gestattet war (10,7 vs. 10 Monate, Hazard Ratio für das Gesamtüberleben $=0,975,95 \% \mathrm{CI}: 0,854-1,114, \mathrm{p}=0,71)$. Eine begleitende Lebensqualitätsanalyse ergab eine Verbesserung für den allgemeinen Gesundheitsstatus $(p=0,047)$ und für funktionelle und individuelle Symptome (Fatigue, Nausea/Vomitus $(p<0,0001)$, Schmerz $(\mathrm{p}<0,0001)$ im Kombinationsarm, sodass vorbehaltlich der Zulassung bei symptomatischen Patienten diese Kombination erwogen werden kann $[917,920]^{*}$.

\section{Bevacizumab + 5-FU-Folinsäure + Oxaliplatin \\ Hintergrund}

Der Stellenwert von Bevacizumab in Kombination mit Oxaliplatin und 5FU/FS in der Zweitlinientherapie nach Versagen einer irinotecanhaltigen Therapie bei nicht mit Bevacizumab vorbehandelten Patienten wurde in einer Phase-III-Studie untersucht. 829 Patienten wurden in einen der drei Therapiearme, FOLFOX-Bevacizumab, bzw. FOLFOX oder Bevacizumab jeweils als Monotherapie, randomisiert. Die Hinzunahme von Bevacizumab resultierte in einem signifikanten Überlebensvorteil von 2,1 Monaten im Vergleich mit FOLFOX alleine. (12,9 vs. 10,8 Monate, HR: 0,75; p=0,0011). Auch das progressionsfreie Überleben war signifikant länger als im alleinigen Chemotherapiearm (7,3 vs. 4,7 Monate, HR: 0,61, p <0,0001). Bevacizumab alleine hatte keinen klinischen Stellenwert. Die Kombination mit dem VEGF-Antikörper erhöhte die Rate an Grad-3und -4-Toxizitäten um 14\%. Im experimentellen Arm kam es signifikant häufiger zu Blutungen, Vomitus und Hypertonus. Das ebenfalls erhöhte Neuropathierisiko ist am ehesten mit der längeren Behandlungsdauer im Kombinationsarm (10 Zyklen vs. 7 Zyklen im FOLFOX-Arm) assoziiert. Der Nachbeobachtungszeitraum betrug 28 Monate [918].

\section{Mitomycin C + Fluoropyrimidin \\ Hintergrund}

In einer Arbeit von Chong mit 36 Patienten konnte in der Drittlinientherapie eine objektive Ansprechrate von 15,2\% erzielt werden. Das mediane Gesamtüberleben lag bei 9,3 Monaten. Die Daten aus Phase I/II Studien sind ermutigend, Ergebnisse aus PhaseIII-Studien liegen nicht vor. Fluoropyrimidin und Mitomycin C kann als Salvage-Therapie angewandt werden [921 - 924].

\subsubsection{Monotherapie mit biologischen Substanzen in der Drittlinienlinientherapie \\ Cetuximab}

\section{Hintergrund}

2006 publizierte Lenz eine große einarmige Phase-II-Studie mit 346 Patienten, die ebenfalls den Nutzen einer Cetuximab-Monotherapie bei irinotecanrefraktären Patienten mit einer Ansprechrate von $11,6 \%$ zeigte. Mehr als die Hälfte der Patienten war zum Zeitpunkt der ersten Bildgebung progredient (medianes progressionsfreies Überleben: 1,5 Monate), das Gesamtüberleben lag bei 6,6 Monaten. Die bereits erwähnte BOND-Studie zeigte vergleichbare Ansprechraten von ca. $10 \%$ in der Monotherapie. Auch in den letztgenannten Arbeiten korrelierte der Grad des Hautausschlags mit der Wirksamkeit [916, 925]. Eine ganz aktuell publizierte Arbeit, die eine Cetuximab-Monotherapie mit BSC verglich, erreichte ein Gesamtüberleben von 6,1 Monate gegenüber 4,6 Monaten in der Gruppe mit alleiniger BSC [926]. In Anbetracht dieser Daten und der Ergebnisse der BOND-1-Studie ist eine Cetuximab-Monotherapie v. a. bei Patienten zu empfehlen, die für eine irinotecanhaltige Therapie nicht qualifizieren. (Cetuximab kann wegen bislang fehlender Zulassung in der Monotherapie auch nur so eingesetzt werden).

Translationale Daten an einem heterogenen Patientenkollektiv weisen darauf hin, dass auch für Cetuximab der k-ras-Mutationsstatus einen unabhängigen prädiktiven Faktor darstellt. So fand sich eine k-ras-Mutation in $27 \%$ der irinotecanrefraktären Patienten und war sowohl mit einer Resistenz gegenüber Cetuximab assoziiert ( 0 vs. $40 \%$ Responder unter den 24 Patienten mit k-ras mutierten resp. den 65 Patienten mit kras-Wildtyp exprimierenden Tumoren $\mathrm{p}<0,001$ ) als auch mit einem schlechteren Überleben (medianes PFS: 10,1 vs. 31,4 Wochen bei Patienten ohne Mutation $\mathrm{p}=0,0001$; medianes OS: 10,1 vs. 14,3 Monate bei Patienten ohne Mutation; $\mathrm{p}=0,026$ ) [927].

Der k-ras-Mutationsstatus ist ab August 2008 zulassungsrelevant. Nur Patienten mit einem Wildtyp k-ras exprimierenden Tumor sollen Cetuximab erhalten.

\section{Panitumumab \\ Hintergrund}

Panitumumab ist der erste vollständig humane monoklonale Antikörper, der an den epidermalen Wachstumsfaktorrezeptor (EGFR) bindet. Eine Wirksamkeitsstudie der Phase II bei 148 Patienten mit vorbehandeltem metastasiertem kolorektalem Karzinom (Dosis Panitumumab 2,5 mg/kg/Woche) zeigte bei $9 \%$ ein partielles Ansprechen auf die Therapie, bei $29 \%$ blieb die Erkrankung stabil. Die mittlere Gesamtüberlebenszeit betrug 9 Monate, die mittlere Zeit bis zur Progression lag bei 14 Wochen [928]. Eine daraufhin durchgeführte multizentrische, randomisierte und kontrollierte Phase-III-Studie umfasste 463 Patienten mit metastasiertem kolorektalen Karzinom nach Versagen einer zytostatischen Standardtherapie mit 5-FU/FS, Irinotecan und Oxaliplatin. Die Patienten wurden randomisiert einem Behandlungsarm mit Panitumumab zusammen mit bester supportiver Behandlung $(n=231)$ oder alleiniger bester supportiver Behandlung $(\mathrm{n}=232)$ zugeteilt. Mit Panitumumab konnte eine signifikante Verbesserung des progressionsfreien Überlebens erreicht werden (HR: 0,54; $95 \%$ CI, 0,44-0,66, p<0,0001). Das mediane progressionsfreie Überleben lag bei 8 Wochen für Patienten, die mit Panitumumab behandelt wurden, im Gegensatz zu 7,3 Wochen bei alleiniger best supportive care. 176 der Patienten, die zuvor einer alleinigen besten supportiven Therapie zugeteilt worden waren, erhielten nach der Progression ihres Tumorleidens im cross-over Panitumumab [929]. Für 168 Patienten war der K-ras-Mutationsstatus erhältlich, 20 Patienten (12\%) der Patienten zeigten ein Ansprechen, bei 32\% blieb die Erkrankung stationär. Die Wirksamkeit von Panitumumab war auf Tumore beschränkt, die keine k-ras-Mutation aufwiesen. Diese tranlationalen Untersuchungen wurden aktuell publiziert [930]. In den USA wurde Panitumumab im September 2006, in Europa im Dezember 2007 zugelassen. Die Zulassung ist auf Patienten mit Tumoren beschränkt, die ein Wildtyp k-ras-Gen exprimieren und bei denen Fluoropyrimidin-, Oxaliplatin- und Irinotecanhaltige Chemotherapieregime versagt haben (Drittlinientherapie).

\subsection{Vorgehen beim Lokalrezidiv oder bei nicht} hepatischer und nicht pulmonaler Fernmetastasierung 9.5.1. Lokalrezidiv

Beim Rektumkarzinom-Rezidiv sollte, soweit noch keine Vorbehandlung (Radio(chemo)therapie) im Rahmen der Primäroperation erfolgte, diese nun primär favorisiert werden. Bei bereits vorbehandelten Patienten und der Möglichkeit einer R0-Resek- 
tion kann primär operiert werden [931]. Bei bereits vorbehandelten Patienten und fraglicher R0-Resektabilität eines Rezidivs muss individuell und unter Berücksichtigung der Intensität der Vortherapie bezüglich eines multimodalen chirurgischen Vorgehens entschieden werden.

Beim lokoregionären Lymphknotenrezidiv beim Kolonkarzinom sollte - sofern möglich - der Versuch einer kurativen kompletten Resektion erfolgen [932].

\subsubsection{Nicht hepatische oder nicht pulmonale Fernmetastasen}

\subsection{Empfehlung (2013)}

Bei Patienten mit einer isolierten und limitierten Peritonealkarzinose kann eine zytoreduktive Chirurgie gefolgt von einer hyperthermen intraperitonealen Chemotherapie (HIPEC) durchgeführt werden, wenn folgenden Voraussetzungen erfüllt sind:

- PCI (peritoneal cancer index) $<20$

- keine extraabdominellen Metastasen

- Möglichkeit der makroskopisch kompletten Entfernung oder Destruktion jeglicher Tumormanifestation

- Therapie in einem spezialisierten Zentrum

Die Durchführung im Rahmen von Studien sollte bevorzugt werden. Empfehlungsgrad 0, Level of Evidence 2a, starker Konsens

\section{Hintergrund}

Es existiert hierzu bisher nur eine einzige abgeschlossene randomisierte Studie zum Vergleich zwischen systemischer Chemotherapie und zytoreduktiver Chirurgie mit HIPEC [660]. Diese Studie konnte auch im Langzeitverlauf belegen, dass die chirurgische Therapie der Peritonealkarzinose zu signifikant besserem krankheits-spezifischem Überleben führt [661]. Ließ sich makroskopische Tumorfreiheit erzielen, lag das 5-Jahresüberleben bei $45 \%$. Einschränkend muss angeführt werden, dass der Kontrollarm lediglich eine 5-FU-basierte systemische Therapie erhielt. Trotz zahlreicher nicht randomisierter Studien mit ermutigenden Ergebnissen [662] ist damit nicht eindeutig definiert, welche Patienten über eine moderne systemische Polychemotherapie hinaus von einer chirurgischen Therapie mit HIPEC profitieren.

Bei isolierten Knochenmetastasen sollte bei Schmerzsymptomatik eine lokale Strahlentherapie eingeleitet werden. Hierbei zeigte sich in einer Metaanalyse die Einzelhochdosistherapie der fraktionierten Therapie äquipotent [933]. Die Einzelhochdosistherapie sollte daher im Rahmen des gesamtonkologischen Konzepts bevorzugt werden. Zusätzlich kann eine Biphosphonatgabe erfolgen. Bei Frakturgefährdung und drohender Querschnittlähmung ist eine Operation indiziert. Bei isolierten Hirnmetastasen sollte falls möglich eine operative Resektion erfolgen [934]. Bei Inoperabilität ist eine Strahlentherapie ggf. als stereotaktische Bestrahlung möglich [935].

\section{Nachsorge}

$\nabla$

Nach Diagnose und Therapie eines kolorektalen Karzinoms ist unabhängig vom Tumorstadium eine adäquate ärztliche Betreuung sinnvoll. Nach kurativer Therapie besteht für 5 Jahre ein erhöhtes Risiko für ein lokales oder lokoregionäres Rezidiv (3 $24 \%$ ), Fernmetastasen (25\%) oder einen metachronen Zweittumor (1,5-10\%) [936-945]. Das Risiko ist höher bei genetischer Veranlagung [940] und bei fortgeschrittenen Tumorstadien [946,
947]. Hieraus leitet sich grundsätzlich die Begründung für eine Nachsorge dieser Patienten ab: Ein Rezidiv sollte so früh entdeckt werden, dass ein operativer Zweiteingriff in kurativer Absicht möglich ist. Objektive Ziele sind neben der Heilung oder Lebensverlängerung bei Rezidiv, der Nachweis von Zweittumoren sowie der Nachweis und die Beseitigung nicht Rezidiv bedingter Folgen der Tumorkrankheit oder der operativen Therapie. Subjektive Ziele der Nachsorge sind auf die Verbesserung der Lebensqualität des Patienten ausgerichtet [948]

Ein zusätzliches Ziel stellt die Qualitätskontrolle der durchgeführten diagnostischen und therapeutischen Maßnahmen dar. Die Effektivität der Nachsorge scheint jedoch marginal mit im Mittel $1 \%$ verbessertem Überleben im Gesamtkollektiv der nachgesorgten Patienten [949]. Daten aus 267 Artikeln zu diesem Thema wurden in einer Metaanalyse ausgewertet [950]. Um einem Patienten mit kolorektalem Karzinom ein Langzeitüberleben zu ermöglichen, wurden 360 positive Nachsorgeteste und 11 Zweitoperationen benötigt. Die übrigen 359 Nachsorgemaßnahmen und 10 Operationen waren entweder ohne therapeutischen Gewinn oder mit negativen Auswirkungen verbunden [950].

In einem Cochrane Review von 2008 mit 8 eingeschlossenen Studien konnte gezeigt werden, dass eine intensive Nachsorge im Vergleich mit keiner oder einer minimalen Nachsorge mit einer geringeren Mortalität einhergeht (OR 0,73 [95\% CI 0,59-0,91]).

\subsection{Nachsorge bei Patienten im UICC-Stadium I \\ 10.1. Konsensbasierte Empfehlung (2013)}

Eine regelmäßige Nachsorge bei Patienten mit kolorektalem Karzinom und frühem Tumorstadium (UICC I) ist nach R0-Resektion in Anbetracht der geringen Rezidivrate und der günstigen Prognose nicht zu empfehlen. Eine rein koloskopische Nachsorge soll entsprechend Kapitel 10.3 erfolgen.

GCP

\section{Hintergrund}

Patienten im UICC-Stadium I haben eine gute Prognose nach kurativer Resektion. Dabei erleiden häufiger Patienten mit pT2 Tumoren (UICC Ib) (13\%) als mit pT1-Tumoren (UICC Ia) (4\%) ein Rezidiv [951]. Insgesamt ist das Langzeitüberleben im Stadium UICC I entsprechend dieser prospektiven Kohortenstudie mit $86 \%$ sehr gut und erlaubt einen weitgehenden Verzicht auf eine programmierte Nachsorge. Zu einem ähnlichen Ergebnis kam eine retrospektive Studie an 541 Patienten mit einem kolorektalen Karzinom im UICC-Stadium 1, wobei pT1NOM0 (UICC Ia) Tumoren 2,9\% Rezidive, und pT2 Karzinome (UICC Ib) 5,6\% Rezidive aufwiesen [952]. Im Stadium Dukes A wurden Rezidive nur bei Patienten mit Rektumkarzinom $(11 \%, n=6 / 55)$, nicht dagegen beim Kolonkarzinom beobachtet [953].

Abweichend kann im Einzelfall nach leitliniengerechter Therapie bei Annahme eines hohen lokalen Rezidivrisikos aufgrund des endoskopischen oder intraoperativen Befunds (z. B. nach intraoperativer Tumoreröffnung) oder eines pathologischen Befunds (z.B. erhöhtes Risiko für Fernmetastasen bei Invasion perikolischer Venen [954, 955], angiolymphatischer Invasion [956, 957], G3/G4-Tumoren oder pT2-Tumoren) eine engmaschigere Nachsorge angezeigt sein (vgl. Kapitel 8). Hierbei erwies sich die alleinige Bestimmung des CEA als ausreichend [952].

Da Patienten auch im Stadium I ein erhöhtes Risiko für die Entwicklung metachroner Zweittumoren besitzen, ist eine koloskopische Nachsorge entsprechend Kapitel 10.3 sinnvoll. 
10.2. Nachsorge bei Patienten im UICC-Stadium II und III 10.2. Empfehlung/konsensbasierte Empfehlung (2008)

Nach R0-Resektion von kolorektalen Karzinomen des UICC-Stadiums II und III sind regelmäßige Nachsorgeuntersuchungen (s. Empfehlung 10.1.) indiziert.

Empfehlungsgrad A, Level of Evidence 1a, [949, 950, 958 -967], starker Konsens

\subsection{Empfehlung (2008)}

Diese sollten jedoch nur durchgeführt werden, wenn bei einem Rezidiv therapeutische Konsequenzen zu erwarten sind. GCP starker Konsens

\section{Hintergrund}

Bei fortgeschrittenem kolorektalen Karzinomen (UICC-Stadium II und UICC-Stadium III) ist das Rezidivrisiko wesentlich höher [936 - 947]. Zum Nutzen von Nachsorgemaßnahmen bei diesem Tumor gibt es 6 randomisierte kontrollierte Studien [962 - 967], von denen nur 2 einen positiven Effekt auf das 5-Jahresüberleben einer intensivierten Nachsorge im Vergleich zu einer „Standardnachsorge“ nachweisen konnten [965, 967]. Fast alle Studien schlossen auch Patienten im UICC-Stadium I ein.

Verschiedene Metaanalysen von 5 randomisierten und kontrollierten Studien (1 positive, 4 negative) [949, 950, 958 - 961] ergaben einen geringen Überlebensvorteil für die Durchführung von mehr Maßnahmen in der Nachsorge gegenüber weniger Testen. Eine Bildgebung zur Beurteilung der Leber erwies sich ebenfalls in der Nachsorge als signifikant besser. Die Signifikanz ging jedoch verloren, wenn beide Ergebnisse nicht als Odds Ratio, sondern als Risikodifferenz berechnet wurden [960]. Eine aktive Nachsorge führte nur zu einem marginalen Überlebensvorteil nach 5 Jahren von 0,5 bis $2 \%$ [949].

Eine kürzliche Cochrane Publikation [968] schloss 3 weitere Studien in diese Metaanalyse ein [967, 969, 970]. Es fand sich ein Überlebensvorteil für intensiver nachgesorgte Patienten nach 5 Jahren (OR 0,73; 95\% CI 0,59-0,91), wobei aber die absolute Zahl an entdeckten Rezidiven in beiden Gruppen gleich war [30]. Eine retrospektive Studie untersuchte die Auswirkungen einer regelmäßigen Nachsorge entsprechend den ASCO-Leitlinien (> 70\% der Nachsorgetermine wurden wahrgenommen) mit geringer $(<70 \%)$ und keiner Nachsorge [971]. Bei regelmäßiger Nachsorge war im Vergleich zu den anderen Gruppen das 5 und 10-Jahresüberleben statistisch signifikant besser. Dies gilt auch für die Prognose dieser Patienten bei einem Rezidiv. Auch ein psychologischer Nutzen ist für die betroffenen Patienten aus Nachsorgemaßnahmen abzuleiten [972].

Leider wird den Leitlinien nicht immer gefolgt. So zeigte sich, dass bei Menschen jenseits des 65. Lebensjahres nur in 73,6\% der Fälle die empfohlenen Koloskopien und lediglich bei 46,7\% eine Bestimmung des CEA erfolgte, während nicht empfohlene Maßnahmen wie CT und PET-CT bei $48 \%$ bzw. 7\% durchgeführt wurden [973].

Aussagen zu Art und Häufigkeit der Nachsorgeuntersuchungen können nicht sicher gemacht werden, da hierzu gute Studien fehlen [974-976]. Eine Nachsorge adaptiert an die UICC Stadien oder die Auswirkungen eines kompletten Verzichts auf eine Nachsorge wurden bisher in keiner prospektiven Studie geprüft. Wegen der insgesamt unbefriedigenden Datenlage legte sich die Expertenkonferenz trotz eines Härtegrads 1a bei Vorliegen von mehreren Metaanalysen lediglich auf den Empfehlungsgrad B für das programmierte Vorgehen in der Nachsorge des kolorektalen Karzinoms des UICC-Stadiums II und III fest.

\subsection{Wertigkeit diagnostischer Methoden in der} Nachsorge

$\mathrm{Zu}$ den Inhalten der Nachsorge werden folgende Empfehlungen gegeben:

\subsubsection{Anamneseerhebung}

10.4. Konsensbasierte Empfehlung (2008)

Eine symptomorientierte Anamneseerhebung und körperliche Untersuchung sind grundsätzlicher Bestandteil der Nachsorge.

GCP, starker Konsens

\section{Hintergrund}

Anamnese und körperliche Untersuchung tragen wenig zur frühzeitigen Entdeckung eines Rezidivs bei kolorektalem Karzinom bei. Dennoch sollten diese ärztlichen Basismaßnahmen vor jeder weiteren Untersuchung erfolgen [975, 977]. Hierfür sprachen sich alle Teilnehmer der Konsensuskonferenz aus.

\subsubsection{CEA-Bestimmung}

\subsection{Empfehlung (2008)}

Die Bestimmung des karzinoembryonalen Antigens (CEA) wird alle 6 Monate für mindestens 2 Jahre empfohlen. Ein erhöhter CEA-Wert erfordert eine weitere Diagnostik, berechtigt aber nicht zum Beginn einer systemischen Chemotherapie bei Verdacht auf ein metastasiertes Tumorstadium.

Empfehlungsgrad B, Level of Evidence 4, [965, 978, 979], starker Konsens

\section{Hintergrund}

CEA erwies sich in der Nachsorge bei der frühzeitigen Entdeckung von Lebermetastasen besser als Koloskopie, Computertomografie und Sonografie [965, 978, 979]. Eine Metaanalyse von 7 nicht randomisierten Studien zeigte einen Überlebensvorteil von $9 \%$ für Patienten, bei denen im Nachsorgeprogramm CEA enthalten war [958]. Andere Studien führten zu keinem oder nur einem minimalen Nutzen $[963,980]$. CEA wird in der Nachsorge in einer Literaturübersicht nicht empfohlen [976]. Amerikanische (ASCO) und europäische (EGTM, European Group on Tumour Markers) Leitlinien zur Nachsorge enthalten jedoch das CEA [975, 977, 979], wobei die Bestimmung alle $2-3$ Monate in den ersten 2 Jahren empfohlen wird.

Die adjuvante Therapie mit 5-Fluoruracil kann zu falsch erhöhten Werten führen, sodass ein ausreichender Abstand zu dieser Behandlung eingehalten werden sollte [981]. 30\% aller kolorektalen Tumoren setzen CEA nicht frei [981, 982], während 44\% der Patienten mit normalen präoperativen Werten postoperativ einen Anstieg zeigen [983]. Bei der weiteren Abklärung des erhöhten CEA-Wertes sind bildgebende Verfahren und gegebenenfalls 18Fluorodeoxyglukose-Positronenemissionstomografie [984, 985] einzusetzen.

Wegen der insgesamt widersprüchlichen Daten für einen Einsatz von CEA in der Nachsorge kolorektaler Karzinome, wich die Expertenkonferenz von den ASCO- und EGTM-Empfehlungen ab und legte halbjährliche statt vierteljährliche Bestimmungsintervalle in den ersten 2 Jahren und danach jährlich über drei Jahre fest.

\subsubsection{Weitere Laborwerte}

10.6. Empfehlung (2008)

Die routinemäßige Bestimmung von Laborwerten im Rahmen der Nachsorge ist nicht zweckmäßig.

Empfehlungsgrad B, Level of Evidence 3b, [974, 977] starker Konsens 


\section{Hintergrund}

In zahlreichen Studien zur Nachsorge war die Bestimmung von Leberenzymen im Serum Bestandteil des Nachsorgeprogramms. Eine Studie zeigte jedoch, dass CEA und andere bildgebende Verfahren früher pathologisch ausfallen als die Leberfunktionsproben [974]. Aus diesem Grunde wird von einer routinemäßigen Bestimmung dieser Serumparameter abgeraten. Ähnliches gilt für die Bestimmung eines Blutbilds [977].

\subsubsection{FOBT}

10.7. Empfehlung (2008)

Die Untersuchung auf okkultes Blut im Stuhl ist für die Nachsorge nicht geeignet.

Empfehlungsgrad A, Level of Evidence 3b, [982, 986], starker Konsens

\section{Hintergrund}

Die Untersuchung auf okkultes Blut im Stuhl ist für die Nachsorge nicht geeignet. Nur $12 \%$ der lokalen Tumorrezidive führten zu einer oberflächlichen Verletzung der Schleimhaut [982]. Reihenuntersuchungen bei 1217 Patienten nach durchgemachtem kolorektalen Karzinom zeigten eine sehr niedrige Sensitivität und Spezifität dieses Testes für einen Rezidivtumor oder Polypen [986].

\subsubsection{Sonografie}

10.8. Empfehlung (2008)

Die Sonografie ist zur Erkennung von Lebermetastasen technisch geeignet. Ihr Routineeinsatz ist aufgrund der Datenlage nicht gesichert. Die Expertenkommission bewertet die Sonografie als einfachstes und kostengünstigstes Verfahren und schlägt daher ihre Nutzung zur Diagnostik auf Lebermetastasen vor.

Empfehlungsgrad A, Level of Evidence 5, [960, 962, 976], starker Konsens

\section{Hintergrund}

Die Sensitivität der Sonografie zum Nachweis von Lebermetastasen schwankt in einem weitem Bereich zwischen 53\% und $82 \%$ [976]. Sie ist der Computertomografie in dieser Hinsicht in den meisten Studien unterlegen. In einer kontrollierten randomisierten Studie [962] erwies sich der Einsatz von Sonografie und Computertomografie ohne Einfluss auf Überleben und Resektionsrate der nachgesorgten Patienten. Lediglich in einer Metaanalyse mehrerer randomisierter Studien stellte sich der Einsatz eines bildgebenden Verfahrens zur Beurteilung der Leber als statistisch signifikanter Überlebensvorteil dar [960]. Erfolgte die Berechnung dieses Ergebnisses nicht als Odds Ratio, sondern als Risikodifferenz, so war dieser Vorteil nicht mehr nachweisbar [960]. Da die Sonografie schneller und kostengünstiger als andere bildgebende Verfahren ist, wurde von den Teilnehmern der Konsensuskonferenz der abdominelle Ultraschall zur Entdeckung von Lebermetastasen in der Nachsorge empfohlen.

\subsubsection{Endosonografie}

10.9. Empfehlung (2008)

Die Endosonografie (EUS) ist zur Erkennung von Lokalrezidiven beim Rektumkarzinom insbesondere in Kombination mit der EUSgesteuerten Biopsie geeignet. Zum routinemäßigen primären Einsatz in der Nachsorge kann derzeit keine Empfehlung gegeben werden.

Empfehlungsgrad B, Level of Evidence 3b, [987], starker Konsens

\section{Hintergrund}

Endosonografische Nachsorgeuntersuchungen beim Rektumkarzinom erwiesen sich in einer Studie zur Erkennung von lokoregionären Rezidiven nach sphinktererhaltender Rektumresektion als hilfreich, wenn dieses Verfahren mit einer EUS-gesteuerten Biopsie kombiniert wurde [987]. 68 perirektalen Läsionen in der EUS standen 36 tatsächliche Lokalrezidive bei 312 Patienten gegenüber. 12 Rezidive konnten mittels Proktoskopie erkannt werden. Bei 22 der endosonografisch gesehenen Läsionen war die Histologie positiv. Bei 41 Läsionen war sie negativ und bei 5 wurde kein verwertbares Ergebnis erhalten. Bei 18 der 68 Patienten beeinflusste die Endosonografie das weitere Vorgehen [987]. Zur primären Diagnostik wird die EUS in der Nachsorge wegen der mit der Biopsie verbundenen Invasivität nicht empfohlen. Sie ist jedoch bei der weiteren Abklärung von mit anderen Methoden entdeckten lokoregionären Rezidiven des Rektumkarzinoms nützlich.

\subsubsection{Röntgen-Thorax-Untersuchung \\ 10.10. Empfehlung (2013)}

Ein Röntgen-Thorax kann bei Patienten mit Rektumkarzinom im Stadium II und III bis zum fünften Jahr jährlich durchgeführt werden. Empfehlungsgrad 0, Level of Evidence 3b, de novo: [988], Konsens

\section{Hintergrund}

In einer Kohortenstudie aus Frankreich traten bei 5,8\% der Patienten innerhalb von 5 Jahren nach kurativer Resektion eines kolorektalen Karzinoms im Verlauf Lungenmetastasen auf [988]. Die Rate an Lungenmetastasen nach 1 Jahr betrug 0,9\%, nach 3 Jahren 4,2\%. Das Risiko von Lungenmetastasen bei Patienten mit Rektumkarzinom war deutlich höher als bei Patienten mit Kolonkarzinom (OR 2,6 95\% CI 1,65-4,75). Das 3-Jahresüberleben bei Nachweis von metachronen Lungenmetastasen betrug 13,8\%. Nach kurativer Resektion dieser Metastasen betrug das relative 3-Jahresüberleben $59 \%$.

In einer systematischen Literatursuche zum Stellenwert der Durchführung des Röntgen-Thorax in der Nachsorge wurden 18 Studien zu diesem Thema identifiziert. Hierbei zeigte sich, dass in der Nachsorge durch einen Röntgen-Thorax bei 0,8 bis $7,0 \%$ aller Patienten Lungenmetastasen bzw. zwischen 3,4 und 29,4\% aller Rezidive durch diese Bildgebung entdeckt wurden. Die Rate an kurativen Resektionen der detektierten Metastasen in diesen Studien betrug 0-100\%. Nähere Aussagen zu Einfluss von Tumorlokalisation und -stadium und idealer Abstand der Röntgenuntersuchungen sind aufgrund der Angaben in den Studien, der geringen Fallzahlen bzw. der Heterogenität der Studien nicht möglich.

Insgesamt scheint der Nutzen der Lungenbildgebung aufgrund der Daten von Mitry bei Patienten mit Rektumkarzinomen höher zu sein, sodass der Einsatz in der Nachsorge von Kolonkarzinompatienten aufgrund des unklaren Nutzens weiterhin nicht empfohlen werden kann.

\subsubsection{Computertomografie}

\subsection{Empfehlung (2013)}

Die Computertomografie ist zur Erkennung von Lebermetastasen, von Lokalrezidiven im kleinen Becken sowie von Lungenmetastasen technisch geeignet. Die Datenlage spricht gegen einen routinemäßigen Einsatz der Computertomografie in der Nachsorge. Empfehlungsgrad B, Level of Evidence 1b, Evidenz aus Aktualisierungsrecherche: [965, 966, 968, 989 - 991], starker Konsens 


\section{Hintergrund}

In einer randomisierten kontrollierten Studie zeigte der Einsatz der Computertomografie (CT) in der Nachsorge keinen Einfluss auf das Überleben der Patienten [966]. Zwar wurden die Leberherde hierdurch etwas früher entdeckt (12 von 20 waren asymptomatisch), aber das CT steigerte nicht die Zahl kurativer Leberresektionen. CEA zeigte in einigen Studien ein Tumorrezidiv früher an als regelmäßige CT-Untersuchungen [965, 966, 991].

Eine Aktualisierung der ASCO Leitlinien im Jahr 2005 führte zur Empfehlung von jährlichen CT-Abdomen-Untersuchungen über 3 Jahre [989]. Die Kommission begründete seine Entscheidung für den Einsatz des Abdomen-CTs in der Nachsorge mit der Arbeit von Chau et al. [990]. Bei 530 Patienten wurden retrospektiv 154 Tumorrezidive ausgewertet. Die Studie war ursprünglich als Therapiestudie und nicht zur Prüfung der Rolle des CTs in der Nachsorge angelegt. 65 Rezidive wurden durch Symptome, 45 durch wiederholte CEA-Bestimmungen und 49 durch ein CT 12 und 24 Monate nach Beginn der adjuvanten Chemotherapie entdeckt. 14 dieser Patienten hatten auch ein erhöhtes CEA und wurden in beiden Gruppen aufgeführt. Häufigere Resektionen erfolgten in der CT-Gruppe $(n=13,26,5 \%)$ und in der CEA-Gruppe $(n=8$, $17,8 \%)$. In der symptomatischen Gruppe wurden 2 Patienten operiert (3,1\%). Diese Unterschiede waren signifikant. Die Wertigkeit der Sonografie im Vergleich zum CT wurde jedoch nicht untersucht. Auch neuere Metaanalysen lassen keinen Rückschluss auf die Art der einzusetzenden Verfahren zu [968], sodass derzeit keine Empfehlung zu regelmäßigen CT-Untersuchungen bei asymptomatischen Patienten in der Nachsorge gemacht werden kann.

\subsubsection{Endoskopische Verfahren}

10.3.9.1. Koloskopie

\subsection{Empfehlung (2013)}

Die Koloskopie ist geeignet, Lokalrezidive oder Zweittumoren zu erkennen. Alle Patienten sollten prä- oder innerhalb von 6 Monaten postoperativ eine komplette Koloskopie erhalten.

Eine Koloskopie sollte nach $1 \mathrm{Jahr}$ und anschließend bei unauffälligem Befund alle 5 Jahre erfolgen, um metachrone Karzinome oder Polypen zu erkennen. Ist die komplette Koloskopie postoperativ im Zeitraum von 6 Monaten erfolgt, sollte die nächste Koloskopie nach 5 Jahren durchgeführt werden.

Findet sich bei der Koloskopie nach 6 Monaten oder 12 Monaten eine Neoplasie, sollte entsprechend Kapitel 6.5 verfahren werden. Empfehlungsgrad B, Level of Evidence 2b, de novo: [961, $992-$ 995], starker Konsens

\subsubsection{Sigmoidoskopie}

\subsection{Empfehlung (2008)}

Die Sigmoidoskopie ist geeignet, Lokalrezidive und Zweittumoren im eingesehenen Bereich zu erkennen. Zusätzliche Sigmoidoskopien sind nur beim Rektumkarzinom vom UICC-Stadium II und III bei Patienten durchzuführen, die keine neoadjuvante oder adjuvante Radiochemotherapie erhielten.

Empfehlungsgrad B, Level of Evidence 4, starker Konsens

\subsubsection{Rektoskopie}

\subsection{Empfehlung (2008)}

Die starre Rektoskopie ist geeignet, beim Rektumkarzinom Lokalrezidive und Anastomosenveränderungen zu erkennen. Sie kann als alternatives Verfahren zur Sigmoidoskopie angewandt werden. Empfehlungsgrad B, Level of Evidence 4, starker Konsens

\section{Hintergrund}

Ziel einer endoskopischen Nachsorge ist es, metachrone Neoplasien und Anastomosenrezidive in einem kurativem Zustand $\mathrm{zu}$ entdecken. Hierbei sollte bedacht werden, dass in einer Metaanalyse verschiedener randomisierter kontrollierter Studien gezeigt wurde, dass die koloskopische Nachsorge weniger effektiv als die Suche nach extramuralen Rezidiven ist [961]. Ferner geht die Koloskopie mit Kosten und einer - wenn auch geringen - Komplikationsrate einher.

In den im Rahmen der Literatursuche identifizierten Artikeln (für Einzelheiten siehe Evidenzbericht) variierte die Rate an metachronen Karzinomen zwischen 0 und 6,4\% und die der Anastomosenrezidive zwischen 0 und $12 \%$ innerhalb des Follow-up (24-94 Monate). In den Studien, in denen die entsprechenden Angaben vorhanden waren, betrug die errechnete jährliche Inzidenz metachroner Karzinome 0-2,3\%. Berücksichtigt man zusätzlich endoluminale Anastomosenrezidive betrug die jährliche Inzidenz von Karzinomen 0 bis 2,6\%. Die Rate fortgeschrittener Adenome, die im Rahmen der Nachsorge detektiert wurde, wurde nur in wenigen Studien angegeben und variierte zwischen 3,7 und $13 \%$ innerhalb des Follow-up.

Die Rate an kurativen Resektionen der metachronen Karzinome und/oder Anastomosenrezidive betrug 27 bis $100 \%$.

Die erforderliche Häufigkeit von Koloskopien in der Nachsorge wurde in einer Studie untersucht, die eine intensive koloskopische Nachsorge (im ersten Jahr alle 3 Monate, im 2. und 3. Jahr alle 6 Monate und danach jährlich) mit einem weniger intensiven Protokoll (nach 6, 30 und 60 Monaten) verglich [992]. Rezidive wurden bei $8,1 \%$ der intensiv nachgesorgten und bei $11,4 \%$ der weniger häufig koloskopierten Patienten gefunden. Während sich das Gesamtüberleben nicht unterschied, zeigte sich eine statistische Signifikanz für mehr asymptomatische Rezidive und eine bessere Prognose der erneut operierten Patienten. Die Häufigkeit von metachronen Tumoren wird in dieser Studie nicht angegeben. Die hohe Frequenz an Koloskopien wirkte sich nicht auf das Gesamtüberleben aus, zumal die meisten Rezidive extraluminal entstehen, sodass ein weniger belastendes Protokoll für die Patienten ausreichend effektiv ist.

In einer Studie wiesen 20 von 1002 Patienten (3,1\%) ein Zweitkarzinom auf, wovon 9 innerhalb von 18 Monaten entdeckt wurden [993]. Eine fortgeschrittene Neoplasie (definiert als Adenom $>1 \mathrm{~cm}$, villöse Histologie, HGD oder Karzinom) fand sich häufiger $(15,5 \%)$ bei einer Koloskopie nach 36 - 60 Monaten im Vergleich zu einer frühzeitigeren Untersuchung innerhalb von 18 Monaten (6,9\%). Eine frühzeitigere Koloskopie war zwar nicht mit einem besseren Überleben in der Nachsorge assoziiert, die Autoren kommen aber zu dem Schluss, dass eine erste Koloskopie 12 Monate nach der Operation gemacht werden sollte, um möglicherweise bei der Indexuntersuchung übersehene metachrone Karzinome rechtzeitig zu entdecken. Aufgrund dieser Daten erfolgt jetzt die Empfehlung die erste Nachsorgekoloskopie 12 Monate nach dem Zeitpunkt der Operation durchzuführen.

Auch Rex et al. betonen, dass das primäre Ziel der Koloskopie in der Nachsorge weniger die Entdeckung eines seltenen Anastomosenrezidivs als das Auffinden metachroner Neoplasien ist [994]. 2 - 7\% der Patienten wiesen mindestens einen metachronen Tumor auf. Inwieweit es sich um echte metachrone Läsionen handelte oder um übersehene synchrone Karzinome bei der ersten Koloskopie, kann anhand der Daten nicht entschieden werden. Dennoch empfehlen auch die Autoren dieser Leitlinie, eine erste Koloskopie 12 Monate nach Tumorresektion durchzuführen. Sie begründen diese Empfehlung mit einer Publikation aus 
dem Jahr 1993 eines Tumorregisters in Nebraska, das eine jährliche Inzidenz von metachronen Tumoren von 0,35\% ergab [937]. Da ein lebenslanges Risiko für metachrone Tumoren besteht, ist eine dauerhafte koloskopische Nachsorge analog der Vorsorge auf ein Kolonkarzinom indiziert [995].

\subsubsection{Kolonkontrasteinlauf, virtuelle Kolonografie und} PET

\subsection{Empfehlung (2013)}

Kolonkontrasteinlauf, virtuelle Kolonografie und PET, PET-CT und PET-MRT haben in der programmierten Nachsorge keinen Stellenwert.

Empfehlungsgrad B, Level of Evidence 4, de novo: [191, 996, 997], starker Konsens

\section{Hintergrund}

Zum Einsatz der PET in der Nachsorge existieren nur wenige Daten. Die überwiegende Mehrzahl der Studien schließt nur Patienten mit V.a. Rezidiv ein und überprüft bei diesen die Wertigkeit der PET. Dieses Vorgehen war nicht Ziel der Literatursuche, sondern es ging um den Einsatz bei asymptomatischen Patienten ohne V.a. Rezidiv.

In einer randomisierten Studie [996] mit sehr engmaschigen Nachsorgeuntersuchungen wurden durch eine PET nach 9 und 15 Monaten Rezidive früher erkannt und konnten häufiger R0reseziert werden.

In einer prospektiv randomisierten Studie [191] sollte die Rolle der PET-CT in der Nachsorge des kolorektalen Karzinoms geklärt werden. Nach 9 und 15 Monaten erfolgte zusätzlich zu einer Nachsorge bestehend aus Abdomensonografie, Röntgen- Thorax, Tumormarkerbestimmung und Abdomen-CT im Vergleich zur Kontrollgruppe eine PET-CT. Die Rezidivrate war in beiden Gruppen nicht unterschiedlich. Rezidive wurden in der PET-Gruppe im Mittel 3,2 Monate früher entdeckt. Zwar wurden häufiger Rezidivoperationen in der PET-CT Gruppe induziert (15 vs. 2), eine Aussage zu einem besseren Überleben konnte aber nicht gemacht werden, da die Studie vorzeitig mit Erscheinen einer neuen PETCT Generation abgebrochen wurde. Bedenklich ist auch, dass in drei Fällen falsch positive Befunde durch die PET-CT erhoben wurden, was zu unnötigen diagnostischen Maßnahmen oder operativen Eingriffen führte.

In einer prospektiven Fallserie von 31 Patienten [997] wurde eine PET nach 2 Jahren durchgeführt. Die Patienten hatten zuvor regelmäßig CT/MRT erhalten und galten als rezidivfrei. Bei 6 Patienten war die PET positiv, bei 5 Patienten konnte ein Rezidiv im Verlauf gesichert werden. Es bleibt unklar, wie das Outcome dieser Pat. war. Insgesamt wurde die Datenlage durch die Teilnehmer als nicht ausreichend eingeschätzt, um die PET/PET-CT/PET-MRT-Untersuchung in die Routinenachsorge aufzunehmen.

Kolonkontrasteinlauf und die virtuelle Kolonografie wurden in der Nachsorge bisher nicht evaluiert und können die endoskopischen Verfahren in der Nachsorge nicht ersetzen.

\subsection{Zeitlicher Ablauf der Nachsorge}

10.16. Konsensbasierte Empfehlung (2013)

Der Beginn der Nachsorge errechnet sich ab dem Zeitpunkt der Operation.

GCP, starker Konsens

\section{Hintergrund}

In den ersten beiden Jahren nach der Operation eines kolorektalen Karzinoms treten $80 \%$ der Rezidive auf, wobei nach 5 Jahren praktisch keine neuen Rezidive mehr festgestellt werden [998]. Dies gilt auch für das Rektumkarzinom, obwohl bei dieser Tumorlokalisation auch später noch lokoregionäre Rezidive beobachtet wurden [943]. Dies rechtfertigt jedoch nicht, die Nachsorge über das 5. postoperative Jahr hinaus auszudehnen.

In den meisten Studien wurden die Nachsorgeintervalle im 1. und 2. postoperativen Jahr mit 3 Monaten kürzer gehalten als in den darauffolgenden Jahren [962, 963, 965, 966]. 3-monatliche Intervalle erwiesen sich in einer Studie bei ansonsten gleichen Untersuchungsmethoden besser als Kontrollen in 6-monatlichen Abständen [965]. Allerdings erhielten die Patienten in der 3-monatlich nachgesorgten Gruppe jährlich noch zusätzlich ein CT, das sich in einer anderen Studie in der Nachsorge jedoch als nutzlos erwies [966]. Die Konsensuskonferenz entschied sich bei Fehlen eindeutiger Daten für Untersuchungsintervalle von 6 Monaten in den ersten 2 Jahren. Über den Zeitpunkt nach 5 Jahren sollten lediglich Koloskopien zum Ausschluss eines Zweitkarzinoms erfolgen.

\subsection{Altersbegrenzung der Nachsorge}

In kontrollierten Studien zur Nachsorge wurden Patienten bis 87 Jahre eingeschlossen [962 - 967]. Aus diesen Studien kann keine Altersbegrenzung abgeleitet werden. Sinnvollerweise sollten jedoch Operabilität, biologisches Alter, Begleiterkrankungen und der Wille, sich gegebenenfalls erneut operieren zu lassen, die Art und Dauer der Nachsorge bestimmen.

\subsection{Sonderfälle}

\subsection{Empfehlung (2008)}

Nach lokaler Abtragung eines pT1-Low-Risk Karzinoms sollten lokale endoskopische Befundkontrollen nach 6, 24 und 60 Monaten erfolgen (s. auch Kapitel 6.4).

Empfehlungsgrad B, Level of Evidence 4, starker Konsens

\subsection{Konsensbasierte Empfehlung (2008)}

Nach palliativer Tumorresektion (R2-Resektion) sind programmierte Nachsorgeuntersuchungen nicht erforderlich.

GCP, starker Konsens

\subsection{Konsensbasierte Empfehlung (2013)}

Patienten im Stadium IV nach kurativer Metastasentherapie sollten einer programmierten Nachsorge unterworfen werden. GCP, starker Konsens

\subsection{Empfehlung (2008)}

Bei Patienten mit einem Karzinom bei HNPCC sind nach Hemikolektomie koloskopische Untersuchungen und nach subtotaler Kolektomie rektoskopische Untersuchungen in jährlichen Intervallen angezeigt (s. auch Empfehlung 5.29).

Empfehlungsgrad A, Level of Evidence 2a, starker Konsens

\subsection{Empfehlung (2008)}

Bei Patienten mit einem Kolonkarzinom bei familiärer Adenomatosis coli (FAP) sollten nach Anlage eines Ileum-Pouches jährlich eine Pouchoskopie durchgeführt werden (s. auch Empfehlung 5.35)

Empfehlungsgrad A, Level of Evidence 2a, starker Konsens 


\subsection{Empfehlung (2008)}

Nach Ileorektostomie ist die Rektoskopie in regelmäßigen Abständen erforderlich (s. auch Empfehlung 5.35)

Empfehlungsgrad A, Level of Evidence 2a, starker Konsens

\subsection{Rehabilitation nach Resektion eines kolorektalen Karzinoms}

10.23. Konsensbasierte Empfehlung (2013)

Nach Abschluss der Primärtherapie sollte eine AHB allen rehabilitationsfähigen Patienten angeboten werden. Wenn die AHB unmittelbar postoperativ erfolgt, muss sichergestellt werden, dass eine indizierte adjuvante Chemotherapie zeitgerecht eingeleitet wird. Alternativ kann die AHB nach Abschluss der adjuvanten Chemotherapie erfolgen.

GCP, starker Konsens

\section{Hintergrund}

Auftrag der Rehabilitation ist die möglichst weitgehende Beseitigung tumor- oder therapiebedingter Folgen sowie die Hilfestellung bei der Akzeptanz verbleibender Behinderungen mit dem Ziel einer selbstbestimmten Teilhabe am beruflichen, privaten und sozialen Leben. Zum Stellenwert rehabilitativer Maßnahmen bei Patienten mit kolorektalem Karzinom liegt keine auswertbare Literatur vor. Für die rehabilitative Maßnahme sind ausgewiesene Reha-Zentren bzw. Kliniken mit gastrointestinaler und onkologischer Expertise zu bevorzugen, die den Standards des Qualitätssicherungsverfahrens der DRV und Anforderungen von Darmzentren entsprechen.

Der Rehabilitationsbedarf nach Behandlung von kolorektalen Tumoren ist äußerst variabel und im Wesentlichen abhängig von Art und Ausmaß des operativen Vorgehens sowie der Therapiefolgen (Kontinenzprobleme, sexuelle Funktionsstörungen, Stoma usw.).

Eine psychosoziale Beratung und gegebenenfalls Betreuung ist wünschenswert bei Problemen der psychischen Verarbeitung des Tumorleidens, bei Therapiefolgen, bei sozialen Anpassungsstörungen sowie bei der beruflichen Wiedereingliederung [972, 999, 1000].

Kontakte mit erfahrenen Gleichbetroffenen können gerade bei der psychischen Verarbeitung oder der Anpassung an eine veränderte Lebenssituation die wesentliche Hilfe für einen Neubetroffenen darstellen. Gleichbetroffene können durch ihr eigenes Beispiel sowie ihre Erfahrungen im Alltagsleben mit Krankheit und Behinderung glaubwürdig vermitteln, dass eine hohe Lebensqualität auch dann möglich ist. Deswegen sollten Kontaktaufnahmen oder Vermittlung zu Selbsthilfeorganisationen erfolgen.

Grundsätzlich sollten die Patienten in Abstimmung mit dem familiären Umfeld erst nach Abschluss der Primärtherapie in die Rehabilitation gehen. Falls die Rehabilitationsmaßnahme vor einer indizierten adjuvanten Chemotherapie erfolgen soll, muss die Durchführung der Chemotherapie während des Rehabilitationsverfahrens gewährleistet sein. Dazu werden neben dem Operationsbericht einschließlich der pathologischen Begutachtung (Tumorformel) auch die Entscheidung des Tumorboards benötigt und sollten daher der Rehabilitationseinrichtung zur Verfügung stehen.
10.8. Tertiärprävention

10.8.1. Versorgungskontinuität und Fortführung der gesundheitsförderlichen Aktivitäten nach Akuttherapie und Rehabilitation

Auch nach den Phasen der Akuttherapie und einer adjuvanten Chemotherapie sollten alle an der Behandlung und Nachversorgung beteiligten Berufsgruppen, wie z. B. niedergelassenen Ärzte, Pflegekräfte oder Physiotherapeuten die Patienten weiter beraten und begleiten.

\subsubsection{Verbesserung der Versorgungskontinuität}

Ein wichtiges Ziel sollte die Verbesserung der Versorgungskoordination sein, um die vorzeitige Beendigung oder den verzögerten Beginn des nachstationären Therapieverlaufs oder eine regionale Unterversorgung mit Versorgungslücken $\mathrm{zu}$ vermeiden. Das Übergangsmanagement von Patienten zwischen primärer Therapie, Rehabilitation und weiterer Unterstützung im Hinblick auf krankheits- oder therapiebezogene Nebenwirkungen und Beschwerden ist nach wie vor defizitär [1001, 1002]. Insbesondere nach der Entlassung aus der Klinik ist die ambulante Weiterbetreuung der Patienten durch niedergelassene Ärzte und andere Versorger (Psychoonkologie, Physiotherapie, Ergotherapie u.a.) wenig koordiniert. Häufig stehen die Patienten einer Situation von untereinander konkurrierenden und unverbundenen Gesundheitsanbietern gegenüber. „Access“ und „Support“ sind besonders eingeschränkt in strukturschwachen ländlichen Regionen mit niedrigem Versorgungsstand [1003, 1004]. In Studien wurde der Vorteil eines Fall-bezogenen Managements (Case-Management) durch begleitende professionelle Steuerung des Behandlungsund Nachversorgungsverlaufs für den Patienten belegt [1005]. Wie aktuelle systematische Übersichten belegen, kann die Versorgungskontinuität insbesondere durch pflegerische Interventionen optimiert werden [1006, 1007]. Durch multiprofessionelle Steuerung des Behandlungs- und Nachsorgeverlaufs kann eine verbesserte Symptomkontrolle, eine erhöhte Leitlinientreue, eine verbesserte Patientenzufriedenheit und höhere gesundheitsbezogene Lebensqualität erzielt werden [1005 - 1008].

Auch bis zum Beginn der adjuvanten Therapie sollten Patienten durch ein multiprofessionelles „Case Management“ unterstützt werden, um Versorgungskontinuität zu gewährleisten. Als signifikante Risikofaktoren für Versorgungsbrüche im Übergang von primär chirurgischer zu adjuvanter (Radio-)Chemotherapie wurde ein hohes Alter, das Vorliegen von Komorbiditäten, ein niedriger sozioökonomischer Status, die Versorgungsstufe der erstversorgenden Einrichtung und der Zugang oder die Wohnortentfernung zu subspezialisierten Fachärzten identifiziert [1001].

\subsubsection{Fortführung der gesundheitsförderlichen Aktivitäten}

Die Patienten sollten verstärkt dazu angehalten werden, ihre für Gesundheit und Lebensqualität förderlichen Lifestyle-Aktivitäten (s. Kapitel 10.8.1.2) weiter zu praktizieren. Darüber hinaus belegen Studien, dass Patienten davon profitieren, wenn sie das Management ihrer Beschwerden und Nebenwirkungen (Übelkeit, Erbrechen, Schmerz, Fatigue, Depression, Stoma-Versorgung u. a.) selbst in die Hand nehmen können [1009, 1010]. Dies kann durch einfache Maßnahmen wie telefonisches „Follow up“, schriftliches Informationsmaterial, das Führen eines Patiententagebuchs mit integrierter Ergebniskontrolle („Patient Reported Outcomes“, PRO) unterstützt werden [1005, 1007, 1008, 1011]. Bei der Umsetzung dieser Maßnahmen kommt den Krebs-Beratungsstellen sowie den Selbsthilfegruppen eine wichtige Rolle zu. 
10.8.2. Tertiärprävention: Physikalische Aktivität, Ernährung sowie komplementäre und alternative Therapie

10.8.2.1. Physische Aktivität

10.24. Empfehlung (2013)

Kohortenstudien weisen auf einem Zusammenhang von körperlicher Aktivität und erniedrigter Rezidivrate und verbessertem Überleben hin. Patienten sollten zu körperlicher Aktivität angehalten werden.

Empfehlungsgrad B, Level of Evidence 2b, Evidenz aus Aktualisierungsrecherche: [1012 - 1014], Konsens

\section{Hintergrund}

Drei Kohortenstudien [1012 - 1014] zeigen, dass durch körperliche Aktivität nach kurativer Therapie eines kolorektalen Karzinoms sowohl die Rezidivrate als auch die Mortalität abgesenkt werden. Die Aktivität bei unbehandeltem Karzinom beeinflusst diese Parameter nicht. Der positive Effekt körperlicher Aktivität findet sich auch bei übergewichtigen Patienten. Eine alleinige Gewichtsveränderung im Anschluss an die adjuvante Therapie hat dagegen keinen Einfluss auf die Prognose.

Eine statistisch signifikante Risikoreduktion für die krankheitsspezifische Mortalität tritt ab einer wöchentlichen körperlichen Belastung von 3 Met Stunden (metabolic equivalent task) auf.

\subsubsection{Ernährung}

Für die Tertiärprävention gibt es keine Studien, aus denen sich konkrete Ernährungsempfehlungen für Patienten nach kurativer Behandlung eines kolorektalen Karzinoms ableiten lassen.

Für einen gesunden Lebensstil gelten die Empfehlungen der Deutschen Gesellschaft für Ernährung. Ein erhöhter BMI ist nicht mit der Rezidivrate oder dem Überleben korreliert [1012]. Eine Gewichtsabnahme bei übergewichtigen Patienten vermindert die Rezidivrate nicht [1012]. Allerdings sollte bei Vorliegen eines erheblichen Übergewichts im Sinne der allgemeinen Prävention eine Gewichtsreduktion angestrebt werden.

Es gibt keinen Hinweis dafür, dass die Einnahme von Nahrungsergänzungsmitteln (Vitaminen und Spurenelementen) einen positiven Einfluss auf die Rezidivrate hat [1015]. Vitamine und Spurenelemente sollten nur bei nachgewiesenem Mangel substituiert werden.

\subsubsection{Komplementäre und alternative Medizin}

Komplementäre Verfahren beruhen auf unterschiedlichen Methoden und Substanzen, die zum Teil aus der Naturheilkunde stammen oder auf andere Weise Gedanken eines ganzheitlichen Therapiekonzepts verfolgen.

Sie ersetzen keine aktive antitumorale oder supportive Therapie, sondern stellen ergänzende Methoden dar, die es dem Patienten ermöglichen, selbständig tätig zu werden.

Komplementäre Therapien können Neben- und Wechselwirkungen haben. Deshalb ist es sinnvoll, dass eine Beratung zu komplementärmedizinischen Behandlungen durch onkologisch erfahrene Ärzte durchgeführt wird.

Eine der am häufigsten genutzten komplementären Therapien ist die Misteltherapie. Ein Review und das Cochrane-Review [75, 76] fassen zusammen, dass die meisten der bisher veröffentlichten Studien von unzureichender Qualität sind. Die wenigen methodisch gut durchgeführten Studien zeigen keine positiven Effekte auf das Überleben bei verschiedenen Tumorarten. Für das kolorektale Karzinom gibt es zwei retrospektive Untersuchungen, die einen Überlebensvorteil zeigen [1016, 1017]. Beide weisen jedoch deutliche methodische Mängel auf. Sowohl das Cochrane-Review, wie das systematische Review, finden nur schwache Hinweise auf eine Verbesserung der Lebensqualität durch Misteltherapie.

Zum Einfluss der Misteltherapie auf die Lebensqualität ist zuletzt 2010 ein Review aus 26 randomisiert kontrollierten Studien und 10 nicht randomisierten kontrollierten Studien publiziert worden [1018]. Es wurden Verbesserungen v. a. im Hinblick auf Coping, Fatigue, Schlaf, Erschöpfung, Übelkeit, Appetit, Depression und Angst gezeigt. Die von den Autoren als methodisch gut in dem Review charakterisierten Studien weisen allerdings ebenfalls erhebliche Mängel auf.

Für eine Reihe von hauptsächlich pflanzlichen Stoffen liegen präklinisch-experimentelle Daten vor, die auf eine antitumorale Wirkung hindeuten $[1019,1020]$. Diese rechtfertigen einen klinischen Einsatz außerhalb von Studien derzeit nicht. Bisher wurden 2 kleine Studien zu Grünteeextrakt nach Kolonpolyp bzw. Kolonkarzinom [1020] publiziert. In beiden Studien war in der Therapiegruppe die Rate an Adenom- bzw. Karzinomrezidiven signifikant vermindert.

Es gibt eine Reihe von Publikationen zum Einsatz von Heilpilzen bei kurativ behandelten Patienten mit kolorektalem Karzinom [1021, 1022]. Diese Studien stammen aus China und Japan und sind in den Originalsprachen publiziert. Ob die dort durchgehend gezeigten positiven Effekte auf die Überlebensraten wissenschaftlich fundiert sind, ist unklar. Aufgrund seltener aber bedrohlicher Nebenwirkungen ist der Einsatz von Präparaten aus Heilpilzen nicht empfehlenswert.

In pflanzlichen Präparaten aus Asien im Kontext der Traditionellen Chinesischen Medizin und des Ayurveda wurden wiederholt Beimengungen aus Schwermetallen, Pestiziden und medizinischen Substanzen wie Kortikoide und Coumarine gefunden. Der Einsatz ist bei fehlendem Nutzennachweis nicht empfehlenswert.

Ein therapeutischer Nutzen der Homöopathie als supportive Therapie ist beim kolorektalen Karzinom nicht erwiesen. [1007, 1023]. Eine Cochrane-Analyse [1023] schloss eine sehr heterogene Gruppe von Studien ein. Die beiden positiv bewerteten Studien beinhalteten keine Homöopathie im eigentlichen Sinn, sodass insgesamt keine Studie den positiven Effekt der Homöopathie bei Tumorpatienten belegen konnte [1023].

\subsubsection{Alternative Heilmethoden}

Es gibt eine Reihe von „alternativ“ zur wissenschaftlich empfohlenen Therapie angebotenen Therapien, für deren Einsatz es keine Rationale gibt. Hierzu gehören u. a.: Ukrain, Vitamin $B_{17}$ (Aprikosenkerne, Bittermandel), „insulinpotenzierte Therapie“, kohlenhydratarme Diät, ,Vitamine nach Dr. Rath“, „Neue Germanische Medizin ${ }^{\circledR}$, Eigenblutzytokine, Zapper, „Redifferenzierungstherapie“. Es ist wichtig, Patienten vor diesen Angeboten durch klare Stellungnahmen zu schützen ( $\odot$ Tab. 24).

\section{Qualitätsindikatoren}

$\nabla$

Qualitätsindikatoren sind Messgrößen, deren Erhebung der Beurteilung der Qualität der zugrundeliegenden Strukturen, Prozessen bzw. Ergebnisse dient ${ }^{7}$. Das vorrangige Ziel ihres Einsatzes ist die stetige Verbesserung der Versorgung. Qualitätsindikatoren als qualitätsbezogene Kennzahlen sind damit ein wesentlicher

\footnotetext{
7 Ärztliches Zentrum für Qualität in der Medizin (ÄZQ), Gramsch E, Hoppe JD, Jonitz G, Köhler A, Ollenschläger G, Thomeczek C, (eds.). Kompendium QM-A. Qualitätsmanagement in der ambulanten Versorgung. 3 rd ed. Köln: Dt. Ärzte-Verl.; 2008.
} 
Bestandteil des Qualitätsmanagements. Dabei ist zu beachten, dass einzelne Indikatoren jeweils nur einen Aspekt des komplexen Versorgungsgeschehens beleuchten. Der Auswahl geeigneter Indikatoren kommt deshalb große Bedeutung zu.

Als Grundlage für die Qualitätsindikatoren dienten alle starken Empfehlungen (Empfehlungsgrad A) sowie Statements mit hoher Evidenz (LoE 1). Gemäß den methodischen Vorgaben wurden von den Empfehlungen dieser Leitlinie Qualitätsindikatoren ab- geleitet. Die einzelnen methodischen Schritte hierzu finden sich im Leitlinienreport dieser Leitlinie.

Es wurden insgesamt 10 Indikatoren angenommen ( $\bullet$ Tab. 25). Diese sind als vorläufig bewertete Vorschläge zu verstehen. Eine abschließende Bewertung kann erst nach einem erforderlichen Pilottest mit Datenerhebung erfolgen.

Tab. 24 Programmierte Untersuchungen im Rahmen der Nachsorge bei kolorektalem Karzinom UICC II oder III.

\begin{tabular}{|c|c|c|c|c|c|c|c|c|c|c|c|}
\hline \multirow[t]{2}{*}{ Untersuchung } & \multicolumn{11}{|c|}{ Monate } \\
\hline & 3 & 6 & 9 & 12 & 15 & 18 & 21 & 24 & 36 & 48 & 60 \\
\hline Anamnese, körp. Untersuch., CEA & & $x$ & & $x$ & & $x$ & & $x$ & $x$ & $x$ & $x$ \\
\hline Koloskopie & & $x^{1}$ & & $x^{2}$ & & & & & & & $x^{2}$ \\
\hline Abdomensonografie ${ }^{3}$ & & $x$ & & $x$ & & $x$ & & $x$ & $x$ & $x$ & $x$ \\
\hline Sigmoidoskopie (Rektoskopie) ${ }^{4}$ & & $x$ & & $\times$ & & $x$ & & $x$ & & & \\
\hline Spiralcomputertomografie ${ }^{5}$ & $\times$ & & & & & & & & & & \\
\hline Röntgen Thorax ${ }^{6}$ & & & & $\times$ & & & & $x$ & $\times$ & $\times$ & $\times$ \\
\hline
\end{tabular}

${ }^{1}$ Wenn keine vollständige Koloskopie präoperativ erfolgt ist.

${ }^{2}$ Bei unauffälligem Befund (kein Adenom, kein Karzinom) nächste Koloskopie nach 5 Jahren.

${ }^{3}$ Eine Metaanalyse ergab einen Vorteil für ein bildgebendes Verfahren zum Nachweis von Lebermetastasen in der Nachsorge. Aus diesem Grund entschied sich die Expertenkommission, das einfachste und kostengünstigste Verfahren anzuwenden.

${ }^{4}$ Nur beim Rektumkarzinom ohne neoadjuvante oder adjuvante Radiochemotherapie.

${ }^{5}$ Nur beim Rektumkarzinom 3 Monate nach Abschluss der tumorspezifischen Therapie (Operation bzw. adjuvante Strahlen-/Chemotherapie) als Ausgangsbefund.

${ }^{6}$ Nur beim Rektumkarzinom kann jährlich eine Röntgen-Thorax-Uuntersuchung durchgeführt werden.

Tab. 25 Vorschläge für Qualitätsindikatoren.

\begin{tabular}{|c|c|c|}
\hline Qualitätsindikator & Referenz Empfehlung & $\begin{array}{l}\text { Evidenzgrundlage/ } \\
\text { weitere Informationen }\end{array}$ \\
\hline \multicolumn{3}{|l|}{ QI 1: Angabe Abstand mesorektale Faszie } \\
\hline $\begin{array}{l}\text { Z: Alle Patienten mit Angabe des } \\
\text { Abstands zur mesorektalen Faszie } \\
\text { im Befundbericht } \\
\text { N: Alle Patienten mit Rektumkarzinom } \\
\text { und MRT oder Dünnschicht-CT des } \\
\text { Beckens }\end{array}$ & $\begin{array}{l}\text { Empfehlung } 7.17 \text {. } \\
\text { Die Befundbeschreibung soll eine Aussage über den Abstand zur mesorektalen } \\
\text { Faszie beinhalten. }\end{array}$ & $\begin{array}{l}\text { GCP, starker Konsens } \\
\text { Literatur: }[559,560]\end{array}$ \\
\hline \multicolumn{3}{|l|}{ QI 2: Untersuchung Lymphknoten } \\
\hline $\begin{array}{l}\text { Z: Anzahl der Patienten mit } \geq 12 \text { patho- } \\
\text { logisch untersuchten Lymphknoten } \\
\text { N: Alle Patienten mit KRK, die eine } \\
\text { Lymphadenektomie erhalten }\end{array}$ & $\begin{array}{l}\text { Empfehlung } 7.61 . \\
12 \text { und mehr Lymphknoten sollen entfernt und untersucht werden. }\end{array}$ & $\begin{array}{l}\text { GCP, starker Konsens } \\
\text { Literatur: }[579,690,691]\end{array}$ \\
\hline QI 3: Qualität TME & & \\
\hline $\begin{array}{l}\text { Z: Anzahl aller Patienten mit guter oder } \\
\text { moderater Qualität (Grad 1: Mesorektale } \\
\text { Faszie erhalten oder Grad 2: Intrameso- } \\
\text { rektale Einrisse) der TME } \\
\mathrm{N} \text { : Alle Patienten mit radikal operiertem } \\
\text { Rektumkarzinom }\end{array}$ & $\begin{array}{l}\text { Empfehlung 7.66. } \\
\text { Da die Qualität eines Operationsresektats unter Berücksichtigung der oben ge- } \\
\text { nannten Kategorien Rückschlüsse auf die Prognose bezüglich der Entwicklung eines } \\
\text { Lokalrezidives zulässt, soll diese obligat im pathohistologischen Befundbericht wie } \\
\text { folgt beschrieben werden: } \\
\text { Die Qualität des Präparats wird beurteilt an der Integrität der mesorektalen Faszie } \\
\text { im Falle der Resektion mit den } 3 \text { Kategorien: } \\
\text { Mesorektale Faszie erhalten } \\
\text { Intramesorektale Einrisse } \\
\text { Erreichen der Muscularis propria oder Tumor. } \\
\text { Im Falle einer Rektumexstirpation sind bei einer vollständigen Resektion der Leva- } \\
\text { tormuskulatur Präparateinrisse und ein tumorpositiver zirkumferenzieller Sicher- } \\
\text { heitsabstand seltener. } \\
\text { Im pathohistologischen Befundbericht ist deshalb die Beschreibung bezüglich der } \\
\text { Radikalität im Bereich der Levatormuskulatur obligat. Hierzu sollen folgende Kate- } \\
\text { gorien Verwendung finden: } \\
\text { Teile der Muscularis propria fehlen oder Eröffnung des Darmes oder des Tumors } \\
\text { Muscularis propria erhalten, keine Eröffnung des Darmes oder des Tumors } \\
\text { Levatormuskulatur mitreseziert, keine Eröffnung des Darmes oder des Tumors } \\
\text { Diese Bewertungen sind vom Pathologen vorzunehmen. }\end{array}$ & $\begin{array}{l}\text { GCP, starker Konsens } \\
\text { Literatur: [584, 702, 705, } \\
706]\end{array}$ \\
\hline
\end{tabular}


Tab. 25 (Fortsetzung)

\section{Qualitätsindikator}

Referenz Empfehlung

Evidenzgrundlage/

weitere Informationen

\section{QI 4: Angabe Resektionsrand}

Z: Anzahl der Patienten, bei denen der Abstand des aboralen Tumorrands zur aboralen Resektionsgrenze in $\mathrm{mm}$ und der Abstand des Tumors zur zirkumferenziellen mesorektalen Resektionsebene in mm dokumentiert wurden. $\mathrm{N}$ : Alle Patienten mit RK, bei denen der Primärtumor in Form einer TME oder PME reseziert wurde.

\section{Q1 5: Vorstellung Tumorkonferenz}

Z: Anzahl der Patienten, die prätherapeutisch in einer interdisziplinären Tumorkonferenz vorgestellt wurden $\mathrm{N}$ : Alle Patienten mit Rektumkarzinom und alle Pat. mit Kolonkarzinom Stad. IV

\section{Empfehlung 7.65.}

Der zirkumferenzielle Sicherheitsabstand ist negativ, wenn er $1 \mathrm{~mm}$ oder mehr beträgt (R0 „wide“). Ein positiver zirkumferenzieller Sicherheitsabstand liegt vor, wenn der zirkumferenzielle Sicherheitsabstand weniger als $1 \mathrm{~mm}$ beträgt ( $\mathrm{R} 0$ " close") oder Tumorgewebe direkt an ihn heranreicht. Der gemessene Abstand soll in Zahlen dokumentiert werden.
GCP, starker Konsens

Literatur: [560, $702-704]$
Empfehlung 7.1.

Alle Patienten mit KRK sollen nach Abschluss der Primärtherapie (z. B. Operation, Chemotherapie) in einer interdisziplinären Tumorkonferenz vorgestellt werden.

Bereits prätherapeutisch sollen Patienten in folgenden Konstellationen vorgestellt werden:

\section{GCP, Konsens}

Literatur: [517 - 519]

- mit Rektumkarzinom

- mit Kolonkarzinom. im Stadium IV

- mit metachronen Fernmetastasen

- mit Lokalrezidiven

- vor jeder lokal ablativen Maßnahme RFA/LITT/SIRT

\section{QI 6: Adjuvante Chemotherapie}

\section{Z: Anzahl der Patienten, die eine adju-} vante Chemotherapie erhalten haben. $\mathrm{N}$ : Alle Patienten mit Kolonkarzinom im UICC-Stadium III, bei denen eine R0-Resektion des Primärtumors erfolgte

\section{QI 7: Neoadjuvante Radio- und Radiochemotherapie}

Z: Anzahl der Patienten, die eine neoad- Empfehlung: 8.10. juvante Radio- oder Radiochemotherapie erhalten haben

$\mathrm{N}$ : Alle Patienten mit Rektumkarzinom des mittleren und unteren Drittels und den TNM-Kategorien cT3, 4/cM0 und/ oder CN1, 2/cM0, die operiert wurden (= klinisches UICC-Stadium II u. III).

\section{QI 8: Anastomoseninsuffizienz Rektumkarzinom}

\section{Z: Anzahl der Patienten mit Anastomo- Anmerkung:}

seninsuffizienz Grad B (mit Antibiotikagabe o. interventioneller Drainage o. transanaler Lavage/Drainage) oder C

((Re-)-Laparotomie) nach elektiven Eingriffen

$\mathrm{N}$ : Alle Patienten mit Rektumkarzinom, bei denen in einer elektiven primären

Tumorresektion eine Anastomose angelegt wurde.

\section{QI 9: Anastomoseninsuffizienz Kolonkarzinom}

\section{Z: Re-Interventionsbedürftige Anasto-} moseninsuffizienzen Kolon nach elektiven Eingriffen

$\mathrm{N}$ : Alle Patienten mit Kolonkarzinom, bei sich die Aufnahme dieses QI auch ohne konsentierte starke Empfehlung in der Leitdenen in einer elektiven Tumorresektion linie.

eine Anastomose angelegt wurde.

Q/ 10: Anzeichnung Stomaposition

Z: Anzahl der Patienten mit präoperativer Anzeichnung der Stomaposition $\mathrm{N}$ : Alle Patienten mit Rektumkarzinom, bei denen eine Operation mit Stomaanlage durchgeführt wurde

\section{Empfehlung: 7.42}

Die Stomaposition soll präoperativ angezeichnet werden.
Empfehlung LoE 1a, starker Konsens

Literatur: $[738,742-746]$
Empfehlung LoE 1b, starker Konsens

Literatur: [801-803] Die Leitlinienkommission hat beschlossen, dass nicht nur Strukturqualitätsziele, sondern auch Ergebnisqualitätsziele berücksichtigt werden sollen. Daraus ergibt sich die Aufnahme dieses QI auch ohne konsentierte starke Empfehlung in der Leitlinie.
Im UICC-Stadium II und III ist die neoadjuvante Radio- oder Radiochemotherapie indiziert. Eine Sondersituation besteht bei cT1/2-Karzinomen mit fraglichem ymphknotenbefall; hier ist auch die primäre Operation (mit ggf. adjuvante Radiochemotherapie bei $\mathrm{pN}+$ ) eine mögliche Behandlungsoption.

(LoE 1b, EG A)
Anmerkung:

Die Leitlinienkommission hat beschlossen, dass nicht nur Strukturqualitätsziele,

sondern auch Ergebnisqualitätsziele berücksichtigt werden sollen. Daraus ergibt

Bei Patienten mit einem R0-resezierten Kolonkarzinom im Stadium III ist eine

it-


Tab. 26 UICC-Klassifikation des kolorektalen Karzinoms.

\begin{tabular}{llll} 
UICC 2010 & T & $\mathbf{n}$ & M \\
Stadium 0 & Tis & N0 & M0 \\
\hline Stadium I & T1/T2 & N0 & M0 \\
\hline Stadium II & T3/T4 & N0 & M0 \\
IIA & T3 & N0 & M0 \\
IIB & T4a & N0 & M0 \\
IIC & T4b & N0 & M0 \\
Stadium III & jedes T & N1 / N2 & M0 \\
IIIA & T1/T2 & N1 & M0 \\
& T1 & N2a & M0 \\
IIIB & T3/T4 & N1 & M0 \\
& T2/T3 & N2a & M0 \\
IIIC & T1/T2 & N2b & M0 \\
& T4a & N2a & M0 \\
& T3/T4a & N2b & M0 \\
Stadium IV & T4b & N1 $/$ N2 & M0 \\
IVA & jedes T & jedes N & M1 \\
IVB & jedes T & jedes N & M1a \\
& jedes T & jedes N & M1b
\end{tabular}

\section{Anhang}

12.1. UICC-Klassifikation des kolorektalen Karzinoms

Die UICC-Stadieneinteilung wurde von der „Union Internationale Contre le Cancer" (UICC) eingeführt. Sie basiert auf statistischen Untersuchungen und gibt Auskunft über die Ausbreitung der Tumorerkrankung. Sie ist Grundlage bei Prognose- und Therapieplanerstellung ( $\odot$ Tab. 26).

\subsection{Amsterdam-Kriterien (o Tab. 27)}

Tab. 27 Amsterdam-Kriterien (AC).

Amsterdam-Kriterien (AC)
$\begin{array}{ll}\mathbf{A C 1}= & \text { nur KRK, } \mathbf{A C 2}=\text { auch extrakolische Manifestationen [234, 1025] } \\ \text { 1. } & \begin{array}{l}\text { Mindestens drei Familienmitglieder mit HNPCC-assoz. Karzinomen } \\ \text { (Kolon/Rektum, Endometrium, Dünndarm, Urothel (Ureter/Nie- } \\ \text { renbecken) }\end{array} \\ \text { 2. Mindestens zwei aufeinander folgende Generationen betroffen } \\ \text { 3. Ein Familienmitglied erstgradig verwandt mit den beiden anderen } \\ \text { 4. Ein Erkrankter zum Zeitpunkt der Diagnose jünger als } 50 \text { Jahre } \\ \text { 5. Ausschluss einer familiären adenomatösen Polyposis }\end{array}$

\subsection{Revidierte Bethesda-Kriterien}

Tumoren von Patienten, die eines der folgenden Kriterien erfüllen, sollten auf eine Mikrosatelliteninstabilität untersucht werden (๑ Tab. 28):
Tab. 28 Revidierte Bethesda-Kriterien.

\section{Revidierte Bethesda-Kriterien [246]}

Patient mit KRK vor dem 50. Lj.

Patient mit syn- oder metachronen kolorektalen oder anderen HNPCCassoziierten Tumoren (Kolon, Rektum, Endometrium, Magen, Ovar, Pankreas, Ureter, Nierenbecken, biliäres System, Gehirn (v. a. Glioblastom), Haut (Talgdrüsenadenome und -karzinome, Keratoakanthome, Dünndarm) unabhängig vom Alter bei Diagnose.

Patient mit KRK vor dem 60. Lj. mit typischer Histologie eines MSI-H- Tumors (Tumor-infiltrierende Lymphozyten, Crohn's like Lesions, muzinöse oder siegelringzellige Differenzierung, medulläres Karzinom).

Patient mit KRK, der einen Verwandten 1. Grades mit einem KRK oder einem HNPCC-assoziierten Tumor vor dem 50. Lebensjahr hat.

Patient mit KRK (unabhängig vom Alter), der mindestens zwei Verwandte 1. oder 2. Grades hat, bei denen ein KRK oder ein HNPCC-assoziierter Tumor (unabhängig vom Alter) diagnostiziert wurde.

\section{Abbildungsverzeichnis(o Tab. 29)}

\section{Tab. 29}

- Abb. 1 Übersicht zur Graduierung von Empfehlungen 760

- Abb. 2 Algorithmus zum Ablauf der genetischen Diagnostik bei 781 Patienten mit V. a. ein hereditäres Tumordispositions-Syndrom am Beispiel des HNPCC/Lynch-Syndroms. Zum Nachweis der MSI bei $V$. a. HNPCC/Lynch-Syndroms wird auf $\bullet$ Abb. 3 verwiesen. - Abb. 3 Algorithmus zum Ablauf der molekularpathologischen Abklärung eines Mismatch-Reparaturdefekts bei klinischem V. a. HNPCC/Lynch-Syndrom. Zur sich ggf. anschließenden genetischen Diagnostik wird auf $\bigcirc$ Abb. 2 verwiesen.

- Abb. 4 Beurteilung des zirkumferentiellen Resektionsrands.

\section{Tabellenverzeichnis (o Tab. 30)}

\section{$\nabla$}

\section{Tab. 30}

- Tab. 1 Zusammensetzung der Arbeitsgruppen für die Aktualisierung der Kapitel 3, 4, 5, 7, 10

- Tab. 2 Zusammensetzung der AG Qualitätsindikatoren

- Tab. 3 Schema der Evidenzgraduierung nach Oxford

(Version 2009)

\section{- Tab. 4 Schema der Empfehlungsgraduierung}

- Tab. 5 Klassifikation der Konsensusstärke

- Tab.6 Empfohlenes Krebsfrüherkennungsprogramm bei HNPCC 782

- Tab. 7 Klassifikation der Ausprägung der Duodenalpolyposis nach 782 der Spigelman-Klassifikation (modifiziert nach [307])

- Tab. 8 Staging beim kolorektalen Karzinom

- Tab.95-Jahresgesamtüberleben nach R0-Resektion pulmonaler 810 Metastasen (exemplarisch nach Pfannschmidt et al. [833]).

- Tab. 10 Überleben nach Resektion kolorektaler Metastasen 810

- Tab. 11 Überleben nach Resektion kolorektaler Lebermetastasen 810 (nach Fong et al. Annals of Surgery, 1999) [839]

- Tab. 12 Prospektive Studien zur neoadjuvanten Therapie bei 811 Patienten mit resektablen Lebermetastasen

- Tab. 13 Prospektive Studien zur adjuvanten Therapie nach 812

R0-Resektion kolorektaler Lebermetastasen

- Tab. 14 Ansprechraten und Überleben von Patienten nach

Erreichen einer sekundären Resektabilität (ergänzt nach [862])

- Tab. 15 Capecitabin (Cape) im Vergleich mit Bolus 5-FU/FS in der 817

Erstlinientherapie 


\begin{tabular}{|c|c|}
\hline $\begin{array}{l}\text { Tab. } 16 \text { Oxaliplatinhaltige Protokolle in der Erstlinientherapie - } \\
\text { Phase-III-Studien }\end{array}$ & 817 \\
\hline $\begin{array}{l}\text { Tab. } 17 \text { Irinotecanhaltige Protokolle in der Erstlinientherapie - } \\
\text { Phase-III-Studien }\end{array}$ & 818 \\
\hline $\begin{array}{l}\text { Tab. } 18 \text { Oxaliplatin-versus irinotecanhaltige Protokolle in der } \\
\text { Erstlinientherapie }\end{array}$ & 818 \\
\hline $\begin{array}{l}\text { Tab. } 19 \text { Capecitabin und Oxaliplatin-Kombinationen in der } \\
\text { Erstlinientherapie - Phase-III-Studien }\end{array}$ & 819 \\
\hline $\begin{array}{l}\text { Tab. } 20 \text { Capecitabin in Kombination mit Irinotecan in der } \\
\text { Erstlinientherapie - Phase-III-Studien }\end{array}$ & 819 \\
\hline - Tab. 21 FOLFOXIRI in der Erstlinientherapie & 820 \\
\hline - Tab. 22 Studien zur Frage der Therapiepause/Erhaltungstherapie & 822 \\
\hline - Tab. 23 Oxaliplatinhaltige Protokolle in der Zweitlinientherapie & 822 \\
\hline $\begin{array}{l}\text { Tab. } 24 \text { Programmierte Untersuchungen im Rahmen der } \\
\text { Nachsorge bei kolorektalem Karzinom UICC II oder III }\end{array}$ & 831 \\
\hline - Tab. 25 Vorschläge für Qualitätsindikatoren & 831 \\
\hline - Tab. 26 UICC-Klassifikation des kolorektalen Karzinoms & 833 \\
\hline Tab. 27 Amsterdam-Kriterien & 833 \\
\hline - Tab. 28 Revidierte Bethesda-Kriterien & 833 \\
\hline
\end{tabular}

\section{Institute}

Medizinische Klinik, Knappschaftskrankenhaus GmbH Bochum, RuhrUniversität Bochum, Bochum

2 Institut für Humangenetik, Universitätsklinik Bonn, Bonn

3 Institut f. Ernährungsmedizin/Prävention Universität Hohenheim, Stuttgart

${ }^{4}$ Medizinische Klinik I, Krankenhaus St. Franziskus, Mönchengladbach

5 Deutsche ILCO e.V., Bonn

${ }^{6}$ Medizinische Klinik und Poliklinik III, Klinikum der Universität München, München

Chirurgische Klinik, Universitätsklinikum Erlangen, Erlangen

8 Medizinische Klinik I, Klinikum Landshut gGmbH, Landshut

${ }^{9}$ Komplementäre Onkologie, Centrum für Tumorerkrankungen J. W. GoetheUniversität, Frankfurt

10 Medizinische Klinik und Poliklinik II, Klinikum der Universität München, München

11 Chirurgische Klinik I, Charité- Universität Berlin, Campus Benjamin Fränklin, Berlin

12 Medizinische Klinik II, Klinikum Bremen-Mitte, Bremen

13 Medizinische Klinik, Klinikum Bremen-Ost, Bremen

14 Chirurgische Klinik, Universitätsklinikum Mannheim GmbH, Mannheim

15 Institut f. Humangenetik u. Anthropologie, Heinrich Heine Universität, Düsseldorf

16 Abteilung für Hämatologie/Onkologie, St. Josef Hospital Bochum, RuhrUniversität Bochum, Bochum

7 Stiftung Lebensblicke, Ludwigshafen

18 Strahlenklinik, Universität Erlangen/Nürnberg, Erlangen

${ }^{19}$ Gastroenterologische Schwerpunktpraxis, Heidelberg

20 Gastroenterologische Klinik, Stiftung Juliusspital Würzburg, Würzburg

21 I. Med. Abteilung, Städtisches Krankenhaus München-Neuperlach, München

22 Klinik und Poliklinik für Innere Medizin IV, Universität Halle, Halle

23 Klinik für Innere Medizin, Klinikum Arnsberg, St. Johannes-Hospital, MVZ Arnsberg

24 Pathologisches Institut der Ruhr Universität Bochum, Bochum

\section{Literatur}

1 Kirkegaard $\mathrm{H}$ et al. Association of adherence to lifestyle recommendations and risk of colorectal cancer: a prospective Danish cohort study. BMJ 2010; 341: c5504

2 Halle $M$, Schoenberg $M H$. Physical activity in the prevention and treatment of colorectal carcinoma. Dtsch Arztebl Int 2009; 106 (44): 722 727

3 Wolin KY et al. Physical activity and colon cancer prevention: a metaanalysis. Br J Cancer 2009; 100 (4): 611 - 616

4 Wolin $K Y$, Yan $Y$, Colditz GA. Physical activity and risk of colon adenoma: a meta-analysis. Br J Cancer 2011; 104 (5): 882 - 885

5 Wolin $\mathrm{KY}$ et al. Leisure-time physical activity patterns and risk of colon cancer in women. Int J Cancer 2007; 121 (12): 2776-2781
6 Friedenreich $C$ et al. Physical activity and risk of colon and rectal cancers: the European prospective investigation into cancer and nutrition. Cancer Epidemiol Biomarkers Prev 2006; 15 (12): 2398 - 2407

7 Lubin F et al. Nutritional and lifestyle habits and water-fiber interaction in colorectal adenoma etiology. Cancer Epidemiol Biomarkers Prev 1997; 6 (2): $79-85$

8 Giacosa A et al. Energy intake, overweight, physical exercise and colorectal cancer risk. Eur J Cancer Prev 1999; 8 (Suppl 1): S53 - S60

9 Friedenreich CM, Orenstein MR. Physical activity and cancer prevention: etiologic evidence and biological mechanisms. J Nutr 2002; 132 (Suppl 11): 3456S - 3464S

10 Terry $M B$ et al. Risk factors for advanced colorectal adenomas: a pooled analysis. Cancer Epidemiol Biomarkers Prev 2002; 11 (7): 622 - 629

11 Lee IM. Physical activity and cancer prevention - data from epidemiologic studies. Med Sci Sports Exerc 2003; 35 (11): 1823-1827

12 Wei EK et al. Comparison of risk factors for colon and rectal cancer. Int J Cancer 2004; 108 (3): 433 - 442

13 Martinez ME et al. Physical activity, body mass index, and prostaglandin E2 levels in rectal mucosa. J Natl Cancer Inst 1999; 91 (11): 950 - 953

14 Giovannucci E. Modifiable risk factors for colon cancer. Gastroenterol Clin North Am 2002; 31 (4): 925 - 943

15 Giovannucci E. Diet, body weight, and colorectal cancer: a summary of the epidemiologic evidence. J Womens Health (Larchmt) 2003; 12 (2): $173-182$

16 Renehan AG et al. Body-mass index and incidence of cancer: a systematic review and meta-analysis of prospective observational studies. Lancet 2008; 371 (9612): 569 - 578

17 Pischon $T$ et al. Body size and risk of colon and rectal cancer in the European Prospective Investigation Into Cancer and Nutrition (EPIC). J Natl Cancer Inst 2006; 98 (13): 920 - 931

18 Ning Y, Wang L, Giovannucci EL. A quantitative analysis of body mass index and colorectal cancer: findings from 56 observational studies. Obes Rev 2010; 11 (1): 19-30

19 Terry $M B$, Neugut AI. Cigarette smoking and the colorectal adenomacarcinoma sequence: a hypothesis to explain the paradox. Am J Epidemiol 1998; 147 (10): 903 - 910

20 Almendingen $\mathrm{K}$ et al. Smoking and colorectal adenomas: a case-control study. Eur J Cancer Prev 2000; 9 (3): 193-203

21 Chao A et al. Cigarette smoking and colorectal cancer mortality in the cancer prevention study II. J Natl Cancer Inst 2000; 92 (23): 1888 1896

22 Giovannucci E. An updated review of the epidemiological evidence that cigarette smoking increases risk of colorectal cancer. Cancer Epidemiol Biomarkers Prev 2001; 10 (7): 725 - 731

23 Reid ME et al. Smoking exposure as a risk factor for prevalent and recurrent colorectal adenomas. Cancer Epidemiol Biomarkers Prev 2003 ; 12 (10): $1006-1011$

24 Botteri E et al. Cigarette smoking and adenomatous polyps: a metaanalysis. Gastroenterology 2008; 134 (2): 388 - 395

25 Botteri $E$ et al. Smoking and colorectal cancer: a meta-analysis. JAMA 2008; 300 (23): $2765-2778$

26 Miller PE et al. Dietary patterns and colorectal adenoma and cancer risk: a review of the epidemiological evidence. Nutr Cancer 2010; 62 (4): $413-424$

27 Cottet $V$ et al. Dietary patterns and the risk of colorectal adenoma recurrence in a European intervention trial. Eur J Cancer Prev 2005; 14 (1): $21-29$

28 Mai $\mathrm{V}$ et al. Diet quality and subsequent cancer incidence and mortality in a prospective cohort of women. Int J Epidemiol 2005; 34 (1): 54-60

29 Sansbury $L B$ et al. The effect of strict adherence to a high-fiber, highfruit and -vegetable, and low-fat eating pattern on adenoma recurrence. Am J Epidemiol 2009; 170 (5): $576-584$

30 Wakai $\mathrm{K}$ et al. Dietary risk factors for colon and rectal cancers: a comparative case-control study. J Epidemiol 2006; 16 (3): 125-135

31 Beresford SA et al. Low-fat dietary pattern and risk of colorectal cancer: the Women's Health Initiative Randomized Controlled Dietary Modification Trial. JAMA 2006; 295 (6): 643-654

32 Lanza $E$ et al. The polyp prevention trial continued follow-up study: no effect of a low-fat, high-fiber, high-fruit, and -vegetable diet on adenoma recurrence eight years after randomization. Cancer Epidemiol Biomarkers Prev 2007; 16 (9): 1745 - 1752

33 Dahm CC et al. Dietary fiber and colorectal cancer risk: a nested casecontrol study using food diaries. J Natl Cancer Inst 2010; 102 (9): 614 626 
34 Ishikawa $\mathrm{H}$ et al. Randomized trial of dietary fiber and Lactobacillus casei administration for prevention of colorectal tumors. Int J Cancer 2005; 116 (5): 762 - 767

35 Jacobs ET et al. Fiber, sex, and colorectal adenoma: results of a pooled analysis. Am J Clin Nutr 2006; 83 (2): 343-349

36 Park Y et al. Dietary fiber intake and risk of colorectal cancer: a pooled analysis of prospective cohort studies. JAMA 2005; 294 (22): 2849 2857

37 Robertson DJ et al. Fat, fiber, meat and the risk of colorectal adenomas. Am J Gastroenterol 2005; 100 (12): 2789-2795

38 Austin GL et al. Moderate alcohol consumption protects against colorectal adenomas in smokers. Dig Dis Sci 2008; 53 (1): 116 - 122

39 Cho E et al. Alcohol intake and colorectal cancer: a pooled analysis of 8 cohort studies. Ann Intern Med 2004; 140 (8): $603-613$

40 Mizoue $T$ et al. Alcohol drinking and colorectal cancer in Japanese: a pooled analysis of results from five cohort studies. Am J Epidemiol 2008; 167 (12): $1397-1406$

41 Moskal A et al. Alcohol intake and colorectal cancer risk: a dose-response meta-analysis of published cohort studies. Int J Cancer 2007; 120 (3): $664-671$

42 Chao A et al. Meat consumption and risk of colorectal cancer. JAMA 2005; 293 (2): 172-182

43 Larsson SC, Wolk A. Meat consumption and risk of colorectal cancer: a meta-analysis of prospective studies. Int J Cancer 2006; 119 (11): $2657-2664$

44 Mathew A et al. Meat intake and the recurrence of colorectal adenomas. Eur J Cancer Prev 2004; 13 (3): 159-164

45 Norat $T$ et al. Meat, fish, and colorectal cancer risk: the European Prospective Investigation into cancer and nutrition. J Natl Cancer Inst 2005; 97 (12): $906-916$

46 Sinha $R$ et al. Meat, meat cooking methods and preservation, and risk for colorectal adenoma. Cancer Res 2005; 65 (17): 8034 - 8041

47 Geelen A et al. Fish consumption, n-3 fatty acids, and colorectal cancer: a meta-analysis of prospective cohort studies. Am J Epidemiol 2007; 166 (10): $1116-1125$

48 Sugawara $Y$ et al. Fish consumption and the risk of colorectal cancer: the Ohsaki Cohort Study. Br J Cancer 2009; 101 (5): 849 - 854

49 Pot GK et al. Fish consumption and markers of colorectal cancer risk: a multicenter randomized controlled trial. Am J Clin Nutr 2009; 90 (2): $354-361$

50 Je Y, Liu W, Giovannucci E. Coffee consumption and risk of colorectal cancer: a systematic review and meta-analysis of prospective cohort studies. Int J Cancer 2009; 124 (7): 1662 - 1668

51 Sun CL et al. Green tea, black tea and colorectal cancer risk: a meta-analysis of epidemiologic studies. Carcinogenesis 2006; 27 (7): 1301 1309

52 Zhang X et al. Risk of colon cancer and coffee, tea, and sugar-sweetened soft drink intake: pooled analysis of prospective cohort studies. J Natl Cancer Inst 2010; 102 (11): $771-783$

53 Foschi $R$ et al. Citrus fruit and cancer risk in a network of case-control studies. Cancer Causes Control 2010; 21 (2): 237-242

54 Millen AE et al. Fruit and vegetable intake and prevalence of colorectal adenoma in a cancer screening trial. Am J Clin Nutr 2007; 86 (6): $1754-1764$

55 Koushik A et al. Fruits, vegetables, and colon cancer risk in a pooled analysis of 14 cohort studies. J Natl Cancer Inst 2007; 99 (19): 1471 1483

56 Limburg PJ et al. Prospective evaluation of trans-fatty acid intake and colorectal cancer risk in the Iowa Women's Health Study. Int J Cancer 2008; 123 (11): $2717-2719$

57 Alexander DD et al. Meta-analysis of animal fat or animal protein intake and colorectal cancer. Am J Clin Nutr 2009; 89 (5): 1402-1409

58 Galeone $C$ et al. Fried foods, olive oil and colorectal cancer. Ann Oncol 2007; 18 (1): $36-39$

59 Nakamura $T$ et al. Excessive fat restriction might promote the recurrence of colorectal tumors. Nutr Cancer 2010; 62 (2): 154-163

60 Larsson SC et al. Dietary acrylamide intake and risk of colorectal cancer in a prospective cohort of men. Eur J Cancer 2009; 45 (4): 513 - 516

61 Hogervorst JG et al. Dietary acrylamide intake is not associated with gastrointestinal cancer risk. J Nutr 2008; 138 (11): 2229 - 2236

62 Mucci LA, Wilson KM. Acrylamide intake through diet and human cancer risk. J Agric Food Chem 2008; 56 (15): 6013 - 6019

63 Mucci LA, Adami HO, Wolk A. Prospective study of dietary acrylamide and risk of colorectal cancer among women. Int J Cancer 2006; 118 (1): $169-173$
64 Bjelakovic $G$ et al. Mortality in randomized trials of antioxidant supplements for primary and secondary prevention: systematic review and meta-analysis. JAMA 2007; 297 (8): 842 - 857

65 Weingarten MA, Zalmanovici A, Yaphe J. Dietary calcium supplementation for preventing colorectal cancer and adenomatous polyps. Cochrane Database Syst Rev 2008; 1: CD003548

66 Wactawski-Wende J et al. Calcium plus vitamin D supplementation and the risk of colorectal cancer. N Engl J Med 2006; 354 (7): 684-696

67 Sanjoaquin MA et al. Folate intake and colorectal cancer risk: a metaanalytical approach. Int J Cancer 2005; 113 (5): 825 - 828

$68 \mathrm{Wu} \mathrm{Ket}$ al. A randomized trial on folic acid supplementation and risk of recurrent colorectal adenoma. Am J Clin Nutr 2009; 90 (6): 1623 1631

69 Jaszewski $R$ et al. Folic acid supplementation inhibits recurrence of colorectal adenomas: a randomized chemoprevention trial. World J Gastroenterol 2008; 14 (28): $4492-4498$

70 Cole BF et al. Folic acid for the prevention of colorectal adenomas: a randomized clinical trial. JAMA 2007; 297 (21): 2351 - 2359

71 Logan RF et al. Aspirin and folic acid for the prevention of recurrent colorectal adenomas. Gastroenterology 2008; 134 (1): 29-38

72 Grau MV et al. Prolonged effect of calcium supplementation on risk of colorectal adenomas in a randomized trial. J Natl Cancer Inst 2007; 99 (2): $129-136$

73 Wallace $K$ et al. Effect of calcium supplementation on the risk of large bowel polyps. J Natl Cancer Inst 2004; 96 (12): 921 - 5

74 Hartman TJ et al. The association of calcium and vitamin D with risk of colorectal adenomas. J Nutr 2005; 135 (2): 252 - 259

75 Lin J et al. Total magnesium intake and colorectal cancer incidence in women. Cancer Epidemiol Biomarkers Prev 2006; 15 (10): 2006 - 2009

76 Jacobs ET et al. Selenium and colorectal adenoma: results of a pooled analysis. J Natl Cancer Inst 2004; 96 (22): 1669-1675

77 Peters $U$ et al. High serum selenium and reduced risk of advanced colorectal adenoma in a colorectal cancer early detection program. Cancer Epidemiol Biomarkers Prev 2006; 15 (2): 315 - 320

78 Vinogradova $Y$ et al. Risk of colorectal cancer in patients prescribed statins, nonsteroidal anti-inflammatory drugs, and cyclooxygenase-2 inhibitors: nested case-control study. Gastroenterology 2007; 133 (2): $393-402$

79 Bertagnolli MM et al. Celecoxib for the prevention of sporadic colorectal adenomas. N Engl J Med 2006; 355 (9): 873-884

80 Arber $N$ et al. Celecoxib for the prevention of colorectal adenomatous polyps. N Engl J Med 2006; 355 (9): 885 - 895

81 Baron JA et al. A randomized trial of rofecoxib for the chemoprevention of colorectal adenomas. Gastroenterology 2006; 131 (6): 1674-1682

82 Bardou M, Barkun A, Martel M. Effect of statin therapy on colorectal cancer. Gut 2010; 59 (11): 1572 - 1585

83 Routine aspirin or nonsteroidal anti-inflammatory drugs for the primary prevention of colorectal cancer: U.S. Preventive Services Task Force recommendation statement. Ann Intern Med 2007; 146 (5): $361-364$

84 Flossmann E, Rothwell PM. Effect of aspirin on long-term risk of colorectal cancer: consistent evidence from randomised and observationa studies. Lancet 2007; 369 (9573): 1603 - 1613

85 Rothwell PM et al. Effect of daily aspirin on long-term risk of death due to cancer: analysis of individual patient data from randomised trials. Lancet 2011; 377 (9759): 31 - 41

86 Chan AT et al. Long-term use of aspirin and nonsteroidal anti-inflammatory drugs and risk of colorectal cancer. JAMA 2005; 294 (8): 914 923

87 Hormone therapy for the prevention of chronic conditions in postmenopausal women: recommendations from the U.S. Preventive Services Task Force. Ann Intern Med 2005; 142 (10): 855-860

88 Farquhar $C$ et al. Long term hormone therapy for perimenopausal and postmenopausal women. Cochrane Database Syst Rev 2009; 2: CD004143

89 Alberts DS et al. Phase III trial of ursodeoxycholic acid to prevent colorectal adenoma recurrence. J Natl Cancer Inst 2005; 97 (11): 846 - 853

90 DGGG. Hormontherapie (HT) in der Peri- und Postmenopause; 2009

91 Birkner BR. Evidence-based prevention of colorectal carcinoma. Dtsch Med Wochenschr 2003; 128 (49): 2598-2603

92 Winawer SJ et al. Colorectal cancer screening: clinical guidelines and rationale. Gastroenterology 1997; 112 (2): 594-642

93 Imperiale TF et al. Results of screening colonoscopy among persons 40 to 49 years of age. N Engl J Med 2002; 346 (23): 1781 - 1785 
94 Whitlock EP et al. Screening for colorectal cancer: a targeted, updated systematic review for the U.S. Preventive Services Task Force. Ann Intern Med 2008; 149 (9): 638 - 658

95 Stevens T, Burke CA. Colonoscopy screening in the elderly: when to stop? Am J Gastroenterol 2003; 98 (8): 1881 - 1885

96 Kirchgatterer $A$ et al. Colonoscopy and sigmoidoscopy in patients aged eighty years or older. Z Gastroenterol 2002; 40 (12): 951 - 956

97 Pox CP et al. Efficacy of a Nationwide Screening Colonoscopy Program for Colorectal Cancer. Gastroenterology 2012; 142: 1460 - 1467

98 Zhang B, Fattah A, Nakama H. Characteristics and survival rate of elderly patients with colorectal cancer detected by immunochemical occult blood screening. Hepatogastroenterology 2000; 47 (32): 414-418

99 Winawer SJ et al. Prevention of colorectal cancer by colonoscopic polypectomy. The National Polyp Study Workgroup. N Engl J Med 1993; 329 (27): 1977 - 1981

100 Citarda $F$ et al. Efficacy in standard clinical practice of colonoscopic polypectomy in reducing colorectal cancer incidence. Gut 2001; 48 (6): $812-815$

101 Zauber AG et al. Colonoscopic polypectomy and long-term prevention of colorectal-cancer deaths. N Engl J Med 2012; 366 (8): 687 -696

$102 \mathrm{Hol} \mathrm{L}$ et al. Screening for colorectal cancer: randomised trial comparing guaiac-based and immunochemical faecal occult blood testing and flexible sigmoidoscopy. Gut 2010; 59 (1): 62 -68

103 Quintero E et al. Colonoscopy versus fecal immunochemical testing in colorectal-cancer screening. N Engl J Med 2012; 366 (8): 697 - 706

104 Bekanntmachungen: Richtlinien des Bundesausschusses der Ärzte und Krankenkassen über die Früherkennung von Krebserkrankungen („Krebsfrüherkennungs-Richtlinien“). Dtsch Arztebl Int 2002; 1 (11): 518

105 Huppe D et al. Effectiveness of screening colonoscopy in a communitybased study. Z Gastroenterol 2008; 46 (2): 193-200

106 Kahi CJ et al. Effect of screening colonoscopy on colorectal cancer incidence and mortality. Clin Gastroenterol Hepatol 2009; 7 (7): 770 775 quiz 711

107 Imperiale TF et al. Risk of advanced proximal neoplasms in asymptomatic adults according to the distal colorectal findings. N Engl J Med 2000; 343 (3): $169-174$

108 Lieberman DA et al. Use of colonoscopy to screen asymptomatic adults for colorectal cancer. Veterans Affairs Cooperative Study Group 380. N Engl J Med 2000; 343 (3): 162 - 168

109 Atkin $W$ et al. Single flexible sigmoidoscopy screening to prevent colorectal cancer: baseline findings of a UK multicentre randomised trial. Lancet 2002; 359 (9314): $1291-1300$

110 Muller AD, Sonnenberg A. Protection by endoscopy against death from colorectal cancer. A case-control study among veterans. Arch Intern Med 1995; 155 (16): 1741 - 1748

111 Newcomb PA et al. Screening sigmoidoscopy and colorectal cancer mortality. J Natl Cancer Inst 1992; 84 (20): 1572 - 1575

112 Selby JV et al. A case-control study of screening sigmoidoscopy and mortality from colorectal cancer. N Engl J Med 1992; 326 (10): 653 657

113 Singh $\mathrm{H}$ et al. Predictors of colorectal cancer after negative colonoscopy: a population-based study. Am J Gastroenterol 2010; 105 (3): 663-673; quiz 674

114 Brenner $H$ et al. Protection from right- and left-sided colorectal neoplasms after colonoscopy: population-based study. J Natl Cancer Inst 2010; 102 (2): $89-95$

115 Brenner $H$ et al. Protection from colorectal cancer after colonoscopy: a population-based, case-control study. Ann Intern Med 2011; 154 (1): $22-30$

116 Sieg A, Hachmoeller-Eisenbach U, Eisenbach T. Prospective evaluation of complications in outpatient GI endoscopy: a survey among German gastroenterologists. Gastrointest Endosc 2001; 53: 620-627

117 Rex DK et al. Colonoscopic miss rates of adenomas determined by back-to-back colonoscopies. Gastroenterology 1997; 112 (1): 24-28

118 Rex DK et al. 5-year incidence of adenomas after negative colonoscopy in asymptomatic average-risk persons [see comment]. Gastroenterology 1996; 111 (5): $1178-1181$

119 Brenner $H$ et al. Long-term risk of colorectal cancer after negative colonoscopy. J Clin Oncol 2011; 29 (28): 3761 - 3767

120 Bundesärztekammer: Richtlinien zur prädiktiven genetischen Diagnostik. Dt Ärztebl 2003; 100 (19): A1297 - 1305

121 Atkin WS et al. Once-only flexible sigmoidoscopy screening in prevention of colorectal cancer: a multicentre randomised controlled trial. Lancet 2010; 375 (9726): 1624-1633
122 Muller AD, Sonnenberg A. Prevention of colorectal cancer by flexible endoscopy and polypectomy. A case-control study of 32,702 veterans. Ann Intern Med 1995; 123 (12): 904 -910

123 Newcomb PA et al. Long-term efficacy of sigmoidoscopy in the reduction of colorectal cancer incidence. J Natl Cancer Inst 2003; 95 (8): $622-625$

124 Schoen RE et al. Results of repeat sigmoidoscopy 3 years after a negative examination. Jama 2003; 290 (1): 41 - 48

125 Burke CA, Elder K, Lopez R. Screening for colorectal cancer with flexible sigmoidoscopy: is a 5-yr interval appropriate? A comparison of the detection of neoplasia 3 yr versus $5 \mathrm{yr}$ after a normal examination. Am J Gastroenterol 2006; 101 (6): 1329-1332

126 Winawer SJ et al. Screening for colorectal cancer with fecal occult blood testing and sigmoidoscopy. J Natl Cancer Inst 1993; 85 (16): $1311-1318$

127 Lieberman DA, Weiss DG. One-time screening for colorectal cancer with combined fecal occult-blood testing and examination of the distal colon. N Engl J Med 2001; 345 (8): 555-560

128 Gondal $G$ et al. The Norwegian Colorectal Cancer Prevention (NORCCAP) screening study: baseline findings and implementations for clinical work-up in age groups 50-64 years. Scand J Gastroenterol 2003; 38 (6): $635-642$

129 Kato J et al. Combination of sigmoidoscopy and a fecal immunochemical test to detect proximal colon neoplasia. Clin Gastroenterol Hepatol 2009; 7 (12): 1341 - 1346

130 Eliakim $R$ et al. Evaluation of the PillCam Colon capsule in the detection of colonic pathology: results of the first multicenter, prospective, comparative study. Endoscopy 2006; 38 (10): $963-70$

131 Schoofs N, Deviere J, Van Gossum A. PillCam colon capsule endoscopy compared with colonoscopy for colorectal tumor diagnosis: a prospective pilot study. Endoscopy 2006; 38 (10): 971 - 977

132 Van Gossum A et al. Capsule endoscopy versus colonoscopy for the detection of polyps and cancer. N Engl J Med 2009; 361 (3): 264-270

133 Spada C et al. Meta-analysis shows colon capsule endoscopy is effective in detecting colorectal polyps. Clin Gastroenterol Hepatol 2010; 8 (6): $516-522$

134 Gay G et al. Could the colonic capsule PillCam Colon be clinically useful for selecting patients who deserve a complete colonoscopy? Results of clinical comparison with colonoscopy in the perspective of colorectal cancer screening. Am J Gastroenterol 2010; 105 (5): $1076-1086$

135 Eliakim $R$ et al. Prospective multicenter performance evaluation of the second-generation colon capsule compared with colonoscopy. Endoscopy 2009; 41 (12): 1026-1031

136 Spada $C$ et al. Second-generation colon capsule endoscopy compared with colonoscopy. Gastrointest Endosc 2011; 74 (3): 581 - 589 e1

137 Mandel JS et al. Reducing mortality from colorectal cancer by screening for fecal occult blood. Minnesota Colon Cancer Control Study. N Engl J Med 1993; 328 (19): 1365 - 1371

138 Zhu MM et al. Comparison of immunochemical and guaiac-based fecal occult blood test in screening and surveillance for advanced colorectal neoplasms: a meta-analysis. J Dig Dis 2010; 11 (3): 148-160

139 Rossum LG et al. Colorectal cancer screening comparing no screening, immunochemical and guaiac fecal occult blood tests: a cost-effectiveness analysis. Int J Cancer 2011; 128 (8): 1908 - 1917

140 Hundt S, Haug $U$, Brenner H. Comparative evaluation of immunochemical fecal occult blood tests for colorectal adenoma detection. Ann Intern Med 2009; 150 (3): 162 - 169

141 Brenner $H$, Haug $U$, Hundt $S$. Inter-test agreement and quantitative cross-validation of immunochromatographical fecal occult blood tests. Int J Cancer 2010; 127 (7): 1643 - 1649

142 Haug $U$, Hundt S, Brenner $H$. Quantitative immunochemical fecal occult blood testing for colorectal adenoma detection: evaluation in the target population of screening and comparison with qualitative tests. Am J Gastroenterol 2010; 105 (3): $682-690$

143 Allison JE et al. Screening for colorectal neoplasms with new fecal occult blood tests: update on performance characteristics. J Natl Cancer Inst 2007; 99 (19): 1462 - 1470

144 Park DI et al. Comparison of guaiac-based and quantitative immunochemical fecal occult blood testing in a population at average risk undergoing colorectal cancer screening. Am J Gastroenterol 2010; 105 (9): 2017-2025

145 Launoy GD et al. Evaluation of an immunochemical fecal occult blood test with automated reading in screening for colorectal cancer in a general average-risk population. Int J Cancer 2005; 115 (3): 493 - 496 
146 Roon AH et al. Diagnostic yield improves with collection of 2 samples in fecal immunochemical test screening without affecting attendance. Clin Gastroenterol Hepatol 2011; 9 (4): 333 - 339

147 Doran J, Hardcastle JD. Bleeding patterns in colorectal cancer: the effect of aspirin and the implications for faecal occult blood testing. Br J Surg 1982; 69 (12): 711 - 713

148 Ahlquist DA et al. Patterns of occult bleeding in asymptomatic colorectal cancer. Cancer 1989; 63 (9): 1826-1830

149 Macrae FA, St JohnDJ. Relationship between patterns of bleeding and hemoccult sensitivity in patients with colorectal cancers or adenomas. Gastroenterology 1982; 82: 891 - 898

150 Greegor $\mathrm{DH}$. Occult blood testing for detection of asymptomatic colon cancer. Cancer 1971; 28: 131 - 134

151 Hardcastle JD et al. Randomised controlled trial of faecal-occult-blood screening for colorectal cancer. Lancet 1996; 348 (9040): 1472 - 1477

152 Kronborg 0 et al. Randomised study of screening for colorectal cancer with faecal-occult-blood test. Lancet 1996; 348 (9040): 1467-1471

153 Hewitson $P$ et al. Screening for colorectal cancer using the faecal occult blood test, Hemoccult. Cochrane Database Syst Rev 2007; 1: CD001216

154 Levin B, Hess K, Johnson C et al. A comparison of 3 fecal occult blood tests. Arch Intern Med 1997; 157 (9): 970 - 976

155 Gnauck R, Macrae FA, Fleisher M. How to perform the fecal occult blood test. CA Cancer J Clin 1984; 34: 134-147

156 Jaffe RM et al. False-negative stool occult blood tests caused by ingestion of ascorbic acid (vitamin C). Ann Intern Med 1975; 83 (6): 824 826

157 Macrae FA et al. Optimal dietary conditions for hemoccult testing. Gastroenterology 1982; 82 (5): 899-903

158 Rozen P, Knaani J, Samuel Z. Eliminating the need for dietary restrictions when using a sensitive guaiac fecal occult blood test. Dig Dis Sci 1999; 44 (4): $756-760$

159 Pignone $M$ et al. Meta-analysis of dietary restriction during fecal occult blood testing. Eff Clin Pract 2001; 4 (4): 150 - 156

160 Rossum LG et al. Random comparison of guaiac and immunochemical fecal occult blood tests for colorectal cancer in a screening population. Gastroenterology 2008; 135 (1): 82 -90

161 Webendorfer $S$ et al. Precautions for intestinal cancer in the workplace. An initiative for secondary prevention in the BASF joint-stock company. Dtsch Med Wochenschr 2004; 129 (6): 239-243

162 Mandel JS et al. The effect of fecal occult-blood screening on the incidence of colorectal cancer. N Engl J Med 2000; 343 (22): 1603-1607

163 Traverso $G$ et al. Detection of APC mutations in fecal DNA from patients with colorectal tumors. N Engl J Med 2002; 346 (5): 311 - 320

164 Ahlquist DA et al. Colorectal cancer screening by detection of altered human DNA in stool: feasibility of a multitarget assay panel. Gastroenterology 2000; 119 (5): 1219-1227

165 Dong SM et al. Detecting colorectal cancer in stool with the use of multiple genetic targets. J Natl Cancer Inst 2001; 93 (11): 858 - 865

166 Tagore KS et al. Sensitivity and specificity of a stool DNA multitarget assay panel for the detection of advanced colorectal neoplasia. Clin Colorectal Cancer 2003; 3 (1): 47 - 53

167 Imperiale TF et al. Fecal DNA versus fecal occult blood for colorectalcancer screening in an average-risk population. N Engl J Med 2004; 351 (26): $2704-2714$

168 Ahlquist DA et al. Stool DNA and occult blood testing for screen detection of colorectal neoplasia. Ann Intern Med 2008; 149 (7): 441 450, W81

169 Haug $U$, Hundt S, Brenner $H$. Sensitivity and specificity of faecal tumour M2 pyruvate kinase for detection of colorectal adenomas in a large screening study. Br J Cancer 2008; 99 (1): 133 -135

170 Moslein $G$ et al. Analysis of the statistical value of various commercially available stool tests - a comparison of one stool sample in correlation to colonoscopy. Dtsch Med Wochenschr 2010; 135 (12): 557 562

171 Haan MC et al. Diagnostic value of CT-colonography as compared to colonoscopy in an asymptomatic screening population: a meta-analysis. Eur Radiol 2011; 21 (8): 1747 - 1763

172 El-Maraghi RH, Kielar AZ. CT colonography versus optical colonoscopy for screening asymptomatic patients for colorectal cancer a patient, intervention, comparison, outcome (PICO) analysis. Acad Radiol 2009; 16 (5): $564-571$

173 Pox CP, Schmiegel $W$. Role of CT colonography in colorectal cancer screening: risks and benefits. Gut 2010; 59 (5): 692 - 700
174 Frazier AL et al. Cost-effectiveness of screening for colorectal cancer in the general population. Jama 2000; 284 (15): 1954-1961

175 Geul KW et al. Prevention of colorectal cancer. Costs and effectiveness of sigmoidoscopy. Scand J Gastroenterol Suppl 1997; 223: 79-87

176 Inadomi JM. Update on the cost-effectiveness of screening for colorectal neoplasia. Curr Opin Gastroenterol 2003; 29: 44-50

177 Khandker RK et al. A decision model and cost-effectiveness analysis of colorectal cancer screening and surveillance guidelines for averagerisk adults. Int J Technol Assess Health Care 2000; 16 (3): 799 - 810

178 McGrath JS, Ponich TP, Gregor JC. Screening for colorectal cancer: the cost to find an advanced adenoma. Am J Gastroenterol 2002; 97 (11): $2902-2907$

179 Norum J. Prevention of colorectal cancer: a cost-effectiveness approach to a screening model employing sigmoidoscopy. Ann Oncol 1998; 9 (6): $613-618$

180 Pignone $M$ et al. Cost-effectiveness analyses of colorectal cancer screening: a systematic review for the U.S. Preventive Services Task Force. Ann Intern Med 2002; 137 (2): 96-104

181 Sonnenberg A, Delco F. Cost-effectiveness of a single colonoscopy in screening for colorectal cancer. Arch Intern Med 2002; 162 (2): $163-168$

182 Vijan S et al. Which colon cancer screening test? A comparison of costs, effectiveness, and compliance. Am J Med 2001; 111 (8): 593 601

183 Zauber AG et al. Evaluating test strategies for colorectal cancer screening: a decision analysis for the U.S. Preventive Services Task Force. Ann Intern Med 2008; 149 (9): 659 - 669

184 Lansdorp-Vogelaar I, Knudsen AB, Brenner $H$. Cost-effectiveness of colorectal cancer screening. Epidemiol Rev 2011; 33 (1): 88-100

185 Sung JJ et al. Asia Pacific consensus recommendations for colorectal cancer screening. Gut 2008; 57 (8): 1166-1176

186 Jasperson KW et al. Hereditary and familial colon cancer. Gastroenterology 2010; 138 (6): 2044-2058

187 Andrieu $N$ et al. Familial relative risk of colorectal cancer: a population-based study. Eur J Cancer 2003; 39 (13): 1904-1911

188 Johns LE, Houlston RS. A systematic review and meta-analysis of familial colorectal cancer risk. Am J Gastroenterol 2001; 96 (10): 2992 3003

189 Lichtenstein P et al. Environmental and heritable factors in the causation of cancer - analyses of cohorts of twins from Sweden, Denmark and Finland. N Engl J Med 2000; 343 (2): 78 - 85

190 Slattery ML et al. Family history and colorectal cancer: predictors of risk. Cancer Causes Control 2003; 14 (9): 879-887

191 Winawer $S$ et al. Colorectal cancer screening and surveillance: clinical guidelines and rationale-Update based on new evidence. Gastroenterology 2003; 124 (2): $544-560$

192 Bonelli $L$ et al. Family history of colorectal cancer as a risk factor for benign and malignant tumours of the large bowel. A case-control study. Int J Cancer 1988; 41 (4): 513-517

193 Fuchs CS et al. A prospective study of family history and the risk of colorectal cancer. N Engl J Med 1994; 331 (25): 1669-1674

194 Guillem JG et al. Clustering of colorectal cancer in families of probands under 40 years of age. Dis Colon Rectum 1996; 39 (9): 1004-1007

195 Guillem JG et al. Colonoscopic screening for neoplasms in asymptomatic first-degree relatives of colon cancer patients. A controlled, prospective study. Dis Colon Rectum 1992; 35 (6): 523 -9

196 Kune GA, Kune S, Watson LF. The role of heredity in the etiology of large bowel cancer: data from the Melbourne Colorectal Cancer Study. World J Surg 1989; 13 (1): 124-129

197 Rozen Pet al. Family history of colorectal cancer as a marker of potential malignancy within a screening programm. Cancer 1987; 60 (2): $248-254$

198 Sondergaard JO, Bulow S, Lynge E. Cancer incidence among parents of patients with colorectal cancer. Int J Cancer 1991; 47 (2): 202 - 206

199 St John DJ et al. Cancer risk in relatives of patients with common colorectal cancer. Ann Int Med 1993; 118 (10): 785 - 790

200 Butterworth AS, Higgins JP, Pharoah P. Relative and absolute risk of colorectal cancer for individuals with a family history: a meta-analysis. Eur J Cancer 2006; 42 (2): 216-227

201 Hall NR et al. Hereditary susceptibility to colorectal cancer. Relatives of early onset cases are particularly at risk. Dis Colon Rectum 1996; 39 (7): $739-743$

202 Hunt LM et al. Endoscopic screening of relatives of patients with colorectal cancer. Gut 1998; 42 (1): 71 - 75 
203 Winawer SJ et al. Risk of colorectal cancer in the families of patients with adenomatous polyps. National Polyp Study Workgroup. N Engl J Med 1996; 334 (2): $82-87$

204 Ahsan $\mathrm{H}$ et al. Family history of colorectal adenomatous polyps and increased risk for colorectal cancer. Ann Intern Med 1998; 128 (11): 900-905

205 Nakama $\mathrm{H}$ et al. Family history of colorectal adenomatous polyps as a risk factor for colorectal cancer. Eur J Cancer 2000; 36 (16): 2111 2114

206 Almendingen K, Hofstad B, Vatn MH. Does a family history of cancer increase the risk of occurrence, growth, and recurrence of colorectal adenomas? Gut 2003; 52 (5): $747-751$

207 Winawer SJ et al. Risk and surveillance of individuals with colorectal polyps. WHO Collaborating Centre for the Prevention of Colorectal Cancer. Bull World Health Organ 1990; 68 (6): 789 - 795

208 Atkin WS, Morson BC, Cuzick J. Long-term risk of colorectal cancer after excision of rectosigmoid adenomas. N Engl J Med 1992; 326 (10): 658-662

209 Otchy DP et al. Metachronous colon cancer in persons who have had a large adenomatous polyp. Am J Gastroenterol 1996; 91 (3): 448 - 454

210 Stolck $R U$ et al. Adenoma characteristics at first colonoscopy as predictors of adenoma recurrence and characteristics at follow-up. The Polyp Prevention Study Group. Gastroenterology 1998; 115 (1): $13-18$

211 Noshirwani KC et al. Adenoma size and number are predictive of adenoma recurrence: implications for surveillance colonoscopy. Gastrointest Endosc 2000; 51: $433-437$

212 Aldridge AJ, Simson JN. Histological assessment of colorectal adenomas by size. Are polyps less than $10 \mathrm{~mm}$ in size clinically important? Eur J Surg 2001; 167 (10): 777 - 781

213 Avidan $B$ et al. New occurrence and recurrence of neoplasms within 5 years of a screening colonoscopy. Am J Gastroenterol 2002; 97 (6): $1524-1529$

214 Bertario $L$ et al. Predictors of metachronous colorectal neoplasms in sporadic adenoma patients. Int J Cancer 2003; 105 (1): 82 - 87

215 Chen $C D$ et al. A case-cohort study for the disease natural history of adenoma-carcinoma and de novo carcinoma and surveillance of colon and rectum after polypectomy: implication for efficacy of colonoscopy. Br J Cancer 2003; 88 (12): 1866 - 1873

216 Bensen $S$ et al. The colonoscopic miss rate and true one-year recurrence of colorectal neoplastic polyps. Polyp Prevention Study Group. Am J Gastroenterol 1999; 94 (1): 194 -199

217 Katalinic A, Raspe H, Waldmann A. Positive family history of colorectal cancer - use of a questionnaire. Z Gastroenterol 2009; 47 (11): 1125 1131

218 Baron JA et al. Neoplastic and antineoplastic effects of beta-carotene on colorectal adenoma recurrence: results of a randomized trial. J Natl Cancer Inst 2003; 95 (10): 717 - 722

219 Karnes WE. Epidemiology and etiology of sporadic colorectal adenocarcinoma. Current opinion in Gastroenterology 1994; 10: 19-26

220 Sandler $R S$ et al. A randomized trial of aspirin to prevent colorectal adenomas in patients with previous colorectal cancer. N Engl J Med 2003; 348 (10): $883-890$

221 Rothwell PM et al. Long-term effect of aspirin on colorectal cancer incidence and mortality: 20-year follow-up of five randomised trials. Lancet 2010; 376 (9754): 1741 - 1750

222 Burt RW. Screening of patients with a positive family history of colorectal cancer. Gastrointest Endosc Clin N Am 1997; 7 (1): 65 - 79

223 Gilbert JM et al. Feasibility study of colonoscopy as the primary screening investigation in relatives of patients with colorectal cancer. Ann R Coll Surg Engl 2001; 83 (6): 415 - 419

224 Syrigos $K N$ et al. Colonoscopy in asymptomatic individuals with a family history of colorectal cancer. Ann Surg Oncol 2002; 9 (5): $439-443$

225 Bradshaw $N$ et al. Colonoscopy surveillance of individuals at risk of familial colorectal cancer. Gut 2003; 52 (12): 1748 - 1751

226 Brenner H, Hoffmeister M, Haug U. Family history and age at initiation of colorectal cancer screening. Am J Gastroenterol 2008; 103 (9): 2326- 2331

227 Waldmann A, Raspe H, Katalinic A. Colon cancer risk in persons at familial or hereditary risk aged < 55 years. Z Gastroenterol 2009; 47 (10): $1052-1058$

228 Levin $B$ et al. Screening and surveillance for the early detection of colorectal cancer and adenomatous polyps, 2008: a joint guideline from the American Cancer Society, the US Multi-Society Task Force on Colorectal Cancer, and the American College of Radiology. Gastroenterology 2008; 134 (5): 1570-1595

229 Engel $C$ et al. Efficacy of annual colonoscopic surveillance in individuals with hereditary nonpolyposis colorectal cancer. Clin Gastroenterol Hepatol 2010; 8 (2): 174-182

230 Dove-Edwin I et al. Prevention of colorectal cancer by colonoscopic surveillance in individuals with a family history of colorectal cancer: 16 year, prospective, follow-up study. BMJ 2005; 331 (7524): 1047

231 Duncan RE et al. The challenge of developmentally appropriate care: predictive genetic testing in young people for familial adenomatous polyposis. Fam Cancer 2010; 9 (1): 27 - 35

232 Douma KF et al. Psychosocial issues in genetic testing for familial adenomatous polyposis: a review of the literature. Psychooncology 2008; 17 (8): $737-745$

233 Douma KF et al. Psychological distress and use of psychosocial support in familial adenomatous polyposis. Psychooncology 2010; 19 (3): $289-298$

234 Vasen HF et al. The International Collaborative Group on Hereditary Non-Polyposis Colorectal Cancer (ICG-HNPCC). Dis Colon Rectum $1991 ; 34$ (5): $424-425$

235 Rodriguez-Bigas MA et al. A National Cancer Institute Workshop on Hereditary Nonpolyposis Colorectal Cancer Syndrome: meeting highlights and Bethesda guidelines. J Natl Cancer Inst 1997; 89 (23): $1758-1762$

236 Schulmann $\mathrm{K}$ et al. Small bowel cancer risk in Lynch syndrome. Gut 2008; 57 (11): 1629-1630

237 Kate GL et al. Is surveillance of the small bowel indicated for Lynch syndrome families? Gut 2007; 56 (9): 1198-1201

238 Schulmann Ket al. HNPCC-associated small bowel cancer: clinical and molecular characteristics. Gastroenterology 2005; 128 (3): 590 - 599

239 der Post RS et al. Risk of urothelial bladder cancer in Lynch syndrome is increased, in particular among MSH2 mutation carriers. J Med Genet 2010; 47 (7): 464-470

240 Kastrinos F et al. Risk of pancreatic cancer in families with Lynch syndrome. JAMA 2009; 302 (16): 1790-1795

241 Win AK et al. Colorectal and Other Cancer Risks for Carriers and Noncarriers From Families With a DNA Mismatch Repair Gene Mutation: A Prospective Cohort Study. J Clin Oncol 2012; 30: 958-964

242 Vasen HF et al. MSH2 mutation carriers are at higher risk of cancer than MLH1 mutation carriers: a study of hereditary nonpolyposis colorectal cancer families. J Clin Oncol 2001; 19 (20): 4074-4080

243 Aarnio $M$ et al. Life-time risk of different cancers in hereditary nonpolyposis colorectal cancer (HNPCC) syndrome. Int J Cancer 1995; 64 (6): $430-433$

244 Vasen HF et al. The risk of brain tumours in hereditary non-polyposis colorectal cancer (HNPCC). Int J Cancer 1996; 65 (4): 422-425

245 Kruse $R$ et al. Muir-Torre phenotype has a frequency of DNA mismatch-repair-gene mutations similar to that in hereditary nonpolyposis colorectal cancer families defined by the Amsterdam criteria. Am J Hum Genet 1998; 63 (1): 63 - 70

246 Umar A et al. Revised Bethesda Guidelines for hereditary nonpolyposis colorectal cancer (Lynch syndrome) and microsatellite instability. J Natl Cancer Inst 2004; 96 (4): 261 -268

247 Shia J. Immunohistochemistry versus microsatellite instability testing for screening colorectal cancer patients at risk for hereditary nonpolyposis colorectal cancer syndrome. Part I. The utility of immunohistochemistry. J Mol Diagn 2008; 10 (4): 293 - 300

248 Deng $G$ et al. BRAF mutation is frequently present in sporadic colorectal cancer with methylated hMLH1, but not in hereditary nonpolyposis colorectal cancer. Clin Cancer Res 2004; 10 (1): 191 - 195

249 Domingo $E$ et al. BRAF screening as a low-cost effective strategy for simplifying HNPCC genetic testing. J Med Genet 2004; 41 (9): 664668

250 Loughrey $M B$ et al. Incorporation of somatic BRAF mutation testing into an algorithm for the investigation of hereditary non-polyposis colorectal cancer. Fam Cancer 2007; 6 (3): 301 - 310

251 McGivern A et al. Promoter hypermethylation frequency and BRAF mutations distinguish hereditary non-polyposis colon cancer from sporadic MSI-H colon cancer. Fam Cancer 2004; 3 (2): 101 - 107

252 Levine AJ et al. Cancer risks for the relatives of colorectal cancer cases with a methylated MLH1 promoter region: data from the Colorectal Cancer Family Registry. Cancer Prev Res (Phila) 2012; 5 (2): 328 - 335

253 Foulkes WD. A tale of four syndromes: familial adenomatous polyposis, Gardner syndrome, attenuated APC and Turcot syndrome. QJM 1995; 88 (12): $853-863$ 
254 Lynch HT et al. Attenuated familial adenomatous polyposis (AFAP). A phenotypically and genotypically distinctive variant of FAP. Cancer 1995; 76 (12): $2427-2433$

255 Lynch HT, Smyrk TC. Classification of familial adenomatous polyposis: a diagnostic nightmare. Am J Hum Genet 1998; 62 (6): 1288 - 1289

256 Soravia $C$ et al. Genotype-phenotype correlations in attenuated adenomatous polyposis coli. Am J Hum Genet 1998; 62: 1290 - 1301

257 Hernegger GS, Moore HG, Guillem JG. Attenuated familial adenomatous polyposis: an evolving and poorly understood entity. Dis Colon Rectum 2002; 45 (1): 127-134; discussion 134-136

258 Knudsen AL, Bisgaard ML, Bulow S. Attenuated familial adenomatous polyposis (AFAP). A review of the literature. Fam Cancer 2003; 2 (1): $43-55$

$259 \mathrm{Al}$-Tassan $\mathrm{N}$ et al. Inherited variants of MYH associated with somatic $\mathrm{G}: \mathrm{C} \rightarrow \mathrm{T}:$ A mutations in colorectal tumors. Nat Genet 2002; 30 (2): $227-232$

$260 \mathrm{Cao} Y$ et al. Challenge in the differentiation between attenuated familial adenomatous polyposis and hereditary nonpolyposis colorectal cancer: case report with review of the literature. Am J Gastroenterol 2002; 97 (7): 1822 - 1827

261 Aretz S. The differential diagnosis and surveillance of hereditary gastrointestinal polyposis syndromes. Dtsch Arztebl Int 2010; 107 (10): $163-173$

262 Aretz S, Genuardi M, Hes FJ. Clinical utility gene card for: MUTYHassociated polyposis (MAP), autosomal recessive colorectal adenomatous polyposis. Eur J Hum Genet 2013; 21: DOI: 10.1038/ ejhg.2012.163

263 Sampson JR, Jones N. MUTYH-associated polyposis. Best Pract Res Clin Gastroenterol 2009; 23 (2): 209-218

264 Morreau H, Riddel R, Aretz S. MUTYH-associated polyposis. In: Bosman FT et al. Editors WHO Classification of Tumours of the Digestive System Lyon: IARC/WHO Press; 2010: 156-159

265 Aretz S et al. MUTYH-associated polyposis: 70 of 71 patients with biallelic mutations present with an attenuated or atypical phenotype. Int J Cancer 2006; 119 (4): 807-814

266 Lubbe SJ et al. Clinical implications of the colorectal cancer risk associated with MUTYH mutation. J Clin Oncol 2009; 27 (24): 3975-3980

267 Cleary SP et al. Germline MutY human homologue mutations and colorectal cancer: a multisite case-control study. Gastroenterology 2009; 136 (4): 1251 - 1260

268 Boparai KS et al. Hyperplastic polyps and sessile serrated adenomas as a phenotypic expression of MYH-associated polyposis. Gastroenterology 2008; 135 (6): 2014-2018

269 Vogt $S$ et al. Expanded extracolonic tumor spectrum in MUTYH-associated polyposis. Gastroenterology 2009; 137 (6): 1976-1985

270 Papadopoulos N, Lindblom A. Molecular basis of HNPCC: mutations of MMR genes. Hum Mut 1997; 10: 89-99

271 Kuiper RP et al. Recurrence and variability of germline EPCAM deletions in Lynch syndrome. Hum Mutat 2011; 32 (4): 407-414

272 Jarvinen $\mathrm{HJ}$ et al. Controlled 15-year trial on screening for colorectal cancer in families with hereditary nonpolyposis colorectal cancer. Gastroenterology 2000; 118 (5): 829-834

273 Vos tot Nederveen Cappel WH et al. Surveillance for hereditary nonpolyposis colorectal cancer: a long-term study on 114 families. Dis Colon Rectum 2002; 45 (12): 1588-1594

274 Edelstein DL et al. Rapid development of colorectal neoplasia in patients with Lynch syndrome. Clin Gastroenterol Hepatol 2011; 9 (4): $340-343$

275 Renkonen-Sinisalo L et al. Surveillance improves survival of colorectal cancer in patients with hereditary nonpolyposis colorectal cancer. Cancer Detect Prev 2000; 24 (2): 137-142

276 Huneburg $R$ et al. Chromocolonoscopy detects more adenomas than white light colonoscopy or narrow band imaging colonoscopy in hereditary nonpolyposis colorectal cancer screening. Endoscopy 2009; 41 (4): $316-322$

277 Stoffel EM et al. Missed adenomas during colonoscopic surveillance in individuals with Lynch Syndrome (hereditary nonpolyposis colorectal cancer). Cancer Prev Res (Phila) 2008; 1 (6): 470 - 475

278 Hurlstone DP et al. High magnification chromoscopic colonoscopy or high frequency $20 \mathrm{MHz}$ mini probe endoscopic ultrasound staging for early colorectal neoplasia: a comparative prospective analysis. Gut 2005; 54 (11): $1585-1589$

279 Aarnio $M$ et al. Cancer risk in mutation carriers of DNA-mismatch-repair genes. Int J Cancer 1999; 81 (2): 214-218
280 Dove-Edwin I et al. The outcome of endometrial carcinoma surveillance by ultrasound scan in women at risk of hereditary nonpolyposis colorectal carcinoma and familial colorectal carcinoma. Cancer 2002; 94 (6): $1708-1712$

281 Rijcken FE et al. Gynecologic screening in hereditary nonpolyposis colorectal cancer. Gynecol Oncol 2003; 91 (1): $74-80$

282 Renkonen-Sinisalo $L$ et al. Surveillance for endometrial cancer in hereditary nonpolyposis colorectal cancer syndrome. Int J Cancer 2007; 120 (4): $821-824$

283 Vasen HF et al. Guidelines for the clinical management of Lynch syndrome (hereditary non-polyposis cancer). J Med Genet 2007; 44 (6): $353-362$

284 Goecke $T$ et al. Genotype-phenotype comparison of German MLH1 and MSH2 mutation carriers clinically affected with Lynch syndrome: a report by the German HNPCC Consortium. J Clin Oncol 2006; 24 (26): $4285-4292$

285 Burn J et al. Effect of aspirin or resistant starch on colorectal neoplasia in the Lynch syndrome. N Engl J Med 2008; 359 (24): 2567-2578

286 Burn J et al. Long-term effect of aspirin on cancer risk in carriers of hereditary colorectal cancer: an analysis from the CAPP2 randomised controlled trial. Lancet 2011; 378 (9809): 2081 -2087

287 Natarajan $N$ et al. Comparison of extended colectomy and limited resection in patients with Lynch syndrome. Dis Colon Rectum 2010; 53 (1): $77-82$

288 Kalady MF et al. Risk of colorectal adenoma and carcinoma after colectomy for colorectal cancer in patients meeting Amsterdam criteria. Ann Surg 2010; 252 (3): 507-511; discussion 511-513

289 Parry S et al. Metachronous colorectal cancer risk for mismatch repair gene mutation carriers: the advantage of more extensive colon surgery. Gut 2011; 60 (7): 950-957

290 Stupart DA et al. Surgery for colonic cancer in HNPCC: total vs. segmental colectomy. Colorectal Dis 2011; 13 (12): 1395-1399

291 Schmeler KM et al. Prophylactic surgery to reduce the risk of gynecologic cancers in the Lynch syndrome. N Engl J Med 2006; 354 (3): 261-269

292 Church JM et al. Teenagers with familial adenomatous polyposis: what is their risk for colorectal cancer? Dis Colon Rectum 2002; 45 (7): $887-889$

293 King JE et al. Care of patients and their families with familial adenomatous polyposis. Mayo Clin Proc 2000; 75 (1): 57-67

294 Bertario $L$ et al. Causes of death and postsurgical survival in familial adenomatous polyposis: results from the Italian Registry. Italian Registry of Familial Polyposis Writing Committee. Semin Surg Oncol 1994; 10 (3): $225-234$

295 Galle TS, Juel K, Bulow S. Causes of death in familial adenomatous polyposis. Scand J Gastroenterol 1999; 34 (8): 808-812

296 Heiskanen I, Luostarinen T, Jarvinen HJ. Impact of screening examinations on survival in familial adenomatous polyposis. Scand J Gastroenterol 2000; 35 (12): 1284-1287

297 Vasen HF et al. Decision analysis in the surgical treatment of patients with familial adenomatous polyposis: a Dutch-Scandinavian collaborative study including 659 patients. Gut 2001; 49 (2): $231-235$

298 Bulow S. Results of national registration of familial adenomatous polyposis. Gut 2003; 52 (5): $742-746$

299 Ficari $\mathrm{F}$ et al. APC gene mutations and colorectal adenomatosis in $\mathrm{fa}-$ milial adenomatous polyposis. Br J Cancer 2000; 82 (2): 348 - 353

300 Bulow S. Clinical features in familial polyposis coli. Results of the Danish Polyposis Register. Dis Colon Rectum 1986; 29 (2): 102 - 107

301 De Cosse JJ et al. Rectal cancer risk in patients treated for familial adenomatous polyposis. The Leeds Castle Polyposis Group. Br J Surg 1992; 79 (12): $1372-1375$

302 Nyam DC et al. Ileal pouch-anal canal anastomosis for familial adenomatous polyposis: early and late results. Ann Surg 1997; 226 (4): 514-519; discussion 519-521

303 Parc YR et al. Familial adenomatous polyposis: results after ileal pouch-anal anastomosis in teenagers. Dis Colon Rectum 2000; 43 (7): 893-898; discussion 898-902

304 Van Duijvendijk P et al. Quality of life after total colectomy with ileorectal anastomosis or proctocolectomy and ileal pouch-anal anastomosis for familial adenomatous polyposis. Br J Surg 2000; 87 (5): 590-596

305 Church J et al. Risk of rectal cancer in patients after colectomy and ileorectal anastomosis for familial adenomatous polyposis: a function of available surgical options. Dis Colon Rectum 2003; 46 (9): 1175 1181 
306 Will OC et al. Familial adenomatous polyposis and the small bowel: a loco-regional review and current management strategies. Pathol Res Pract 2008; 204 (7): 449-458

307 Spigelman $A D$ et al. Upper gastrointestinal cancer in patients with familial adenomatous polyposis. Lancet 1989; 2 (8666): 783 - 785

308 Burke CA et al. The natural history of untreated duodenal and ampullary adenomas in patients with familial adenomatous polyposis followed in an endoscopic surveillance program. Gastrointest Endosc 1999; 49 (3): 358 - 364

309 Matsumoto T et al. Natural history of ampullary adenoma in familial adenomatous polyposis: reconfirmation of benign nature during extended surveillance. Am J Gastroenterol 2000; 95 (6): 1557-1562

310 Moozar KL et al. Slow progression of periampullary neoplasia in familial adenomatous polyposis. J Gastrointest Surg 2002; 6 (6): 831 837; discussion 837

311 Saurin JC et al. The influence of mutation site and age on the severity of duodenal polyposis in patients with familial adenomatous polyposis. Gastrointest Endosc 2002; 55 (3): 342 - 347

312 Saurin JC et al. Surveillance of duodenal adenomas in familial adenomatous polyposis reveals high cumulative risk of advanced disease. J Clin Oncol 2004; 22 (3): 493 - 498

313 Vasen HF et al. Decision analysis in the management of duodenal adenomatosis in familial adenomatous polyposis. Gut 1997; 40: 716 719

314 Bulow $S$ et al. Duodenal adenomatosis in familial adenomatous polyposis. Gut 2004; 53 (3): 381 - 386

315 Kadmon M, Tandara A, Herfarth C. Duodenal adenomatosis in familial adenomatous polyposis coli. A review of the literature and results from the Heidelberg Polyposis Register. Int J Colorectal Dis 2001; 16 (2): $63-75$

316 Norton ID et al. Safety and outcome of endoscopic snare excision of the major duodenal papilla. Gastrointest Endosc 2002; 56 (2): 239 243

317 Kalady MF et al. Pancreas-preserving duodenectomy in the management of duodenal familial adenomatous polyposis. J Gastrointest Surg 2002; 6 (1): $82-87$

318 Parc Y, Mabrut JY, Shields C. Surgical management of the duodenal manifestations of familial adenomatous polyposis. Br J Surg 2011; 98 (4): $480-484$

319 Vos tot Nederveen Cappel WH et al. Worldwide survey among polyposis registries of surgical management of severe duodenal adenomatosis in familial adenomatous polyposis. Br J Surg 2003; 90 (6): 705 710

320 Heiskanen I, Kellokumpu I, Jarvinen H. Management of duodenal adenomas in 98 patients with familial adenomatous polyposis. Endoscopy $1999 ; 31$ (6): $412-416$

321 Norton ID et al. Endoscopic surveillance and ablative therapy for periampullary adenomas. Am J Gastroenterol 2001; 96 (1): 101 - 106

322 Bleau BL, Gostout CJ. Endoscopic treatment of ampullary adenomas in familial adenomatous polyposis. J Clin Gastroenterol 1996; 22 (3): $237-241$

323 Soravia $C$ et al. Desmoid disease in patients with familial adenomatous polyposis. Dis Colon Rectum 2000; 43 (3): 363 - 369

324 Bertario L et al. Multiple approach to the exploration of genotypephenotype correlations in familial adenomatous polyposis. J Clin Oncol 2003; 21 (9): $1698-1707$

325 Friedl $W$ et al. Can APC mutation analysis contribute to therapeutic decisions in familial adenomatous polyposis? Experience from 680 FAP families. Gut 2001; 48 (4): 515-521

326 Janinis $J$ et al. The pharmacological treatment of aggressive fibromatosis: a systematic review. Ann Oncol 2003; 14 (2): 181 - 190

327 Tonelli $F$ et al. Treatment of desmoids and mesenteric fibromatosis in familial adenomatous polyposis with raloxifene. Tumori 2003; 89 (4): $391-396$

328 Gega $M$ et al. Successful chemotherapeutic modality of doxorubicin plus dacarbazine for the treatment of desmoid tumors in association with familial adenomatous polyposis. J Clin Oncol 2006; 24 (1): 102 105

329 Skapek SX et al. Vinblastine and methotrexate for desmoid fibromatosis in children: results of a Pediatric Oncology Group Phase II Trial. J Clin Oncol 2007; 25 (5): 501 - 506

330 Latchford AR et al. A 10-year review of surgery for desmoid disease associated with familial adenomatous polyposis. Br J Surg 2006; 93 (10): $1258-1264$
331 Möslein G. Current ideas in desmoid tumours: invited commentary. Fam Cancer 2006; 5: 287-288

332 Clark SK, Phillips RK. Desmoids in familial adenomatous polyposis. Br J Surg 1996; 83 (11): 1494-1504

333 Nieuwenhuis $\mathrm{MH}$ et al. Evaluation of management of desmoid tumours associated with familial adenomatous polyposis in Dutch patients. Br J Cancer 2011; 104 (1): 37 - 42

334 Huang $K$ et al. Prognostic factors for extra-abdominal and abdominal wall desmoids: a 20-year experience at a single institution. J Surg Oncol 2009; 100 (7): $563-569$

335 Salas $S$ et al. Prognostic factors influencing progression-free survival determined from a series of sporadic desmoid tumors: a wait-andsee policy according to tumor presentation. J Clin Oncol 2011; 29 (26): $3553-3558$

336 Bulow C, Bulow S. Is screening for thyroid carcinoma indicated in familial adenomatous polyposis? The Leeds Castle Polyposis Group. Int J Colorectal Dis 1997; 12 (4): 240-242

337 Jarrar AM et al. Screening for thyroid cancer in patients with familial adenomatous polyposis. Ann Surg 2011; 253 (3): 515 - 521

338 Martayan A et al. Gene variants associated to malignant thyroid disease in familial adenomatous polyposis: a novel APC germline mutation. J Endocrinol Invest 2010; 33 (9): 603 -606

339 Herraiz $M$ et al. Prevalence of thyroid cancer in familial adenomatous polyposis syndrome and the role of screening ultrasound examinations. Clin Gastroenterol Hepatol 2007; 5 (3): 367 - 373

340 Bell B, Mazzaferri EL. Familial adenomatous polyposis (Gardner's syndrome) and thyroid carcinoma. A case report and review of the literature. . Dig Dis Sci 1993; 38 (1): 185-190

341 Perrier ND et al. Thyroid cancer in patients with familial adenomatous polyposis. World J Surg 1998; 22 (7): 738 - 742; discussion 743

342 Hughes LJ, Michels VV. Risk of hepatoblastoma in familial adenomatous polyposis. Am J Med Genet 1992; 43 (6): 1023 - 1025

343 Thomas $D$ et al. Familial hepatoblastoma and APC gene mutations: renewed call for molecular research. Eur J Cancer 2003; 39 (15): 2200 2204

344 Aretz $S$ et al. Should children at risk for familial adenomatous polyposis be screened for hepatoblastoma and children with apparently sporadic hepatoblastoma be screened for APC germline mutations? Pediatr Blood Cancer 2006; 47 (6): 811 - 818

345 Vasen HF et al. Guidelines for the clinical management of familial adenomatous polyposis (FAP). Gut 2008; 57 (5): 704-713

346 Waddell WR, Loughry RW. Sulindac for polyposis of the colon. J Surg Oncol 1983; $24(1): 83-7$

347 Labayle $D$ et al. Sulindac causes regression of rectal polyps in familial adenomatous polyposis. Gastroenterology 1991; 101 (3): 635-639

348 Giardiello FM et al. Treatment of colonic and rectal adenomas with sulindac in familial adenomatous polyposis. N Engl J Med 1993; 328 (18): $1313-1316$

349 Cruz-Correa $M$ et al. Long-term treatment with sulindac in familial adenomatous polyposis: a prospective cohort study. Gastroenterology $2002 ; 122$ (3): 641 - 645

350 Winde $\mathrm{G}$ et al. Complete reversion and prevention of rectal adenomas in colectomized patients with familial adenomatous polyposis by rectal low-dose sulindac maintenance treatment. Advantages of low-dose nonsteroidal anti-inflammatory drug regimen in reversing adenomas exceeding 33 months. Dis Colon Rectum 1995; 38 (8): 813-830

351 Giardiello FM et al. Primary chemoprevention of familial adenomatous polyposis with sulindac. N Engl J Med 2002; 346 (14): 1054 1059

352 Steinbach $G$ et al. The effect of celecoxib, a cyclooxygenase-2 inhibitor, in familial adenomatous polyposis. N Engl J Med 2000; 342 (26): $1946-1952$

353 Phillips RK et al. A randomised, double blind, placebo controlled study of celecoxib, a selective cyclooxygenase 2 inhibitor, on duodenal polyposis in familial adenomatous polyposis. Gut 2002; 50 (6): 857 860

354 Solomon SD et al. Cardiovascular risk of celecoxib in 6 randomized placebo-controlled trials: the cross trial safety analysis. Circulation 2008; 117 (16): $2104-2113$

355 Matsuo S et al. Attenuated familial adenomatous polyposis associated with advanced rectal cancer in a 16-year-old boy: report of a case. Surg Today 2001; 31 (11): 1020-1023

356 Leggett BA et al. Severe upper gastrointestinal polyposis associated with sparse colonic polyposis in a familial adenomatous polyposis 
family with an APC mutation at codon 1520. Gut 1997; 41 (4): 518 521

357 Zwick A et al. Gastric adenocarcinoma and dysplasia in fundic gland polyps of a patient with attenuated adenomatous polyposis coli. Gastroenterology 1997; 113 (2): 659-663

358 Hofgartner WT et al. Gastric adenocarcinoma associated with fundic gland polyps in a patient with attenuated familial adenomatous polyposis. Am J Gastroenterol 1999; 94 (8): 2275-2281

359 Jones $\mathrm{N}$ et al. Increased colorectal cancer incidence in obligate carriers of heterozygous mutations in MUTYH. Gastroenterology 2009; 137 (2): 489-494, 494 e1; quiz 725-726

360 Farrington SM et al. Germline susceptibility to colorectal cancer due to base-excision repair gene defects. Am J Hum Genet 2005; 77 (1): $112-119$

361 Nielsen $M$ et al. Analysis of MUTYH genotypes and colorectal phenotypes in patients With MUTYH-associated polyposis. Gastroenterology 2009; 136 (2): $471-476$

362 Nascimbeni $R$ et al. Rectum-sparing surgery may be appropriate for biallelic MutYH-associated polyposis. Dis Colon Rectum 2010; 53 (12): $1670-1675$

363 Nielsen $M$ et al. Duodenal carcinoma in MUTYH-associated polyposis. J Clin Pathol 2006; 59 (11): $1212-1215$

364 Zbuk KM, Eng C. Hamartomatous polyposis syndromes. Nat Clin Pract Gastroenterol Hepatol 2007; 4 (9): 492-502

365 Aretz $S$ et al. High proportion of large genomic STK11 deletions in Peutz-Jeghers syndrome. Hum Mutat 2005; 26 (6): 513-519

366 Hinds $R$ et al. Complications of childhood Peutz-Jeghers syndrome: implications for pediatric screening. J Pediatr Gastroenterol Nutr 2004; 39 (2): 219-220

367 Reid JD. Intestinal carcinoma in the Peutz-Jeghers syndrome. Jama 1974; 229 (7): $833-834$

368 Burdick D, Prior JT. Peutz-Jeghers syndrome. A clinicopathologic study of a large family with a 27-year follow-up. Cancer 1982; 50 (10): $2139-2146$

369 Giardiello FM et al. Increased risk of cancer in the Peutz-Jeghers syndrome. N Engl J Med 1987; 316 (24): 1511 - 1514

370 Foley TR, McGarrity TJ, Abt AB. Peutz-Jeghers syndrome: a clinicopathologic survey of the "Harrisburg family" with a 49-year follow-up. Gastroenterology 1988; 95 (6): 1535-1540

371 Spigelman AD, Murday V, Phillips RK. Cancer and the Peutz-Jeghers syndrome. Gut 1989; 30 (11): $1588-1590$

372 Hizawa $K$ et al. Neoplastic transformation arising in Peutz-Jeghers polyposis. Dis Colon Rectum 1993; 36: 953-957

373 Boardman LA et al. Increased risk for cancer in patients with the Peutz-Jeghers syndrome. Ann Intern Med 1998; 128 (11): 896-899

374 Mehenni $\mathrm{H}$ et al. Cancer risks in LKB1 germline mutation carriers. Gut 2006; 55 (7): $984-990$

375 Giardiello FM et al. Very high risk of cancer in familial Peutz-Jeghers syndrome. Gastroenterology 2000; 119 (6): 1447 - 1453

376 Hearle $N$ et al. Frequency and spectrum of cancers in the Peutz-Jeghers syndrome. Clin Cancer Res 2006; 12 (10): 3209-3215

377 Sweet $K$ et al. Molecular classification of patients with unexplained hamartomatous and hyperplastic polyposis. JAMA 2005; 294 (19): $2465-2473$

378 Aretz $S$ et al. High proportion of large genomic deletions and a genotype phenotype update in 80 unrelated families with juvenile polyposis syndrome. J Med Genet 2007; 44 (11): 702 - 709

379 Gallione $C J$ et al. A combined syndrome of juvenile polyposis and hereditary haemorrhagic telangiectasia associated with mutations in MADH4 (SMAD4). Lancet 2004; 363 (9412): 852 - 859

380 Watanabe A et al. Familial juvenile polyposis of the stomach. Gastroenterology 1979; 77 (1): 148-151

381 Jass JR et al. Juvenile polyposis - a precancerous condition. Histopathology 1988; 13 (6): 619-630

382 Sassatelli $R$ et al. Generalized juvenile polyposis with mixed pattern and gastric cancer. Gastroenterology 1993; 104 (3): 910-915

383 Coburn MC et al. Malignant potential in intestinal juvenile polyposis syndromes. Ann Surg Oncol 1995; 2 (5): 386-391

384 Desai DC et al. Juvenile polyposis. Br J Surg 1995; 82 (1): 14-17

385 Delnatte $C$ et al. Contiguous gene deletion within chromosome arm $10 q$ is associated with juvenile polyposis of infancy, reflecting cooperation between the BMPR1A and PTEN tumor-suppressor genes. Am J Hum Genet 2006; 78 (6): 1066 - 1074

386 Hobert JA, Eng C. PTEN hamartoma tumor syndrome: an overview. Genet Med 2009; 11 (10): 687-694
387 Tan $\mathrm{MH}$ et al. Lifetime cancer risks in individuals with germline PTEN mutations. Clin Cancer Res 2012; 18 (2): 400 - 407

388 Orloff MS, Eng C. Genetic and phenotypic heterogeneity in the PTEN hamartoma tumour syndrome. Oncogene 2008; 27 (41): 5387-5397

389 Marsh DJ et al. PTEN mutation spectrum and genotype-phenotype correlations in Bannayan-Riley-Ruvalcaba syndrome suggest a single entity with Cowden syndrome. Hum Mol Genet 1999; 8 (8): 1461 1472

390 Jeevaratnam $P$ et al. Familial giant hyperplastic polyposis predisposing to colorectal cancer: a new hereditary bowel cancer syndrome. J Pathol 1996; 179 (1): $20-25$

391 Leggett BA et al. Hyperplastic polyposis: association with colorectal cancer. Am J Surg Pathol 2001; 25 (2): 177-184

392 Renaut AJ, Douglas PR, Newstead GL. Hyperplastic polyposis of the colon and rectum. Colorectal Dis 2002; 4 (3): 213-215

393 Jaeger EE et al. An ancestral Ashkenazi haplotype at the HMPS/CRAC1 locus on 15q13-q14 is associated with hereditary mixed polyposis syndrome. Am J Hum Genet 2003; 72 (5): 1261 - 1267

394 Rozen P, Samuel Z, Brazowski E. A prospective study of the clinical, genetic, screening, and pathologic features of a family with hereditary mixed polyposis syndrome. Am J Gastroenterol 2003; 98 (10): $2317-2320$

395 Beggs $A D$ et al. Peutz-Jeghers syndrome: a systematic review and recommendations for management. Gut 2010; 59 (7): 975 -986

396 Dignass A et al. Updated German guideline on diagnosis and treatment of ulcerative colitis, 2011. Z Gastroenterol 2011; 49 (9): 1276 1341

397 Collins PD et al. Strategies for detecting colon cancer and/or dysplasia in patients with inflammatory bowel disease. Cochrane Database Syst Rev 2006; 2: CD000279

398 Rutter MD et al. Thirty-year analysis of a colonoscopic surveillance program for neoplasia in ulcerative colitis. Gastroenterology 2006 130 (4): $1030-1038$

399 Lutgens MW et al. High frequency of early colorectal cancer in inflammatory bowel disease. Gut 2008; 57 (9): 1246 - 1251

$400 \mathrm{Lim} \mathrm{CH}$ et al. Ten year follow up of ulcerative colitis patients with and without low grade dysplasia. Gut 2003; 52 (8): 1127-1132

401 Connell WR et al. Factors affecting the outcome of endoscopic surveillance for cancer in ulcerative colitis. Gastroenterology 1994; 107 (4): 934-944

402 Claessen MM et al. More right-sided IBD-associated colorectal cancer in patients with primary sclerosing cholangitis. Inflamm Bowel Dis 2009; 15 (9): $1331-1336$

403 Soetikno RM et al. Increased risk of colorectal neoplasia in patients with primary sclerosing cholangitis and ulcerative colitis: a metaanalysis. Gastrointest Endosc 2002; 56 (1): 48 - 54

404 Scarpa $M$ et al. Systematic review of dysplasia after restorative proctocolectomy for ulcerative colitis. Br J Surg 2007; 94 (5): 534-545

405 Hoffmann JC et al. Clinical practice guideline on diagnosis and treatment of Crohn's disease. Z Gastroenterol 2008; 46 (9): 1094 - 1146

406 Rex DK et al. Quality in the technical performance of colonoscopy and the continuous quality improvement process for colonoscopy: recommendations of the U.S. Multi-Society Task Force on Colorectal Cancer. Am J Gastroenterol 2002; 97 (6): 1296-1308

407 Barclay RL et al. Colonoscopic withdrawal times and adenoma detection during screening colonoscopy. N Engl J Med 2006; 355 (24): $2533-2541$

408 Simmons DT et al. Impact of endoscopist withdrawal speed on polyp yield: implications for optimal colonoscopy withdrawal time. Aliment Pharmacol Ther 2006; 24 (6): 965-971

409 Lieberman $D$. A call to action - measuring the quality of colonoscopy. N Engl J Med 2006; 355 (24): 2588 - 2589

410 Pickhardt PJ et al. Computed tomographic virtual colonoscopy to screen for colorectal neoplasia in asymptomatic adults. N Engl J Med 2003; 349 (23): $2191-2200$

411 Robertson DJ et al. Colorectal cancer in patients under close colonoscopic surveillance. Gastroenterology 2005; 129 (1): 34-41

412 Hosokawa 0 et al. Invasive colorectal cancer detected up to 3 years after a colonoscopy negative for cancer. Endoscopy 2003; 35 (6): 506-510

413 Bressler B et al. Colonoscopic miss rates for right-sided colon cancer: a population-based analysis. Gastroenterology 2004; 127 (2): 452 456

414 Leaper $M$ et al. Reasons for failure to diagnose colorectal carcinoma at colonoscopy. Endoscopy 2004; 36 (6): 499-503 
415 Pabby A et al. Analysis of colorectal cancer occurrence during surveillance colonoscopy in the dietary Polyp Prevention Trial. Gastrointest Endosc 2005; 61 (3): 385 - 391

416 Cotton PB et al. Computed tomographic colonography (virtual colonoscopy): a multicenter comparison with standard colonoscopy for detection of colorectal neoplasia. Jama 2004; 291 (14): 1713-1719

417 Rockey DC et al. Analysis of air contrast barium enema, computed tomographic colonography, and colonoscopy: prospective comparison. Lancet 2005; 365 (9456): 305 - 311

418 Halligan $S$ et al. CT colonography in the detection of colorectal polyps and cancer: systematic review, meta-analysis, and proposed minimum data set for study level reporting. Radiology 2005; 237 (3): $893-904$

419 Mulhall BP, Veerappan GR, Jackson JL. Meta-analysis: computed tomographic colonography. Ann Intern Med 2005; 142 (8): 635 - 650

420 Johnson CD et al. Comparison of the relative sensitivity of CT colonography and double-contrast barium enema for screen detection of colorectal polyps. Clin Gastroenterol Hepatol 2004; 2 (4): 314 - 321

421 Purkayastha $S$ et al. Magnetic resonance colonography versus colonoscopy as a diagnostic investigation for colorectal cancer: a metaanalysis. Clin Radiol 2005; 60 (9): 980 - 989

422 Hartmann $D$ et al. Colorectal polyps: detection with dark-lumen MR colonography versus conventional colonoscopy. Radiology 2006; 238 (1): $143-149$

423 Florie J et al. MR colonography with limited bowel preparation compared with optical colonoscopy in patients at increased risk for colorectal cancer. Radiology 2007; 243 (1): 122 -131

424 Morrin MM et al. Virtual colonoscopy: a kinder, gentler colorectal cancer screening test? Lancet 1999; 354 (9184): 1048-1049

425 Morrin MM et al. Role of virtual computed tomographic colonography in patients with colorectal cancers and obstructing colorectal lesions. Dis Colon Rectum 2000; 43 (3): 303 - 311

426 Neri $E$ et al. Colorectal cancer: role of CT colonography in preoperative evaluation after incomplete colonoscopy. Radiology 2002; 223 (3): 615-619

427 Hartmann D et al. Incomplete conventional colonoscopy: magnetic resonance colonography in the evaluation of the proximal colon. Endoscopy 2005; 37 (9): 816-820

428 Arora A, Singh P. Colonoscopy in patients 80 years of age and older is safe, with high success rate and diagnostic yield. Gastrointest Endosc 2004; 60 (3): $408-413$

429 Karajeh MA, Sanders DS, Hurlstone DP. Colonoscopy in elderly people is a safe procedure with a high diagnostic yield: a prospective comparative study of 2000 patients. Endoscopy 2006; 38 (3): 226-230

430 Kiesslich $R$ et al. Methylene blue-aided chromoendoscopy for the detection of intraepithelial neoplasia and colon cancer in ulcerative colitis. Gastroenterology 2003; 124 (4): 880-888

431 Hurlstone DP et al. The role of high-magnification-chromoscopic colonoscopy in hereditary nonpolyposis colorectal cancer screening: a prospective "back-to-back" endoscopic study. Am J Gastroenterol 2005; 100 (10): $2167-2173$

432 Hurlstone DP et al. Indigo carmine-assisted high-magnification chromoscopic colonoscopy for the detection and characterisation of intraepithelial neoplasia in ulcerative colitis: a prospective evaluation. Endoscopy 2005; 37 (12): 1186-1192

433 Lee JH et al. Detection of colorectal adenomas by routine chromoendoscopy with indigocarmine. Am J Gastroenterol 2003; 98 (6): $1284-1288$

434 Hurlstone DP et al. Detecting diminutive colorectal lesions at colonoscopy: a randomised controlled trial of pan-colonic versus targeted chromoscopy. Gut 2004; 53 (3): $376-380$

435 Trecca A et al. Advanced diagnostic tools: virtual colonoscopy and magnifying chromoendoscopy. Tech Coloproctol 2004; 8 (Suppl 2): s279-s282

436 Lapalus MG et al. Does chromoendoscopy with structure enhancement improve the colonoscopic adenoma detection rate? Endoscopy 2006; 38 (5): $444-448$

437 Kudo S et al. Colonoscopic diagnosis and management of nonpolypoid early colorectal cancer. World J Surg 2000; 24 (9): 1081 - 1090

438 Kiesslich $R$ et al. Chromoendoscopy with indigocarmine improves the detection of adenomatous and nonadenomatous lesions in the colon. Endoscopy 2001; 33 (12): 1001 - 1006

439 Kudo $S$ et al. Pit pattern in colorectal neoplasia: endoscopic magnifying view. Endoscopy 2001; 33 (4): 367 - 373
440 Brooker JC et al. Total colonic dye-spray increases the detection of diminutive adenomas during routine colonoscopy: a randomized controlled trial. Gastrointest Endosc 2002; 56 (3): 333 - 338

441 Eisen GM et al. High-resolution chromoendoscopy for classifying colonic polyps: a multicenter study. Gastrointest Endosc 2002; 55 (6): $687-694$

442 Tsuda $S$ et al. Flat and depressed colorectal tumours in a southern Swedish population: a prospective chromoendoscopic and histopathological study. Gut 2002; 51 (4): 550-555

443 Bianco MA et al. Predictive value of magnification chromoendoscopy for diagnosing invasive neoplasia in nonpolypoid colorectal lesions and stratifying patients for endoscopic resection or surgery. Endoscopy 2006; 38 (5): $470-476$

444 Kato $S$ et al. Magnifying colonoscopy as a non-biopsy technique for differential diagnosis of non-neoplastic and neoplastic lesions. World J Gastroenterol 2006; 12 (9): 1416-1420

445 Hurlstone DP et al. Endoscopic morphological anticipation of submucosal invasion in flat and depressed colorectal lesions: clinical implications and subtype analysis of the kudo type $\mathrm{V}$ pit pattern using high-magnification-chromoscopic colonoscopy. Colorectal Dis 2004; $6(5): 369-375$

446 Hurlstone DP et al. Efficacy of high magnification chromoscopic colonoscopy for the diagnosis of neoplasia in flat and depressed lesions of the colorectum: a prospective analysis. Gut 2004; 53 (2): 284-290

447 Chiu HM et al. A prospective comparative study of narrow-band imaging, chromoendoscopy, and conventional colonoscopy in the diagnosis of colorectal neoplasia. Gut 2007; 56 (3): 373 - 379

448 Dekker $E$ et al. Narrow-band imaging compared with conventional colonoscopy for the detection of dysplasia in patients with longstanding ulcerative colitis. Endoscopy 2007; 39 (3): 216-221

449 Su MY et al. Comparative study of conventional colonoscopy, chromoendoscopy, and narrow-band imaging systems in differential diagnosis of neoplastic and nonneoplastic colonic polyps. Am J Gastroenterol 2006; 101 (12): $2711-2716$

450 Kiesslich $R$ et al. Confocal laser endoscopy for diagnosing intraepithelial neoplasias and colorectal cancer in vivo. Gastroenterology 2004; 127 (3): $706-713$

451 Nivatvongs $S$. Complications in colonoscopic polypectomy. An experience with 1,555 polypectomies. Dis Colon Rectum 1986; 29 (12): $825-830$

452 Reiertsen 0 et al. Complications of fiberoptic gastrointestinal endoscopy - five years' experience in a central hospital. Endoscopy 1987 $19(1): 1-6$

453 Waye JD, Lewis BS, Yessayan S. Colonoscopy: a prospective report of complications. J Clin Gastroenterol 1992; 15 (4): 347 - 351

454 Waye JD, Kahn O, Auerbach ME. Complications of colonoscopy and flexible sigmoidoscopy. Gastrointest Endosc Clin N Am 1996; 6 (2): $343-377$

455 Hsieh $\mathrm{YH}$ et al. Is submucosal epinephrine injection necessary before polypectomy? A prospective, comparative study. Hepatogastroenterology 2001; 48 (41): $1379-1382$

456 Karita M, Cantero D, Okita K. Endoscopic diagnosis and resection treatment for flat adenoma with severe dysplasia. Am J Gastroenterol 1993; 88 (9): 1421 - 1423

457 Kudo S et al. Endoscopic diagnosis and treatment of early colorectal cancer. World J Surg 1997; 21 (7): $694-701$

458 Heldwein W et al. The Munich Polypectomy Study (MUPS): prospective analysis of complications and risk factors in 4000 colonic snare polypectomies. Endoscopy 2005; 37 (11): 1116-1122

459 Di Giorgio P et al. Detachable snare versus epinephrine injection in the prevention of postpolypectomy bleeding: a randomized and controlled study. Endoscopy 2004; 36 (10): 860-863

460 Dobrowolski $S$ et al. Prophylactic submucosal saline-adrenaline injection in colonoscopic polypectomy: prospective randomized study. Surg Endosc 2004; 18 (6): 990 - 993

461 Katsinelos $P$ et al. Endoloop-assisted polypectomy for large pedunculated colorectal polyps. Surg Endosc 2006; 20 (8): 1257-1261

462 Paspatis GA et al. A prospective, randomized comparison of adrenaline injection in combination with detachable snare versus adrenaline injection alone in the prevention of postpolypectomy bleeding in large colonic polyps. Am J Gastroenterol 2006; 101 (12): 2805; quiz 2913

463 Shioji K et al. Prophylactic clip application does not decrease delayed bleeding after colonoscopic polypectomy. Gastrointest Endosc 2003; 57 (6): $691-694$ 
464 Shiffman ML, Farrel MT, Yee YS. Risk of bleeding after endoscopic biopsy or polypectomy in patients taking aspirin or other NSAIDS. Gastrointest Endosc 1994; 40 (4): 458 -462

465 Hui AJ et al. Risk of colonoscopic polypectomy bleeding with anticoagulants and antiplatelet agents: analysis of 1657 cases. Gastrointest Endosc 2004; 59 (1): 44- 48

466 Yousfi $M$ et al. Postpolypectomy lower gastrointestinal bleeding: potential role of aspirin. Am J Gastroenterol 2004; 99 (9): 1785 - 1789

467 Ernst $A$ et al. Effect of routine clopidogrel use on bleeding complications after transbronchial biopsy in humans. Chest 2006; 129 (3): $734-737$

468 Hurlstone DP et al. A prospective analysis of extended endoscopic mucosal resection for large rectal villous adenomas: an alternative technique to transanal endoscopic microsurgery. Colorectal Dis 2005; 7 (4): 339-344

469 Gondal $G$ et al. Biopsy of colorectal polyps is not adequate for grading of neoplasia. Endoscopy 2005; 37 (12): 1193-1197

470 Waye JD et al. Small colon polyps. Am J Gastroenterol 1988; 83 (2): $120-122$

471 O'Brien MJ et al. The National Polyp Study. Patient and polyp characteristics associated with high-grade dysplasia in colorectal adenomas. Gastroenterology 1990; 98 (2): 371 - 379

472 Nusko $G$ et al. Risk of invasive carcinoma in colorectal adenomas assessed by size and site. Int J Colorectal Dis 1997; 12 (5): 267-271

473 Bond JH. Clinical relevance of the small colorectal polyp. Endoscopy 2001; 33 (5): $454-457$

474 Arumugam PJ et al. Severe dysplastic lesions in the colon - how aggressive should we be? Colorectal Dis 2002; 4 (5): 345-347

475 Gschwantler $M$ et al. High-grade dysplasia and invasive carcinoma in colorectal adenomas: a multivariate analysis of the impact of adenoma and patient characteristics. Eur J Gastroenterol Hepatol 2002; 14 (2): $183-188$

476 Burton S et al. Reliability of pre-operative biopsies in the histological grading of colorectal adenocarcinomas. Ann R Coll Surg Engl 2003; 85 (1): $23-25$

477 Hamilton SR, Aaltonen LA. Pathology and Genetics of Tumours of the Digestive System. World Health Organization Classification of Tumours. ed. Lyon: IARC Press; 2000

478 Jass JR. Classification of colorectal cancer based on correlation of clinical, morphological and molecular features. Histopathology 2007; 50 (1): $113-130$

479 Makinen MJ. Colorectal serrated adenocarcinoma. Histopathology 2007; 50 (1): 131 - 150

480 Deinlein $P$ et al. Risk factors for lymphatic metastasis from pT1 colorectal adenocarcinoma. Pathologe 2003; 24 (5): 387-393

481 Hermanek P. Polypectomy in the colorectum: histological and oncological aspects. Endoscopy 1983; 15: 158-161

482 Kikuchi $R$ et al. Management of early invasive colorectal cancer. Risk of recurrence and clinical guidelines. Dis Colon Rectum 1995; 38 (12): $1286-1295$

483 Okuyama T, Oya M, Ishikawa $H$. Budding as a risk factor for lymph node metastasis in pT1 or pT2 well-differentiated colorectal adenocarcinoma. Dis Colon Rectum 2002; 45 (5): 628 - 634

484 Fruhmorgen $P$ et al. Endoscopic Therapy of Early Colorectal Cancer (pT1) - A Prospective Study. Z Gastroenterol 2003; 41 (8): 703 - 710

485 Frühmorgen P et al. Kolorektale Polypen. In: Empfehlungen der Deutschen Gesellschaft für Verdauungs- und Stoffwechselkrankheiten (DGVS) für die Durchführung endoskopischer Untersuchungen 2000: $127-134$

486 Okabe S et al. Lymph node metastasis in T1 adenocarcinoma of the colon and rectum. J Gastrointest Surg 2004; 8 (8): 1032 - 1039; discussion 1039-1040

487 Yamamoto $S$ et al. The risk of lymph node metastasis in T1 colorectal carcinoma. Hepatogastroenterology 2004; 51 (58): 998 - 1000

488 Hassan C et al. Histologic risk factors and clinical outcome in colorectal malignant polyp: a pooled-data analysis. Dis Colon Rectum 2005; 48 (8): $1588-1596$

489 Ueno $\mathrm{H}$ et al. Risk factors for an adverse outcome in early invasive colorectal carcinoma. Gastroenterology 2004; 127 (2): 385-394

490 The Paris endoscopic classification of superficial neoplastic lesions: esophagus, stomach, and colon: November 30 to December 12002. Gastrointest Endosc 2003; 58 (Suppl 6): S3 - S43

491 Nascimbeni $R$ et al. Risk of lymph node metastasis in T1 carcinoma of the colon and rectum. Dis Colon Rectum 2002; 45 (2): 200-206
492 Seitz $U$ et al. Is endoscopic polypectomy an adequate therapy for malignant colorectal adenomas? Presentation of 114 patients and review of the literature. Dis Colon Rectum 2004; 47 (11): 17891796DOI: ; discussion 1796-1797

493 Provenzale $D$ et al. Risk for colon adenomas in patients with rectosigmoid hyperplastic polyps. Ann Intern Med 1990; 113: 760 - 763

494 Rex DK et al. Distal colonic hyperplastic polyps do not predict proximal adenomas in asymptomatic average-risk subjects. Gastroenterology 1992; 102: $317-319$

495 Sciallero $S$ et al. Distal hyperplastic polyps do not predict proximal adenomas: results from a multicentric study of colorectal adenomas. Gastrointest Endosc 1997; 46 (2): 124-130

496 Koide $N$ et al. A case of hyperplastic polyposis of the colon with adenocarcinomas in hyperplastic polyps after long-term follow-up. Endoscopy 2002; 34 (6): 499-502

497 Bressler B et al. Rates of new or missed colorectal cancers after colonoscopy and their risk factors: a population-based analysis. Gastroenterology 2007; 132 (1): 96-102

498 Sawhney MS et al. Microsatellite instability in interval colon cancers. Gastroenterology 2006; 131 (6): 1700-1705

499 Winawer SJ et al. Guidelines for colonoscopy surveillance after polypectomy: a consensus update by the US Multi-Society Task Force on Colorectal Cancer and the American Cancer Society. Gastroenterology 2006; 130 (6): $1872-1885$

500 Higaki S et al. Long-term follow-up of large flat colorectal tumors resected endoscopically. Endoscopy 2003; 35 (10): 845 -849

501 Regula $\mathrm{J}$ et al. Argon plasma coagulation after piecemeal polypectomy of sessile colorectal adenomas: long-term follow-up study. Endoscopy 2003; 35 (3): $212-218$

502 Seitz $U$ et al. Long-term results of endoscopic removal of large colorectal adenomas. Endoscopy 2003; 35 (8): S41 - S44

503 Boix J et al. Endoscopic removal of large sessile colorectal adenomas: is it safe and effective? Dig Dis Sci 2007; 52 (3): 840-844

504 Baron JA et al. A randomized trial of aspirin to prevent colorectal adenomas. N Engl J Med 2003; 348 (10): 891 - 899

505 Benamouzig $R$ et al. Daily soluble aspirin and prevention of colorectal adenoma recurrence: one-year results of the APACC trial. Gastroenterology 2003; 125 (2): 328-336

506 Imperiale TF. Aspirin and the prevention of colorectal cancer. N Engl J Med 2003; 348 (10): 879-880

507 Bresalier RS et al. Cardiovascular events associated with rofecoxib in a colorectal adenoma chemoprevention trial. N Engl J Med 2005; 352 (11): $1092-1102$

508 Solomon SD et al. Cardiovascular risk associated with celecoxib in a clinical trial for colorectal adenoma prevention. N Engl J Med 2005; 352 (11): $1071-1080$

509 Psaty BM, Potter JD. Risks and benefits of celecoxib to prevent recurrent adenomas. N Engl J Med 2006; 355 (9): 950-952

510 Weingarten MA, Zalmanovici A, Yaphe J. Dietary calcium supplementation for preventing colorectal cancer and adenomatous polyps. Cochrane Database Syst Rev 2005; 3: CD003548

511 Fielding LP et al. Clinicopathological staging for colorectal cancer: an International Documentation System (IDS) and an International Comprehensive Anatomical Terminology (ICAT). J Gastroenterol Hepatol 1991; 6 (4): 325 - 344

512 Soreide 0 et al. International standardization and documentation of the treatment of rectal cancer. In: Soreide O, Norstein J, (eds) Rectal cancer surgery. Optimisation - standardization - documentation Berlin Heidelberg New York.: Springer; 1997: 405-445

513 UICC. TNM Classification of Malignant Tumours. Sobin LH, Wittekind C (ed.) 6th ed. New York: John Wiley \& Sons; 2002

514 Adjuvant therapy for patients with colon and rectal cancer. Jama 1990; 264 (11): $1444-1450$

515 Nelson $\mathrm{H}$ et al. Guidelines 2000 for colon and rectal cancer surgery. J Natl Cancer Inst 2001; 93 (8): 583-596

516 Pilipshen SJ et al. Patterns of pelvic recurrence following definitive resections of rectal cancer. Cancer 1984; 53 (6): 1354-1362

517 Burton $S$ et al. MRI directed multidisciplinary team preoperative treatment strategy: the way to eliminate positive circumferential margins? Br J Cancer 2006; 94 (3): 351 - 357

518 Segelman $J$ et al. Differences in multidisciplinary team assessment and treatment between patients with stage IV colon and rectal cancer. Colorectal Dis 2009; 11 (7): $768-774$

519 MacDermid $E$ et al. Improving patient survival with the colorectal cancer multi-disciplinary team. Colorectal Dis 2009; 11 (3): 291 - 295 
520 Barillari Pet al. Effect of preoperative colonoscopy on the incidence of synchronous and metachronous neoplasms. Acta Chir Scand 1990; 156 (2): 163 - 166

521 Faivre J. Endoscopic monitoring after excision of colorectal cancer. J Chir (Paris) 1997; 134 (7): $283-285$

522 Chen HS, Sheen-Chen SM. Synchronous and "early" metachronous colorectal adenocarcinoma: analysis of prognosis and current trends. Dis Colon Rectum 2000; 43 (8): 1093 - 1099

523 Floriani I et al. Performance of imaging modalities in diagnosis of liver metastases from colorectal cancer: a systematic review and metaanalysis. J Magn Reson Imaging 2010; 31 (1): 19-31

524 Rafaelsen SR, Jakobsen A. Contrast-enhanced ultrasound vs. multidetector-computed tomography for detecting liver metastases in colorectal cancer: a prospective, blinded, patient-by-patient analysis. Colorectal Dis 2011; 13 (4): 420-425

525 Quaia E et al. Comparison of contrast-enhanced ultrasonography versus baseline ultrasound and contrast-enhanced computed tomography in metastatic disease of the liver: diagnostic performance and confidence. Eur Radiol 2006; 16 (7): 1599 - 1609

526 Niekel MC, Bipat S, Stoker J. Diagnostic imaging of colorectal liver metastases with CT, MR imaging, FDG PET, and/or FDG PET/CT: a metaanalysis of prospective studies including patients who have not previously undergone treatment. Radiology 2010; 257 (3): 674-684

527 Mainenti PP et al. Detection of colo-rectal liver metastases: prospective comparison of contrast enhanced US, multidetector CT, PET/CT, and 1.5 Tesla MR with extracellular and reticulo-endothelial cell specific contrast agents. Abdom Imaging 2010; 35 (5): 511 - 521

528 McAndrew MR, Saba AK. Efficacy of routine preoperative computed tomography scans in colon cancer. Am Surg 1999; 65 (3): 205 - 208

529 Barton JB et al. The utility of routine preoperative computed tomography scanning in the management of veterans with colon cancer. Am J Surg 2002; 183 (5): 499-503

530 Dighe S et al. Diagnostic precision of CT in local staging of colon cancers: a meta-analysis. Clin Radiol 2010; 65 (9): 708 - 719

531 Abdel-Nabi $\mathrm{H}$ et al. Staging of primary colorectal carcinomas with fluorine-18 fluorodeoxyglucose whole-body PET: correlation with histopathologic and CT findings. Radiology 1998; 206 (3): $755-760$

532 Akiyoshi $T$ et al. Comparison of preoperative whole-body positron emission tomography with MDCT in patients with primary colorectal cancer. Colorectal Dis 2009; 11 (5): 464-469

533 Furukawa $\mathrm{H}$ et al. Positron emission tomography scanning is not superior to whole body multidetector helical computed tomography in the preoperative staging of colorectal cancer. Gut 2006; 55 (7): $1007-1011$

$534 \mathrm{Kam} \mathrm{MH}$ et al. Comparison of magnetic resonance imaging-fluorodeoxy-glucose positron emission tomography fusion with pathological staging in rectal cancer. Br J Surg 2010; 97 (2): 266-268

535 Kinner $S$ et al. Whole-body PET/CT-colonography: a possible new concept for colorectal cancer staging. Abdom Imaging 2007; 32 (5): 606-612

536 Llamas-Elvira JM et al. Fluorine-18 fluorodeoxyglucose PET in the preoperative staging of colorectal cancer. Eur J Nucl Med Mol Imaging 2007; 34 (6): $859-867$

537 Ono $\mathrm{K}$ et al. Comparison of diffusion-weighted MRI and 2-[fluorine18]-fluoro-2-deoxy-D-glucose positron emission tomography (FDGPET) for detecting primary colorectal cancer and regional lymph node metastases. J Magn Reson Imaging 2009; 29 (2): 336 - 340

538 Tateishi $U$ et al. Non-enhanced CT versus contrast-enhanced CT in integrated PET/CT studies for nodal staging of rectal cancer. Eur J Nucl Med Mol Imaging 2007; 34 (10): 1627-1634

539 Veit-Haibach $P$ et al. Diagnostic accuracy of colorectal cancer staging with whole-body PET/CT colonography. JAMA 2006; 296 (21): 2590 2600

540 Bassi MC et al. FDG-PET/CT imaging for staging and target volume delineation in preoperative conformal radiotherapy of rectal cancer. Int J Radiat Oncol Biol Phys 2008; 70 (5): 1423-1426

541 Davey K et al. The impact of 18-fluorodeoxyglucose positron emission tomography-computed tomography on the staging and management of primary rectal cancer. Dis Colon Rectum 2008; 51 (7): 997 1003

542 Gearhart SL et al. Improved staging with pretreatment positron emission tomography/computed tomography in low rectal cancer. Ann Surg Oncol 2006; 13 (3): $397-404$
543 Kantorova I et al. Routine (18)F-FDG PET preoperative staging of colorectal cancer: comparison with conventional staging and its impact on treatment decision making. J Nucl Med 2003; 44 (11): 1784-1788

544 Nagata K et al. PET/CT colonography for the preoperative evaluation of the colon proximal to the obstructive colorectal cancer. Dis Colon Rectum 2008; 51 (6): $882-890$

545 Park IJ et al. Efficacy of PET/CT in the accurate evaluation of primary colorectal carcinoma. Eur J Surg Oncol 2006; 32 (9): 941 - 947

546 Paskeviciute B et al. Impact of (18)F-FDG-PET/CT on staging and irradiation of patients with locally advanced rectal cancer. Strahlenther Onkol 2009; 185 (4): 260 - 265

547 Ruers TJ et al. Improved selection of patients for hepatic surgery of colorectal liver metastases with (18)F-FDG PET: a randomized study. J Nucl Med 2009; 50 (7): 1036-1041

548 Moulton CA. An Ontario Clinical Oncology Group (OCOG) randomized controlled trial (RCT) assessing FDG PET/CT in resectable liver colorectal adenocarcinoma metastases (CAM). J Clin Oncol 2011, abstr 3520

549 Glazer ES et al. Effectiveness of positron emission tomography for predicting chemotherapy response in colorectal cancer liver metastases. Arch Surg 2010; 145 (4): 340 - 345; discussion 345

550 Lubezky $N$ et al. The role and limitations of 18-fluoro-2-deoxy-D-glucose positron emission tomography (FDG-PET) scan and computerized tomography (CT) in restaging patients with hepatic colorectal metastases following neoadjuvant chemotherapy: comparison with operative and pathological findings. J Gastrointest Surg 2007; 11 (4): $472-478$

551 Spatz J et al. Neoadjuvant chemotherapy affects staging of colorectal liver metastasis - a comparison of PET, CT and intraoperative ultrasound. Int J Colorectal Dis 2011; 26 (2): 165-171

552 IQWIG. Positronenemissionstomographie (PET und PET/CT) bei rezidivierendem kolorektalen Karzinom - Vorbericht (vorläufige Nutzenbewertung); 2011

553 Patel S et al. Positron emission tomography/computed tomographic scans compared to computed tomographic scans for detecting colorectal liver metastases: a systematic review. Ann Surg 2011; 253 (4): 666- 671

554 Taylor RA, Akhurst TJ, Yuman F. Evaluation with positron emission tomography before hepatic resection for metastatic colorectal cancer improves survival in patients with a high clinical risk score; 2007 , Gastrointestinal Cancers Symposium, Abstract No: 240, 2007.

555 Adie $S$ et al. Resection of liver metastases from colorectal cancer: does preoperative chemotherapy affect the accuracy of PET in preoperative planning? ANZ J Surg 2009; 79 (5): 358 - 361

556 Levy $M$ et al. Tumor markers in staging and prognosis of colorectal carcinoma. Neoplasma 2008; 55 (2): 138 - 1342

557 Lewis MR et al. Metastatic colorectal adenocarcinoma involving the ovary with elevated serum CA125: a potential diagnostic pitfall. Gynecol Oncol 2007; 105 (2): 395 - 398

558 Chen CC et al. Is it reasonable to add preoperative serum level of CEA and CA19-9 to staging for colorectal cancer? J Surg Res 2005; 124 (2): $169-174$

559 Merkel S et al. The prognostic inhomogeneity in pT3 rectal carcinomas. Int J Colorectal Dis 2001; 16 (5): 298-304

560 Nagtegaal ID, Quirke P. What is the role for the circumferential margin in the modern treatment of rectal cancer? J Clin Oncol 2008; 26 (2): $303-312$

561 Bipat $S$ et al. Rectal cancer: local staging and assessment of lymph node involvement with endoluminal US, CT, and MR imaging - a meta-analysis. Radiology 2004; 232 (3): 773-783

562 Puli SR et al. Accuracy of endoscopic ultrasound to diagnose nodal invasion by rectal cancers: a meta-analysis and systematic review. Ann Surg Oncol 2009; 16 (5): 1255-1265

563 Ahmetoglu A et al. MDCT with multiplanar reconstruction in the preoperative local staging of rectal tumor. Abdom Imaging 2011; 36 (1): $31-37$

564 Kim CK et al. Preoperative staging of rectal cancer: accuracy of 3-Tesla magnetic resonance imaging. Eur Radiol 2006; 16 (5): 972 - 980

$565 \mathrm{Kim}$ YW et al. A prospective comparison study for predicting circumferential resection margin between preoperative MRI and whole mount sections in mid-rectal cancer: significance of different scan planes. Eur J Surg Oncol 2008; 34 (6): 648 - 654

$566 \mathrm{Kim}$ YW et al. Factors related to preoperative assessment of the circumferential resection margin and the extent of mesorectal invasion 
by magnetic resonance imaging in rectal cancer: a prospective comparison study. World J Surg 2009; 33 (9): 1952 - 1960

567 Matsuoka H et al. MRI diagnosis of mesorectal lymph node metastasis in patients with rectal carcinoma. what is the optimal criterion? Anticancer Res 2004; 24 (6): 4097-4101

568 Wolberink SV et al. Multislice CT as a primary screening tool for the prediction of an involved mesorectal fascia and distant metastases in primary rectal cancer: a multicenter study. Dis Colon Rectum 2009; 52 (5): 928 - 934

569 Purkayastha S et al. Magnetic resonance colonography vs. computed tomography colonography for the diagnosis of colorectal cancer: an indirect comparison. Colorectal Dis 2007; 9 (2): 100-111

570 Torzilli $G$ et al. Contrast-enhanced intraoperative ultrasonography during hepatectomies for colorectal cancer liver metastases. J Gastrointest Surg 2005; 9 (8): 1148 - 1153; discussion 1153-1154

571 Zirngibl H, Husemann B, Hermanek P. Intraoperative spillage of tumor cells in surgery for rectal cancer. Dis Colon Rectum 1990; 33 (7): 610-614

572 Cahill RA, Leroy J, Marescaux J. Could lymphatic mapping and sentinel node biopsy provide oncological providence for local resectional techniques for colon cancer? A review of the literature. BMC Surg 2008; 8: 17

573 Wiese $D$ et al. Ultrastaging of sentinel lymph nodes (SLNs) vs. nonSLNs in colorectal cancer - do we need both? Am J Surg 2010; 199 (3): $354-358$; discussion 358

574 Goligher JC. Incidence and pathology of carcinoma of the colon and rectum. In: Surgery of the anus, rectum and colon London: Balliére Tindall; 1984: 445

575 Toyota S, Ohta H, Anazawa S. Rationale for extent of lymph node dissection for right colon cancer. Dis Colon Rectum 1995; 38 (7): $705-711$

576 Tan $K Y$ et al. Distribution of the first metastatic lymph node in colon cancer and its clinical significance. Colorectal Dis 2010; 12 (1): 44-47

577 Lan YT et al. Significance of lymph node retrieval from the terminal ileum for patients with cecal and ascending colonic cancers. Ann Surg Oncol 2011; 18 (1): 146-152

578 Chang GJ et al. Lymph node evaluation and survival after curative resection of colon cancer: systematic review. J Natl Cancer Inst 2007; 99 (6): $433-441$

579 Le VoyerTE et al. Colon cancer survival is associated with increasing number of lymph nodes analyzed: a secondary survey of intergroup trial INT-0089. J Clin Oncol 2003; 21 (15): 2912-2919

580 Van Damme JP. Vascular anatomy in abdominal surgery. Stuttgart: Thieme; 1990

581 Kanemitsu Y et al. Survival benefit of high ligation of the inferior mesenteric artery in sigmoid colon or rectal cancer surgery. Br J Surg 2006; 93 (5): 609-615

582 Chin CC et al. Carcinoma obstruction of the proximal colon cancer and long-term prognosis - obstruction is a predictor of worse outcome in TNM stage II tumor. Int J Colorectal Dis 2010; 25 (7): 817 - 822

583 Hohenberger $W$ et al. Standardized surgery for colonic cancer: complete mesocolic excision and central ligation - technical notes and outcome. Colorectal Dis 2009; 11 (4): 354 - 364; discussion 364-365

584 West NP et al. Multicentre experience with extralevator abdominoperineal excision for low rectal cancer. Br J Surg 2010; 97 (4): 588 - 599

585 Bertelsen CA et al. Can the quality of colonic surgery be improved by standardization of surgical technique with complete mesocolic excision? Colorectal Dis 2011; 13 (10): 1123 - 1129

586 (NICE). N.I.f.C.E. Radiofrequency ablation for the treatment of colorectal metastases in the liver. London: 2011

$587 \mathrm{Kim} \mathrm{KH}$ et al. Comparative analysis of radiofrequency ablation and surgical resection for colorectal liver metastases. J Korean Surg Soc $2011 ; 81(1): 25-34$

588 Mulier S et al. Radiofrequency ablation versus resection for resectable colorectal liver metastases: time for a randomized trial? Ann Surg Oncol 2008; 15 (1): 144-157

589 (NICE). N.I.f.C.E. Selective internal radiation therapy for colorectal metastases in the liver. London: 2011

590 Townsend A, Price T, Karapetis $C$. Selective internal radiation therapy for liver metastases from colorectal cancer. Cochrane Database Syst Rev 2009; 4: CD007045

591 Christophi $C$ et al. Long-term survival of patients with unresectable colorectal liver metastases treated by percutaneous interstitial laser thermotherapy. World J Surg 2004; 28 (10): 987-994
592 Wietzke-Braun P et al. Quality of life and outcome of ultrasoundguided laser interstitial thermo-therapy for non-resectable liver metastases of colorectal cancer. Eur J Gastroenterol Hepatol 2004; 16 (4): $389-395$

593 Abcarian H, Pearl RK. Simple technique for high ligation of the inferior mesenteric artery and vein. Dis Colon Rectum 1991; 34 (12): 1138

594 Buunen $M$ et al. Level of arterial ligation in total mesorectal excision (TME): an anatomical study. Int J Colorectal Dis 2009; 24 (11): $1317-1320$

595 Pezim ME, Nicholls RJ. Survival after high or low ligation of the inferior mesenteric artery during curative surgery for rectal cancer. Ann Surg $1984 ; 200$ (6): $729-733$

596 Surtees $P$, Ritchie JK, Phillips RK. High versus low ligation of the inferior mesenteric artery in rectal cancer. Br J Surg 1990; 77 (6): 618-621

597 Slanetz CA Jr, Grimson R. Effect of high and intermediate ligation on survival and recurrence rates following curative resection of colorectal cancer. Dis Colon Rectum 1997; 40 (10): 1205-1218; discussion 1218-1219

598 Lange MM et al. Level of arterial ligation in rectal cancer surgery: low tie preferred over high tie. A review. Dis Colon Rectum 2008; 51 (7): $1139-1145$

599 Heald RJ, Husband EM, Ryall RD. The mesorectum in rectal cancer surgery - the clue to pelvic recurrence? Br J Surg 1982; 69 (10): $613-616$

600 Bokey EL et al. Local recurrence after curative excision of the rectum for cancer without adjuvant therapy: role of total anatomical dissection. Br J Surg 1999; 86 (9): 1164-1170

601 Hermanek PJ. Multiviszerale Resektion beim kolorektalen Karzinom. Erfahrungen der SGKRK-Studie. Langenbecks Arch Surg 1992; Kongressband: $95-100$

602 Havenga $\mathrm{K}$ et al. Male and female sexual and urinary function after total mesorectal excision with autonomic nerve preservation for carcinoma of the rectum. J Am Coll Surg 1996; 182 (6): 495-502

603 Enker WE et al. Abdominoperineal resection via total mesorectal excision and autonomic nerve preservation for low rectal cancer. World J Surg 1997; 21 (7): $715-720$

604 Georgiou $P$ et al. Extended lymphadenectomy versus conventional surgery for rectal cancer: a meta-analysis. Lancet Oncol 2009; 10 (11): $1053-1062$

605 Gohl J, Hohenberger W, Merkel S. Lymph node dissection in rectal carcinoma: TME and what else? Onkologie 2009; 32 (1): 57 -61

606 Mortenson MM et al. Total mesorectal excision and pelvic node dissection for rectal cancer: an appraisal. Surg Oncol Clin N Am 2007; 16 (1): $177-197$

607 Koch $M$ et al. Is the lateral lymph node compartment relevant? Recent Results Cancer Res 2005; 165: 40-45

608 Scott $N$ et al. Total mesorectal excision and local recurrence: a study of tumour spread in the mesorectum distal to rectal cancer. Br J Surg 1995; 82 (8): 1031 - 1033

609 Reynolds JV et al. Pathological evidence in support of total mesorectal excision in the management of rectal cancer. Br J Surg 1996; 83 (8): $1112-1115$

610 Hida J et al. Lymph node metastases detected in the mesorectum distal to carcinoma of the rectum by the clearing method: justification of total mesorectal excision. J Am Coll Surg 1997; 184 (6): $584-588$

611 Zaheer $S$ et al. Surgical treatment of adenocarcinoma of the rectum. Ann Surg 1998; 227 (6): 800-811

612 Leong AF. Selective total mesorectal excision for rectal cancer. Dis Colon Rectum 2000; 43 (9): 1237-1240

613 Heald RJ, Karanjia ND. Results of radical surgery for rectal cancer. World J Surg 1992; 16 (5): $848-857$

614 Enker WE. Total mesorectal excision - the new golden standard of surgery for rectal cancer. Ann Med 1997; 29 (2): 127-133

615 Lazorthes F et al. Distal intramural spread of carcinoma of the rectum correlated with lymph nodal involvement. Surg Gynecol Obstet 1990; 170 (1): $45-48$

616 Kwok SP et al. Prospective analysis of the distal margin of clearance in anterior resection for rectal carcinoma. Br J Surg 1996; 83 (7): 969 972

617 Andreola $S$ et al. Distal intramural spread in adenocarcinoma of the lower third of the rectum treated with total rectal resection and coloanal anastomosis. Dis Colon Rectum 1997; 40 (1): 25 - 29

618 Goldstein NS, Soman A, Sacksner J. Disparate surgical margin lengths of colorectal resection specimens between in vivo and in vitro mea- 
surements. The effects of surgical resection and formalin fixation on organ shrinkage. Am J Clin Pathol 1999; 111 (3): 349-351

619 Kiran RP, Lian L, Lavery IC. Does a subcentimeter distal resection margin adversely influence oncologic outcomes in patients with rectal cancer undergoing restorative proctectomy? Dis Colon Rectum 2011; 54 (2): $157-163$

620 Rutkowski A et al. Distal bowel surgical margin shorter than $1 \mathrm{~cm}$ after preoperative radiation for rectal cancer: is it safe? Ann Surg Oncol 2008; 15 (11): $3124-3131$

621 Brown CJ, Fenech DS, McLeod RS. Reconstructive techniques after rectal resection for rectal cancer. Cochrane Database Syst Rev 2008; 2: CD006040

622 Fazio VW et al. A randomized multicenter trial to compare long-term functional outcome, quality of life, and complications of surgical procedures for low rectal cancers. Ann Surg 2007; 246 (3): 481-488; discussion 488-490

623 Liao C et al. Meta-analysis of the colon J-pouch vs. transverse coloplasty pouch after anterior resection for rectal cancer. Colorectal Dis 2010; 12 (7): 624-631

624 Siddiqui MR et al. A meta-analysis comparing side to end with colonic J-pouch formation after anterior resection for rectal cancer. Tech Coloproctol 2010; 14 (2): 113-123

625 Hida J et al. Functional outcome after low anterior resection with low anastomosis for rectal cancer using the colonic J-pouch. Prospective randomized study for determination of optimum pouch size. Dis Colon Rectum 1996; 39 (9): 986-991

626 Hohenberger $W$ et al. Neoadjuvant radiochemotherapy for rectal cancer. Chirurg 2009; 80 (4): 294-302

627 Artioukh DY. Controversial aspects of rectal cancer surgery following preoperative chemoradiation. Colorectal Dis 2010; 12 (Suppl 2): $25-29$

628 Habr-Gama A et al. Operative versus nonoperative treatment for stage 0 distal rectal cancer following chemoradiation therapy: longterm results. Ann Surg 2004; 240 (4): 711 - 717; discussion 717-718

629 Tekkis PP et al. Comparison of circumferential margin involvement between restorative and nonrestorative resections for rectal cancer. Colorectal Dis 2005; 7 (4): 369-374

630 Nagtegaal ID et al. Low rectal cancer: a call for a change of approach in abdominoperineal resection. J Clin Oncol 2005; 23 (36): 9257-9264

631 Nisar PJ, Scott HJ. Myocutaneous flap reconstruction of the pelvis after abdominoperineal excision. Colorectal Dis 2009; 11 (8): 806-816

632 Montedori A et al. Covering ileo- or colostomy in anterior resection for rectal carcinoma. Cochrane Database Syst Rev 2010; 5: CD006878

633 Lindgren $R$ et al. What is the risk for a permanent stoma after low anterior resection of the rectum for cancer? A six-year follow-up of a multicenter trial. Dis Colon Rectum 2011; 54 (1): 41 - 47

634 Klink CD et al. Protective ileostoma versus protective transverse stoma. What evidence is available? Chirurg 2010; 81 (11): 974-977

635 Rondelli $F$ et al. Loop ileostomy versus loop colostomy for fecal diversion after colorectal or coloanal anastomosis: a meta-analysis. Int J Colorectal Dis 2009; 24 (5): 479-488

636 Bass EM et al. Does preoperative stoma marking and education by the enterostomal therapist affect outcome? Dis Colon Rectum 1997; 40 (4): $440-442$

637 Millan $M$ et al. Preoperative stoma siting and education by stomatherapists of colorectal cancer patients: a descriptive study in twelve Spanish colorectal surgical units. Colorectal Dis 2010; 12 (7): e88e92

638 Chaudhri $S$ et al. Preoperative intensive, community-based vs. traditional stoma education: a randomized, controlled trial. Dis Colon Rectum 2005; 48 (3): 504-509

639 Hermanek P, Marzoli GP. Lokale Therapie des Rektumkarzinoms. Verfahren in kurativer Intention. Berlin Heidelberg Ney York Tokyo: Springer; 1994

640 Winde G et al. Surgical cure for early rectal carcinomas (T1). Transanal endoscopic microsurgery vs. anterior resection. Dis Colon Rectum 1996; 39 (9): $969-976$

641 Sengupta S, Tjandra JJ. Local excision of rectal cancer: what is the evidence? Dis Colon Rectum 2001; 44 (9): 1345-1361

642 Matzel KE, Merkel S, Hohenberger W. Lokale Therapieprinzipien beim Rektumkarzinom. Chirurg 2003; 74 (10): 897-904

643 Ptok $\mathrm{H}$ et al. Oncological outcome of local vs. radical resection of lowrisk pT1 rectal cancer. Arch Surg 2007; 142 (7): 649-655; discussion 656
644 You YN et al. Is the increasing rate of local excision for stage I rectal cancer in the United States justified? A nationwide cohort study from the National Cancer Database. Ann Surg 2007; 245 (5): $726-$ 733

645 Moore JS et al. Transanal endoscopic microsurgery is more effective than traditional transanal excision for resection of rectal masses. Dis Colon Rectum 2008; 51 (7): 1026 - 1030; discussion 1030-1031

646 Sgourakis $G$ et al. Transanal endoscopic microsurgery for T1 and T2 rectal cancers: a meta-analysis and meta-regression analysis of outcomes. Am Surg 2011; 77 (6): 761 - 772

647 Bach SP et al. A predictive model for local recurrence after transanal endoscopic microsurgery for rectal cancer. Br J Surg 2009; 96 (3): $280-290$

648 Glimelius B, Pahlman L, Cervantes A. Rectal cancer: ESMO Clinical Practice Guidelines for diagnosis, treatment and follow-up. Ann Oncol 2010; 21 (Suppl 5): v82 - v86

649 Suppiah $A$ et al. Transanal endoscopic microsurgery in early recta cancer: time for a trial? Colorectal Dis 2008; 10 (4): 314-327; discussion 327-329

650 Valentini $V$ et al. Multidisciplinary Rectal Cancer Management: 2nd European Rectal Cancer Consensus Conference (EURECA-CC2). Radiother Oncol 2009; 92 (2): 148-163

651 Hahnloser D et al. Immediate radical resection after local excision of rectal cancer: an oncologic compromise? Dis Colon Rectum 2005; 48 (3): $429-437$

652 Schwenk $W$ et al. Short term benefits for laparoscopic colorectal resection. Cochrane Database Syst Rev 2005; 3: CD003145

653 Liang $Y$ et al. Laparoscopic versus open colorectal resection for cancer: a meta-analysis of results of randomized controlled trials on recurrence. Eur J Surg Oncol 2008; 34 (11): 1217-1224

654 Kuhry E et al. Long-term results of laparoscopic colorectal cancer resection. Cochrane Database Syst Rev 2008; 2: CD003432

655 Guillou PJ et al. Short-term endpoints of conventional versus laparoscopic-assisted surgery in patients with colorectal cancer (MRC CLASICC trial): multicentre, randomised controlled trial. Lancet 2005; 365 (9472): $1718-1726$

656 Jayne DG et al. Five-year follow-up of the Medical Research Council CLASICC trial of laparoscopically assisted versus open surgery for colorectal cancer. Br J Surg 2010; 97 (11): 1638 - 1645

657 Kwak JM et al. Robotic vs. laparoscopic resection of rectal cancer: short-term outcomes of a case-control study. Dis Colon Rectum 2011; 54 (2): $151-156$

658 Pigazzi A et al. Multicentric study on robotic tumor-specific mesorectal excision for the treatment of rectal cancer. Ann Surg Oncol 2010; 17 (6): $1614-1620$

659 Jacquet $P$, Sugarbaker $P H$. Clinical research methodologies in diagnosis and staging of patients with peritoneal carcinomatosis. Cancer Treat Res 1996; 82: 359-374

660 Verwaal VJ et al. Randomized trial of cytoreduction and hyperthermic intraperitoneal chemotherapy versus systemic chemotherapy and palliative surgery in patients with peritoneal carcinomatosis of colorectal cancer. J Clin Oncol 2003; 21 (20): 3737-3743

661 Verwaal VJ et al. 8-year follow-up of randomized trial: cytoreduction and hyperthermic intraperitoneal chemotherapy versus systemic chemotherapy in patients with peritoneal carcinomatosis of colorectal cancer. Ann Surg Oncol 2008; 15 (9): 2426-2432

$662 \mathrm{Cao} C$ et al. A systematic review and meta-analysis of cytoreductive surgery with perioperative intraperitoneal chemotherapy for peritoneal carcinomatosis of colorectal origin. Ann Surg Oncol 2009; 16 (8): $2152-2165$

663 Khot UP et al. Systematic review of the efficacy and safety of colorectal stents. Br J Surg 2002; 89 (9): 1096 - 1102

664 Stillwell AP, Buettner PG, Ho YH. Meta-analysis of survival of patients with stage IV colorectal cancer managed with surgical resection versus chemotherapy alone. World J Surg 2010; 34 (4): 797-807

665 Poultsides GA et al. Outcome of primary tumor in patients with synchronous stage IV colorectal cancer receiving combination chemotherapy without surgery as initial treatment. J Clin Oncol 2009; 27 (20): $3379-3384$

666 Hermanek P, Guggenmoos-Holzmann I, Gall FP. Prognostic factors in rectal carcinoma. A contribution to the further development of tumor classification. Dis Colon Rectum 1989; 32 (7): 593-599

667 Hermanek $P$, Hohenberger $W$. The importance of volume in colorectal cancer surgery. Eur J Surg Oncol 1996; 22 (3): 213-215 
668 Compton CC. Pathology report in colon cancer: what is prognostically important? Dig Dis 1999; 17 (2): $67-79$

669 Wong JH et al. Number of nodes examined and staging accuracy in colorectal carcinoma. J Clin Oncol 1999; 17 (9): 2896-2900

670 Compton $C$ et al. American Joint Committee on Cancer Prognostic Factors Consensus Conference: Colorectal Working Group. Cancer 2000; 88 (7): $1739-1757$

671 Compton CC. Updated protocol for the examination of specimens from patients with carcinomas of the colon and rectum, excluding carcinoid tumors, lymphomas, sarcomas, and tumors of the vermiform appendix: a basis for checklists. Cancer Committee. Arch Pathol Lab Med 2000; 124 (7): 1016-1025

672 Hermanek P. Methodik der histopathologischen Untersuchung von Resektionen kolorektaler Karzinome. Chir Gastroenterol 2000; 16: 255-259

673 Hermanek $P$. Lymph nodes and malignant tumors. Zentralbl Chir 2000; 125 (10): 790 - 795

674 Junginger THP, Klimpfinger M. Klassifikation maligner Tumoren des Gastrointestinaltraktes I. Berlin Heidelberg New York: Springer Verlag; 2002

675 Wittekind $C$ et al. TNM residual tumor classification revisited. Cancer 2002; 94 (9): $2511-2516$

676 Leibl S, Tsybrovskyy O, Denk $H$. How many lymph nodes are necessary to stage early and advanced adenocarcinoma of the sigmoid colon and upper rectum? Virchows Arch 2003; 443 (2): 133-138

677 Sobin LH. TNM, sixth edition: new developments in general concepts and rules. Semin Surg Oncol 2003; 21 (1): 19-22

678 Dworak O, Keilholz L, Hoffmann A. Pathological features of rectal cancer after preoperative radiochemotherapy. Int J Colorectal Dis 1997; $12(1): 19-23$

679 Wright CM et al. Prognostic significance of extensive microsatellite instability in sporadic clinicopathological stage C colorectal cancer. Br J Surg 2000; 87 (9): 1197-1202

680 Popat S, Hubner R, Houlston RS. Systematic review of microsatellite instability and colorectal cancer prognosis. J Clin Oncol 2005; 23 (3): 609-618

681 Benatti Pet al. Microsatellite instability and colorectal cancer prognosis. Clin Cancer Res 2005; 11 (23): 8332-8340

682 Malesci A et al. Reduced likelihood of metastases in patients with microsatellite-unstable colorectal cancer. Clin Cancer Res 2007; 13 (13): $3831-3839$

683 Leopoldo $S$ et al. Two subtypes of mucinous adenocarcinoma of the colorectum: clinicopathological and genetic features. Ann Surg Oncol 2008; 15 (5): 1429-1439

684 Lanza $G$ et al. Medullary-type poorly differentiated adenocarcinoma of the large bowel: a distinct clinicopathologic entity characterized by microsatellite instability and improved survival. J Clin Oncol 1999; 17 (8): 2429-2438

685 Parc $Y$ et al. Prognostic significance of microsatellite instability determined by immunohistochemical staining of MSH2 and MLH1 in sporadic T3NOM0 colon cancer. Gut 2004; 53 (3): 371 - 375

686 Gafa R et al. Sporadic colorectal adenocarcinomas with high-frequency microsatellite instability. Cancer 2000; 89 (10): 2025-2037

687 Lanza G et al. Immunohistochemical pattern of MLH1 / MSH2 expression is related to clinical and pathological features in colorectal adenocarcinomas with microsatellite instability. Mod Pathol 2002; 15 (7): $741-749$

688 Lindor NM et al. Immunohistochemistry versus microsatellite instability testing in phenotyping colorectal tumors. J Clin Oncol 2002; 20 (4): $1043-1048$

689 Overbeek LI et al. Interpretation of immunohistochemistry for mismatch repair proteins is only reliable in a specialized setting. Am J Surg Pathol 2008; 32 (8): 1246-1251

690 Prandi $M$ et al. Prognostic evaluation of stage B colon cancer patients is improved by an adequate lymphadenectomy: results of a secondary analysis of a large scale adjuvant trial. Ann Surg 2002; 235 (4): $458-463$

691 Ogino $S$ et al. Negative lymph node count is associated with survival of colorectal cancer patients, independent of tumoral molecular alterations and lymphocytic reaction. Am J Gastroenterol 2010; 105 (2): $420-433$

692 Swanson RS et al. The prognosis of T3N0 colon cancer is dependent on the number of lymph nodes examined. Ann Surg Oncol 2003; 10 (1): $65-71$
693 Carloss $\mathrm{H}$ et al. The impact of number of lymph nodes removed on five-year survival in stage II colon and rectal cancer. J Ky Med Assoc 2004; 102 (8): 345 - 347

694 Fazio L et al. Association between colonic screening, subject characteristics, and stage of colorectal cancer. Am J Gastroenterol 2005; 100 (11): $2531-2539$

695 Wong SL et al. Hospital lymph node examination rates and survival after resection for colon cancer. JAMA 2007; 298 (18): 2149-2154

696 Qiu HB et al. Ratio of metastatic to resected lymph nodes enhances to predict survival in patients with stage III colorectal cancer. Ann Surg Oncol 2011; 18 (6): 1568 - 1574

697 Wang J et al. Should total number of lymph nodes be used as a quality of care measure for stage III colon cancer? Ann Surg 2009; 249 (4): $559-563$

698 Chen SL et al. Lymph node ratio as a quality and prognostic indicator in stage III colon cancer. Ann Surg 2011; 253 (1): 82 - 87

$699 \mathrm{Ha} \mathrm{YH}$ et al. Influence of preoperative chemoradiotherapy on the number of lymph nodes retrieved in rectal cancer. Ann Surg 2010 $252(2): 336-340$

700 West NP et al. Complete mesocolic excision with central vascular ligation produces an oncologically superior specimen compared with standard surgery for carcinoma of the colon. J Clin Oncol 2010; 28 (2): $272-278$

701 West NP et al. Pathology grading of colon cancer surgical resection and its association with survival: a retrospective observational study. Lancet Oncol 2008; 9 (9): 857-865

702 Quirke $P$ et al. Effect of the plane of surgery achieved on local recurrence in patients with operable rectal cancer: a prospective study using data from the MRC CR07 and NCIC-CTG CO16 randomised clinical trial. Lancet 2009; 373 (9666): $821-828$

703 Tilney HS et al. The influence of circumferential resection margins on long-term outcomes following rectal cancer surgery. Dis Colon Rectum 2009; 52 (10): $1723-1729$

704 Bernstein TE et al. Circumferential resection margin as a prognostic factor in rectal cancer. Br J Surg 2009; 96 (11): 1348-1357

705 West NP et al. Evidence of the oncologic superiority of cylindrical abdominoperineal excision for low rectal cancer. J Clin Oncol 2008 26 (21): $3517-3522$

706 Leite JS et al. Clinical significance of macroscopic completeness of mesorectal resection in rectal cancer. Colorectal Dis 2011; 13 (4): 381 386

707 Kreienberg $R$ et al. Interdisziplinäre S3-Leitlinie für die Diagnostik, Therapie und Nachsorge des Mammakarzinoms. Informationszentrum für Standards in der Onkologie (ISTO), Deutsche Krebsgesellschaft e.V; 2008, 1. Aktualisierung

708 Edwards AG, Hailey S, Maxwell M. Psychological interventions for women with metastatic breast cancer. Cochrane Database Syst Rev 2004; 2: CD004253

709 Sheard T, Maguire P. The effect of psychological interventions on anxiety and depression in cancer patients: results of two meta-analyses. Br J Cancer 1999; 80 (11): 1770 - 1780

710 Keller $M$ et al. Recognition of distress and psychiatric morbidity in cancer patients: a multi-method approach. Ann Oncol 2004; 15 (8): $1243-1249$

711 Sharpe $M$ et al. Major depression in outpatients attending a regional cancer centre: screening and unmet treatment needs. Br J Cancer 2004; 90 (2): $314-320$

712 Singer S, Das-Munshi J, Brahler E. Prevalence of mental health conditions in cancer patients in acute care - a meta-analysis. Ann Oncol 2010; 21 (5): 925-930

713 Zabora J et al. The prevalence of psychological distress by cancer site. Psychooncology 2001; 10 (1): 19-28

714 Jansen $L$ et al. Health-related quality of life during the 10 years after diagnosis of colorectal cancer: a population-based study. J Clin Oncol 2011; 29 (24): $3263-3269$

715 Lynch BM et al. Describing and predicting psychological distress after colorectal cancer. Cancer 2008; 112 (6): 1363-1370

716 Miovic M, Block S. Psychiatric disorders in advanced cancer. Cancer 2007; 110 (8): $1665-1676$

717 Ramsey SD et al. Quality of life in survivors of colorectal carcinoma. Cancer 2000; 88 (6): 1294-1303

718 Zettl S, Hartlapp J (eds.) Sexualstörungen durch Krankheit und Therapie. Heidelberg: ed. Springer; 1997: S. 59

719 Fallowfield $L$ et al. Psychiatric morbidity and its recognition by doctors in patients with cancer. Br J Cancer 2001; 84 (8): 1011 - 1015 
720 Sollner $W$ et al. How successful are oncologists in identifying patient distress, perceived social support, and need for psychosocial counselling? Br J Cancer 2001; 84 (2): 179-185

721 Ell $\mathrm{K}$ et al. Randomized controlled trial of collaborative care management of depression among low-income patients with cancer. J Clin Oncol 2008; 26 (27): $4488-4496$

722 Strong $V$ et al. Management of depression for people with cancer (SMaRT oncology 1): a randomised trial. Lancet 2008; 372 (9632): $40-48$

723 Herschbach $P$, Weis J (eds.) Screeningverfahren in der Psychoonkologie. Berlin: Deutsche Krebsgesellschaft; 2008

724 NHMRC. Clinical practice guidelines for the psychosocial care of adults with cancer; 2003: 1 -237

725 Turner J et al. Clinical practice guidelines for the psychosocial care of adults with cancer. Psychooncology 2005; 14 (3): 159-173

726 Weis J et al. Psychoonkologie: Konzepte und Aufgaben einer jungen Fachdisziplin. Onkologe 2007; 13 (2): 185 - 194

727 Edwards AG, Hulbert-Williams N, Neal RD. Psychological interventions for women with metastatic breast cancer. Cochrane Database Syst Rev 2008; 3: CD004253

728 Faller $\mathrm{H}$ et al. Effects of psychooncological interventions on emotional distress and quality of life in adult cancer patients: systematic review and meta-analysis. Nat J Cancer in press.

729 Goedendorp MM et al. Psychosocial interventions for reducing fatigue during cancer treatment in adults. Cochrane Database Syst Rev 2009; 1: CD006953

730 Jacobsen PB, Jim HS. Psychosocial interventions for anxiety and depression in adult cancer patients: achievements and challenges. CA Cancer J Clin 2008; 58 (4): 214-230

731 Luebbert K, Dahme B, Hasenbring $M$. The effectiveness of relaxation training in reducing treatment-related symptoms and improving emotional adjustment in acute non-surgical cancer treatment: a meta-analytical review. Psychooncology 2001; 10 (6): 490-502

732 Osborn RL, Demoncada AC, Feuerstein M. Psychosocial interventions for depression, anxiety, and quality of life in cancer survivors: metaanalyses. Int J Psychiatry Med 2006; 36 (1): 13-34

733 Rehse B, Pukrop R. Effects of psychosocial interventions on quality of life in adult cancer patients: meta analysis of 37 published controlled outcome studies. Patient Educ Couns 2003; 50 (2): 179-186

734 Uitterhoeve RJ et al. Psychosocial interventions for patients with advanced cancer - a systematic review of the literature. $\mathrm{Br}$ J Cancer 2004; 91 (6): 1050 - 1062

735 Williams S, Dale J. The effectiveness of treatment for depression/depressive symptoms in adults with cancer: a systematic review. $\mathrm{Br}$ J Cancer 2006; 94 (3): 372 - 390

736 Iwashyna TJ, Lamont EB. Effectiveness of adjuvant fluorouracil in clinical practice: a population-based cohort study of elderly patients with stage III colon cancer. J Clin Oncol 2002; 20 (19): 3992 - 3998

737 Lonardi S, Stefani M, Jirillo A et al. Benefit of fluorouracil and folinic acid adjuvant in colon cancer. Journal of Clinical Oncology 2006, ASCO Annual Meeting Proceedings (Post-Meeting Edition) 24 (Suppl 18): Abstract 13564

738 Sargent DJ et al. A pooled analysis of adjuvant chemotherapy for resected colon cancer in elderly patients. N Engl J Med 2001; 345 (15): $1091-1097$

739 Popescu RA et al. Adjuvant or palliative chemotherapy for colorectal cancer in patients 70 years or older. J Clin Oncol 1999; 17 (8): $2412-2418$

740 Goldberg RM, Tabah-Fisch I, Bleiberg H et al. Pooled Analysis of Safety and Efficacy of Oxaliplatin Plus Fluorouracil/Leucovorin Administered Bimonthly in Elderly Patients With Colorectal Cancer. Journal of Clinical Oncology 2006; 24 (25): 4085 -4091

741 Fata F et al. Efficacy and toxicity of adjuvant chemotherapy in elderly patients with colon carcinoma: a 10-year experience of the Geisinger Medical Center. Cancer 2002; 94 (7): 1931 - 1938

742 Francini $G$ et al. Folinic acid and 5-fluorouracil as adjuvant chemotherapy in colon cancer. Gastroenterology 1994; 106 (4): 899-906

743 O'Connell MJ et al. Controlled trial of fluorouracil and low-dose leucovorin given for 6 months as postoperative adjuvant therapy for colon cancer. J Clin Oncol 1997; 15 (1): 246-250

744 Dube S, Heyen F, Jenicek M. Adjuvant chemotherapy in colorectal carcinoma: results of a meta-analysis. Dis Colon Rectum 1997; 40 (1): $35-41$
745 Gill S et al. Pooled analysis of fluorouracil-based adjuvant therapy for stage II and III colon cancer: who benefits and by how much? J Clin Oncol 2004; 22 (10): 1797 - 1806

746 Efficacy of adjuvant fluorouracil and folinic acid in colon cancer. International Multicentre Pooled Analysis of Colon Cancer Trials (IMPACT) investigators. Lancet 1995; 345 (8955): 939-944

747 Figueredo A et al. Adjuvant therapy for stage II colon cancer after complete resection. Provincial Gastrointestinal Disease Site Group. Cancer Prev Control 1997; 1 (5): 379-392

748 Efficacy of adjuvant fluorouracil and folinic acid in B2 colon cancer. International Multicentre Pooled Analysis of B2 Colon Cancer Trials (IMPACT B2) Investigators. J Clin Oncol 1999; 17 (5): 1356-1363

749 Mamounas E et al. Comparative efficacy of adjuvant chemotherapy in patients with Dukes' B versus Dukes' C colon cancer: results from four National Surgical Adjuvant Breast and Bowel Project adjuvant studies (C-01, C-02, C-03, and C-04). J Clin Oncol 1999; 17 (5): 1349-1355

750 Benson $A B$ 3rd et al. American Society of Clinical Oncology recommendations on adjuvant chemotherapy for stage II colon cancer. J Clin Oncol 2004; 22 (16): 3408 - 3419

751 Quasar Collaborative G. et al. Adjuvant chemotherapy versus observation in patients with colorectal cancer: a randomised study. Lancet 2007; 370 (9604): 2020-2029

752 Gramont A, Boni C, Navarro $M$ et al. Oxaliplatin/5FU/LV in adjuvant colon cancer: Updated efficacy results of the MOSAIC trial, including survival, with a median follow-up of six years. Journal of Clinical Oncology 2007, ASCO Annual Meeting Proceedings (Post-Meeting Edition) 25 (Suppl 18): Abstract 4007

753 Moertel CG et al. Intergroup study of fluorouracil plus levamisole as adjuvant therapy for stage II/Dukes' B2 colon cancer. J Clin Oncol 1995; 13 (12): 2936-2943

754 Taal BG, Van Tinteren H, Zoetmulder FA. Adjuvant 5FU plus levamisole in colonic or rectal cancer: improved survival in stage II and III. Br J Cancer 2001; 85 (10): 1437-1443

755 Schrag $D$ et al. Adjuvant chemotherapy use for Medicare beneficiaries with stage II colon cancer. J Clin Oncol 2002; 20 (19): 3999 - 4005

756 Merkel $S$ et al. High-risk groups of patients with Stage II colon carcinoma. Cancer 2001; 92 (6): 1435-1443

757 Petersen VC et al. Identification of objective pathological prognostic determinants and models of prognosis in Dukes' B colon cancer. Gut 2002; 51 (1): $65-69$

758 Morris $M$ et al. Population-based study of prognostic factors in stage II colonic cancer. Br J Surg 2006; 93 (7): 866 - 871

759 Burdy $G$ et al. Identifying patients with T3-T4 node-negative colon cancer at high risk of recurrence. Dis Colon Rectum 2001; 44 (11): $1682-1688$

760 Jestin P et al. Emergency surgery for colonic cancer in a defined population. Br J Surg 2005; 92 (1): $94-100$

761 McArdle CS, McMillan DC, Hole DJ. The impact of blood loss, obstruction and perforation on survival in patients undergoing curative resection for colon cancer. Br J Surg 2006; 93 (4): 483 - 488

762 Caplin S et al. For patients with Dukes' B (TNM Stage II) colorectal carcinoma, examination of six or fewer lymph nodes is related to poor prognosis. Cancer 1998; 83 (4): 666-672

763 George $S$ et al. Will Rogers revisited: prospective observational study of survival of 3592 patients with colorectal cancer according to number of nodes examined by pathologists. Br J Cancer 2006; 95 (7): $841-847$

764 Chen SL, Bilchik AJ. More extensive nodal dissection improves survival for stages I to III of colon cancer: a population-based study. Ann Surg 2006; 244 (4): $602-610$

765 Moertel CG et al. Levamisole and fluorouracil for adjuvant therapy of resected colon carcinoma. N Engl J Med 1990; 322 (6): 352 - 358

766 Hermanek P, Hutter RV, Sobin LH. Prognostic grouping: the next step in tumor classification. J Cancer Res Clin Oncol 1990; 116 (5): 513 516

767 Martinez-Lopez E et al. Allelic loss on chromosome $18 \mathrm{q}$ as a prognostic marker in stage II colorectal cancer. Gastroenterology 1998; 114 (6): $1180-1187$

768 Ogunbiyi $O A$ et al. Confirmation that chromosome $18 \mathrm{q}$ allelic loss in colon cancer is a prognostic indicator. J Clin Oncol 1998; 16 (2): $427-433$

769 Jernvall $P$ et al. Loss of heterozygosity at $18 q 21$ is indicative of recurrence and therefore poor prognosis in a subset of colorectal cancers. Br J Cancer 1999; 79 (5): 903 -908 
770 Font A et al. Prognostic value of K-ras mutations and allelic imbalance on chromosome $18 \mathrm{q}$ in patients with resected colorectal cancer. Dis Colon Rectum 2001; 44 (4): 549 - 557

771 Diep CB et al. Genetic tumor markers with prognostic impact in Dukes' stages B and C colorectal cancer patients. J Clin Oncol 2003; 21 (5): $820-829$

772 Carethers JM et al. Prognostic significance of allelic lost at chromosome 18q21 for stage II colorectal cancer. Gastroenterology 1998; 114 (6): $1188-1195$

773 Lanza G et al. Immunohistochemical test for MLH1 and MSH2 expression predicts clinical outcome in stage II and III colorectal cancer patients. J Clin Oncol 2006; 24 (15): 2359-2367

774 Sinicrope FA et al. Prognostic impact of microsatellite instability and DNA ploidy in human colon carcinoma patients. Gastroenterology 2006; 131 (3): $729-737$

775 Araujo SE et al. DNA ploidy status and prognosis in colorectal cancer: a meta-analysis of published data. Dis Colon Rectum 2007; 50 (11): $1800-1810$

776 Ribic CM et al. Tumor microsatellite-instability status as a predictor of benefit from fluorouracil-based adjuvant chemotherapy for colon cancer. N Engl J Med 2003; 349 (3): 247 - 257

777 Elsaleh $H$, Iacopetta $B$. Microsatellite instability is a predictive marker for survival benefit from adjuvant chemotherapy in a population-based series of stage III colorectal carcinoma. Clin Colorectal Cancer 2001; 1 (2): $104-109$

778 Andre Tet al. Oxaliplatin, fluorouracil, and leucovorin as adjuvant treatment for colon cancer. N Engl J Med 2004; 350 (23): 2343 - 2351

779 Kuebler JP et al. Oxaliplatin combined with weekly bolus fluorouracil and leucovorin as surgical adjuvant chemotherapy for stage II and IIl colon cancer: results from NSABP C-07. J Clin Oncol 2007; 25 (16): 2198-2204

780 Schmoll HJ et al. Phase III trial of capecitabine plus oxaliplatin as adjuvant therapy for stage III colon cancer: a planned safety analysis in 1,864 patients. J Clin Oncol 2007; 25 (1): $102-109$

781 Van Cutsem E, Labianca R, Hossfeld D et al. PETACC 3, Randomized phase III trial comparing infused irinotecan/5-fluorouracil (5-FU)/ folinic acid (IF) versus 5-FU/FA $(F)$ in stage III colon cancer patients (pts). (PETACC 3). Journal of Clinical Oncology 2005, ASCO Annual Meeting Proceedings Abstract LBA8

782 Ychou M, Raoul J, Douillard J et al. , for the GI Group of the FNCLCC and the FFCD A phase III randomized trial of LV5FU2+CPT-11 vs. LV5FU2 alone in adjuvant high risk colon cancer (FNCLCC Accord02/ FFCD9802). Journal of Clinical Oncology 2005, ASCO Annual Meeting Proceedings 2005 23: Abstract 3502 (Suppl 16, Part I of II):

783 Saltz LB et al. Irinotecan fluorouracil plus leucovorin is not superior to fluorouracil plus leucovorin alone as adjuvant treatment for stage III colon cancer: results of CALGB 89803. J Clin Oncol 2007; 25 (23): $3456-3461$

784 Twelves $C$ et al. Capecitabine as adjuvant treatment for stage III colon cancer. N Engl J Med 2005; 352 (26): 2696 - 2704

785 Lembersky BC et al. Oral uracil and tegafur plus leucovorin compared with intravenous fluorouracil and leucovorin in stage II and III carcinoma of the colon: results from National Surgical Adjuvant Breast and Bowel Project Protocol C-06. J Clin Oncol 2006; 24 (13): 2059 2064

786 Sakamoto J et al. Efficacy of oral adjuvant therapy after resection of colorectal cancer: 5-year results from three randomized trials. J Clin Oncol 2004; 22 (3): 484-492

787 Carrato A, Köhne C, Bedenne L et al. for AIO/CAO, FFCD, TTD, EORTC olinic acid modulated bolus 5-FU or infusional 5-FU for adjuvant treatment of patients of UICC stage III colon cancer: Preliminary analysis of the PETACC-2-study. F. Journal of Clinical Oncology 2006, ASCO Annual Meeting Proceedings (Post-Meeting Edition) 2006; 24 (Suppl 18): Abstract 3563

788 Andre T, Quinaux E, Louvet $C$ et al. GERCOR. Updated results at 6 year of the GERCOR C96.1 phase III study comparing LV5FU2 to monthly 5FU-leucovorin (mFufol) as adjuvant treatment for Dukes B2 and C colon cancer patients. Journal of Clinical Oncology 2005,. ASCO Annual Meeting Proceedings. 200523 (Suppl 16): Abstract 3522

789 Andre $T$ et al. Semimonthly versus monthly regimen of fluorouracil and leucovorin administered for 24 or 36 weeks as adjuvant therapy in stage II and III colon cancer: results of a randomized trial. J Clin Oncol 2003; 21 (15): 2896-2903

790 Arkenau HT, Rettig K, Porschen R. Adjuvant chemotherapy in curative resected colon carcinoma: 5-fluorouracil/leucovorin versus high- dose 5-fluorouracil 24-h infusion/leucovorin versus high-dose 5fluorouracil 24-h infusion. Int J Colorectal Dis 2005; 20 (3): 258-261

791 Chau I et al. A randomised comparison between 6 months of bolus fluorouracil/leucovorin and 12 weeks of protracted venous infusion fluorouracil as adjuvant treatment in colorectal cancer. Ann Oncol 2005; 16 (4): $549-557$

792 Poplin EA et al. Phase III Southwest Oncology Group 9415/Intergroup 0153 randomized trial of fluorouracil, leucovorin, and levamisole versus fluorouracil continuous infusion and levamisole for adjuvant treatment of stage III and high-risk stage II colon cancer. J Clin Oncol 2005; 23 (9): (Suppl 5): 1819-1825

793 O'Connell MJ et al. Prospectively randomized trial of postoperative adjuvant chemotherapy in patients with high-risk colon cancer. J Clin Oncol 1998; 16 (1): $295-300$

794 Haller DG et al. Phase III study of fluorouracil, leucovorin, and levamisole in high-risk stage II and III colon cancer: final report of Intergroup 0089. J Clin Oncol 2005; 23 (34): 8671 - 8678

795 Kapiteijn E et al. Preoperative radiotherapy combined with total mesorectal excision for resectable rectal cancer. N Engl J Med 2001; 345 (9): $638-646$

796 Rodel C, Sauer $R$. Neoadjuvant radiotherapy and radiochemotherapy for rectal cancer. Recent Results Cancer Res 2005; 165: 221 - 230

797 Peeters KC et al. The TME trial after a median follow-up of 6 years: increased local control but no survival benefit in irradiated patients with resectable rectal carcinoma. Ann Surg 2007; 246 (5): 693 - 701

798 Folkesson J et al. Swedish Rectal Cancer Trial: long lasting benefits from radiotherapy on survival and local recurrence rate. J Clin Oncol 2005; 23 (24): 5644-5650

799 Baxter NN, Garcia-Aguilar J. Organ preservation for rectal cancer. J Clin Oncol 2007; 25 (8): 1014-1020

800 Rengan $R$ et al. Distal cT2N0 rectal cancer: is there an alternative to abdominoperineal resection? J Clin Oncol 2005; 23 (22): 4905 - 4912

801 Colorectal Cancer Collaborative Group. Adjuvant radiotherapy for rectal cancer: a systematic overview of 8,507 patients from 22 randomised trials. Lancet 2001; 358 (9290): 1291 - 1304

802 Camma C et al. Preoperative radiotherapy for resectable rectal cancer: A meta-analysis. Jama 2000; 284 (8): 1008-1015

803 Frykholm GJ, Glimelius B, Pahlman L. Preoperative or postoperative irradiation in adenocarcinoma of the rectum: final treatment results of a randomized trial and an evaluation of late secondary effects. Dis Colon Rectum 1993; 36 (6): 564-572

804 Sauer $R$ et al. Preoperative versus postoperative chemoradiotherapy for rectal cancer. N Engl J Med 2004; 351 (17): 1731 - 1740

805 Marijnen CA et al. Impact of short-term preoperative radiotherapy on health-related quality of life and sexual functioning in primary rectal cancer: report of a multicenter randomized trial. J Clin Oncol 2005 23 (9): $1847-1858$

806 Peeters KC et al. Late side effects of short-course preoperative radiotherapy combined with total mesorectal excision for rectal cancer: increased bowel dysfunction in irradiated patients - a Dutch colorectal cancer group study. J Clin Oncol 2005; 23 (25): 6199-6206

807 Junginger $T$ et al. Rectal carcinoma: is too much neoadjuvant therapy performed? Proposals for a more selective MRI based indication. Zentralbl Chir 2006; 131 (4): 275-284

808 Smith N, Brown G. Preoperative staging of rectal cancer. Acta Oncol 2008; 47: $20-31$

809 Sebag-Montefiore D, Stephens R, Quirke P et al. for the NCRI colorectal cancer study group and CRO 7 participants Routine short course preop radiotherapy or selective post-op chemoradiotherapy for resectable rectal cancer? Preliminary results of the MRC CR07 randomised trial. Journal of Clinical Oncology 2006, ASCO Annual Meeting Proceedings (Post-Meeting Edition) 200624 (Suppl 18): Abstract 3511

810 Bujko K et al. Sphincter preservation following preoperative radiotherapy for rectal cancer: report of a randomised trial comparing short-term radiotherapy vs. conventionally fractionated radiochemotherapy. Radiother Oncol 2004; 72 (1): 15-24

811 Bujko K et al. Long-term results of a randomized trial comparing preoperative short-course radiotherapy with preoperative conventionally fractionated chemoradiation for rectal cancer. Br J Surg 2006; 93 (10): 1215 - 1223

812 Marijnen CA et al. No downstaging after short-term preoperative radiotherapy in rectal cancer patients. J Clin Oncol 2001; 19 (7): 1976 1984

813 Bosset JF et al. Chemotherapy with preoperative radiotherapy in rectal cancer. N Engl J Med 2006; 355 (11): 1114-1123 
814 Gerard JP et al. Preoperative radiotherapy with or without concurrent fluorouracil and leucovorin in T3-4 rectal cancers: results of FFCD 9203. J Clin Oncol 2006; 24 (28): 4620-4625

815 Rodel C, Sauer R. Integration of novel agents into combined-modality treatment for rectal cancer patients. Strahlenther Onkol 2007; 183 (5): $227-235$

816 Prolongation of the disease-free interval in surgically treated rectal carcinoma. N Engl J Med 1985; 312 (23): 1465-1472

817 Krook JE et al. Effective surgical adjuvant therapy for high-risk rectal carcinoma. N Engl J Med 1991; 324 (11): 709 - 715

818 Merkel S, Mannsmann U, Siassi M et al. The prognostic inhomogeneity in pT3 rectal carcinomas. Int J Colorectal Dis 2001; 16: 298-304

819 Gunderson LL, Sargent DJ, Tepper JE et al. Impact of T and N substage on survival and disease relapse in adjuvant rectal cancer: a pooled analysis. Int J Radiat Oncol Biol Phys 2002; 54: 386 - 96

820 Marijnen CA et al. Radiotherapy does not compensate for positive resection margins in rectal cancer patients: report of a multicenter randomized trial. Int J Radiat Oncol Biol Phys 2003; 55 (5): 1311 - 1320

821 O'Connell MJ et al. Improving adjuvant therapy for rectal cancer by combining protracted-infusion fluorouracil with radiation therapy after curative surgery. N Engl J Med 1994; 331: 502 - 507

822 Smalley SR et al. Phase III trial of fluorouracil-based chemotherapy regimens plus radiotherapy in postoperative adjuvant rectal cancer: GI INT 0144. J Clin Oncol 2006; 24 (22): 3542-3547

823 Tepper JE et al. Adjuvant therapy in rectal cancer: analysis of stage, sex, and local control - final report of intergroup 0114. J Clin Oncol 2002; 20 (7): $1744-1750$

824 Lee JH et al. Randomized trial of postoperative adjuvant therapy in stage II and III rectal cancer to define the optimal sequence of chemotherapy and radiotherapy: a preliminary report. J Clin Oncol 2002; 20 (7): $1751-1758$

825 Kim BITK, Lee J, Lee J et al. Randomized trial of postoperative adjuvant therapy in stage II and III rectal cancer to define the optimal sequence of chemotherapy and radiotherapy: A final report. ASCO Meeting Abstracts 2007; 25: 4050

826 Rodel C, Sauer $R$. Radiotherapy and concurrent radiochemotherapy for rectal cancer. Surg Oncol 2004; 13 (2): 93-101

827 Akasu T et al. Adjuvant chemotherapy with uracil-tegafur for pathological stage III rectal cancer after mesorectal excision with selective lateral pelvic lymphadenectomy: a multicenter randomized controlled trial. Jpn J Clin Oncol 2006; 36 (4): 237 - 244

828 Collette L, Bosset J, den Dulk M et al. Patients with curative resection of cT3-4 rectal cancer after preoperative radiotherapy or radiochemotherapy: does anybody benefit from adjuvant fluorouracil-based chemotherapy? A trial of the European Organisation for Research and Treatment of Cancer Radiation Oncology Group. J Clin Oncol 2007; 25: $4379-4386$

829 Lehnert T, Knaebel H, Duck $M$ et al. Sequential hepatic and pulmonary resections for metastatic colorectal cancer. Br J Surg 1999; 86: 241 243

830 Saito $Y$ et al. Pulmonary metastasectomy for 165 patients with colorectal carcinoma: A prognostic assessment. J Thorac Cardiovasc Surg 2002; 124 (5): $1007-1013$

831 Pfannschmidt J et al. Prognostic factors and survival after complete resection of pulmonary metastases from colorectal carcinoma: experiences in 167 patients. J Thorac Cardiovasc Surg 2003; 126 (3): 732 739

832 Lee WS et al. Pulmonary resection for metastases from colorectal cancer: prognostic factors and survival. Int J Colorectal Dis 2007; 22 (6): $699-704$

833 Pfannschmidt J, Dienemann $H$, Hoffmann $H$. Surgical resection of pulmonary metastases from colorectal cancer: a systematic review of published series. Ann Thorac Surg 2007; 84 (1): $324-338$

834 Poston GJ et al. OncoSurge: a strategy for improving resectability with curative intent in metastatic colorectal cancer. J Clin Oncol 2005; 23 (28): $7125-7134$

835 Nordlinger B et al. Surgical resection of colorectal carcinoma metastases to the liver. A prognostic scoring system to improve case selection, based on 1568 patients. Association Francaise de Chirurgie. Cancer 1996; 77 (7): $1254-1262$

836 Fong $Y$ et al. Liver resection for colorectal metastases. J Clin Oncol 1997; 15 (3): 938 - 946

837 Scheele J et al. Resection of colorectal liver metastases. What prognostic factors determine patient selection? Chirurg 2001; 72 (5): 547 560
838 Kato T et al. Therapeutic results for hepatic metastasis of colorectal cancer with special reference to effectiveness of hepatectomy: analysis of prognostic factors for 763 cases recorded at 18 institutions. Dis Colon Rectum 2003; 46 (Suppl 10): S22-S31

839 Fong Y, Fortner J, Sun RL et al. Clinical score for predicting recurrence after hepatic resection for metastatic colorectal cancer: analysis of 1001 consecutive cases. Ann Surg 1999; 230 (3): 309 - 318; discussion 318-321

840 Schmiegel $W$ et al. Update S3-guideline "colorectal cancer" 2008. Z Gastroenterol 2008; 46 (8): $799-840$

841 Nordlinger $B$ et al. Perioperative chemotherapy with FOLFOX4 and surgery versus surgery alone for resectable liver metastases from colorectal cancer (EORTC Intergroup trial 40983): a randomised controlled trial. Lancet 2008; 371 (9617): 1007-1016

842 Leonard GD, Brenner B, Kemeny NE. Neoadjuvant chemotherapy before liver resection for patients with unresectable liver metastases from colorectal carcinoma. J Clin Oncol 2005; 23 (9): 2038 -2048

843 Lorenz $M$ et al. Prospective pilot study of neoadjuvant chemotherapy with 5-fluorouracil, folinic acid and oxaliplatin in resectable liver metastases of colorectal cancer. Analysis of 42 neoadjuvant chemotherapies. Zentralbl Chir 2003; 128 (2): 87 -94

844 Wein $A$ et al. Neoadjuvant treatment with weekly high-dose 5-Fluorouracil as 24-hour infusion, folinic acid and oxaliplatin in patients with primary resectable liver metastases of colorectal cancer. Oncology 2003; 64 (2): 131 -138

845 Gruenberger $B$ et al. Bevacizumab, capecitabine, and oxaliplatin as neoadjuvant therapy for patients with potentially curable metastatic colorectal cancer. J Clin Oncol 2008; 26 (11): 1830-1835

846 Langer B, Bleiberg H, Labianca $R$ et al. Fluorouracil (FU) plus l-leucovorin (l-LV) versus observation after potentially curative resection of liver or lung metastases from colorectal cancer (CRC): results of the ENG (EORTC/NCIC CTG/GIVIO) randomized trial. Proc Am Soc Clin Oncol 2002; 21 (abstr 592)

847 Mitry E, Fields A, Bleiberg H et al. Adjuvant chemotherapy after potentially curative resection of metastases from colorectal cancer. A metaanalysis of two randomized trials. Journal of Clinical Oncology 2006, ASCO Annual Meeting Proceedings Part I 2006; 24 (Suppl 18): Abstract 3524

848 Portier $G$ et al. Multicenter randomized trial of adjuvant fluorouracil and folinic acid compared with surgery alone after resection of colorectal liver metastases: FFCD ACHBTH AURC 9002 trial. J Clin Oncol 2006; 24 (31): $4976-4982$

849 Parks $R$ et al. Adjuvant chemotherapy improves survival after resection of hepatic colorectal metastases: analysis of data from two continents. J Am Coll Surg 2007; 204 (5): 753 - 761; discussion 761-763

850 Figueras J et al. Resection rate and effect of postoperative chemotherapy on survival after surgery for colorectal liver metastases. Br J Surg 2001; 88 (7): $980-985$

851 Scheithauer W et al. Randomised comparison of combination chemotherapy plus supportive care with supportive care alone in patients with metastatic colorectal cancer. Bmj 1993; 306 (6880): 752 - 755

852 Nordic Gastrointestinal Tumor Adjuvant Therapy Group. Expectancy or primary chemotherapy in patients with advanced asymptomatic colorectal cancer: a randomized trial. J Clin Oncol 1992; 10 (6): 904-911

853 Mattioli $R$ et al. High activity and reduced neurotoxicity of bi-fractionated oxaliplatin plus 5-fluorouracil/leucovorin for elderly patients with advanced colorectal cancer. Ann Oncol 2005; 16 (7): 1147 1151

854 Feliu J et al. XELOX (capecitabine plus oxaliplatin) as first-line treatment for elderly patients over 70 years of age with advanced colorectal cancer. Br J Cancer 2006; 94 (7): 969-975

855 Sastre J et al. Irinotecan in combination with fluorouracil in a 48-hour continuous infusion as first-line chemotherapy for elderly patients with metastatic colorectal cancer: a Spanish Cooperative Group for the Treatment of Digestive Tumors study. J Clin Oncol 2005; 23 (15): $3545-3551$

856 Barrueco J, Marshall J, Mitchell E et al. Safety and efficacy of first-line irinotecan/fluoropymidine combinations in $\mathrm{mCRC}$ patients $>65$ years compared with those <65: The BICC-C study. Journal of Clinical Oncology 2007, ASCO Annual Meeting Proceedings Part I 2007; 25 (Suppl 18): Abstract 4076

857 Folprecht G, Seymour MT, Saltz L et al. Irinotecan/Fluorouracil Combination in First-Line Therapy of Older and Younger Patients With Metastatic Colorectal Cancer: Combined Analysis of 2,691 Patients in 
Randomized Controlled Trials. Journal of Clinical Oncology 2008; 26 (9): $1443-1451$

858 Giacchetti S et al. Long-term survival of patients with unresectable colorectal cancer liver metastases following infusional chemotherapy with 5-fluorouracil, leucovorin, oxaliplatin and surgery. Ann Oncol 1999; 10 (6): $663-669$

859 Falcone A et al. Phase III trial of infusional fluorouracil, leucovorin, oxaliplatin, and irinotecan (FOLFOXIRI) compared with infusional fluorouracil, leucovorin, and irinotecan (FOLFIRI) as first-line treatment for metastatic colorectal cancer: the Gruppo Oncologico Nord Ovest. J Clin Oncol 2007; 25 (13): 1670-1676

860 Van Cutsem E, Nowacki M, Lang I et al. Randomized phase III study of irinotecan and 5-FU/FA with or without cetuximab in the first-line treatment of patients with metastatic colorectal cancer (mCRC): The CRYSTAL trial. Journal of Clinical Oncology 2007,. ASCO Annual Meeting Proceedings Part I 200725 (Suppl 18): Abstract 4000

861 Van Cutsem E, Lang I, D'haens $G$ et al. KRAS status and efficacy in the first-line treatment of patients with metastatic colorectal cancer (mCRC) treated with FOLFIRI with or without cetuximab: The CRYSTAL experience. J Clin Oncol 2008; 26 (abstr 2)

862 Folprecht $G$ et al. Neoadjuvant treatment of unresectable colorectal liver metastases: correlation between tumour response and resection rates. Ann Oncol 2005; 16 (8): 1311 - 1319

863 Souglakos J et al. FOLFOXIRI (folinic acid, 5-fluorouracil, oxaliplatin and irinotecan) vs. FOLFIRI (folinic acid, 5-fluorouracil and irinotecan) as first-line treatment in metastatic colorectal cancer (MCC): a multicentre randomised phase III trial from the Hellenic Oncology Research Group (HORG). Br J Cancer 2006; 94 (6): 798 - 805

864 Saltz LB et al. Bevacizumab in combination with oxaliplatin-based chemotherapy as first-line therapy in metastatic colorectal cancer: a randomized phase III study. J Clin Oncol 2008; 26 (12): 2013-2019

865 Aloia $T$ et al. Liver histology and surgical outcomes after preoperative chemotherapy with fluorouracil plus oxaliplatin in colorectal cancer liver metastases. J Clin Oncol 2006; 24 (31): 4983 - 4990

866 Vauthey JN et al. Chemotherapy regimen predicts steatohepatitis and an increase in 90-day mortality after surgery for hepatic colorectal metastases. J Clin Oncol 2006; 24 (13): 2065-2072

867 Benoist $S$ et al. Complete response of colorectal liver metastases after chemotherapy: does it mean cure? J Clin Oncol 2006; 24 (24): 3939 3945

868 Koopman $M$ et al. Sequential versus combination chemotherapy with capecitabine, irinotecan, and oxaliplatin in advanced colorectal cancer (CAIRO): a phase III randomised controlled trial. Lancet 2007; 370 (9582): $135-142$

869 Schmoll HJ, Sargent D. Single agent fluorouracil for first-line treatment of advanced colorectal cancer as standard? Lancet 2007; 370 (9582): 105-107

870 Seymour MT et al. Different strategies of sequential and combination chemotherapy for patients with poor prognosis advanced colorectal cancer (MRC FOCUS): a randomised controlled trial. Lancet 2007; 370 (9582): $143-152$

871 Dy GK et al. Impact of complete response to chemotherapy on overall survival in advanced colorectal cancer: results from Intergroup N9741. J Clin Oncol 2007; 25 (23): 3469-3474

872 Grothey A et al. Survival of patients with advanced colorectal cancer improves with the availability of fluorouracil-leucovorin, irinotecan, and oxaliplatin in the course of treatment. J Clin Oncol 2004; 22 (7): $1209-1214$

873 Petrelli $N$ et al. The modulation of fluorouracil with leucovorin in metastatic colorectal carcinoma: a prospective randomized phase III trial. Gastrointestinal Tumor Study Group. J Clin Oncol 1989; 7 (10): $1419-1426$

874 Poon MA et al. Biochemical modulation of fluorouracil: evidence of significant improvement of survival and quality of life in patients with advanced colorectal carcinoma. J Clin Oncol 1989; 7 (10): $1407-18$

875 Lokich JJ et al. A prospective randomized comparison of continuous infusion fluorouracil with a conventional bolus schedule in metastatic colorectal carcinoma: a Mid-Atlantic Oncology Program Study. J Clin Oncol 1989; 7 (4): 425-432

876 Gramont $A$ et al. Randomized trial comparing monthly low-dose leucovorin and fluorouracil bolus with bimonthly high-dose leucovorin and fluorouracil bolus plus continuous infusion for advanced colorectal cancer: a French intergroup study. J Clin Oncol 1997; 15 (2): 808 815
877 Efficacy of intravenous continuous infusion of fluorouracil compared with bolus administration in advanced colorectal cancer. J Clin Oncol 1998; 16 (1): $301-308$

878 Clarke DE, Raffin TA. Infectious complications of indwelling long-term central venous catheters. Chest 1990; 97 (4): 966 - 972

879 Prandoni P et al. Treatment of deep venous thrombosis by fixed doses of a low-molecular-weight heparin (CY216). Haemostasis 1990; 20 (Suppl 1): 220-223

880 Verso M, Agnelli $G$. Venous thromboembolism associated with longterm use of central venous catheters in cancer patients. J Clin Oncol 2003; 21 (19): 3665 - 3675

881 Liu G et al. Patient preferences for oral versus intravenous palliative chemotherapy. J Clin Oncol 1997; 15 (1): 110-115

882 Borner MM et al. Patient preference and pharmacokinetics of oral modulated UFT versus intravenous fluorouracil and leucovorin: a randomised crossover trial in advanced colorectal cancer. Eur J Cancer 2002; 38 (3): 349-358

883 Twelves $C$ et al. A randomised cross-over trial comparing patient preference for oral capecitabine and 5-fluorouracil/leucovorin regimens in patients with advanced colorectal cancer. Ann Oncol 2006; 17 (2): $239-245$

884 Van Cutsem E et al. Oral capecitabine compared with intravenous fluorouracil plus leucovorin in patients with metastatic colorectal cancer: results of a large phase III study. J Clin Oncol 2001; 19 (21): 4097-4106

885 Hoff PM et al. Comparison of oral capecitabine versus intravenous fluorouracil plus leucovorin as first-line treatment in 605 patients with metastatic colorectal cancer: results of a randomized phase III study. J Clin Oncol 2001; 19 (8): 2282-2292

886 Van Cutsem $E$ et al. Oral capecitabine vs. intravenous 5-fluorouracil and leucovorin: integrated efficacy data and novel analyses from two large, randomised, phase III trials. Br J Cancer 2004; 90 (6): $1190-1197$

887 Kohne $\mathrm{CH}$ et al. Clinical determinants of survival in patients with 5fluorouracil-based treatment for metastatic colorectal cancer: results of a multivariate analysis of 3825 patients. Ann Oncol 2002; 13 (2): $308-317$

888 Gramont $A$ et al. Leucovorin and fluorouracil with or without oxaliplatin as first-line treatment in advanced colorectal cancer. J Clin Oncol 2000; 18 (16): 2938 - 2947

889 Giacchetti $S$ et al. Phase III trial comparing 4-day chronomodulated therapy versus 2-day conventional delivery of fluorouracil, leucovorin, and oxaliplatin as first-line chemotherapy of metastatic colorectal cancer: the European Organisation for Research and Treatment of Cancer Chronotherapy Group. J Clin Oncol 2006; 24 (22): 3562 - 3569

890 Giacchetti $S$ et al. Phase III multicenter randomized trial of oxaliplatin added to chronomodulated fluorouracil-leucovorin as first-line treatment of metastatic colorectal cancer. J Clin Oncol 2000; 18 (1): 136 147

891 Douillard JY et al. Irinotecan combined with fluorouracil compared with fluorouracil alone as first-line treatment for metastatic colorectal cancer: a multicentre randomised trial. Lancet 2000; 355 (9209): $1041-1047$

892 Kohne $\mathrm{CH}$ et al. Phase III study of weekly high-dose infusional fluorouracil plus folinic acid with or without irinotecan in patients with metastatic colorectal cancer: European Organisation for Research and Treatment of Cancer Gastrointestinal Group Study 40986. J Clin Oncol 2005; 23 (22): $4856-4865$

893 Saltz LB et al. Irinotecan plus fluorouracil and leucovorin for metastatic colorectal cancer. Irinotecan Study Group. N Engl J Med 2000; 343 (13): $905-914$

894 Tournigand $C$ et al. FOLFIRI followed by FOLFOX6 or the reverse sequence in advanced colorectal cancer: a randomized GERCOR study. J Clin Oncol 2004; 22 (2): 229-237

895 Colucci $G$ et al. Phase III randomized trial of FOLFIRI versus FOLFOX4 in the treatment of advanced colorectal cancer: a multicenter study of the Gruppo Oncologico Dell'Italia Meridionale. J Clin Oncol 2005; 23 (22): $4866-4875$

896 Goldberg RM et al. A randomized controlled trial of fluorouracil plus leucovorin, irinotecan, and oxaliplatin combinations in patients with previously untreated metastatic colorectal cancer. J Clin Oncol 2004; 22 (1): $23-30$

897 Goldberg RM et al. Randomized controlled trial of reduced-dose bolus fluorouracil plus leucovorin and irinotecan or infused fluorouracil plus leucovorin and oxaliplatin in patients with previously untreated 
metastatic colorectal cancer: a North American Intergroup Trial. J Clin Oncol 2006; 24 (21): 3347-3353

898 Comella $P$ et al. Oxaliplatin plus high-dose folinic acid and 5-fluorouracil i.v. bolus (OXAFAFU) versus irinotecan plus high-dose folinic acid and 5-fluorouracil i. v. bolus (IRIFAFU) in patients with metastatic colorectal carcinoma: a Southern Italy Cooperative Oncology Group phase III trial. Ann Oncol 2005; 16 (6): 878-886

899 Diaz-Rubio E et al. Phase III study of capecitabine plus oxaliplatin compared with continuous-infusion fluorouracil plus oxaliplatin as first-line therapy in metastatic colorectal cancer: final report of the Spanish Cooperative Group for the Treatment of Digestive Tumors Trial. J Clin Oncol 2007; 25 (27): 4224-4230

900 Porschen $R$ et al. Phase III study of capecitabine plus oxaliplatin compared with fluorouracil and leucovorin plus oxaliplatin in metastatic colorectal cancer: a final report of the AIO Colorectal Study Group. J Clin Oncol 2007; 25 (27): 4217-4223

901 Cassidy J et al. Randomized phase III study of capecitabine plus oxaliplatin compared with fluorouracil/folinic acid plus oxaliplatin as first-line therapy for metastatic colorectal cancer. J Clin Oncol 2008; 26 (12): 2006-2012

902 Fuchs CS et al. Randomized, controlled trial of irinotecan plus infusional, bolus, or oral fluoropyrimidines in first-line treatment of metastatic colorectal cancer: results from the BICC-C Study. J Clin Oncol 2007; 25 (30): 4779-4786

903 Kohne $\mathrm{CH}$ et al. Irinotecan combined with infusional 5-fluorouracil/ folinic acid or capecitabine plus celecoxib or placebo in the first-line treatment of patients with metastatic colorectal cancer. EORTC study 40015. Ann Oncol 2008; 19 (5): 920-926

904 Schmiegel WH, Reinacher-Schick A, Freier W et al. Comparable safety and response rate with bevacizumab in combination with capecitabine/oxaliplatin (CapOx/Bev) versus capecitabine/irinotecan (CapIri/ $\mathrm{Bev}$ ) in advanced CRC (mCRC): A randomized phase II study of the AIO GI tumor study group. Journal of Clinical Oncology 2007, ASCO Annual Meeting Proceedings Part I 200725 (Suppl 18): 4034

905 Kabbinavar FF et al. Addition of bevacizumab to bolus fluorouracil and leucovorin in first-line metastatic colorectal cancer: results of a randomized phase II trial. J Clin Oncol 2005; 23 (16): 3697-3705

906 Hurwitz H et al. Bevacizumab plus irinotecan, fluorouracil, and leucovorin for metastatic colorectal cancer. N Engl J Med 2004; 350 (23): $2335-2342$

907 Bokemeyer C, Bondarenko I, Makhson A et al. Cetuximab plus 5-FU/FA/ oxaliplatin (FOLFOX-4) versus FOLFOX-4 in the first-line treatment of metastatic colorectal cancer (mCRC): OPUS, a randomized phase II study. Journal of Clinical Oncology 2007, ASCO Annual Meeting Proceedings Part I 200725 (Suppl 18): 4035

908 Folprecht $G$ et al. Cetuximab and irinotecan/5-fluorouracil/folinic acid is a safe combination for the first-line treatment of patients with epidermal growth factor receptor expressing metastatic colorectal carcinoma. Ann Oncol 2006; 17 (3): 450-456

909 Tournigand $C$ et al. OPTIMOX1: a randomized study of FOLFOX4 or FOLFOX7 with oxaliplatin in a stop-and-Go fashion in advanced colorectal cancer - a GERCOR study. J Clin Oncol 2006; 24 (3): 394-400

910 Maindrault-Goebel F, Lledo G, Chibaudel B et al. Final results of OPTIMOX2, a large randomized phase II study of maintenance therapy or chemotherapy-free intervals (CFI) after FOLFOX in patients with metastatic colorectal cancer (MRC): A GERCOR study. Journal of Clinical Oncology 2007, ASCO Annual Meeting Proceedings Part I 200725 (Suppl 18): 4013

911 Maughan TS et al. Comparison of intermittent and continuous palliative chemotherapy for advanced colorectal cancer: a multicentre randomised trial. Lancet 2003; 361 (9356): $457-464$

912 Labianca R et al. Alternating versus continuous "FOLFIRI" in advanced colorectal cancer (ACC): A randomized "GISCAD" trial. Journal of Clinical Oncology 2006, ASCO Annual Meeting Proceedings Part I 2006; 24 (Suppl 18)

913 Cunningham $D$ et al. Randomised trial of irinotecan plus supportive care versus supportive care alone after fluorouracil failure for patients with metastatic colorectal cancer. Lancet 1998; 352 (9138): $1413-1418$

914 Rougier $P$ et al. Randomised trial of irinotecan versus fluorouracil by continuous infusion after fluorouracil failure in patients with metastatic colorectal cancer. Lancet 1998; 352 (9138): 1407-1412

915 Rothenberg ML et al. Superiority of oxaliplatin and fluorouracil-leucovorin compared with either therapy alone in patients with progressive colorectal cancer after irinotecan and fluorouracil-leucovorin: in- terim results of a phase III trial. J Clin Oncol 2003; 21 (11): 20592069

916 Cunningham D et al. Cetuximab monotherapy and cetuximab plus irinotecan in irinotecan-refractory metastatic colorectal cancer. $\mathrm{N}$ Engl J Med 2004; 351 (4): 337-345

917 Sobrero AF et al. EPIC: phase III trial of cetuximab plus irinotecan after fluoropyrimidine and oxaliplatin failure in patients with metastatic colorectal cancer. J Clin Oncol 2008; 26 (14): 2311-2319

918 Giantonio BJ et al. Bevacizumab in combination with oxaliplatin, fluorouracil, and leucovorin (FOLFOX4) for previously treated metastatic colorectal cancer: results from the Eastern Cooperative Oncology Group Study E3200. J Clin Oncol 2007; 25 (12): 1539-1544

919 Rothenberg ML et al. Phase III trial of capecitabine + oxaliplatin (XELOX) vs. 5-fluorouracil (5-FU), leucovorin (LV), and oxaliplatin (FOLFOX4) as 2nd-line treatment for patients with metastatic colorectal cancer (MCRC). Journal of Clinical Oncology 2007, ASCO Annual Meeting Proceedings (Post-Meeting Edition) 200725 (Suppl 18):

920 Eng C, Maurel J, Scheithauer W et al. Impact on quality of life of adding cetuximab to irinotecan in patients who have failed prior oxaliplatinbased therapy: the EPIC trial. Proc Am Soc Clin Oncol 2007; 25: 164s Abstract 4003

921 Rao S et al. Phase II study of capecitabine and mitomycin C as first-line treatment in patients with advanced colorectal cancer. Br J Cancer 2004; 91 (5): 839-843

922 Chong $G$ et al. Capecitabine and mitomycin $C$ as third-line therapy for patients with metastatic colorectal cancer resistant to fluorouracil and irinotecan. Br J Cancer 2005; 93 (5): 510 - 514

$923 \mathrm{Lim}$ DH et al. Mitomycin-C and capecitabine as third-line chemotherapy in patients with advanced colorectal cancer: a phase II study. Cancer Chemother Pharmacol 2005; 56 (1): 10 - 14

924 Alliot $C$. Capecitabine and mitomycin $C$ in patients with metastatic colorectal cancer resistant to fluorouracil and irinotecan. Br J Cancer 2006; 94 (6): 935 - 936; author reply 937

925 Lenz HJ et al. Multicenter phase II and translational study of cetuximab in metastatic colorectal carcinoma refractory to irinotecan, oxaliplatin, and fluoropyrimidines. J Clin Oncol 2006; 24 (30): 49144921

926 Jonker DJ, O'Callaghan CJ, Karapetis CS et al. Cetuximab for the Treatment of Colorectal Cancer. N Engl J Med 2007; 357: 2040 - 2048

927 Lievre A et al. KRAS mutations as an independent prognostic factor in patients with advanced colorectal cancer treated with cetuximab. J Clin Oncol 2008; 26 (3): 374-379

928 Hecht JR et al. Panitumumab monotherapy in patients with previously treated metastatic colorectal cancer. Cancer 2007; 110 (5): 980-988

929 Van Cutsem E et al. Open-label phase III trial of panitumumab plus best supportive care compared with best supportive care alone in patients with chemotherapy-refractory metastatic colorectal cancer. J Clin Oncol 2007; 25 (13): 1658-1664

930 Amado RG, Wolf M, Peeters $M$ et al. Wild-Type KRAS Is Required for Panitumumab Efficacy in Patients With Metastatic Colorectal Cancer. Journal of Clinical Oncology 2008; 26 (10): 1626-1634

931 Lehnert T, Golling M. Posterior pelvic exenteration in locoregional recurrence of rectal carcinoma - indications, technique and outcome. Chirurg 2001; 72 (12): 1393-1401

932 Shibata $D$ et al. Surgical management of isolated retroperitoneal recurrences of colorectal carcinoma. Dis Colon Rectum 2002; 45 (6): $795-801$

$933 \mathrm{Wu}$ JS et al. Meta-analysis of dose-fractionation radiotherapy trials for the palliation of painful bone metastases. Int J Radiat Oncol Biol Phys 2003; 55 (3): 594-605

934 Farnell GF et al. Brain metastases from colorectal carcinoma. The long term survivors. Cancer 1996; 78 (4): 711-716

935 Schoeggl $A$ et al. Stereotactic radiosurgery for brain metastases from colorectal cancer. Int J Colorectal Dis 2002; 17 (3): 150 - 155

936 Enblad $P$ et al. The risk of subsequent primary malignant diseases after cancers of the colon and rectum. A nationwide cohort study. Cancer 1990; 65 (9): $2091-2100$

937 Cali RL et al. Cumulative incidence of metachronous colorectal cancer. Dis Colon Rectum 1993; 36 (4): 388-393

938 Hermanek PJr et al. Long-term results of surgical therapy of colon cancer. Results of the Colorectal Cancer Study Group. Chirurg 1994; 65 (4): 287-297

939 Yamazaki $T$ et al. What is the risk factor for metachronous colorectal carcinoma? Dis Colon Rectum 1997; 40 (8): 935 - 938 
940 Hemminki K, Li X, Dong C. Second primary cancers after sporadic and familial colorectal cancer. Cancer Epidemiol Biomarkers Prev 2001; 10 (7): $793-798$

941 Green RJ et al. Surveillance for second primary colorectal cancer after adjuvant chemotherapy: an analysis of Intergroup 0089. Ann Intern Med 2002; 136 (4): 261 - 269

942 Harris GJ et al. Factors affecting local recurrence of colonic adenocarcinoma. Dis Colon Rectum 2002; 45 (8): 1029-1034

943 Merkel S et al. Late locoregional recurrence in rectal carcinoma. Eur J Surg Oncol 2002; 28 (7): 716-722

944 Poulin EC et al. Local recurrence and survival after laparoscopic mesorectal resection forrectal adenocarcinoma. Surg Endosc 2002; 16 (6): 989-995

945 Bulow $S$ et al. Recurrence and survival after mesorectal excision for rectal cancer. Br J Surg 2003; 90 (8): 974-980

946 Secco GB et al. Factors influencing incidence and extension of metachronous liver metastases of colorectal adenocarcinoma. A multivariate analysis. Hepatogastroenterology 1997; 44 (16): 1057-1062

947 Pietra $N$ et al. Risk factors of local recurrence of colorectal cancer: a multivariate study. Hepatogastroenterology 1998; 45 (23): 15731578

948 Scholmerich J. Sense and nonsense in after-care of colorectal carcinoma. Med Klin (Munich) 1996; 91 (6): 420-427

949 Kievit J. Colorectal cancer follow-up: a reassessment of empirical evidence on effectiveness. Eur J Surg Oncol 2000; 26 (4): 322 - 328

950 Kievit J. Follow-up of patients with colorectal cancer: numbers needed to test and treat. Eur J Cancer 2002; 38 (7): 986-999

951 Wichmann MW et al. Results of long-term follow-up after curative resection of Dukes A colorectal cancer. World J Surg 2002; 26 (6): 732 736

952 Yamamoto $S$ et al. Postsurgical surveillance for recurrence of UICC stage I colorectal carcinoma: is follow-up by CEA justified? Hepatogastroenterology 2005; 52 (62): 444-449

953 Korner $\mathrm{H}$ et al. Systematic follow-up after curative surgery for colorectal cancer in Norway: a population-based audit of effectiveness, costs, and compliance. J Gastrointest Surg 2005; 9 (3): 320-328

954 Talbot IC et al. The clinical significance of invasion of veins by rectal cancer. Br J Surg 1980; 67 (6): 439-442

955 Minsky BD et al. Resectable adenocarcinoma of the rectosigmoid and rectum. II. The influence of blood vessel invasion. Cancer 1988; 61 (7): $1417-1424$

956 Krasna MJ et al. Vascular and neural invasion in colorectal carcinoma. Incidence and prognostic significance. Cancer 1988; 61 (5): 1018 1023

957 Inoue $T$ et al. Vascular invasion of colorectal carcinoma readily visible with certain stains. Dis Colon Rectum 1992; 35 (1): $34-39$

958 Bruinvels DJ et al. Follow-up of patients with colorectal cancer. A meta-analysis. Ann Surg 1994; 219 (2): 174-182

959 Rosen $M$ et al. Follow-up of colorectal cancer: a meta-analysis. Dis Colon Rectum 1998; 41 (9): 1116-1126

960 Jeffery GM, Hickey BE, Hider P. Follow-up strategies for patients treated for non-metastatic colorectal cancer. Cochrane Database Syst Rev 2002; 1: CD002200

961 Renehan AG et al. Impact on survival of intensive follow up after curative resection for colorectal cancer: systematic review and meta-analysis of randomised trials. Bmj 2002; 324 (7341): 813

962 Makela JT, Laitinen SO, Kairaluoma MI. Five-year follow-up after radical surgery for colorectal cancer. Results of a prospective randomized trial. Arch Surg 1995; 130 (10): $1062-1067$

963 Ohlsson B et al. Follow-up after curative surgery for colorectal carcinoma. Randomized comparison with no follow-up. Dis Colon Rectum 1995: 38 (6): 619-626

964 Kjeldsen BJ et al. A prospective randomized study of follow-up after radical surgery for colorectal cancer. Br J Surg 1997; 84 (5): 666-669

965 Pietra $N$ et al. Role of follow-up in management of local recurrences of colorectal cancer: a prospective, randomized study. Dis Colon Rectum 1998; 41 (9): 1127-1133

966 Schoemaker D et al. Yearly colonoscopy, liver CT, and chest radiography do not influence 5-year survival of colorectal cancer patients. Gastroenterology 1998; 114 (1): 7-14

967 Secco GB et al. Efficacy and cost of risk-adapted follow-up in patients after colorectal cancer surgery: a prospective, randomized and controlled trial. Eur J Surg Oncol 2002; 28 (4): 418 - 423
968 Jeffery M, Hickey BE, Hider PN. Follow-up strategies for patients treated for non-metastatic colorectal cancer. Cochrane Database Syst Rev 2007; 1: CD002200

969 Wattchow DA et al. General practice vs. surgical-based follow-up for patients with colon cancer: randomised controlled trial. Br J Cancer 2006; 94 (8): 1116-1121

970 Rodriguez-Moranta F et al. Postoperative surveillance in patients with colorectal cancer who have undergone curative resection: a prospective, multicenter, randomized, controlled trial. J Clin Oncol 2006; 24 (3): $386-393$

971 Laubert $T$ et al. Intensified surveillance after surgery for colorectal cancer significantly improves survival. Eur J Med Res 2010; 15 (1) $25-30$

972 Papagrigoriadis S, Heyman B. Patients' views on follow up of colorectal cancer: implications for risk communication and decision making. Postgrad Med J 2003; 79 (933): $403-407$

973 Cooper GS, TD Kou, HL ReynoldsJr. Receipt of guideline-recommended follow-up in older colorectal cancer survivors: a population-based analysis. Cancer 2008; 113 (8): 2029-2037

974 Rocklin MS, Slomski CA, Watne AL. Postoperative surveillance of patients with carcinoma of the colon and rectum. Am Surg 1990; 56 (1): $22-27$

975 Benson AB 3rd et al. 2000 update of American Society of Clinical Oncology colorectal cancer surveillance guidelines. J Clin Oncol 2000; 18 (20): $3586-3588$

976 Berman JM, Cheung RJ, Weinberg DS. Surveillance after colorectal cancer resection. Lancet 2000; 355 (9201): 395 - 399

977 Desch CE et al. Recommended colorectal cancer surveillance guidelines by the American Society of Clinical Oncology. J Clin Oncol 1999; 17 (4): 1312

978 Macdonald JS. Carcinoembryonic antigen screening: pros and cons. Semin Oncol 1999; 26 (5): 556-560

979 Duffy MJ et al. Clinical utility of biochemical markers in colorectal cancer: European Group on Tumour Markers (EGTM) guidelines. Eur J Cancer 2003; 39 (6): 718 - 727

980 Duffy MJ. Carcinoembryonic antigen as a marker for colorectal cancer: is it clinically useful? Clin Chem 2001; 47 (4): 624-630

981 Moertel CG et al. An evaluation of the carcinoembryonic antigen (CEA) test for monitoring patients with resected colon cancer. Jama 1993; $270(8): 943-947$

982 Safi F, Beyer HG. The value of follow-up after curative surgery of colorectal carcinoma. Cancer Detect Prev 1993; 17 (3): 417-424

983 Zeng Z, Cohen AM, Urmacher C. Usefulness of carcinoembryonic antigen monitoring despite normal preoperative values in node-positive colon cancer patients. Dis Colon Rectum 1993; 36 (11): 1063-1068

984 Huebner $R H$ et al. A meta-analysis of the literature for whole-body FDG PET detection of recurrent colorectal cancer. J Nucl Med 2000; 41 (7): $1177-1189$

985 Annovazzi A et al. 18-fluorodeoxyglucose positron emission tomography in nonendocrine neoplastic disorders of the gastrointestinal tract. Gastroenterology 2003; 125 (4): 1235 - 1245

986 Ahlquist DA et al. Accuracy of fecal occult blood screening for colorectal neoplasia. A prospective study using Hemoccult and HemoQuant tests. Jama 1993; 269 (10): 1262-1267

987 Hunerbein $M$ et al. The role of transrectal ultrasound-guided biopsy in the postoperative follow-up of patients with rectal cancer. Surgery 2001; 129 (2): $164-169$

988 Mitry E et al. Epidemiology, management and prognosis of colorectal cancer with lung metastases: a 30-year population-based study. Gut 2010; 59 (10): $1383-1388$

989 Desch CE et al. Colorectal cancer surveillance: 2005 update of an American Society of Clinical Oncology practice guideline. J Clin Oncol 2005; 23 (33): $8512-8519$

990 Chau I et al. The value of routine serum carcino-embryonic antigen measurement and computed tomography in the surveillance of patients after adjuvant chemotherapy for colorectal cancer. J Clin Oncol 2004; 22 (8): 1420-1429

991 Sugarbaker PH et al. A simplified plan for follow-up of patients with colon and rectal cancer supported by prospective studies of laboratory and radiologic test results. Surgery 1987; $102(1)$ : 79-87

992 Wang $T$ et al. The role of postoperative colonoscopic surveillance after radical surgery for colorectal cancer: a prospective, randomized clinical study. Gastrointest Endosc 2009; 69 (3): 609-615 
993 Rulyak SJ et al. Outcome of follow-up colon examination among a population-based cohort of colorectal cancer patients. Clin Gastroenterol Hepatol 2007; 5 (4): 470 - 476; quiz 407

994 Rex DK et al. Guidelines for colonoscopy surveillance after cancer resection: a consensus update by the American Cancer Society and the US Multi-Society Task Force on Colorectal Cancer. Gastroenterology 2006; 130 (6): $1865-1871$

995 Bouvier AM et al. The lifelong risk of metachronous colorectal cancer justifies long-term colonoscopic follow-up. Eur J Cancer 2008; 44 (4): $522-527$

996 Sobhani I et al. Early detection of recurrence by 18FDG-PET in the follow-up of patients with colorectal cancer. Br J Cancer 2008; 98 (5): $875-880$

997 Selvaggi $\mathrm{F}$ et al. FGD-PET in the follow-up of recurrent colorectal cancer. Colorectal Dis 2003; 5 (5): 496 - 500

998 Eckardt VF, Bernhard G. Nachsorge beim kolorektalen Karzinom. Eine Analyse von Effektivität und Kosten. Deutsches Ärzteblatt 1997; 94: A456-A462

999 Schwibbe G. Changes in quality of life in oncological patients in the course of an inpatient after-care program. Rehabilitation (Stuttg) 1991; 30 (2): 55-62

1000 Gartner U et al. Physical complaints, stress and quality of life of oncologic patients. Effects and patient assessment in inpatient rehabilitation. Med Klin (Munich) 1996; 91 (8): 501 -508

1001 Etzioni DA, El-Khoueiry AB, Beart RW Jr. Rates and predictors of chemotherapy use for stage III colon cancer: a systematic review. Cancer 2008; 113 (12): 3279-3289

1002 Grothey A, Kellermann L, Schmoll HJ. Deficits in management of patients with colorectal carcinoma in Germany. Results of multicenter documentation of therapy algorithms. Med Klin (Munich) 2002; 97 (5): $270-277$

1003 von der Schulenburg JM, Prenzler A, Schurer W. Cancer management and reimbursement aspects in Germany: an overview demonstrated by the case of colorectal cancer. Eur J Health Econ 2010; 10 (Suppl 1): S21 -S26

1004 Adler G, Seufferlein T. Hope and disappointment in cancer medicine. Z Arztl Fortbild Qualitatssich 2007; 101 (5): 287-292

1005 Harrison JD et al. What are the unmet supportive care needs of people with cancer? A systematic review. Support Care Cancer 2009; $17: 1117-1128$

1006 Lewis $R$ et al. Nurse-led vs. conventional physician-led follow-up for patients with cancer: systematic review. J Adv Nurs 2009; 65 (4): $706-723$

1007 Howell DM et al. A mixed-method evaluation of nurse-led community-based supportive cancer care. Support Care Cancer 2008; 16 (12): $1343-1352$

1008 Ouwens $M$ et al. Implementation of integrated care for patients with cancer: a systematic review of interventions and effects. Int J Qual Health Care 2009; 21 (2): 137-144

1009 Jahn P et al. Reduction of chemotherapy-induced anorexia, nausea, and emesis through a structured nursing intervention: a clusterrandomized multicenter trial. Support Care Cancer 2009; 17 (12): $1543-1552$
1010 Jahn P et al. Improvement of pain related self management for oncologic patients through a trans institutional modular nursing intervention: protocol of a cluster randomized multicenter trial. Trials 2010; $11: 29$

1011 Chen RC et al. Patient-reported acute gastrointestinal symptoms during concurrent chemoradiation treatment for rectal cancer. Cancer 2010; 116 (8): 1879-1886

1012 Meyerhardt JA et al. Impact of body mass index and weight change after treatment on cancer recurrence and survival in patients with stage III colon cancer: findings from Cancer and Leukemia Group B 89803. J Clin Oncol 2008; 26 (25): 4109-4115

1013 Meyerhardt JA et al. Physical activity and survival after colorectal cancer diagnosis. J Clin Oncol 2006; 24 (22): 3527-3534

1014 Meyerhardt JA et al. Impact of physical activity on cancer recurrence and survival in patients with stage III colon cancer: findings from CALGB 89803. J Clin Oncol 2006; 24 (22): 3535-3541

$1015 \mathrm{Ng}$ K et al. Multivitamin use is not associated with cancer recurrence or survival in patients with stage III colon cancer: findings from CALGB 89803. J Clin Oncol 2010; 28 (28): 4354-4363

1016 Stumpf C et al. Retrospektive Untersuchung zur Therapie mit Mistelextrakten bei Patienten mit kolorektalem Karzinom. Dtsch Zschr Onkol 2007; 39: $12-22$

1017 Friedel $W$ et al. Fermented Europaen mistletoe (Viscum album L.) extract in supportive care in patients with primary non-metastatic colorectal cancer. In: Scheer R, et al., (eds.) Die Mistel in der Tumortherapie Essen: KVC Verlag; 2009: 563-576

1018 Kienle GS, Kiene H. Review article: Influence of Viscum album L (European mistletoe) extracts on quality of life in cancer patients: a systematic review of controlled clinical studies. Integr Cancer Ther 2010; 9 (2): $142-157$

1019 Shimizu $M$ et al. (-)-Epigallocatechin gallate suppresses azoxymethane-induced colonic premalignant lesions in male $\mathrm{C} 57 \mathrm{BL} / \mathrm{KsJ}-\mathrm{db} /$ db mice. Cancer Prev Res (Phila) 2008; 1 (4): 298 - 304

1020 Hoensch $\mathrm{H}$ et al. Prospective cohort comparison of flavonoid treatment in patients with resected colorectal cancer to prevent recurrence. World J Gastroenterol 2008; 14 (14): 2187-2193

1021 Ito $\mathrm{K}$ et al. Long-term effect of 5 -fluorouracil enhanced by intermittent administration of polysaccharide $\mathrm{K}$ after curative resection of colon cancer. A randomized controlled trial for 7-year follow-up. Int J Colorectal Dis 2004; 19 (2): 157-164

1022 Sakamoto $\mathrm{J}$ et al. Efficacy of adjuvant immunochemotherapy with polysaccharide $\mathrm{K}$ for patients with curatively resected colorectal cancer: a meta-analysis of centrally randomized controlled clinical trials. Cancer Immunol Immunother 2006; 55 (4): 404-411

$1023 \mathrm{Kassab} S$ et al. Homeopathic medicines for adverse effects of cancer treatments. Cochrane Database Syst Rev 2009; 2: CD004845

1024 Rahbari NN et al. Definition and grading of anastomotic leakage following anterior resection of the rectum: a proposal by the International Study Group of Rectal Cancer. Surgery 2010; 147 (3): 339351

1025 Vasen HF et al. New clinical criteria for hereditary nonpolyposis colorectal cancer (HNPCC, Lynch syndrome) proposed by the International Collaborative group on HNPCC. Gastroenterology 1999; 116 (6): $1453-1456$ 